\title{
Ultrasonic duplex scanning in atherosclerotic disease of the vertebrobasilar arterial system : a non-invasive technique compared with contrast arteriography
}

Citation for published version (APA):

Ackerstaff, R. G. A. (1985). Ultrasonic duplex scanning in atherosclerotic disease of the vertebrobasilar arterial system : a non-invasive technique compared with contrast arteriography. [Doctoral Thesis, Maastricht University]. Rijksuniversiteit Limburg. https://doi.org/10.26481/dis.19850530ra

Document status and date:

Published: 01/01/1985

DOI:

10.26481/dis.19850530ra

Document Version:

Publisher's PDF, also known as Version of record

Please check the document version of this publication:

- A submitted manuscript is the version of the article upon submission and before peer-review. There can be important differences between the submitted version and the official published version of record.

People interested in the research are advised to contact the author for the final version of the publication, or visit the DOI to the publisher's website.

- The final author version and the galley proof are versions of the publication after peer review.

- The final published version features the final layout of the paper including the volume, issue and page numbers.

Link to publication

\footnotetext{
General rights rights.

- You may freely distribute the URL identifying the publication in the public portal. please follow below link for the End User Agreement:

www.umlib.nl/taverne-license

Take down policy

If you believe that this document breaches copyright please contact us at:

repository@maastrichtuniversity.nl

providing details and we will investigate your claim.
}

Copyright and moral rights for the publications made accessible in the public portal are retained by the authors and/or other copyright owners and it is a condition of accessing publications that users recognise and abide by the legal requirements associated with these

- Users may download and print one copy of any publication from the public portal for the purpose of private study or research.

- You may not further distribute the material or use it for any profit-making activity or commercial gain

If the publication is distributed under the terms of Article 25fa of the Dutch Copyright Act, indicated by the "Taverne" license above, 


\section{Ackerstaft, Robert Gertjan Adrien}

Ultrasonic duplex scanning in atherosclerotic disease of the vertebrobasilar arterial system: a non-invasive technique compared with contrast arteriography / Robert Gertjan Adrien Ackerstaff. - [S.1.: s.n.] (Utrecht :

Elinkwijk). - III.

Proefschrift Rijksuniversiteit Limburg. - Met lit. opg. Met samenvatting in het Nederlands, Frans, Duits, Indonesisch en Japans.

ISBN 90-9000908-6

SISO 605.12 UDC 616.1:612.82

Trefw.: atherosclerose / vertebrobasilaire insufficiëntie ; duplexondlerzoek.

Any part of this book may be reproduced in any form, by print, photoprint, microfilm or by any other means without permission from the author. 


\section{ULTRASONIC DUPLEX SCANNING IN ATHEROSCLEROTIC DISEASE OF THE VERTEBROBASILAR ARTERIAL SYSTEM}

\section{A NON-INVASIVE TECHNIQUE COMPARED WITH CONTRAST ARTERIOGRAPHY}

\section{PROEFSCHRIFT}

ter verkrijging van de graad van doctor in de geneeskunde aan de Rijksuniversiteit Limburg te Maastricht, op gezag van de Rector Magnificus, Prof.Dr. F.I.M. Bonke, volgens het besluit van het College van Dekanen, in het openbaar te verdedigen op donderdag 30 mei 1985 , om 16.00 uur

door

ROBERT GERTJAN ADRIEN ACKERSTAFF geboren te Soerabaja, Indonesië in 1942 
Promotores: Prof.Dr. J.M.F.A. Mol

Prof.Dr. R.S. Reneman

Referenten: Prof.Dr. M. Hennerici

Dr. P.J. Breslau

Financial support by the Netherlands Heart Foundation for the publication of this thesis is gratefully acknowledged (Grant-in-aid 84.138).

The publication of this study was also financially supported by The St. Antonius Hospital, Nieuwegein, The Netherlands; Squibb Medical Systems, Munich, West Germany; and Het Henriëtte Ackerstaff-Korpel Fonds. 


\section{CONTENTS}

1. INTRODUCTION AND PURPOSE OF THE STUDY

\section{PART 1. LITERATURE STUDY}

2. NORMAL ANATOMY, ANATOMIC ANOMALIES, AND COLLATERAL PATHWAYS

2.1. Introduction 15

2.2. Vascular anatomy 15

2.3. Collateral pathways 24

3. PHYSIOLOGY AND PATHOPHYSIOLOGY OF CEREBRAL CIRCULATION

3.1. Introduction $\quad 26$

3.2. Regulation of cerebral circulation 26

3.3. Pathogenesis of neurological deficits 28

4. THE ROLE OF VERTEBRAL ARTERY DISEASE IN THE PATHOGENESIS OF CEREBRAL ISCHAEMIA

4.1. Introduction

4.2. Atherosclerosis of the innominate, subclavian and vertebral arteries

4.3. The significance of atherosclerotic disease of the innominatesubclavian-vertebral system and its interaction with disease of the carotid system

5. SOME SYNDROMES OF VERTEBROBASILAR ARTERIAL INSUFFICIENCY

5.1. Introduction

5.2. Transient ischaemic attacks in the vertebrobasilar territory

5.3. The significance of degenerative osteo-arthritic change of the cervical vertebrae in vertebrobasilar arterial insufficiency

5.4. The subclavian steal syndrome

6. DIAGNOSTIC APPROACHES 54

6.1. Arteriography 54

6.1.1. Complications of vertebral arteriography $\quad 55$

6.1.2. Accuracy of cerebral arteriography $\quad 57$

6.2. Non-invasive tests 60

6.2.1. Non-imaging Doppler techniques 61

6.2.2. Imaging Doppler techniques 63

6.2.3. The duplex concept 64 
7. EQUIPMENT, INSTRUMENTS, AND DATA ANALYSIS OF THE DOPPLER SIGNALS

7.1. The instrumentation $\quad 69$

$\begin{array}{ll}\text { 7.2. Data processing } & 72\end{array}$

8. ULTRASONIC DUPLEX SCANNING OF THE ARCH VESSELS AND VERTEBRAL ARTERIES

$\begin{array}{ll}\text { 8.1. Patient examination method } & 74\end{array}$

8.1.1. The carotid system 77

8.1.2. The innominate artery 77

8.1.3. The subclavian arteries 79

8.1.4. The vertebral arteries $\quad 80$

8.2. Criteria and categories used for diagnosis 83

9. THE ACCURACY OF ULTRASONIC DUPLEX SCANNING OF THE INNOMINATE, SUBCLAVIAN, AND VERTEBRAL ARTERIES, AS COMPARED WITH CONTRAST ARTERIOGRAPHY

9.1. Introduction

9.2. Arteriographic examinations, their numerical description and statistical analysis used

9.3. The first study (May 1981-December 1982) 92

9.3.1. The subclavian artery 93

9.3.2. The vertebral artery 94

9.3.3. Discussion of the results of the first study 95

9.4. The second study (December 1982-October 1984) 97

9.4.1. The innominate artery 98

9.4 .2 . The subclavian artery $\quad 99$

9.4.3. The prevertebral segment of the vertebral artery 101

9.4 .4 . The cervical segment of the vertebral artery 103

9.4.5. The atlantic segment of the vertebral artery $\quad 110$

9.4.6. The significance of ultrasonic duplex scanning in cases of severe, obstructive disease of the intradural segment of the vertebral artery

9.4.7. The subclavian and innominate steal syndromes $\quad 113$

9.4.8. Discussion of the results of the second study 115

10. GENERAL DISCUSSION, RECOMMENDATIONS AND CONCLUSIONS

10.1. Introduction

10.2. Recent developments in diagnosis of atherosclerotic disease of the cerebrovascullar tree 
10.3. The impact of recently developed diagnostic procedures in the evaluation of cerebral atherosclerotic disease on the management of patients

10.4. Ultrasonic duplex scanning of the innominate, subclavian, and vertebral arteries

10.5. Final conclusions and recommendations 145

11. SUMMARIES 148

11.1. English 148

11.2. Dutch 152

11.3. French 156

11.4. German 160

11.5. Indonesian 164

11.6. Japanese 168

$\begin{array}{ll}\text { REFERENCES } & 174\end{array}$

$\begin{array}{ll}\text { ACKNOWLEDGEMENTS } & 195\end{array}$

$\begin{array}{ll}\text { CURRICULUM VITAE } & 196\end{array}$ 



\section{INTRODUCTION AND PURPOSE OF THE STUDY}

Cerebral arterial disease is one of the most perplexing subjects a physician can study. This is not because of the vagaries of vascular disease but because of the complexity of the brain and the selective vulnerability of its many parts to ischaemia. Diseases of the cerebral arterial system usually occur late in life against a background of widespread long-standing vascular changes in many other organs of the body. Often the function of the heart, kidney and limb vessels is similarly impaired by disease and hypertension is an added feature in many cases. Atheromatous cerebral arterial disease, even more than coronary artery disease is but one facet of a generalized disorder. Cerebral infarction rarely has a single cause. Usually it is the result of both obstruction in extracranial or intracranial cerebral arteries and systemic disease. During the last decades many studies appear to have shown that local intracranial factors are less important than extracranial factors in the production of transient and persistent cerebral deficits. As a result of these investigations there is a large body of information concerning the pathological effects of atherosclerotic lesions of the carotid arteries on the brain. Because this part of the cerebrovascular system is easily accessible to diagnostic techniques and surgical procedures, there is an increasing interest about the detection and treatment of its pathology.

When reviewing the literature one may forget that atherosclerosis is a diffuse process, and that the human brain obtains its vascular supply by four main arteries: the carotids and the vertebrals. On the other hand anatomical, pathological, and clinical studies have delineated the importance of collateral circulation between the carotid and the vertebrobasilar territories. An anomalous circle of Willis with a hypoplastic posterior communicating artery for example, is particularly associated with increased vulnerability to stroke (Berry, 1961, Alpers et al., 1963). Involvement of the vertebrobasillar arterial system by the atherosclerotic process increases the stroke risk of patients with obstructive lesions of the carotid arteries (Hennerici et al., 1982). Similarly, because of the interdependence of the carotid and vertebrobasilar systems and the variability of the origin of the dominant blood supply to the posterior cerebral arteries, carotid obstructive disease may present itself with the clinical picture of vertebrobasilar ischaemia and vice versa (Haferkamp et al., 1974, Ueda et al., 1979, Bogousslavsky et al., 1983, 1984, 1985).

Many symptoms of cerebrovascular insufficiency are in fact due to extracranial disease of the carotid arteries. However, in many studies dealing with cerebral ischaemia, information about the status of the vertebrobasilar arterial system is lacking. Since the vertebral arteries are often as large as, and sometimes larger than, the internal carotid arteries at the point where they penetrate the dura, and since they supply the vital centres of the brain stem 
this omission is remarkable. A probable reason is that the vertebral arteries take a rather inaccessible course for diagnostic investigation and surgical treatment. Another important reason may be that vertebral artery obstructions are less straightforward than obstructive lesions in the carotid artery, and the haemodynamics of vertebrobasilar arterial insufficiency are more complicated. However, the most important reason is that physicians have failed to comprehend the frequency of atherosclerosis of the innominate, subclavian, and vertebral arteries and its relation to cerebral ischaemia. Reports on the outcome of vertebrobasilar transient ischaemic attacks are less consistent than those of the anterior circulation. Consequently, there is a lack of consensus among clinicians on the management of patients with vertebrobasilar arterial disease.

Inadequacy of angiographic criteria for the interpretation of obstructions of the vertebral arteries, difficulties of the radiological technique, and the sometimes dramatic complications following vertebral angiography all contribute to the limited knowledge of the clinical significance of atherosclerosis of the vertebrobasilar arterial system. It has been reported that non-invasive Doppler techniques are of help in making an individual estimation of the severity of disease of the innominate, subclavian, and vertebral arteries. Quantitative information, however, is difficult to obtain. The main problems are the investigation of the proximal part of the vertebral artery and the differentiation of severe stenosis, occlusion, hypoplasia and aplasia. At present no reports are available about the accuracy of ultrasonic duplex scanning of the innominate, subclavian and vertebral arteries. Ultrasonic duplex scanning is a combination of two-dimensional imaging of the vessels of interest and Doppler measurement, using a single gated pulsed system. It provides the advantage of either technique used independently and in addition to audible interpretation real-time spectral analysis of the Doppler signals is used to evaluate the degree of disease. The ultrasonic image of the arteries is an anatomical guide by which one is informed about the exact location and course of the vessels of interest. The investigation of the vessels of the aortic arch, and the ostium and the proximal part of the vertebral arterty is usually not a problem. It has the additional advantage of investigating the vertebral artery in its bony canal until it loops around the atlas.

The purpose of this study is to evaluate ultrasonic duplex scanning in tracing obstructive disease of the innominate, subclavian and vertebral arteries and in particular to assess its accuracy and clinical value. The results are compared with the results of contrast arteriography and they are reviewed separately for each of the three vessels mentioned above.

It is also investigated whether there is any relation between different intracranial arterial distribution patterns in selective carotid and vertebral contrast 
arteriography and abnormal spectral wave forms in the vertebral arteries in patients with severe multi-level atherosclerotic disease.

In addition the usefulness of ultrasonic duplex scanning of the cervical part of the vertebral artery in relation to degenerative changes of the uncovertebral articulations is evaluated.

Finally, the significance of ultrasonic duplex scanning of the vertebral artery in the event of severe, obstructive disease in the intradural segment of this artery is evaluated.

In this study 2770 duplex examinations have been performed on $1972 \mathrm{pa}-$ tients between May 1981 and October 1984. We started with ultrasonic duplex scanning of the carotid arteries in May 1981. After a few months we realized that the investigation of the innominate, subclavian, and vertebral arteries with the duplex scanner was possible in most of the patients. Because of our increasing interest in atherosclerosis of the extracranial part of the cerebral arteries of the posterior circulation in both symptomatic and asymptomatic patients, a routine investigation of the innominate and subclavian arteries and the prevertebral segment of the vertebral artery was carried out. In the event of a possible history of vertebrobasilar insufficiency we also investigated the cervical segment of the vertebral artery up to the atlas. 

PART 1. LITERATURE STUDY

A man is as old as his arteries.

P.J.G. Cabanis, c. 1800. 



\subsection{INTRODUCTION}

The blood supply to the brain and the cranial part of the spinal cord is derived from the great vessels which arise from the aortic arch in the superior mediastinum: the brachiocephalic trunk, and the left common carotid and subclavian arteries. On both sides the vertebral artery is usually the first branch of the subclavian artery. It runs up the transverse foramina of the upper cervical vertebrae, enters the skull through the foramen magnum and connects with its counterpart on the other side to form the vertebrobasilar arterial system.

Any discussion on disease of the vertebrobasilar arterial system should begin with the origin of this vascular system, because obstructive disease, ulcerative plaques or anatomical anomalies anywhere in this part of the cerebrovascular tree may produce stroke or symptoms of insufficiency. For a good understanding of the possibilities and the limitations of ultrasonic duplex scanning of the vessels of the arch of the aorta and vertebral arteries, knowledge of the normal anatomy and anatomical variations of these vessels is indispensable.

This chapter contains a review of the anatomy of the arch vessels as well as the vertebral and basilar arteries. Besides the normal anatomy, anatomical anomalies are briefly described. As the examination of the carotid system falls beyond the scope of this study, the anatomy of the common carotid arteries is only briefly mentioned and not discussed in detail. To estimate symptoms of insufficiency of the vertebrobasilar arterial system, a survey of those parts of the central nervous system which are supplied by branches of the vertebral and basilar arteries is also given in this chapter.

The vital role of collateral circulation and its interplay in cerebrovascular disease has now become an important diagnostic consideration. When evaluating symptoms of insufficiency of the vertebrobasilar arterial system, clinicians and technicians must be aware of the potential of collateral circulation and its influence on diagnostic tests, as for example ultrasonic duplex scanning. The potential collateral pathways of the vertebral arteries are discussed at the end of this chapter.

\subsection{VASCULAR ANATOMY}

The arch of the aorta begins behind the manubrium sterni at the level of the upper border of the second right sternocostal articulation, and at first runs upwards and backwards to the left of the trachea. It is then directed back- 

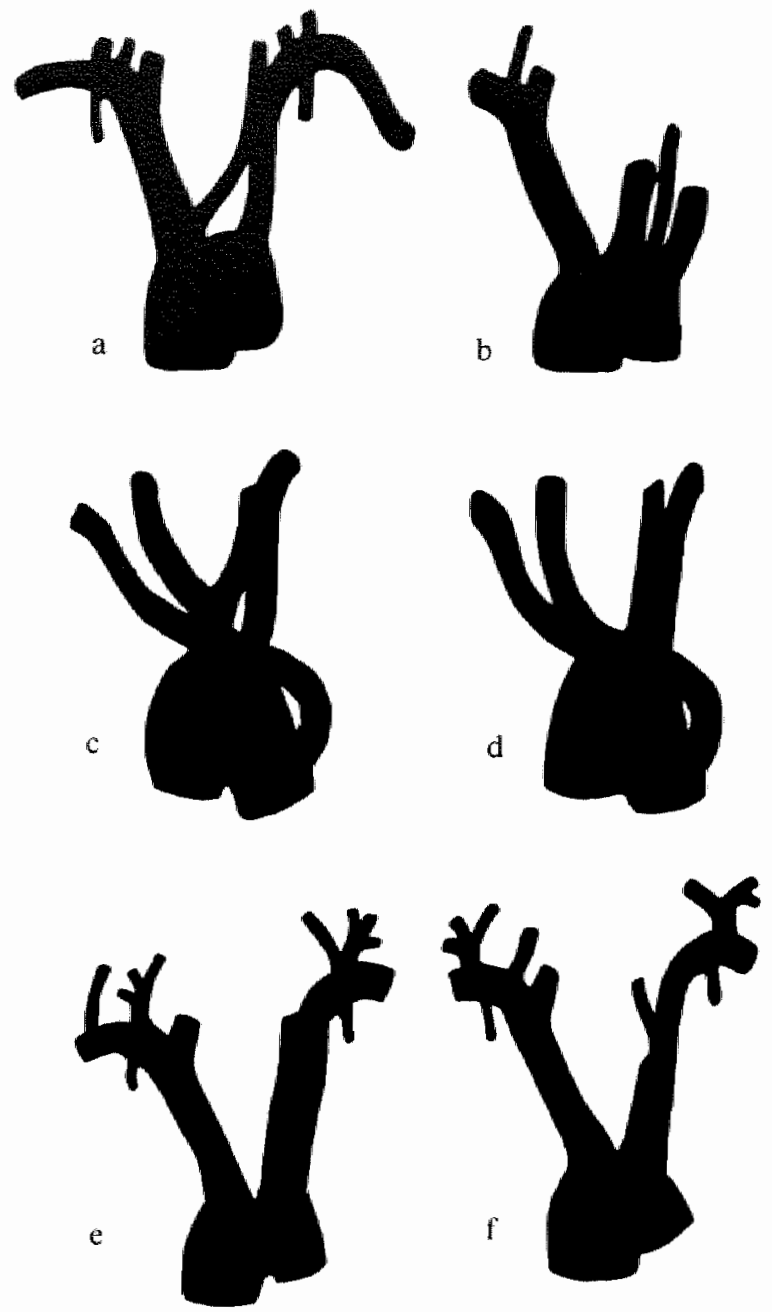

Figure 2.2/1

Some anatomical variations of the vessels of the arch of the aorta and vertebral arteries. 
wards on the left side of the trachea. Finely it passes downwards on the left side of the body of the fourth thoracic vertebra, at the lower border of which it is continuous with the descending aorta. Its termination corresponds to the sternal extremity of the second, left costal cartilage. It thus has two curvatures: one with its convexity upwards, the other with its convexity forwards and to the left. Its upper border is usually about the level of the middle of the manubrium sterni, but it may be considerably higher or lower than this.

Anatomical variations of the aortic arch may result in an abnormal origin and location of its branches. They are briefly summarized here and some of them are shown in figure $2.2 / 1$. Sometimes the aorta arches over the root of the right lung instead of that of the left lung. This right aortic arch passes downwards on the right side of the vertebral column. In this condition, which is normal in birds, there is usually a transposition of the thoracic and abdominal viscera. Less frequently, after arching over the root of the right lung, the aorta passes behind the oesophagus to gain its position on the left side of the vertebral column. This anatomical pecularity is not associated with transposition of the viscera. In this case the left subclavian artery arises as the last branch from that arch, and crosses behind the oesophagus en route to the left upper extremity.

The aorta occasionally divides, as in some quadriceps, into an ascending and descending trunk. The former is directed vertically upwards, and subdivides into three branches to supply the head and the upper limbs. Sometimes the aorta subdivides near its origin into two branches, which soon reunite. In this condition, which is normal in reptiles and is due to persistence of a part of the right dorsal aorta, the oesophagus and the trachea usually pass through the interval between the two branches.

Three branches arise from the upper aspect of the aortic arch: the brachiocephalic trunk, the left common carotid and the left subclavian arteries. At their origins the distance between these vessels varies. The most frequent variation in this respect is the approximation of the left common carotid artery to the brachiocephalic trunk. The branches may arise from the com= mencement of the arch or upper part of the ascending aorta.

The number of primary branches may be reduced to one. More commonly there are two (fig. 2.2/1a): in 7\% of the cases the left common carotid artery arises from the brachiocephalic trunk (White, 1969). More rarely the common carotid and subclavian arteries of the left side arise from a left brachiocephalic trunk. But the number of branches may increase to four, because the right common carotid and the subclavian arteries may arise directly from the aorta. In most of these cases the right subclavian artery arises from the left end of the arch and passes to the right behind the aorta. Daseler and colleagues (1959) found this anomaly in $0.25 \%$ of the 400 autotopsy specimens 
in their series (fig, $2.2 / 1 \mathrm{~d}$ ). A more common variation, in which there are four primary branches, is the left vertebral artery arising from the arch of the aorta between the left common carotid and subclavian arteries (fig. $2.2 / 1 \mathrm{~b}$ ). The figures of this anomaly vary between 2.46\% (Daseler et al,, 1959) and 60\% (Bosniak, 1964 -a). Very rarely the external and internal carotid arteries arise separately from the aortic arch, the common carotid being absent on one or both sides. In a few cases both wertebral arteries originate directly from the arch of the aorta.

When the aorta arches to the right side, the arrangement of the three branches of the arch is reversed. There is a left brachiocephalic trunk, and the right common carotid and right subclavian arteries arise separately. In other cases, where the aorta takes its usual course, the two common carotid arteries may be joined in a single trunk and the subclavian arteries arise separately from the arch. In this condition the right subclavian artery generally arises from the left end of the arch. (fig. 2.2/lc).

Other arteries may branch from the arch of the aorta. Most common are one or two bronchial arteries and the thyroidea ima artery. The latter is a small and inconstant artery that ascends in front of the trachea to the isthmus of the thyroid gland. It occasionally arises from the aorta, the brachiocephalic trunk, or the right common carotid, subclavian or internall thoracic arteries.

The brachiocephalic trunk or innominate artery is the first and largest branch of the arch of the aorta. It is 4 to $5 \mathrm{~cm}$ in length and arises from the

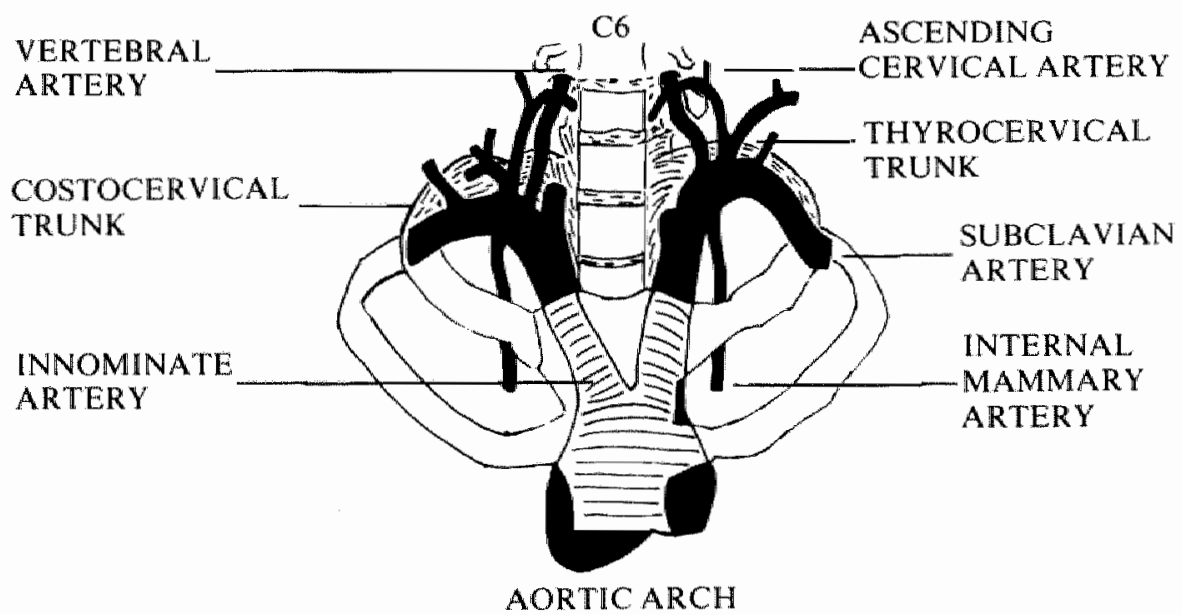

Figure 2,2/2

Normal location and arrangement of the aortic arch, arch vessels and branches from the subclavian arteries. 
convexity of the arch, posterior to the centre of the manubrium sterni (fig. $2.2 / 2$ ). It passes obliquely upwards, backwards and to the right, lying at first in front of the trachea and then on its right side. At the level of the upper border of the right sternal clavicular joint it divides into the right common carotid and subclavian arteries. The root of the innominate artery is crossed anteriorly by the left brachiocephalic and right inferior thyroid veins. On its right side are the right brachiocephalic vein and the upper part of the vena cava superior. The innominate artery is usually devoid of branches other than its terminal ones, but occasionally the thyroidea ima may arise from it, or it gives off a thymic of bronchial branch.

The principal arteries of the head and the neck are the two carotid arteries. They ascend in the neck as far as the upper border of the thyroid cartilage, where each divides into the internal and external carotid arteries. The common carotid arteries differ in length and in their mode of origin. The right common carotid artery begins at the bifurcation of the innominate artery behind the right sternoclavicular joint and is confined to the neck. The left common carotid artery springs from the highest part of the arch of the aorta immediately behind and to the left of the innominate artery. Its thoracic part ascends from the aortic arch to the level of the left sternoclavicular joint, where it is continuous with its cervical portion. The left common carotid artery varies in its origin more frequently than the right. In the majority of abnormal cases it arises together with the innominate artery. If that artery is absent, the two common carotid arteries arise usually by a single trunk. It is rarely joined with the left subclavian artery, except in the event of transposition of the aortic arch. The common carotid arteries usually have no branches. However, it may give origin to the vertebral, superior thyroid or its laryngeal branch, the ascending pharyngeal, the inferior thyroid or the occipital arteries.

The first part of the subclavian arteries differ from one another in their origin, length, course and relations (fig. $2.2 / 2$ ). The right subclavian artery originates behind the right sternoclavicular joint. It passes upwards and laterally to the medial margin of the anterior scalenus muscle. It ascends about two centimeters above the clavicle, but the height it reaches varies considerably. At its start it is behind the origin of the right common carotid artery. More laterally it is crossed by the internal jugular and vertebral veins. The left subclavian artery is the third branch of the arch of the aorta. It arises behind the left common carotid artery, usually at the level of the disc between the third and fourth thoracic vertebra. It ascends to the root of the neck and arches laterally as far as the medial border of the left anterior scalenus muscle. Within the thorax it is related anteriorly to the left common carotid artery and the commencement of the left brachiocephalic vein. 
The subclavian arteries can be represented by a broad line, convex upwards, drawn from the sternoclavicular joint to the middle of the clavicle. As a result of its origin from the arch of the aorta the left subclavian artery is more posterior in its first part than the right one, and, as a rule, it does not reach quite as high a level in the neck.

On both sides the vertebral artery is the first branch of the subclavian artery, arising from the upper and posterior wall of the first part of this vessel (fig. 2.2/2). Especially the origin of the left vertebral artery from the left subclavian artery may vary considerably in height (fig. $2.2 / 1 \mathrm{f}$ ). Sometimes the thyrocervical trunk is the first branch, the wertebral artery arises from the subclavian artery lateral to or as a common trunk with the thyrocervical trunk (fig. $2.2 / 1 \mathrm{e}$ ). There may be an anomalous origin of the vertebral artery from the aorta, innominate or common carotid arteries.

The vertebral artery is generally divided into four segments. The first (prevertebral) segment ascends upwards and backwards between the longest colli and scalenus anterior muscles to enter the transwerse foramina of the cervical spine at the level of the sixth cervical vertebra (fig. 2.2/2). This segment of the artery is usually 4 to 5 centimeters in length. Anteriorly it is related to the common carotid artery and the vertebral vein and is crossed by the inferior thyroid artery. Posteriorly, it is related to the transverse process of the seventh cervical vertebra. In many cases the vertebral artery shows a posteroinferior flat curve just distal to its origin from the subclavian artery. Occasionally the vertebral artery may enter the fifth, fourth or seventh cervical vertebrae. Branches which arise from the prevertebral segment of the vertebral artery are rare. Daseler and co-workers (1959) found the inferior thyroid artery to be a branch of the vertebral artery in $0.64 \%$ of the cases. In $0.81 \%$ of the cases the costocervical trunk arose as a branch from this part of the vertebral artery.

The second (cervical) segment has a straight course encased in the bony canal formed by the transverse foramina of the upper six cervical vertebrae. It pursues an almost vertical course as far as the transverse process of the axis, through which it runs upwards and laterally to the transverse foramen of the atlas. It is associated with a plexus of veins which unite to form the vertebral vein in the lower part of the neck. The artery passes anteriorly to the vertebral rami of the cervical nerves (C6-C2) and lies in apposition with each successive nerve. The nerve sheath is often grooved as the vessel passes upwards. On its medial aspect the vertebral artery bears an intimate relationship to the neurocentral joint. This is the junction of the centrum and lateral masses in development of the vertebrae, and is also known as Luschka's joint or the uncovertebral joint. There is disagreement on whether this is a true joint or not (Payne et al., 1957). The anatomical relationship of the vertebral artery to the 
uncovertebral joint is assumed to be important when the cervical vertebrae are effected by degenerative osteo-arthritic change.

The third (atlantic) segment issues from the transverse foramen of the atlas on the medial side of the rectus capitis lateralis. It curves horizontally backwards and medially around the articular process of the atlas, lying in a groove on the upper surface of the posterior arch of the atlas. The artery enters the vertebral canal by passing below the lower, arched border of the posterior atlanto-occipital membrane. This part of the artery is contained in the suboccipital triangle and is covered by the semispinalis capitis.

The fourth (intradural) segment pierces the dura and arachnoid mater, ascends in front of the roots of the hypoglossal nerve and inclines medially to

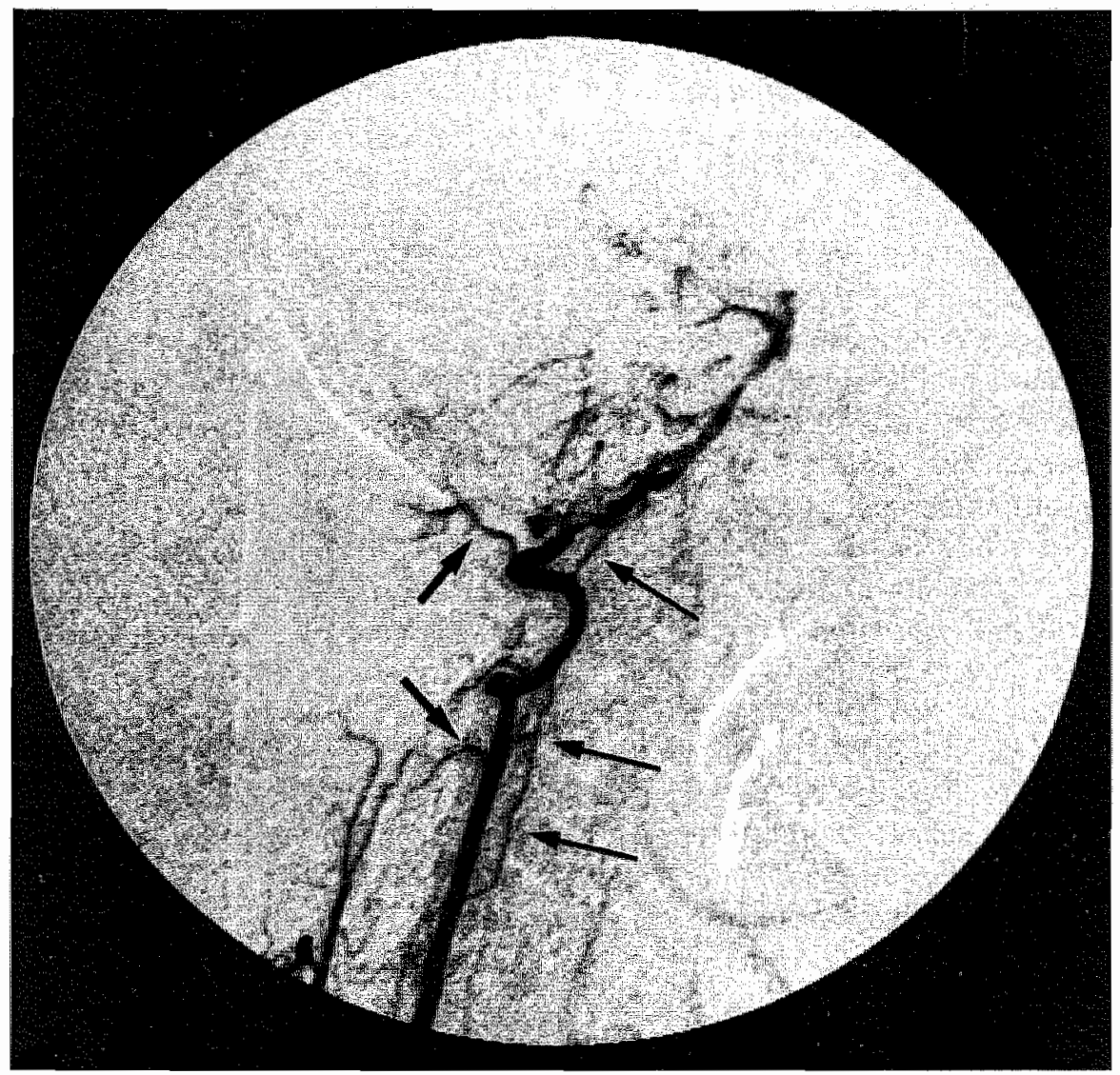

Figure 2.2/3

An intra-arterial digital subtraction angiogram of a right vertebral artery w/th seweral muscular branches $(\rightarrow)$ ) and a large anterior spinal artery $(\longrightarrow$ ). 
the front of the medulla oblongata where, at the lower border of the pons, it unites with the opposite artery to form the basilar artery.

The branches of the vertebral artery may be divided into two sets: cervical branches which arise in the neck, and cranial branches which arise from the intradural segment of the artery. These branches are briefly mentioned here from a proximal to a distal position.

The spinal branches arise from the cervical segment of the vertebral artery and enter the vertebral canal through the intervertebral foramina. They give rise to anterior and posterior radicular arteries which approach the spinal cord along the ventral and dorsal nerve roots. According to Lazorthes (1972) only two or three spinal branches of the vertebral artery actually vascularize the middle segments (C4-C6) of the cervical part of the spinal cord, the socalled radiculomedullary arteries. The other spinal branches terminate in the nerve roots (the radicular arteries sensu strictiori) or do not extend beyond the pial leptomeningeal arterial plexus (the radiculopial arteries).

The muscular branches arise from the cervical and atlantic segments of the vertebral artery as it curves round the lateral mass of the atlas. They supply the deep muscles of this region and anastomose with the occipital artery, and with the ascending and deep cervical arteries (fig. 2.2/3).

At the upper end of the second and third segments a small anterior and a larger posterior meningeal branch originate respectively. The former supplies the dura of the anterior margin of the foramen magnum, and the latter supplies the posterior rim of the foramen magnum, the falx cerebelli and the posteromedial portion of the dura of the posterior fossa. The posterior meningeal branch may extend superiorly to supply the posterior portion of the falx cerebri. The arteries supplying the meninges freely anastomose among themselves and with their counterparts from the opposite side of the skull.

The posterior inferior cerebellar artery is the largest branch of the vertebral artery, but is frequently absent. Usually it springs from the intradural segment of the vertebral artery. Occasionally it arises from the basilar artery. It finally divides into a medial and a lateral branch. The medial branch runs backwards between the cerebellar hemisphere and the inferior vermis, supplying branches to both; the lateral branch supplying the undersurface of the hemisphere, as far as its lateral border, and anastomoses with the anterior inferior cerebellar and superior cerebellar branches of the basilar artery. The trunk of the posterior inferior cerebellar artery supplies the medulla oblongata and the choroid plexus of the fourth ventricle. It also sends a branch upwards lateral to the tonsil to supply the dentate nucleus of the cerebellum. The area supplied in the medulla oblongata lies dorsal to the olivary nucleus and lateral to the nucleus and emerging fila of the hypoglossal nerve.

The posterior spinal artery may arise from the vertebral artery at the side of 
the medulla oblongata, but most frequently originates from the posterior inferior cerebellar artery. It passes backwards and then descends as two branches, one in front and the other behind the dorsal roots of the spinal nerves.

The anterior spinal artery is a small branch, which arises near the termination of the vertebral artery. It descends in front of the medulla oblongata and unites with its counterpart of the opposite side. The single trunk thus formed descends on the front of the spinal cord. The anterior spinal artery supplies the medial part of the medulla oblongata and the main part of the superior cervical segments ( $\mathrm{Cl}-\mathrm{C} 4)$.

The medullary arteries are several minute vessels which originate from the vertebral artery and its branches and are distributed to the medulla oblongata.

The basilar artery is formed by the junction of the two vertebral arteries at the level of the pontomeduliary sulcus between the two abducens nerves. It extends from the lower to the upper border of the pons, where it divides into two posterior cerebral arteries.

The pontine arteries are numerous, small, penetrating branches of the basilar artery, and can be divided into medial and lateral groups. The lateral group not only supplies the pons, but also the ventrolateral aspect of the cerebellar cortex. Towards its upper end, the basilar artery gives off several penetrating branches that supply the inferior portion of the midbrain.

The anterior inferior cerebellar artery arises from the lower part of the basilar artery. At first it passes ventrally, then horizontally across the inferior portion of the pons, and finally through the cerebellar pontine angle cistern, along with the facial and vestibulocochlea nerves to reach the internal auditory meatus. It then loops on the antero-inferior surface of the cerebellum, supplying the middle cerebellar peduncle and adjacent areas of the cerebellar hemisphere. In its pontine course it gives rise to many pontine perforating branches. The internal auditory artery may arise directly from the basilar artery but is more often derived from the anterior inferior cerebellar artery. It enters the internal auditory canal, contributing to the blood supply of the dura mater of the canal and sending branches to the cochlea, the labyrinth and the horizontal portion of the facial nerve.

The superior cerebellar artery arises from the basilar artery, just proximal to its termination. It passes laterally just caudal to the oculomotor nerve to circle the cerebral peduncle or upper pons just below the trochlear nerve. It sends multiple branches to the midbrain, adjacent pons and the superior cerebellar peduncle. On the upper surface of the cerebellum it divides into branches which ramify in the pia mater, supplying this aspect of the cerebellum. In addition branches are given off to the pineal body, the superior medullary velum, and the tela choroidea of the third ventricle. 
The posterior cerebral artery arises from the terminal bifurcation of the basilar artery. While this artery predominantly supplies the supratentorial structures of the brain, it also contributes to the blood supply of the upper part of the midbrain. The proximal segment of the posterior cerebral artery from its origin to its junction with each posterior communicating artery constitutes a distinctive arterial segment, called the mesencephalic artery. From this segment of the posterior cerebral artery arise complex penetrating vessels destined chiefly for the thalamus, subthalamus, rostral midbrain-subthalamic junction, and adjacent structures. It should be noted that the posterior cerebral artery also supplies the visual area of the cerebral cortex.

\subsection{COLLATERAL PATHWAYS}

Once it was believed that arteries in the brain were end arteries. Now it is known that capillary and precapillary anastomoses are common. To further appreciate these collateral pathways, it should be noted that there are two types of arteries supplying the brain. The more important in terms of neuronal function and nutrient supply are the penetrating arteries. However, it is the diffuse superficial arteries spreading over the entire surface of the central nervous system through which collateral circulation takes place. The circle of Willis and the major arterial trunks are included in this superficial system. Second only to the circle of Willis is the complex of intracranial-extracranial anastomoses. For the vertebrobasilar arterial system this network connects the occipital branch of the external carotid artery, the deep cervical artery and the ascending cervical artery with branches of the vertebral artery. When evaluating ultrasonic duplex scanning of the vertebral arteries one should be conversant with the anatomy of these potential collaterals.

The thyrocervical trunk is a short and wide vessel which arises from the upper and front part of the first segment of the subclavian artery, usually a half to two centimeters lateral to the origin of the vertebral artery. It divides almost immediately into three branches: the inferior thyroid, the suprascapular and superficial cerwical arteries. Of these the former runs upwards in front of the medial border of the scalenus anterior. It then turns medially in front of the vertebral vessels and behind the carotid sheath. It finally descends on the longus colli muscles to the lower border of the lobe of the thyroid gland. The ascending cervical artery is a small branch which arises from the inferior thyroid artery as that vessel turns behind the carotid sheath. It ascends on the anterior tubercles of the transverse processes of the cervical vertebrae in the interval between the scalenus anterior and the longus capitis muscles. Besides small branches to the muscles of the neck it sends off one or two spinal branches into the vertebral canal through the intervertebral foramina. It anastomoses with muscular branches of the vertebral artery and with the occipital and deep cervical arteries. 
The costocervical trunk arises from the back of the second part of the subclavian artery on the right, but from the terminal portion of the first part of that vessel on the left. It arches backwards above the cervical pleura to the neck of the first rib and divides into the superior intercostal and deep cervical. arteries. Occasionally the latter is a separate branch from the subclavian artery. Passing backwards between the transwerse process of the seventh cervical vertebra and the neck of the first rib it gives off a spinal branch which enters the vertebral canal through the foramen between the seventh cervical and first thoracic vertebrae. It ascends in the back of the neck, between the semispinalis capitis and cervicis muscles as high as the second cervical wertebra. It supplies the adjacent muscles, and anastomoses with the deep division of the occipital artery and the muscular branches of the vertebral artery.

The occipital artery arises from the back of the external carotid artery, opposite to the facial artery. It passes backwards, crossing in its course the internal carotid artery and the internal jugular vein. Reaching the interval between the transwerse process of the atlas and the mastoid process of the temporal bone, it comes into contact with the laterall border of the rectus capitis lateralis. It then runs in the occipital groowe on the temporal bone, and finally turns upwards and pierces the cranial attachment of the trapezius and sternocleidomastoid muscles. The descending branch arises from the occipital artery as the latter lies on the obliquus capit is superior and divides into superficial and deep branches. The deep branch descends between the semispinalis capitis and cervicis muscles, and anastomoses with the deep cervical artery, and with the vertebral artery. 


\section{PHYSIOLOGY AND PATHOPHYSIOLOGY OF CEREBRAL CIRCULATION}

\subsection{INTRODUCTION}

The human brain may be considered as a biological computer, which is composed of billions of cells and circuits that make it the most sophisticated organism in the universe that we know of. This computer has only one severe problem: it is extremely dependent on a continuous and adequate supply of oxygen and glucose. It may be considered to be obligatory aerobe, that is, it derives all of its energy from the oxidative metabolism of glucose. In order to maimtain this condition oxygen must be present and of sufficiently high concentration in the brain tissue. A relative high average capillary oxygen tension of approximately $60 \mathrm{~mm} \mathrm{Hg}$ is necessary for the maintenance of oxygen tension above the critical value of 1 to $2 \mathrm{mmHg}$ in cells furthest away from the capillaries. There is still disagreement concerning the question whether oxygen is supplied to the tissue only by diffusion or that glial cells play some role in the oxygen transfer from the blood to the nerve cells (McHenry, 1983).

Although the brain represents only approximately $2 \%$ of body weight, at rest it utilizes 20-25\% of the oxygen inspired and needs about $80 \mathrm{mg}$ glucose per minute. As cerebral energy metabolism takes place in the mitochondria of the celis, it is understandable that the demand for oxygen and glucose differ from one region to another. Roy and Sherrington showed in 1890 the fundamental importance of cerebral metabolism as the primary factor in the regulation of the cerebral circulation. Recent studies on cerebral metabolism show that focal changes of blood flow occur in a variety of physiological circumstances. For example, one may demonstrate an increase in regional cerebral blood flow in the occipital cortex by retinal light stimulation, and regional metabolism in the precentral motor cortex increases with exercise of the opposite extremity (Lassen et al., 1978, Olesen, 1971). These differences, however, are relatively small and as a whole brain metabolism and cerebral blood flow are only slightly enhanced by cerebral activation.

The normal average blood flow through the brain tissue is about $60 \mathrm{ml}$ $/ \mathrm{min} / 100 \mathrm{gram}$. For the total brain this amounts to $900-1100 \mathrm{ml} / \mathrm{min}$., which is about $15 \%$ of the resting cardiac output. The above mentioned value is a mean value, regional differences are present. For example, blood flow is considerably higher in the grey than in the white matter of the brain.

\subsection{REGULATION OF CEREBRAL CIRCULATION}

The regulation of cerebral circulation is based on the concept of cerebral 
autoregulation. This term refers to the fact that within a certain range blood flow remains constant despite changes in perfusion pressure. Strictly defined, autoregulation is restricted to control by intrinsic mechanisms, that is, to mechanisms remaining in a denervated organ without autonomic control. Most of the observations of constant cerebral blood flow during changes in perfusion pressure were made with autonomic innervation of the cerebral vessels intact. Carefully defined, the results of these studies do not appropriately describe autoregulation. However, there is strong evidence that the regulatory mechanisms are almost completely independent of structures outside the cranial cavity.

Cerebral blood flow is determined by the pressure gradient between the input and output of the system and by the cerebrovascular resistance. Under physiological conditions the perfusion pressure of the brain is mainly determined by the mean arterial blood pressure; the intracranial and venous pressures having a low value. The resistance of the cerebral vasculature is determined by the resistance in the capillaries and in the arterioles. The former can be assumed to be constant because in the brain there are no possibilities of shutting of or opening important parts of the capillary system. Because of the constant high metabolic need for the neurons such a mechanism could not exist in the brain. Thus, the cerebrovascular resistance is mainly located in the arterioles. These vessels have the ability to dilate or to constrict within a certain range so that the total cerebrovascular resistance varies between certain limits. This mechanism makes it possible for the brain to maintain a constant cerebral blood flow which is to a large extent independent of the mean arterial blood pressure.

The most important determinant of cerebral blood flow is the carbon dioxide tension in arterial blood. Although the occurrence of cerebral vasodilatation in response to increased arterial carbon dioxide tension, and of cerebral vasoconstriction in response to decreased arterial carbon dioxide tension can be constantly demonstrated, the exact mechanism involved is not known. It has been postulated that change in $\mathrm{pH}$ of the extracellular fluid around the arterioles and not the change in carbon dioxide tension itself is the stimulus by which arterial carbon dioxide controls cerebral blood flow. The physiological meaning might be that acidosis caused by insufficient metabolism gives a vasodilatation and a better perfusion to the tissue.

Arterial oxygen tension has an opposite effect. However, the vascular responses to changes in arterial oxygen tension are less pronounced than the responses caused by changes in arterial carbon dioxide tension. Other factors such as perivascular potassium and bicarbonate concentration play a role in the control of vascular diameters and cerebral blood flow. However, their interaction with hydrogen ions is not clearly understood. 
Cerebral vessels are innervated by both sympathetic and parasympathetic nerve fibers and present evidence suggests that the autonomic system can significantly alter cerebral blood flow. The conditions under which these mechanisms are active have yet to be clarified. For example, the adjustment of cerebral blood flow to postural changes has been postulated to be reflexogenic in origin, but baroreceptor reflexes do not seem te be necessary for the tone of the cerebral vasculature (D'Alecy, 1974).

Beside these metabolic and neurogenic factors an important mechanism involved in the regulation of cerebral blood flow is described by Bayliss in 1902. In this mechanism the smooth muscle fibres in the arteriolar wall react directly to transmural pressure changes. When pressure in an artery suddenly rises the arteriole constricts. When the pressure falls the vessel dilates. These observations were made in vitro, thus metabolic and neurogenic influences could be excluded.

To summarize, it is very likely that the mechanisms which play a role in the regulation of cerebrall blood flow are working together in cerebral autoregulation. Most of the factors mentioned are located in the brain itself. The cerebral vasculature shows an extensive autonomic innervation, but the exact place of neural control in the complex mechanism of cerebral autoregulation is not yet clarified.

\subsection{PATHOGENESIS OF NEUROLOGICAL DEFICITS}

Much of the disagreement about the aetiology of cerebral ischaemic attacks has been due to the attempt to identify a single factor accounting for all attacks, whereas many causes are likely to be involved. The association of transient ischaemic attacks with occlusion or a tight stenosis of the carotid of vertebral arteries suggests that a reduced perfusion might be enough to cause transient focal symptoms. This haemodynamic view of transient ischaemic attacks was advocated by Denny-Brown and co-workers (1957), who produced a neurological deficit in the monkey by inducing hypotension after occluding the middle cerebral artery. That this mechnism can operate in patients and cause transient, focal neurological deficit is well established (Eastcott et al., 1954, Shanbrom et al., 1957, Ruff et al., 1.981 and Riley et al., 1981). A postural hypotension, a haemodynamically significant cardiac arrythmia or a cardiac decompensation induced by exercise may cause a transient ischaemic attack.

On the other hand systemic hypotension appears to be a relatively rare cause of cerebral ischaemia since blood pressure measured during attacks is usually normal. Attempts to reproduce transient ischaemic attacks by reduction of systemic blood pressure are ineffective or, if the reduction is severe, loss of consciousness occurs due to generalised cerebral ischaemia (Kendell et 
al., 1963, Reed et al., 1973). This is understandable since cerebral autoregulation remains effective for a long time even in the presence of arterial disease. The precise range of blood pressure which will reduce blood flow in one region of the brain while maintaining an adequate flow to other regions is a narrow one. Usually focal symptoms are likely to develop only when a local vascular obstruction makes one cerebral territory more vulnerable to a drop in perfusion pressure. Ruff and co-workers (1981) demonstrated that acute lowering of blood pressure through antihypertensive medication, postural changes or cerebral angiography was associated with transient focal neurological deficit in hypertensive patients with haemodynamically significant common or internal carotid artery stenosis. In some of these patients the angiography also showed intracranial atherosclerosis in a distribution which corresponded to the focal neurological deficit present during the transient ischaemic attacks. In their study transient ischaemic attacks were related to hypotension in only $5.3 \%$ of the patients. The incidence, however, rose significantly to $25 \%$ in patients with haemodynamically significant carotid artery stenosis and to $44 \%$ in patients with carotid artery stenosis and hypertension. Although the pathophysiological basis for the relationship between hypotension and transient focal cerebral deficit cannot be derived from their work, the attacks may be due to the fact that the autoregulation reserve is exhausted by compensation for reduced pressure in the circle of Willis, so that any additional haemodynamic crisis can provoke symptomatic cerebral ischaemia. Attacks of this type more often affect the vertebrobasilar circulation (Ross Russell, 1983) and this may account for the less serious prognosis of transient ischaemic attacks in this territory.

Different haemodynamic mechanisms can cause the transient appearance of focal neurological deficit. Derangement of the control of cerebral circulation has been demonstrated in obstructive cerebrovascular disorders. The normal responsiveness of the cerebral vasculature to carbon dioxide may be reduced or lost, or paradioxical responses may occur. In such cases it is postulated that the ischaemic area is no longer responsive to carbon dioxide because of the accumulation of acid metabolites and the presence of hypoxia. The normal surrounding tissues are still reactive to carbon dioxide. If the vessels in the perifocal area dilate this will cause a reduction in cerebrovascular resistance and in local perfusion pressure. This, in turn, produces a decrease in flow through the vessels of the ischaemic area whose resistance can not be reduced further. This phenomenon has been called the intracerebral steal syndrome.

In the aftermath of a cerebral infarction the normal mechanism of autoregulation of cerebral blood flow, which maintains a level of blood flow over a wide range of mean arterial pressures, is often impaired. Consequently, cerebral blood flow becomes pressure-dependent at all levels of perfusion pres- 
sure. There is evidence that such a dysautoregulation may persist for days to weeks after the infarction.

Regional losses of responsiveness to carbon dioxide or of autoregulation can occur independently of each other. That is, a region may show loss of autoregulation and still be responsive to carbon dioxide or vice versa. The former, however, is more frequently observed.

An unusual type of haemodynamic crisis may affect the vertebrobasilar territory when there is a localised proximal occlusion or severe stenosis of the innominate or subclavian artery. The normal pressure gradient in the vertebral artery is reversed, the pressure being lower in the neck than in the head. Blood passes up in one vertebral artery and down in the other into the arm. This so-called subclavian steal syndrome will be discussed in more detail in chapter 5.4 .

Flow problems, however, can only explain a minority of cerebral ischaemic attacks. Symptoms suggesting a syncopal state are rare in patients with focal neurological deficit and the attacks are rarely related to exercise, posture or head position. Embolism has now become accepted as an important cause of transient ischaemia, especially in the carotid territory. The evidence for thromboembolism as the main cause of transient ischaemic attacks is largely clinical. More than twenty years ago, Fisher (1959) and Ross Russell (1961) described the passage of emboli through the retinal circulation in patients during attacks of amaurosis fugax. In 1964 Gunning and co-workers showed in an extensive paper the connection between mural thrombosis of the internal carotid artery and cerebral or retinal emboli. These direct observations are rare and the presence of a potential source for embolism rather than visible embolism constitutes the evidence for this process in most instances. Examination of resected specimens of carotid artery from patients with transient ischaemic attacks has confirmed the frequent presence of intraluminal thrombus, friable atheromatous debris, or intraluminal haemorrhage at the site of the atheromatous lesion, especially in those patients who have experienced recent attacks (Harrison et al., 1977, and Imparato et al., 1979). In patients with unilateral atherosclerotic lesion of the carotid artery the symptoms are significantly more frequent on the side of the lesion than on the contralateral side (Drake et al., 1968).

Apart from the neck vessels the heart is a very important source of embolism causing cerebral infarction. Some autopsy and clinical studies (Jorgensen et al. , 1966, B $\|$ ackwood et al., 1969, Gautier et al., 1975) suggest that cerebral infarcts can be attributable to embolism from the heart in up to $50 \%$ of the cases. The commonest cardiac lesions encountered clinically are atrial fibrillation and mitral stenosis, and especially a combination of these two.

There is evidence that embolism from the neck vessels predominate in the 
pathogenesis of transient ischaemic attacks but having a smaller role in completed stroke (Ueda et al., 1980, De Bono et al., 1981). On the contrary stroke victims more frequently have a cardiac source of embolism, and neck vessel disease is relevant because of the development of carotid occlusion by thrombosis of a local stenosis, rather than because of artery to artery embolism. However, patients with mitral valve prolapse without atherosclerosis of the cerebral arteries may show transient ischaemic attacks as well as cerebral infarction (Barnett et al., 1976, 1980, Barnett, 1982, Hanson et al., 1980). Moreover, intracranial atheroma is more common in stroke patients than in those with transient ischaemic attacks.

The heart lesions involved in completed stroke may more often be those associated with atrial or ventricular thrombus. It is likely that some of these differences are related to the different size and structure of emboli. Fibrin formation stabilizes larger thrombi developing in the atrium and emboli derived from heart chambers may well be more stable than those which cause transient ischaemic attacks.

The differences in site of origin and composition of emboli may have an important clinical relevance. Atheromatous embolism from an ulcerated carotid lesion would not be expected to respond to antithrombotic therapy, while fibrin stabilized thrombi in a heart chamber might be more sensitive to anticoagulation. Most of the above mentioned data has been collected in the context of cerebral ischaemic attacks in the carotid territory since they have been studied more intensively. However, there is some evidence that in the pathogenesis of ischaemic attacks in the vertebrobasilar territory flow problems are more commonly involved, ulceration of an atheromatous lesion of vertebral arteries being infrequent (Ross Russell, 1983). 


\section{THE ROLE OF VERTEBRAL ARTERY DISEASE IN THE PATHOGENESIS OF CEREBRAL ISCHAEMIA}

\subsection{INTRODUCTION}

It must be emphasized here that atheromatous plaques by themselves do not produce any symptoms and that at present no method is available to indicate those cases in which symptomatic atherosclerotic cerebral vascular disease will develop in the future. Because atheroma have been seen as incidental findings in the arteries of young adults or even children, atherosclerosis is known to begin in early life. It usually does not become symptomatic untill later because the crippling effects of the disease are not due to the sclerosis, but are secondary to the decrease in the amount of blood supplied to the tissues.

The basic degenerative process of atherosclerosis is a diffuse one. Usually, however, it involves somatic and visceral arteries in various degrees. The relative incidence of distribution of atherosclerotic lesions in man is largely lacking. Necropsy studies dealing with the entire arterial system are meager. Most studies have focused on either the peripheral arterial tree or individual visceral arteries.

Although coronary artery disease, renal or retinal arteriopathies, aortailiacal obstructive disease, and carotid or vertebrobasilar atherosclerosis are all conditions which may exist without signs of the others, these processes are all interrelated and impairment of functions of one system by the atherosclerotic process may cause malfunction in another. In the presence of underlying cerebral atherosclerosis, for example, cardiac decompensation secondary to coronay artery disease may precipitate cerebral vascular insufficiency. Other factors that enter the clinical picture produced by cerebral atherosclerosis are pulmonary diseases which reduce oxygenation of the blood, fluctuations in systemic blood pressure, variations in cardiac output, and abnormalities in the viscosity of blood and its content of haemoglobine, oxygen and glucose.

\subsection{ATHEROSCLEROSIS OF THE INNOMINATE, SUBCLAVIAN, AND VERTEBRAL ARTERIES}

One of the most striking features of atherosclerosis of the carotid and vertebrobasilar arterial systems is its patchy distribution. Some sites within an arterial segment have a high incidence of lesions, where as other areas are virtually free from disease. The contrast between the carotid sinus and the cervival carotid artery immediately distal to it is the most outstanding example. The high incidence of proximal lesions in the innominate, subclavian, vertebral and left common carotid arteries is also a significant finding and 
contrasts with the right common carotid artery where lesions are most commonly found in the distal part of the artery. It is not easy to understand these differences of localisation of atherosclerotic lesions. There is no consistent difference in structure of the arterial wall between sites with a high and a low atheroma prevalence. For example, there is no structural difference between the proximal and distal segment of the vessels of the aortic arch, which have marked discrepancies in frequency of atheromatous lesions. Dynamic factors as a result of blood flowing under pulsatile pressure through the arterial system may be of significance by producing local areas of turbulence, flow separation and by decreasing sheer stress on the vessel wall (Stehbens, 1959, LoGerfo et al., 1981 and Bharadvaj et al., $1982 \mathrm{a}, \mathrm{b}$ ).

In contrast to the focal deposition of atherosclerotic lesions in the innominate, subclavian and carotid arteries atherosclerotic plaques tend to be scattered rather indiscriminately along the vertebal arteries (Hutchinson et al., 1956, Fisher et al., 1965). The degree of sclerosis may vary from a localized plaque with little or no stenosis to diffuse involvement of the artery with atheroma in all its parts. Most studies have such an insufficient number of cases that we are unable to speak with certainty of a typical location of the lesion; nevertheless the site of prevalence of a severe stenosis and occlusion is at the origin of the vertebral artery from the subclavian artery (Hutchinson et al., 1956, 1957, Schwartz et al., 1961, Fisher et al., 1965, Thevenet, 1979). The relatively narrow calibre of the vertebral arteries allow them to be markedly narrowed by small localized fibrous plaques which are common in these vessels. Often it appears to be a contiguous involvement of the vertebral artery when the subclavian artery is affected by atheroma. The obstructing mass is the result of a combination of deposit in the subclavian and in the vertebral artery, the former accumulating in a ring about the orifice and leading to constriction of the lumen. Sometimes the atherosclerotic deposition extends upwards into the vertebral artery for $S$ to $10 \mathrm{~mm}$.

Another principal site of atherosclerotic changes in the vertebral arteries is the intradural segment of the artery just proximal to the conjunction with the artery of the other side. In the third decade of life, Moossy (1966) already found small plaques in the vertebral arteries a few millimeters distal to the point where these vessels enter the intracranial cavity. Castaigne and co-workers (1973), in their study on arterial occlusions in the vertebrobasilar arterial system, found 25 vertebral artery occlusions in 22 patients. In $68 \%$ the occlusion resulted from thrombosis superimposed on a pre-existent stenosis. The site of the primary thrombosis was in the intradural segment of the vertebral artery in 12 cases while in only four cases the prevertebral segment was involved.

Atherosclerotic lesions in the cervical segment of the vertebral artery are commonly complicated by displacement of the vessel by exostoses and spur 
formation due to cervical spondylosis. Lesions in this segment of the artery are variable in appearance (Moossy, 1966), but when they occur they may show, at fairly regular intervals, a ladder-like arrangement. Usually they give rise to a pathognomonic complex of symptoms. Lesions in this segment of the vertebral artery are discussed in more detail in chapter 5.3.

There seems to be no increased tendency to atheroma in the tortuous course of the vertebral artery around the axis and atlas (Hutchinson et al., 1956). Recently Ringelstein (1984) found in a continuous wave Doppler sonographic study on 4,468 patients a significant stenosis in the atlantic segment of the vertebral artery in only $0.16 \%$ of the patients. This segment of the artery is more susceptible to forceful manipulations of the neck, as with chiropratic adjustments (Krueger et al., 1980, Robertson, 1981, Sherman et al., 1981).

In the same way atherosclerosis involves the basilar artery (Meyer et al., 1960), its penetrating branches, the long circumferential cerebellar arteries and the posterior cerebral arteries. However, it is not possible to obtain information about this part of the vertebrobasilar arterial system by ultrasonic duplex scanning and therefore the clinical syndromes which result from obstructive lesions of these vessels are beyond the scope of this study. The major types of vascular pathology of the intracranial part of the vertebrobasilar arterial system and the delineation of several clinically recognizable syndromes have been discussed by a variety of investigators (e.g. Fisher, 1965, 1967, Fisher et al., 1971, Castaigne et al., 1973, Caplan et al., 1975, Thompson et al., 1978, Caplan, 1980, 1981).

An important fact of atherosclerosis of the vertebral arteries to be emphasized here is the uncommon occurrence of ulcerative lesions. Fisher and colleagues (1965) found ulceration in the vertebral system in only $4 \%$ of their cases while at the same time it was directly related to the severity of the atherosclerosis. Schwartz and colleagues (1965) investigated the innominate, subclavian, and vertebral arteries in a consecutive and unselected series of 93 necropsies and atheromatous ulceration was found in $6 \%$ of their cases. All these lesions, however, were located in the subclavian arteries. No ulceration of the wertebral arteries themselves was found. Haemorrhages in the base of the plaques similar to those described in the carotid sinuses were seldom seen in the vertebrobasilar arterial system. Imparato and co-workers (1981) in their study on cervical vertebral angioplasty wrote: "Microscopically, the plaques at the very origin of the vertebral were usually fibrous and smooth with no evidence of ulceration. This was similarly true of the subclavian plaque in the region of the vertebral and thyrocervival trunk origins. Intramural hemorrhage and ulceration were never seen. Friable atheromatous debris was occasionally found in the subclavian arterial wall'. In a roentgenologic study on 133 brachial angiograms of 71 patients, Kishore et al. (1971) found five ulcerative plaques in the innominate-subclavian-vertebral arterial 
system. Three ulcers were localized in the right subclavian artery proximal to the origin of the vertebral artery, while the other two were found in the cervical segment of the vertebral artery. This is approximately one-fourth of the incidence of ulceration they found in the carotid system in the same group of patients.

Thus the morphology of the complex atheromatous plaques in the carotid and vertebral arteries appears to differ in some important aspects. It is important to realize this when looking for a source of emboli responsible for transient or persistent episodes of vertebrobasillar ischaemia. These differences have made a definition of the aethiology of vertebrobasilar insufficiency difficult.

Recent work by Narotomi and associates (1979) showed that, in contrast to patients with transient ischaemic attacks of the carotid territory, patients with vertebrobasilar arterial insufficiency had significantly higher blood pressure than normal subjects. Moreover, in patients with vertebrobasilar arterial insufficiency cerebral blood flow passively followed the blood pressure levels, while during induced orthostatic hypotension flow in the brain stem-cerebellar region became significantly reduced. These results indicate impaired autoregulation in the brain stem-cerebellar region in patients with vertebrobasilar insufficiency.

\subsection{THE SIGNIFICANCE OF ATHEROSCLEROTIC DISEASE OF THE INNOMINATE-SUBCLAVIAN-VERTEBRAL SYSTEM AND ITS INTERACTION WITH DISEASE OF THE CAROTID SYSTEM}

Atherosclerotic lesions of the innominate and subclavian arteries may be a source of cerebral emboli. Obstructive disease of these vessels is also of interest in developing a subclavian steal syndrome. In most patients, however, this syndrome is asymptomatic. If there are symptoms of cerebral ischaemia, a subclavian steal syndrome is usually characterized by transient episodes of ischaemia in the vertebrobasilar territory. The subclavian steal syndrome will be discussed in more detail in chapter 5.4 .

Despite the high incidence of severe stenosis and occlusion at the origin of the vertebral artery from the subclavian arteries, strokes in the vertebrobasilar territory have only rarely been attributed to atherosclerosis at this site. In many patients there is a patent collateral circulation via the contralateral artery and symptoms of cerebral ischaemia will not occur. In the event of hypoplasia of one vertebral artery or in the event of the atherosclerotic process involving both vertebral arteries an extensive collateral circulation develops from the ascending cervical and deep cervical branches of the subclavian arteries and from the occipital branches of the external carotid arteries. As in subclavian steal syndromes, obstructive disease of the proximal part of the 
vertebral arteries produces transient ischaemic attacks but only few strokes (Fisher, 1970, Moufarry et al., 1984). Thus in contrast to the carotid territory unilateral of bilateral occlusion of the extracranial part of the vertebral arteries is apparently well tolerated.

Intracranial occlusion of the vertebral artery is a severer condition. This location often precludes the development of adequate collateral circulation and extension of the thrombosis into the lumen of the basilar artery or embolization of a loosely adherent clot may increase the neurological deficit. Unilateral intracranial occlusion of a vertebral artery of ten gives rise to the lateral medullary syndrome (Fisher et al., 1961). Bilataral occlusion of the vertebral artery in the intradural segment is a severe condition with an unfavorable prognosis (Caplan, 1983). In both conditions there may be a more or less prominent history of transient ischaemic attacks in the posterior circulation territory.

Moreover, it is important to emphasize here that in many patients with transient ischaemic attacks of the vertebrobasilar territory the extracranial cerebral vasculature is quite normal or atherosclerotic lesions are mainly located in the carotid arteries. Ueda and co-workers (1979) correlated the syndromes of transient ischaemic attacks of the carotid and vertebrobasilar arterial territories with the results as obtained with four-vessel arteriography. Fourty-one percent of their patients with vertebrobasilar transient ischaemic attacks had significant stenosis of the innominate, subclavian, or vertebral arteries. However, $31 \%$ of the patients of this group had atherosclerotic lesions only in the carotid artery and $28 \%$ had no demonstrable lesion at all. Fields and associates (1970) mentioned transient ischaemic attacks of the vertebrobasilar territory in 41 to $55 \%$ of their cases with lesions in the carotid arteries. Ziegler and co-worker (1973) found that $28 \%$ of the patients with only symptoms of cerebrovascular insufficiency of the posterior circulation showed a significant stenosis of the carotid artery. Most of these investigators, however, did not give detailed information about the arteriographic status of the innominate, subclavian, and vertebral arteries.

Probably the reason that the opinions on the therapeutic results of carotid endarterectomy in this group of patients vary so much lies in the fact that in patients with transient ischaemic attacks of the vertebrobasilar territory atherosclerotic lesions might be located in the carotid system, in the innominatesubclavian-vertebral arterial system or in both. Rosenthal and co-workers (1978) described in a long-term retrospective follow-up study that $80 \%$ of the patients with symptoms of vertebrobasilar insufficiency alone or with symptoms of vertebrobasilar insufficiency plus symptoms of transient or permanent neurological deficit of the carotid territory improved by carotid endarterectomy. The data in the reports of other investigators (Humphries et al., 1965, Ford et al., 1975) seems to support their findings. On the other hand, 
McNamara and colleagues (1977) and Owens and colleagues (1980) mentioned that the results of their study suggested that carotid endarterectomy has little or no therapeutic value in treating patients with cerebral ischaemia of the posterior circulation. In the joint cooperative study Fields and co-workers (1970) found an almost identical frequency of recurrent vertebrobasilar transient ischaemic attacks in patients treated by carotid endarterectomy and those who where treated nonsurgically. Their results indicated that carotid endarterectomy is of considerably less benefit in patients with nonhemispheric transient ischaemia than in those with hemispheric transient ischeamia and suggested that carotid endarterectomy did not alter the natural history of ischaemic disease of the vertebrobasilar territory.

The above mentioned studies make it clear that vertebrobasilar insufficiency is not a homogeneous entity. Instead it includes a variety of vascular pathologies at various locations, with diverse pathogenetic mechanisms, and with different clinical prognosis. For this reason it is not surprising that a review of the literature gives a significant difference in the probability of the occurrence of stroke in studies on patients with transient ischaemic attacks in the vertebrobasilar territory. Marshall (1964) and Ziegler and co-worker (1973), for example, noted a much better clinical outcome in patients with transient ischaemic attacks in the vertebrobasilar system than in patients with transient ischaemic attacks in the carotid system. On the other hand, Cartlidge et al., (1977), Jones et al., (1980), and Patrick et al., (1980) found no difference in stroke probability in these two subgroups. They suggested that transient ischaemic attacks in the vertebrobasilar arterial system have a grave prognostic significance of persistent neurological deficit and therefore need an adequate diagnosis and therapy. In a prospective study Heyman and coworkers (1984) determined the long-term outcome of transient cerebral ischaemia following medical and surgical treatment. The 5-year cumulative rate of completed stroke was $26.3 \%$ in patients with hemispherical ischaemia, compared to a rate of $22.1 \%$ in patients with vertebrobasilar ischaemia.

The presence of an atherosclerotic lesion in one proximal internal carotid artery makes it likely that a similar lesion is present in the contralateral internal carotid artery. However, the frequency of atheromatous lesions in the carotid system in the presence of atheroma of the vertebrobasilar system and vice versa is less well known. Hutchinson and Yates $(1956,1957,1961)$ were presumably the first who underlined the fact that atheromatous changes in a vertebral artery, often associated with atheroma in the carotid arteries, might play an integral role in certain syndromes previously attributed to occlusion of the carotid artery alone. Their necropsy studies indicated that stenosis or occlusion in the extracranial part of the vertebral artery may result in ischaemia and infarction of the cerebellum, brain stem and occipital lobes, partic- 
ulary when associated with disease of the carotid arteries. For this reason they introduced the term "carotico-vertebral stenosis".

Faris and co-workers (1963) subjected a group of 43 healthy male prisoners between the ages of 40 and 65 years to transbrachial percutaneous arteriography in order to visualize both vertebral and carotid arteries in the neck. Some kind of arterial lesion was demonstrated in $53.6 \%$ of the subjects and in ten cases more than one artery was affected. The abnormalities consisted of atherosclerotic plaques, complete arterial occlusion, arterial kinks, partial obstruction by arthritic spurs, extra-arterial fascial bands, or total absence of a vessel. The vessel most commonly involved was the right vertebral artery, then the right carotid, left vertebral and finally the left carotid arteries. The carotid arteries, however, were significantly more often affected by intrinsic, atherosclerotic processes than were the vertebral arteries. Comparison of this group of healthy men with a group of 68 male patients with cerebrovascular insufficiency in the same age group revealed only slight differences in radiological abnormalities.

In a study of 4,748 patients investigated by arteriography for cerebrovascular insufficiency, surgically accessible lesions of the extracranial parts of the vertebral arteries were commonly found, second in frequency to carotid bifurcation lesions (Hass et al., 1968). The left vertebral artery was involved slightly more often than the right. The incidence of lesions in the prevertebral segment of the vertebral artery being: stenosis-left $23.3 \%$, right $18.4 \%$; occlusion-left $5.7 \%$, right $4.0 \%$. The cervical and intradural segments of the vertebral artery were diseased less frequently and the frequency of stenosis or occlusion was practically the same on both sides. Stenosis of the proximal segment of the left subclavian artery was demonstrated in $12.4 \%$ of the arteries visualized, whereas the frequency was $4.2 \%$ in the innominate, and $8.3 \%$ in the proximal segment of the right subclavian artery. Complete occlusion of the proximal segment of the subclavian artery occurred three times more frequently on the left than on the right side and almost twice as often when the innominate and right subclavian arterial segments were considered to be one artery.

More recently Hennerici and associates (1981) showed that in patients with systemic atherosclerosis the incidence and extent of asymptomic extracranial arterial disease is high. In about a quarter of the patients with peripheral vascular and asymptomatic extracranial disease, the innominate-subclavian-vertebral system was involved in the atherosclerotic process. In a prospective study of neurologically asymptomatic patients with extracranial artery disease Hennerici and associates (1982) studied the natural history of atherosclerosis of the subclavian, carotid, and vertebral arteries. The degree of progression of extracranial artery disease in patients who developed a transient ischaemic attack or stroke did not differ greatly from that seen in 
patients who remained asymptomatic. However, symptomatic patients tended to show multivessel disease more frequently than asymptomatic patients. A combined lesion of the carotid and vertebral artery was the only significant indicator of increased risk of neurological deficit. It was six times greater than that for unitaleral of bilateral carotid lesions. This study, like others (Sindermann, 1967, Hennerici et al., 1980) indicates that involvement of the vertebrobasillar arterial system seems to be important in increasing the stroke risk of patients with obstructive lesions in the carotid system.

Although the relative figures of atherosclerotic disease of the carotid system, the vertebrobasilar system, and of combined carotico-wertebral stenosis by themselves differ from one study to another, most investigators agree that atherosclerosis diffusely involves the extracranial portions of the major vessels of the brain in most patients. However, in patients with stenosis or occlusion of the carotid system the pattern and degree of associated atherosclerosis in the vertebrobasilar system show wide variations and it should not be presumed that they will be of a similar degree of severity.

To summarize, the clinical outcome of any patient with atherosclerosis of the innominate-subclavian-vertebral system is dependent on:

1. the particular anatomy of that system. Developmental anomalies of the vertebral artery, for example, are very common and information about these variations in a given patient is important when judging the significance of atherosclerotic disease in this system.

2. the location and rapidity of the obstructive process. In general we may suppose that the more proximal the lesion is located the better the prognosis will be.

3. the avallability of adequate collateral circulation. Congenitally deficient or previously obstructed intracranial and extracranial vessels may diminish available collateral circulation.

4. the severity and extent of obstructive disease in the carotid system.

5. the physiological state of the systematic circulation and haematological system. 


\section{SOME SYNDROMES OF VERTEBROBASILAR ARTERIAL INSUFFICIENCY}

\subsection{INTRODUCTION}

Ischaemia of the vertebrobasilar territory is one of the most common manifestations of cerebrovascular disease and, at the same time, one of the most confusing. Its protean symptomatology and the variety of conditions with which it may be confused, makes diagnosis extremely difficult. Kubik and Adams (1.946) in their classic paper on thrombosis of the basilar artery were the first to describe the effects of occlusion of the basilar artery and showed that these effects might be recognized during life. At a later date, the different syndromes as a consequence of vertebrobasilar arterial insufficiency have been well defined as well as their correlations with the anatomy of the vertebrobasilar arterial system (Gillian, 1964, Caplan, 1981).

Transient ischeamic attacks were first described by Fisher in the carotid system, in 1951 , soon followed by reports of similar events occurring in the posterior circulation (Millikan et al., 1955). Although transient ischaemic attacks may occur in the territory of any of the cerebral arteries.there are good reasons for distinguishing between those occurring in the carotid tree and those which involve structures supplied by the vertebrobasilar arterial system. This distinction is not always simple, partly because the attacks seldom occur when a physician is available to examine the patient. Most frequently, data is based on information gathered from the patients or their families. As might be expected, this may be inaccurate because the family is usually excited and subjective, and the patients' alertness, insight, judgement, and memory may be impaired during the attack.

The frequency of transient ischaemic attacks in the vertebrobasilar arterial system is not precisely known. Recently in his textbook on cerebrovascular disorders Toole (1984) stated that 10 to $30 \%$ of transient ischaemic attacks involve the vertebrobasilar territory. The percentage will depend on the criteria used for classification, the populations surveyed, and is probably based on referral patterns wich preselect patients with carotid artery disease referred for surgical evaluation.

\subsection{TRANSIENT ISCHAEMIC ATTACKS IN THE VERTEBROBASILAR TERRITORY}

A transient ischaemic attack in the vertebrobasilar territory may be defined as a short-lasting and focal disturbance of cerebral function of the brain stem, cerebellum, or the temporal or occipital lobes arising as a result of ischaemia, occurring in a patient with degenerative cerebral vascular disease. 
The concept underlying the clinical term, transient ischaemic attack, is that of a period of ischaemia insufficient in duration and severity to cause infarction. A complete restoration of clinical function is essential to the definition. How long such a disturbance may last is entirely a matter of arbitrary decision. In most studies an upper limit of 24 hours has been set. The symptoms, however, will not always trouble the patient for this period of time. Instead, the residual evidence of the attacks, such as a trace of nystagmus or an equivocal plantar response, may be found during that period.

As in carotid artery disease transient ischaemic attacks in the vertebrobasilar arterial system need an accurate diagnosis and adequate therapy because approximately one third of the patiens with vertebrobasilar infarction have a history of a previous transient ischaemic attack (Jones et al., 1980, Patrick et al., 1980).

The part of the brain supplied by the vertebrobasilar arterial system contains anatomical structures subserving a multiplicity of functions. Hence disease within this system can lead to a myriad of symptoms and signs. The constellation of symptoms occurring in an individual case, however, usually clusters around one region of the brain. One patient for example, may have episodes of blindness with no vertigo, whereas another may have severe vertigo without any evidence of involvement of the occipital lobes. Usually symptoms, which result from a lack of function appear rapidly, reach a peak within seconds to a few minutes and last a few minutes or, occasionally, a few hours. The event may be singular or be repeated many times. Symptoms of transient reduction of flow in the vertebrobasilar territory are outlined below.

\section{vertigo \\ dizziness}

The vestibular neuronal complex is one of the structures in the brain stem which is most sensitive to ischaemia, probably due to the fact that they are supplied by long tenous vessels and are particulary vulnerable to a drop in pressure or flow. It must, however, be remembered that the labyrinth itself is supplied from the vertebrobasilar system as well. It receives its blood supply via the internal auditory artery which usually originates from the anterior inferior cerebellar artery, but may also arise directly from the basilar artery. Reduction of flow through this vessel may give rise to vertigo or dizziness with or without cochlear symptoms. Although vertigo and dizziness are the commonest symptoms encountered in vertebrobasilar transient ischaemic attacks they are particulary difficult symptoms to assess. Partly because they are subjective sensations of movement in space and it is difficult to know what the patient means when he complains of 'giddiness', 'dizziness', 'light headedness', 'unsteadiness' or other phrases. Strictly defined, vertigo refers 
to the illusory sensation of unidirectional movement in space. Many patients complain of a host of symptoms which they can only describe vaguely and unsatisfactorily. Before deciding that these symptoms are due to vertebrobasilar disease one should therefore look for additional signs of brain stem involvement. These symptoms per se are not adequate signs for diagnosing a vertebrobasilar transient ischaemic attack. In assessing associated symptoms and signs one has to realize that complaints such as nausea, vomiting, diarrhoea, sweating, faintness and an inability to stand may all be the result of the vertigo or dizziness itself. They cannot be used as a neccessary indication of a more extensive brain stem involvement. However, if one of the symptoms described below is an accompanying phenomenon it is likely that the brain stem is the site of the disturbance. In the event of a combination of vertigo or dizziness and a loss of vision the vascular origin of the disorder is clearly established, because it is only via their common blood supply that the brain stem and the occipital lobes can be linked.

According to many authors (Williams et al., 1962, Bradshaw et al., 1963, Paal, 1981) the vertigo is of the intermittent and rotary type and of central origin. An explanation for the different types of vertigo and dizziness as a result of peripheral, systemic or central causes of disease is beyond the scope of this chapter. In this respect we may refer to the papers recently published by Troost (1980), Patterson (1982), and Hülse (1982).

The belief, that vertigo and dizziness are more or less required symptoms before the diagnosis of transient vertebrobasilar insufficiency is possible, is incorrect because many of the symptoms described below may occur without them.

\section{tinnitus \\ deafness}

Whilst vertigo and dizziness are symptoms often encounterd in transient ischaemic attacks in the vertebrobasilar territory, tinnitus or transient deafness is probably most rare. In isolation these symptoms are uncommon in patients with atherosclerotic disease of the vertebrobasilar arterial system. They are more indicative of disease effecting small and intermediate arteries such as periarteritis nodosa, systemic lupus erythematosus, or lues.

\section{dysarthria}

dysphagia

Dysarthria of the bulbar or cerebellar type is a common phenomenon in vertebrobasilar transient ischaemic attacks. It may be confused with dysphasia and therefore lead to an incorrect diagnosis of transient ischaemia in the carotid artery territory. 
Dysphagia on the other hand is an uncommon symptom, presumably because the disturbance does not usually persist long enough for the patients to have the opportunity of testing his ability to swallow.

\section{ataxia}

Ataxia is another common symptom which is usually bilateral and affects posture and gait, more than functions which involve use of the arms. It is not always possible to decide whether the ataxia is due to a cerebellar disturbance or whether it is a concomitant of vertigo. Sometimes both components may in fact be present.

\section{hemiparesis \\ hemiplegia \\ hemiparaesthesias \\ pain}

Transient hemiparesis or hemiplegia may result from episodic focal ischaemia in the brain stem. When these signs alternate from side to side or when there is a bilateral involvement in one attack, they can easily be distinguished from similar symptoms caused by ischaemia in the cerebral hemispheres. Characteristically they involve the arm and leg but spare the face. Facial weakness is more commonly a symptom of transient ischaemic attacks in the carotid territory. When it is a sign of vertebrobasilar insufficiency, it is usually lower motor neuron in type and it may be unilateral or bilateral.

Similarly hemisensory disturbances may occur, consisting of pins and needles or numbness. Often they involve the face, are usually bilateral and commonly circumoral in distribution. In the case of numbness the patients may recognize it as being like the sensation he experienced when he had a nerve block for a dental treatment.

\section{blurred vision}

\section{photopsias}

\section{hemianopsia}

Episodes of bilateral blurring or dimming of vision occur almost as frequently as vertigo in patients who have vertebrobasilar insufficiency. These episodes may be abrupt in onset, giving rise to an almost immediate blackness, or they may develop over the course of a few seconds, greying of vision preceeding the loss. Total visual loss, however, is rare with vertebrobasilar transient ischaemic attacks. Often the symptoms are described as "the feeling that someone has turned down the lights', or as 'a sensation of looking 

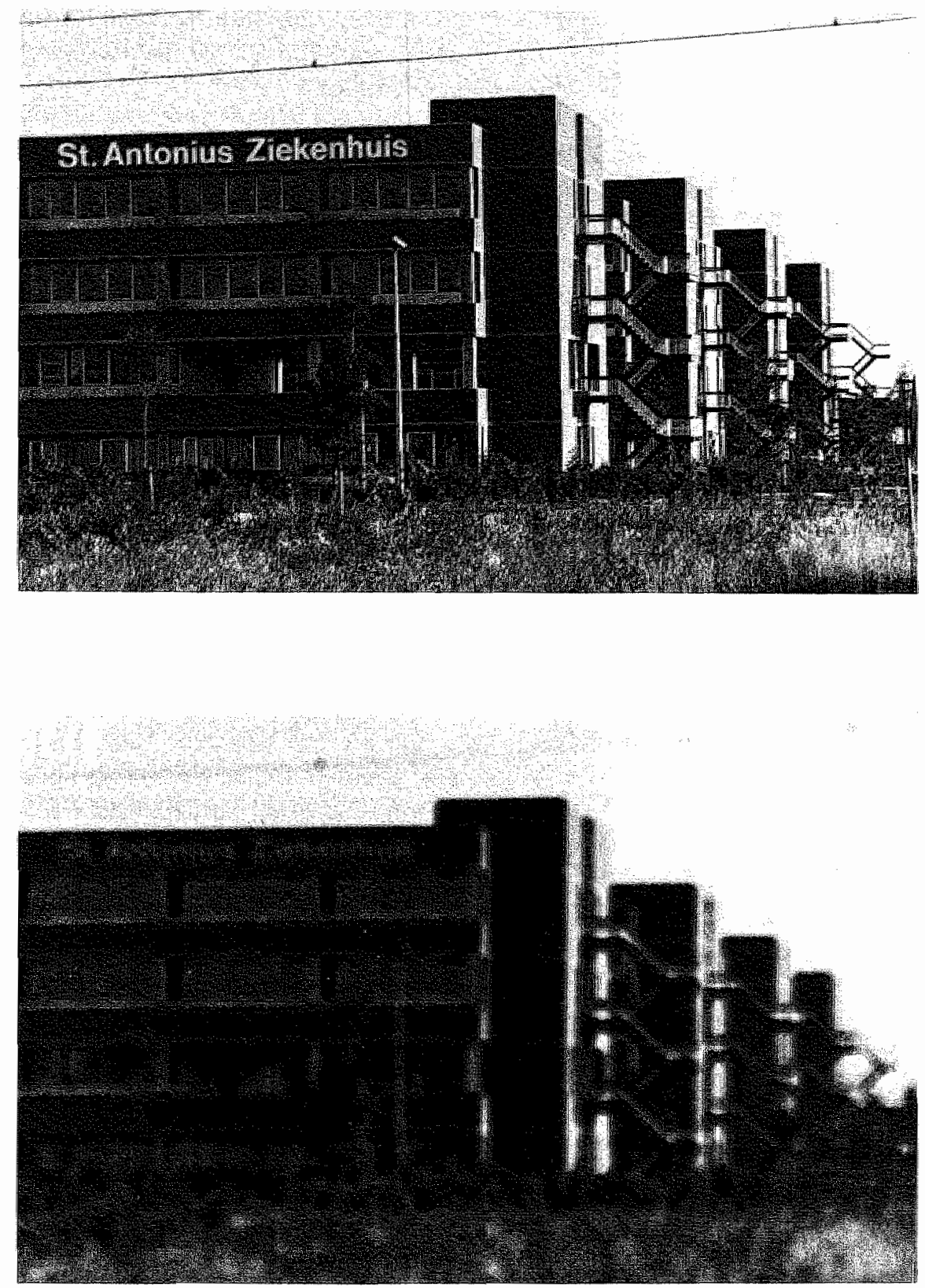

Figure $5.2 / 1$

Bilateral blurring of vision in vertebrobasilar arterial insufficiency. This is the front near the main entrance of the St. Antonius Hospital, Nieuwegein, The Netherlands. The blurred photograph simulates the momentary sensation described by the patient. 
through fog or smoke' (fig. $5.2 / 1$ ). The patient is usually distracted by these sensations, but seldom has time to evaluate his loss of visual field before normal sight returns. Associated sensations of colour are conspicuously absent. The episodes persist for only a few minutes, rarely more thans 3 or 4 , and restoration of vision is usually gradual, a period of blurred vision preceding total recovery. This type of disturbance depends upon a simultaneous reduction of flow through both posterior cerebral arteries.

Although photopsias are infrequent in occipital transient ischaemic episodes, attacks of longer duration may be accompanied by flickering, flashing scintillations in a homonymous field of vision. Occasionally an eldery patient without a history of migraine describes the sudden onset of silvery, zig-zag, shimmering sensations that begin near the right or left side of central fixation and then slowly move toward the periphery. It is not known in what way these episodes are related to the bilateral attacks of visual blurring described above. According to Wylie and co-worker (1970) it seems possible that these scintillations could be evoked by arrival of microemboli in the pial vascular network of the occipital pole.

Another manifestation which occurs when the posterior cerebral artery on only one side is involved is the development of a partial or complete contralateral, homonymous hemianopsia. If this symptom is simultaneously associated with a hemiplegia or hemiparesis on the same side a lesion in the carotid territory is more probable.

Yet another type of disturbance is the narrowing of the visual field from the periphery to the centre, with some degree of preservation of the central part. This is attributed to that part of the occipital cortex concerned with macular vision being anastomotically supplied via the middle cerebral artery.

\section{diplopia \\ oscillopsia \\ pollyopia}

Transient diplopia is a common symptom of vertebrobasilar insufficiency, but it is less frequent than blurred vision. It signifies a transient impairment of brain stem ocular motor control, such as internuclear paresis, skew deviation, or convergence weakness. The diplopia may be horizontal or vertical. Usually it persists for a few minutes, but occassionally it lasts for an hour or more (Hoyt, 1963). A transient weakness of the ocular muscles may produce nystagmus.

Some patients describe episodic 'jumping of their vision' (oscillopsia) during attacks of vertigo or dizziness.

Polyopia, seeing multiple images, is probably a cortical phenomenon and rarely seen in patients with vertebrobasillar insufficiency. More often it is an early symptom of cataract, due to irregular refraction by the lens. 
Drop attacks are a puzzling phenomenon, often attributed to vertebrobasilar ischaemia. The term is used in the sense of momentary falling without noticable loss of consciousness. The patient is suddenly, without warning or provocation, aware that he is falling or actually upon the ground and is usually able to rise immediately. He falls forward in flexion and often bruises his knees. There is no change in colour or any other somatic associated sign. Usually the attacks happen when standing still or walking. The patient itself is absolutely certain that he did not loose consciousness. However, the question as to whether consciousness is lost or not is a difficult one. We know that patients can loose consciousness momentarily without being aware of it, as occurs in petit mal. As a rule such brief interruptions of consciousness do not cause the patient to fall. Current theory attributes drop attacks to brief ischaemic episodes involving that part of the brain stem reticular formation which projects to the spinal motor neurones. The sudden loss in background tonus results in a general muscular hypotonus and in the patient falling to the ground. Though this is an attractive theory proof is lacking. According to some authors (Sheehan et al., 1960, Kubala et al., 1964) drop attacks are pathognomonic of transient ischaemic episodes in the vertebrobasilar territory. Many patients, however, may experience these attacks over months or years without ever developing other symptoms and signs of vertebrobasilar insufficiency. The occurrence of drop attacks alone does not permit a confident diagnosis of vertebrobasilar transient ischaemic attacks to be made.

\section{global amnesia confusion}

A transient ischaemic episode of the mediobasal aspect of the temporal lobe, the limbic system and the cranial part of the reticular formation, parts of the brain which are supplied by branches of the posterior cerebral artery, may give rise to the syndrome of transient global amnesia. In this syndrome the patient loses his memory of current events for a period of some hours. Orientation and behaviour appear completely normal. Subsequently, however, it becomes apparent that the patient has no reflection on what he was doing during the attack.

Another clinical picture, which contrasts markedly with that of global amnesia, is a confusional episode in which the patient becomes disorientated, especially regarding time and place. 


\section{unconsciousness}

\section{syncope}

Disturbances of consciousness as episodic confusion, loss of awareness or clouding of consciousness are sometimes described in patients with vertebrobasilar ischaemic attacks (Williams et al., 1962, Bradshaw et al., 1963). With the exception of the possible problem of consciousness during drop attacks, loss of consciousness is seldom seen in transient vertebrobasilar insufficiency.

The common phenomenon of syncope is of course the result of brain stem ischaemia so that to satisfy the purist it should really be included in the disorders under discussion. However, it is rarely a symptom of atherosclerotic disease of the vertebrobasilar arterial system. By far the most common cause is a dysfunction of the cardiovascular system for example orthostatic hypotension, cardiac arrythmia or other disorders of the conduction system of the heart.

\section{headache}

Headache is also a common symptom of transient episodes of vertebrobasilar insufficiency, and often persists for a longer period of time than the other symptoms, or even may occur independently (Bradshaw et al., 1963).

Abnormal visceral sensations such as distortion of taste, feelings of fear or unreality may in rare instances be experienced at the same time as other symptoms of vertebrobasilar insufficiency. They probably relate to ischaemia in the temporal lobes (Williams et al., 1962).

The diagnosis of transient ischaemic attack depends entirely upon a history of the patient's symptoms, except in those relatively few instances when a physician is present to observe the attack. For this reason, the skill with which the history is taken and interpretation of the history become of considerable importance. The diagnosis of transient ischaemic attack in the vertebrobasilar arterial system should be made not on the basis of a history of one of the above mentioned symptoms alone, but only when some of these symptoms are associated. Disturbances in sensation of the face and limbs and motor dysfunction, consisting of paralysis, weakness, or clumsiness of the limbs, are the most common symptoms (Heyman et al., 1984).

Usually the variety of clinical manifestations associated with transient ischaemic attacks in the vertebrobasilar arterial territory produce a more complex profile than that found in ischaemia in the territory of the carotid artery. Sometimes motor, sensory, or visual defects constituting vertebrobasilar transient ischaemic attacks may be unilateral, and in such instances the locus of ischaemia in either the carotid or the vertebrobasilar arterial territory may be difficult to determine. 


\subsection{THE SIGNIFICANCE OF DEGENERATIVE OSTEO-ARTHRITIC CHANGE OF THE CERVICAL VERTEBRAE IN VERTEBROBASILAR ARTERIAL INSUFFICIENCY}

Degenerative osteo-arthritic change of the cervical spine is a very prevalent condition. Many investigators (Hult, 1954, Gatzweiler, 1955, Süsse, 1955, Brain, 1963) have pointed out a steady increase in both the prevalence and the severity of this condition with age. After the age of 55, degenerative changes of the vertebral bodies, joints or discs are more often present than not. Although the incidence is approximately the same in men and women, women tend to be less severely affected than men (Brain, 1963). It should be borne in mind that in many patients the degenerative changes are harmless. Therefore, pathological findings in the sense of degenerative articular of disc lesions of the cervical spine should be regarded merely as potentially morbid.

Our knowledge of the degenerative changes of the cervical spine has steadily increased. First with regard to the effect of the disease upon the spinal cord and nerve roots. More recently concerning its possible influence upon the cerebral circulation through the vertebral arteries. As described in chapter 4 the most common aetiology of vertebrobasilar insufficiency is that of intraluminal atheroma producing decreased flow volume or emboli in the vertebrobasilar arterial system. However, when such symptoms as nausea, vertigo, ataxia, or drop attacks occur associated with head movements, the area of primary suspicion should be the cervical segment of the vertebral artery.

Unfortunately, the terminology used in the literature to describe the various degenerative changes in the vertebral column shows a regrettable lack of uniformity. Within the framework of this section we shall not enter into details. The suggested definitions are merely intended to clarify the anatomical considerations and pathogenesis of spondylosis of the cervical vertebral column.

The morphology of the articulations between the cervical vertebrae is of great importance. The lower five cervical vertebrae are connected together by three joints: the intervertebral disc anteriorly , the two zygopophyseal joints posteriorly, and, in addition to these, the neurocentral joints (fig. 5.3/1). The latter consist of a projection of bone from the posterolateral portion of the vertebral body articulating with the vertebra above. They lie between the disc and the nerve root canal.

Cervical spondylosis and all its manifestations are a product of cervical disc degeneration. It implies a mechanical breakdown of the integrity of a cervical disc which initially produces symptoms due to mechanical instability. Only in the later stages are bony outgrowths formed in relation to the articular processes. With the onset of disc degeneration, the intervertebral disc loses height. 


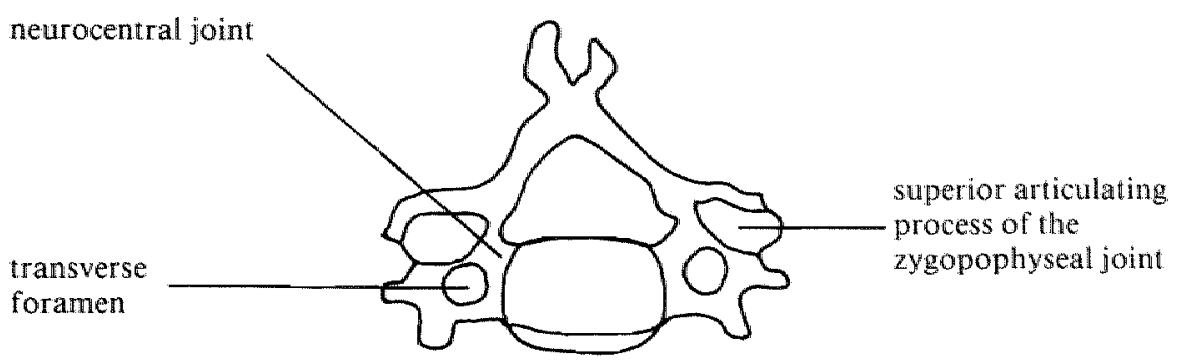

Figure 5.3/1

A superior view of the fifth cervical vertebra. It shows the close relationship of the vertebral artery to the neurocentral and zygopophyseal joints.

As it does so, the bony prominences formed by the neurocentral and posterior joints approach one another and eventually touch. With further disc degeneration the articular processes are firmly pressed together and osteophytic excrescences form. In the later stages of degenerative disc disease the bony outgrowths in relation to the neurocentral or posterior joints may encroach upon the nerve root canal. If an osteophyte, developing on the neurocentral joint, extends laterally or one on the posterior joint extends anteriorly, the vertebral artery foramen may be encroached upon and the vertebral artery may be compressed to a significant degree. Occasionally these osteophytes may produce severe kinking of the vertebral artery, resulting eventually in a stenosis or thrombosis that may extend distally.

Degenerative changes are not equally prevalent in all segments of the cervical column. The most pronounced changes occur in the middle and lower segments between the fourth and seventh cervical vertebral bodies (Krogdahl et al., 1940, Taylor, 1953, Tepe, 1956). In the unco-vertebral region degenerative changes have also been seen in higher segments (Krogdahl et al., 1940).

In their clinico-pathological study of the cervical segment of the vertebral artery, Hutchinson and Yates (1956) called attention to the fact that distortion of the vertebral artery was sometimes caused by bony changes in the spine from cervical spondylosis. They proposed the possibility that cervical spondylosis, common enough in the same age group as signs and symptoms of vertebrobasilar arterial insufficiency and capable of distorting the vertebral artery, might play a subsidiary role in obstructing one or other vertebral artery during movement of the cervical spine. In this respect it is of great interest that some investigators (de Kleyn et al., 1933, Tatlow et al., 1957) described a decrease of vertebral circulation on one side when the head was turned backwards and to the opposite side in postmortem material. They commented that there was no difficulty in stopping the circulation in one 
vertebral artery after hyperextension and rotation of the head to the opposite side. The reason that symptoms of vertebrobasilar insufficiency do not occur in normal individuals probably is that there is adequate circulation from the contralateral vertebral artery or the anterior circulation. In these studies the vertebral artery was usually compressed at the level of the atlanto-axial articulation. However, in one cadaver with cervical spondylosis, illustrated by Tatlow and co-worker (1957), there was significant worsening of the compression of the ipsilateral vertebral artery by an osteophyte during rotation of the neck. Virtamo and co-worker (1957) and Constantin and co-worker (1971) also demonstrated in cadlavers marked disortion and compression of the vertebral arteries by osteophytes, especially with the head turned to the opposite side.

In his study on vertebral arteriography by radial artery catheterization, Radner (1951) noted lateral displacement of the vertebral artery by cervical osteophytes in only two instances of 221 patients. However, in a roentgenologic study on 46 patjents with symptoms due to cerebrovascular disease or cervical spondylosis, Sheehan and colleagues (1960) could confirm by arteriography the compression of one or other vertebral artery by osteophytes in 26 cases. They considered the syndrome as a disease entity and proposed the term 'spondylotic vertebral artery compression'. After review of the vertebral arteriograms in the 26 patients in whom cervical spondylosis was cornfimed by roentgenographic examination of the cervical spine with accompanying compression of the vertebral arteries, certain features were considered to be characteristic of this condition. In severe cervical spondylosis the cervical segment of the vertebral artery had a strikingly tortuous appearance, with multiple sigmoid curves, the lateral concavities of which lay opposite an intervertebral space. Stenosis was frequent and occlusion rare at the point of maximal concavity. Compression of the vertebral arteries by osteophytes might increase on the side opposite to which the head was turned by stretching. However, compression was more severe on the same side due to pressure against the osteophytes. The most common site of vertebral artery displacement was between the fifth and sixth and the fourth and fifth cervical vertebrae. Less commonly, there was displacement between the third and fourth vertebrae. This rarely occurred between the second and third vertebrae.

At present the pathological changes of the cervical vertebrae and the signs and symptoms due to compression of the vertebral artery in cervical spondylosis are well established (Hardin et al. „1960, Hardin, 1962, Gray et al., 1971, Giroux, 1982). Many of the patients also have symptoms and signs referable to compression of the cervical spinal cord and nerve roots. The semeiology of spondylotic compression of the vertebral artery is episodic and is characterized by its reversibility in early stages, although repeated attacks tend to 
culminate in progressive irreversable damage to the brain stem (Sheehan et al., 1960). The signs and symptoms of this syndrome tend to be precipitated by hyperextension and rotation of the neck, and these manoeuvres may be used intentionally by the physician as a diagnostic test. Vertebral artery compression by osteophytes is frequently accompanied by atherosclerosis of the vertebral arteries or other segments of the cerebrovascular tree. This predisposes the patient to attacks of vertebrobasilar insufficiency because the collateral circulation may be reduced. Hypoplasia, artherosclerotic stenosis, or occlusion, and a subclavian steal syndrome of one vertebral artery will all predispose the patient to cerebral ischaemia when the opposite vertebral artery is compressed by osteophytes, especially if there is an incomplete circle of Willis.

In the living, compression of one or both vertebral arteries by osteophytes can be demonstrated by vertebral arteriography (Bauer et al., 1961). To date no reports are available about the use of non-invasive ultrasonic techniques for the investigation of the cervical segment of the vertebral artery in patients with cervical spondylosis. Our data of ultrasonic duplex scanning of the cervical segment of the vertebral artery as compared with the data of vertebral arteriography will be presented in section 9.4.4.

\subsection{THE SUBCLAVIAN STEAL SYNDROME}

Under physiological conditions, arterial pressure is higher in the aortic arch and arch vessels than in the intracranial arteries. As long as this normal pressure gradient is maintained, arterial blood ascends through the carotid and vertebral arteries to supply the intracranial structures. However, obstructions situated in strategic locations may invert these pressure gradients and cause blood to flow in the reverse direction from the head toward the heart and upper extremities.

This unique pathophysiologic situation in which blood is diverted from the brain and used to supply the upper extremities, was originally recognized and reported by the Italian surgeon Contorni in 1960. One year later Reivich and colleagues published a report of two patients in whom they observed a reversal of blood flow through the left vertebral artery. In both cases the anatomic lesion producing the reversal of blood flow was a stenosis of the left subclavian artery proximal to the origin of the vertebral artery. In the same issue of the journal that contained this article, there appeared an editorial about this particular vascular derangement by C.M. Fisher entitled 'A new vascular syndrome - The subclavian steal'. Since then numerous articles have appeared, reporting both the clinical and arteriographic descriptions of the phenomenon (e.g. Vollmar et al., 1965, Santschi et al., 1966, Fields, 1970, Fields et al., 1972). A similar condition of stealing blood from the cerebral circula- 
tion with occlusion of the innominate artery and reversal of blood flow in the right carotid and vertebral arteries has also been described (Ramirez-Boettner et al., 1966, Cala et al., 1972).

The subclavian steal syndrome consists of a stenosis or occlusion of the innominate or subclavian artery proximal to the origin of the vertebral artery. The obstructive lesion causes a reduction in pressure distally, with the result that blood, instead of coursing upwards in the corresponding vertebral or carotid artery to supply the brain, actually flows downwards in reverse fashion, siphoning or stealing blood from the cerebral circulation. Although the vertebral artery is, in the majority of cases, the main route of this siphoning effect, it has subsequently been shown that other arteries such as the internal and external carotid arteries or the thyrocervical and costocervical trunks, are also involved (Parrott, 1964, Bosniak, 1964-b, Vollmar et al., 1965).

Obstructions in different locations produce different forms of 'steals'. In all of them the volume and direction of abnormal flow depends on (1) the anatomy of the aortic arch and aortocranial arteries, which may be normal or anomalous; (2) the location of the obstructions; and (3) the balance between the demands of the limb and cerebral vascular beds for blood. In a particular patient, the anatomy and location of lesions remain the same, but the needs of the interconnected vascular beds constantly change. This causes the volume of reversed flow to fluctuate. For example, muscular exercise causes vasodilatation of the vascular beds of the limb and augments the siphoning effect. Symptoms of vascular insufficiency in the brain or upper extremity will occur only if the metabolic needs of these tissues exceed the amount of blood available to them. In patients with collateral channels able to provide the adequate nutrient requirement, extensive volumes of blood may be siphoned without producing cerebral vascular insufficiency. In other patients small volumes may exceed reserve and produce symptoms.

A significant blood pressure differential between the upper extremities and decreased or absent arterial pulsations in the arm involved are present in practically every patient. Usually, the systolic blood-pressure readings in the two arms show a difference of at least $20 \mathrm{mmHg}$, but diastolic pressures may remain equal. Auscultation over the subclavian or vertebral artery frequently reveals a systolic bruit, usually on the ipsilateral side but occasionally on the contralateral side, caused by altered haemodynamics. In some patients, exercising the arm involved increases the blood pressure difference, causes the ipsilateral radial pulse to disappear, accentuates the supraclavicular bruit, and induces claudication; all signs of increased demand for blood by the exercising muscles. Symptoms of vertebrobasilar insufficiency will only occur if the siphoning from the cerebral circulation can not be compensated by cerebral collateral pathways. Therefore, additional factors which influence 
the presence or absence of neurological symptoms include the adlequacy of collateral circulation and concomittant lesions of other extra- or intracranial arteries. As more than $80 \%$ of the patients with a subclavian steal syndrome show atherosclerotic disease involving one or more of the other arteries of the cerebrovascular tree (Santschi et al., 1966, Fields et al., 1972), it is understandable that the minimum degree of stenosis of the subclavian or innominate artery does not appear to be a fixed amount. Most of the patients have a tight stenosis or occlusion, but some show the typical syndrome with only a stenosis with a diameter reduction of $40 \%$ (Fields et al., 1972). Although all the signs and symptoms mentioned in section 5.2 can be seen in patients with a subclavian steal syndrome, the most common complaints of cerebral ischaemia are vertigo, dizziness, and bilateral visual blurring. Less common are ataxia, hemiparesis, tetraparesis, and hemiparaesthesias (Vollmar et al., 1965, Fields et al., 1972). Usually a subclavian steal syndrome is a harmless condition and needs no surgical treatment.

Reversal of flow in the vertebral artery can be demonstrated by contrast arteriography. As arteriography is an invasive technique, it can dramatically disturb the haemodynamics of the posterior circulation. Consequently, false negatives as well as false positive results are described. At present Doppler examination of the subclavian, axillary, brachial, and vertebral arteries is a standard measurement in the ultrasonic cerebrovascular evaluation of many laboratories. When using the hyperaemia test the accuracy of the diagnosis of a subclavian steal syndrome with transcutanuous Doppler techniques is very high (von Reutern et al., 1976, Berguer et al., 1980, Pourcelot, 1982). Moreover, ultrasonic recording of vertebral artery blood flow does not disturb the haemodynamics and therefore allows a distinction to be made between the different stages of subclavian steal (Pourcelot et al., 1975, von Reutern et al., 1978). Recently Walker and associates (1982) described the use of duplex pulsed Doppler to identify subclavian steal. The data of our study dealing with subclavian steal will be presented in chapter 9.4.7. 


\section{DIAGNOSTIC APPROACHES}

\subsection{ARTERIOGRAPHY}

Since the Portuguese neurologist Egas Moniz introduced radiological imaging of cerebral vessels with contrast media into clinical neurology in 1927 , cerebral angiography has made impressive progress. This progress is linked to an improved knowledge of the radioanatomy of the cerebral vasculature, as well as to the introduction of the catheter method with selective angiography, the availability of safer contrast media and to the introduction of digital subtraction angiography.

The evolution of vertebral angiography was described extensively by Schechter and colleagues in 1973 . The desirability of a safe routine procedure and the difficulties in elaborating such a procedure are demonstrated by the large number of methods proposed for vertebral arteriography. In 1933 Moniz and co-workers were the first to introduce vertebral arteriography. The subclavian artery was exposed in the supraclavicular fossa, and the contrast medium was injected into this artery at a point distal to the origin of the vertebral artery. Since that time, many direct and indirect methods have been described. Most of these techniques have been especially developed to visualize the intracranial branches of the posterior circulation as for example in the event of a cerebral tumor or a saccular aneurysm of the basilar artery. These direct techniques are of little value in diagnosing atherosclerotic disease of the vertebrobasilar arterial system because it is not possible to visualize the origin of the vertebral artery from the subclavian artery by a direct method. Ar additional disadvantage of these techniques is the risk of introducing vascular spasm. Apart from that, the danger of a mechanical lesion of the nerve roots of the cervico-brachial plexus, a Brown-Sequard syndrome caused by intradural injection of the contrast medium, and the formation of arteriovenous fistulae is always present.

In the last decade selective catheterisation of the branches of the aortic arch has been developed. While elaborating a method of thoracic aortography in post mortem studies Radner and co-workers (1951) often observed that the tip of the catheter deviated into the vertebral artery. The idea then arose, that in living subjects it might be possible to guide a catheter into the vertebral artery and to perform vertebral arteriography by the injection of contrast medium through the catheter. Radner (1951) extensively described this method in a study on 221 cases. The catheter was introduced under fluroscopic control and when it entered the vertebral artery $35 \%$ Diodrast was injected and the angiograms were made. The technique of vertebrobasilar arteriography by catheterisation was subsequently used and refined by such investigators as Olsson (1953), Hauge (1954), wan den Berg et al., (1963), Scatliff et al., (1965), Takahashi et al., (1969), and Peeters, (1969). 


\subsubsection{COMPLICATIONS OF VERTEBRAL ARTERIOGRAPHY}

Cerebral arteriography is a potentially hazardous procedure and should be performed only when strongly indicated. Nevertheless, it remains the major diagnostic tool in evaluating symptomatic patients with extracranial obstructive disease and is also used as a reference standard for evaluating newer noninvasive diagnostic modalities. The indications and contraindications for cerebral angiography as well as the frequency and reasons of complications are reviewed in detail in textbooks of cerebral angiography as for example by Newton and co-workers (1974) and Krayenbühl and co-workers (1982). Here only a brief survey of generally accepted ideas about predisposing factors, and particularly about the complications of vertebral arteriography will be given.

Owing to modern techniques and contrast media, the frequency of complications has decreased over the years. The published statistical data usually does not reveal how much experience the individual examiner has. It does show, however, that the complication rate is lower when the number of cases, and presumably the experience of the angiographer, is greater (Hessel et al., 1981). Mani and colleagues (1978-a) made an analysis of 5,000 catheter cerebrall arteriograms in two training and two nontraining hospitals. The level of experience of angiographers varied considerably between supervised first and second year radiology residents and fully trained neuroradiographers. The complication rate for the training hospitals (3.9\%) was significantly higher than that for the nontraining hospitals $(0.9 \%)$. However, the rates of permanent complication were identical $(0.1 \%)$. Therefore, the difference between the hospital groups may be explained by the number of transient complications. On the other hand, Olivecrona (1977) and Earnest and colleagues (1984) found no statistically significant correlation between the length of procedure and neurologic complications.

Furthermore, the underlying disease plays an important role in the occurrence of complications. The patient who has a disorder associaced with reduced perfusion of the brain is at greater risk of suffering a neurologic complication at cerebral arteriography than the patient with normal cerebral blood flow. Thus patients with atherosclerotic disease involving the extracranial or intracranial vessels, cerebral emboli or thrombosis, low cardiac output or decreased oxygen-carrying capacity are more liable to complications.

The risk of cerebral arteriography is increased in the elderly patient, especially in those which have a systematic vascular disease (Faught et al., 1979, Earnest et al., 1984). Hypotension, which reduces cerebral perfusion, is particularly dangerous in this group of patients. The exact mechanism by which complications occur in a state of reduced perfusion of the brain is uncertain. Various possibilities have been considered: hypoxia could potentiate the toxic 
effect of the contrast medium; transient displacement of the blood by the contrast agent could reduce oxygenation below a critical level; vascular spasm could be induced or aggravated by the contrast medium; the high osmolarity of the contrast could cause aggregation of the formed elements in the blood and increase blood viscosity.

Although the complication rate is somewhat higher in vertebral than in carotid arteriography, whether direct puncture or catheterisation is used, most studies show no striking statistical difference in complication rates between carotid and vertebral angiographies. Wishart (1971) carried out a thorough study on 447 catheterisations of the vertebral and carotid arteries. There was no remarkable difference between total complications rates in the two procedures. However, neurological complications were seen in $6.5 \%$ of the vertebral arteriographies, and in only $4.2 \%$ of the investigations on the carotid arteries. Mani and co-workers (1978-b) also failed to find statistically significant differences in neurological and systemic complication rates between patients undergoing vertebral injections or aortic arch injections, alone or in combination with carotid artery injections.

In the early years of vertebral arteriography many authors mentioned that the injection of the contrast medium provoked a more or less intense pain in the occipital region and nausea and vomiting were frequently seen in this procedure. Temporary complications as unconsciousness, unilateral or bilateral extremity weakness, blurring of vision and homonymous hemianopsia were described by most investigators in a small percentage of the examinations. These symptoms were attributed to the toxic effect of contrast material on the brain parenchyma and were probably due to a disturbance in the function of the blood-brain-barrier after the injection of the contrast medium (Radner, 1951).

A serious technical problem of catheterisation, which also can produce discomfort in the patient, is plugging of the vertebral artery at its orifice by the catheter. In such cases the rate of contrast material flow through the vertebral artery may be significantly decreased. Several authors have commented on the development of vertebral artery spasm during catheter arteriography of this vessel. There can be no doubt, that this does occur, particularly after prolonged attempt at catheterisation. It appears, however, that in most cases in which the flow of the medium has been delayed or stopped in the more distal segments of the vertebral artery, catheter plugging of the artery is involved. Checking for a normal washout of the contrast medium during the examination is particularly important with selective vertebral arteriography, in which the diameter of the catheter relatively closely approximates the diameter of the vertebral artery (Howland et al., 1964, Howieson et al., 1968). Ordinarily, the anglographer will anticipate this problem by selecting the larger of the two vertebral arteries for the investigation. 


\subsubsection{ACCURACY OF CEREBRAL ARTERIOGRAPHY}

Physicians have recognized that arteriography is primarily a morphological study. Arteriographic visualization of the arteries includes the pattern of arterial supply to organs or specific anatomic locations, the course of major branch channels and collaterals and the anatomic detail of the circulation in a particular area of interest. In many instances an identification of these morphological details is the only information demanded of this investigation. In some clinical circumstances, as for example, in atherosclerotic disease of the innominate-subclavian- vertrebral arterial system, a prerequisite to accurate diagnosis and treatment involves the identification of lesions which produce symptoms by virtue of their effect on pressure and flow. In these circumstances, the reduction in the cross-sectional area of the vessel is first estimated and with this estimate an assessment is then made of the effect of this change on pressure and flow. Finally, in some vessels such as the carotid artery, more detailed information regarding the surface characteristics of plaques is of interest.

It should be realized that in these instances a great deal is demancled of the arteriography and it is not altogether unexpected that the potential for error is considerable. While many of the problems are related to factors associated with interpretation, it is not uncommon for technical deficiences in the study itself to magnify these problems. It is therefore somewhat surprising that in spite of many years of extensive application in clinical practice and research studies, a critical evaluation of the accuracy of arteriography has only been performed in recent years.

In broad terms, evaluation of the accuracy of arteriography can be divided into three different groups. The first group includes all the studies which attempt to relate arteriographic findings to pathologic studies, either from surgical specimens or at autopsy. The second group includes studies in which functional changes are assessed on the basis of arteriographic changes and compared with those measured by physiologic testing procedures. Finally, in the third group intra- and interobserver agreement is judged when subjective interpretation of arteriograms is used. In view of our study of ultrasonic duplex scanning of the innominate, subclavian and vertebral arteries this last group is the most interesting.

It is essential that the arteriographer is aware of the tendency of atherosclerosis to develop at major branch points and bifurcations in the arterial tree. In clinical neurology this is critical in the arteriographic evaluation of the carotid and vertebrobasilar system as well. Adequate visualization of the innominate, subclavian, and vertebral arteries demands that exposures be obtained in projection planes which clearly outline the origin of these vessels. 
Especially for the vertebral artery this frequently requires multiple oblique projections. Not only are multiplane projections essential for evaluating the origin of the vertebral artery from the subclavian artery, but in the straight cervical segment of the vertebral artery, atherosclerosis tends to develop eccentrically and in relation to osteo-arthritic deformation of the cervical vertebrae. When this occurs the artery may appear normal in the lateral projection and only anteroposterior or oblique views will permit an appreciation of the extent of involvement.

Secondly an optimal visualization of atherosclerosis often requires that injection of contrast is performed as close as possible to the area under investigation to ensure that adequate dye concentrations are reached in the region of interest. For the arteriographic evaluation of the posterior circulation this demands views of the aortic arch as well as selective injections of the innominate, subclavian and sometimes even of the vertebral arteries. To detect lesions of the vertebral arteries, especially of the ostia and proximal parts, careful investigation with selective injection of the subclavian arteries and the use of cineroentgenography is imperative. This is caused by the complex anatomy of the vertebral arteries. On both sides the ostium and the very first proximal segment of the vertebral artery are generally located on the dorsal side of the subclavian artery just proximal to its most cranial part. Usually the best exposures of the proximal part of the vertebral arteries will be realized with oblique projections with the central ray angled in a craniocaudal direction. Often projections adjusted to individual anatomic variations are necessary. In a series of 100 cases Ludwig and his colleagues (1984) showed that visualization of atherosclerotic lesions at the ostium or in the proximal part of the vertebral artery was more than 3.5 times better when they administered subclavian artery injection in conjunction with video-recording compared with aortic arch injection only.

While it is recognized that atherosclerotic disease of the vertebrobasilar arterial system may be responsible for symptoms of cerebral ischaemia in many patients, a critical analysis with regard to the accuracy of arteriographic definition of atherosclerotic lesions in this part of the cerebral vascular tree is lacking. The carotid bifurcation is the only area of the entire extracerebral and intracerebral circulation which has been subjected to such an analysis. Without doubt this can be explained by the clinical importance of carotid artery disease in producing transient cerebral ischaemic attacks and stroke, and by the development of carotid endarterectomy.

With the recognition of the importance of emboli from uicerated plaques in the carotid bifurcation several investigators have attempted to define the accuracy of arteriography for the detection of ulcerated lesions. Most of these studies support the view that even with optimal imaging of the carotid bifur- 
cation, the accuracy of predicting the presence of an ulcerated plaque is probably not much greater than chance (Edwards et al., 1979, Thiele et al., 1983, Eikelboom et al., 1983). At present no data is available on the accuracy of arteriography in the detection of ulcerated lesions of the innominate, subclavian, and vertebral arteries.

The major stimulus responsible for increased interest in the accuracy of arteriography has been the emergence of alternative techniques for the detection of arterial disease. The dewelopment of noninvasive methods has required an evaluation of their accuracy by some independent standard, and as arteriography was so commonly used and accepted as the standard, all other methods have been evaluated against it. It is this aspect of arteriography which is being reviewed increasingly more critically with the recognition that the interpretation of direct morphological information may differ from one observer to another and even between the same observer on different occasions. This inter- and intraobserver variability of interpretation largely originates from the fact that under most circumstances qualitative information is assessed. Recognition of this fact has prompted the development of new approaches which attempt to derive quantitative information as a means of reducing this variability. While this approach has been investigated extensively in coronary arteriography (Zir et al., 1976, Brown et al., 1977), and aortography (Bruins Slot et al., 1981) it has not been widely employed in arteriographic studies of the cerebral vascular tree.

Chikos and co-workers (1983) determined in a prospective manner the inter- and intraobserver variability in measuring the amount of diameter reduction produced by plaques of the carotid bifurcation. They concluded that trained observers have an acceptable rate of agreement in estimating percent stenosis in the extracranial carotid system. In his study of the carotid bifurcation, de Vries (1984) compared the results of conventional arteriography with the results of intravenous digital subtraction angiography. In order to compare both methods and to determine the inter- and intraobserver agreement he used values of Kappa, a measure of agreement for categorical data in a two-way table, corrected for chance. Hardly any difference was found between both methods. Only the standard deviation of the mean calculated stenosis was somewhat smaller in conventional arteriography than in intravenous digital subtraction angiograhpy.

Because of our interest in diagnostic procedures which avoids the potential morbidity associated with arterial puncture, we compared (Eikelboom et al., 1983) intravenous digital subtraction angiography and ultrasonic duplex scanning with conventional arteriography with regard to their accuracy in quantifying internal carotid artery disease. The overall agreement corrected for chance (Kappa) for intravenous digital subtraction angiography was 0.738 \pm (SE) 0.049 . 
These studies show that although intravenous arteriography has limited resolution capabilities, it of fers the same possibilities as conventional arteriography with regard to its accuracy in quantifying atherosclerotic disease of the carotid bifurcation. An assesment of the accuracy of arteriographic interpretation of atherosclerotic lesions in the vertebrobasilar arterial system has not been properly performed. Recently Hesselink and co-workers (1984) described their results with intravenous digital subtraction angiography in a study on 111 patients with vertebrobasilar ischaemia. Although, in their opinion, $90 \%$ of the vertebral images were of diagnostic quality, the cervical vertebral arteries were not routinely included on vertebral arteriograms and, therefore, accurate comparison could not be made for the vertebral artery. In our experience intravenous digital subtraction angiography is inferior to conventional arteriography in obtaining high quality images of the ostia and first parts of the vertebral arteries.

\subsection{NON-INVASIVE TESTS}

Despite the success of real-time pulse-echo and Doppler techniques in the diagnosis of atherosclerotic disease at the carotid bifurcation and the excess of publications that these have spawned, only few attempts seem to have been made to apply the same techniques to the branches of the aortic arch and the vertebral arteries. The reasons for difficulty and relative neglect of the investigation of atherosclerotic disease of these vessels include the following: (1) This part of the cerebral vasculature has a relatively deep course and is overlaid by other arteries with which it may be confused. (2) The vertebral arteries in their bony canal are rather inaccessible, are commonly asymmetrical in size, and, more often than the carotids, subject to anomalous origin. (3) The haemodynamics of obstructive lesions on the innominate-subclavian-vertebral system are more complicated and the non-invasive techniques have largely been directed toward the detection of reversed flow in the vertebral arteries in steal syndromes. (4) Because of the lesser accessibility of the vertebral arteries, radical surgical treatment has largely been restricted to a few centres.

Franceschi and co-workers (1981) probably were the first to demonstrate that with real time B-mode systems it is possible to display the arch vessels and the vertebral arteries in the neck. Their photomosaic was made by the superimposition of nine separate scans made at different angles. It included the right carotid and vertebral arteries in the neck as well as the right subclavian and innominate arteries with their intrathoracic origin from the aortic arch.

All other attempts to investigate the innominate-subclavian-vertebral arte- 
rial system have been confined to the use of Doppler techniques and these can be broadly divided into those that produce a two-dimensional spatial display of the vasculature and those that do not.

\subsubsection{NON-IMAGING DOPPLER TECHNIQUES}

The examination of the innominate-subclavian-vertebral arterial system with non-imaging techniques is hardly ever performed alone but is usually carried out in addition to the examination of the carotid system. As with the examination of the carotid arteries, the vessel of interest is directly interrogated by holding the Doppler probe over the artery and orientating it until the audio-Doppler signal is obtained and maximized. Much of the skill of the examination consists of listening to this audio signal and trying to determine from its characteristics, as well as the positioning of the probe, whether it does in fact arise from the vessel of interest. Moreover, the examination of the vertebral artery suffers from two main restrictions. Firstly, a large part of the artery is inaccessible to ultrasound, being obscured by bone in its cervical segment. Secondly, the presence of numerous other vessels, especially in the lower neck, makes it difficult to be sure that the Doppler signal detected does in fact arise from the vertebral artery. Concerning the first of these difficulties, most workers have largely confined their efforts to examining the artery either low in the neck, before it enters its bony canal, or just below the base of the skull as it winds around the lateral mass of the atlas. On the other hand, Keller and co-workers (1976) developed a transoral approach with the transducer of a directional Doppler flow meter in the anaesthetized lateral wall of the pharynx. Because the spatial relationship between the probe and the vessel axis is known, the flow direction can be used as an additional diagnostic measurement. However, patient discomfort and swallowing artifacts have not made this a popular procedure. In order to overcome the second difficulty, that of distinguishing the vertebal artery from neighbouring or overlying vessels, many authors pointed out that such a distinction could be made from systemic arteries that supply high-resistant vasculatures by the fact that the vertebral artery has significant antegrade flow during diastole, because it supplies the low-resistance vasculature of the brain. However, in the case of a marked hypoplasia or tight stenosis in the intradural segment of the vertebral artery this will be a misleading characteristic. Moreover, the distinction of the vertebral artery from the internal carotid artery or branches of an external carotid artery can be improved when recordings are made only from arteries in which flow persists during compression of the common carotid artery on the same side.

The investigation of the proximal, prevertebral segment of the vertebral artery is possible (Barnes et al., 1975, Hennerici et al., 1981, Ringelstein, 
1984) if the overlying common carotid artery is manually displaced medially. The procedure has the obvious advantage that the signals are recorded from the vicinity of possible stenoses, since most stenoses occur at the origin of the vertebrall artery from the subclavian artery. Corson and co-workers (1977) described an alternative technique for the non-invasive study of the vertebral arteries. They placed the probe of a directional Doppler flow meter over the Chassaignac tubercle, which is the well-dleveloped anterior tubercle of the transverse process of the sixth cervical vertebra. The probe is then directed medially, inferiorly, and posteriorly, and the vertebral artery is found just before it enters its bony canal.

Probably all these techniques are difficult because only a few authors have described successful results. The most popular approach for the examination of the vertebral artery with non-imaging Doppler techniques is in its atlantic segment, where it curves around the lateral mass of the atlas (Pourcelot, 1975, von Reutern et al., 1976, Kaneda et al., 1977, De Bray et al., 1978, Mol, 1973, 1982, Pfeiffer et al., 1984). The probe is positioned behind the mastoid and directed medially, toward the opposite eyeball. From this position, however, it is often difficult to determine whether the afferent or efferent section of the atlantic segment of the vertebral artery is insonated by the ultrasonic beam. Therefore, it is not possible to determine the direction of flow with certainty by only using the Doppler sonographic curves. This can be done by functional tests, as for example, postischaemic hyperaemia produced by inflating a blood pressure cuff on the upper arm above systolic pressure for two minutes, and releasing the cuff rapidly.

All the non-imaging Doppler techniques described above used continouswave devices and thus have no range resolution. White and co-workers (1980) developed a pulsed Doppler system with a single channel that could be varied in both duration and range. Recordings were first made from the common carotid arteries. Without moving the probe the gate was then advanced by a time corresponding to a depth of one centimeter, which corresponds to the depth of the vertebral artery in its bony canal. The audio Doppler signal of the carotid artery disappears during this procedure and another arterial signal may be heard. To prove that this signal in fact originates from the vertebral artery the probe is then rotated around the trandsducer face in the long axis of the neck in a cranial and caudal direction. If the signal disappears or reappears after rotation moves the beam above or below the obscuring transverse process of a cervical vertebra, the operator can be reasonably sure that the signal does originate from the vertebral artery. Although the authors decided that recording vertebral artery flow by this technique was usually a simple and easily learned procedure, it was not always possible to ensure that the signals were originated from the vertebral artery. Sometimes the recordings were inadequate and there were times when they must have mistakenly recorded signals from other arteries. 
Many non-imaging Doppler techniques are of substantial help in making an estimation of the severity of the disease of the innominate-subclavianvertebral arterial system, but they do not as yet yield quantitative information. With the exception of reversed or oscillating flow in the wertebral artery in cases of subclavian or innominate steal, to date no reports are available about the accuracy of non-imaging Doppler techniques for the detection of obstructive lesions in the innominate and subclavian arteries. A subclavian steal syndrome can be reliably diagnosed by these techniques, but a differentation of the underlying vascular disease is not possible. It is also difficult to ascertain the accuracy of the various non-imaging Doppler techniques for the examination of the vertebral artery, although several authors (von Reutern et al., 1976, Keller et al., 1976, Kaneda et al., 1977, De Bray et al., 1978, White et al., 1980, Pfeiffer et al., 1984, Ringelstein, 1984) have reported their results in cases subsequently examined by angiography. The main problems are the investigation of the proximal, prevertebral segment of the vertebral artery and the differentiaton of severe stenosis, occlusion, hypoplasia, and aplasia. The only abnormalities that can be identified are: (1) Absent flow due to either an occluded or to an absent artery, (2) Asymmetrical flow either due to hypoplasia or to a severe stenosis proximal or distal to the site of the examination. (3) High-frequency signals caused by turbulence due to a stenosis near the site of examination. Also, some reports show that in a significant number of cases recordings must have been made from other arteries under the impression that the vertebral arteries were being interrogated. For these reasons it is understandable that Pourcelot (1982) states that the diagnosis of stenosis of the origin of the vertebral artery "is still difficult and the degree of accuracy needs to be improved in the future".

\subsubsection{IMAGING DOPPLER TECHNIQUES}

Since it is obvious that most of the difficulties in the Doppler examination of the vertebral arteries arise from uncertainty regarding the artery from which recordings are being taken, it is to be expected that Doppler scanning systems with spatial displays would be much more successful in examining the vertebral arteries. Spencer and co-workers $(1977,1981)$ appear to have made the first spatial images of the vertebral arteries. They scanned the artery with a continuous-wave imaging system and were able to display the prevertebral segment of the artery over a short distance. However, they do not state how frequently and reliably such images could be made as compared with angiography. White and associates (1978) first made spatial displays of the vertebral artery in its midcervical course. Such displays are so characteristic with their unique "beaded" appearance, that there can be no doubt of the identity of the vertebral artery. Their experience, however, showed that this 
technique was difficult and that satisfactory scans could only be made in 25 $40 \%$ of the patients examined. A second deficiency of this technique resulted from the fact that the vertebral arteries are only displayed in segments of their course between the transwerse processes of the vertebrae in the midcervical region, while stenoses occur near the origin of the vertebral artery from the subclavian artery. Subsequently, they adopted the technique originally described by Spencer and co-workers. In this way they were able to image the origin of the vertebral arteries, but once again the technique was difficult and it was impossible to complete a scan succesfully in a significant number of patients.

Wood and Meire $(1980,1983)$ described a 30 channel pulsed Doppler directional system with which they made images of the arch vessels and yertebral arteries. The relationship of the vertebral artery to the subclavian and common carotid arteries on the map confirmed its identity. Additional important information that the vessel is indeed the vertebral artery was gained from observing the characteristic velocity wave form pattern obtained from it. This new technique should impove the ultrasound diagnosis of occlusions or stenoses of the proximal vertebral artery. They only could compare the results of their technique with those of contrast arteriography for 18 vertebral arteries of 12 patients. Although these numbers are too small for a reliable statistical analysis, they give an indication of the accuracy of the technique in detecting the patency or occlusion of the vertebral arteries. The specificity in detecting a patent vessel is 15 out of 16 true negatives $(93.75 \%)$. The sensitivity in detecting the abnormal situation of an occluded vertebral artery is 1 out of two true negatives $(50 \%)$. No data is provided on the accuracy of the detection of a stenosis of the innominate and subclavian arteries, or at the site of the ostium of the vertebral arteries. Their major problem seems to be the visualization of the origin of the vertebral arteries, due to heavy calcifications within a localized plaque, or to a posterior angulation of the first few centimeters of the vertebral artery behind the subclavian artery. Furthermofe the technique is rather time consuming and required a high degree of hand-eyeear coordination from the technician.

\subsubsection{THE DUPLEX CONCEPT}

The duplex scanner consists of a real-time ultrasonic scanner and a pulsed Doppler flow meter to examine blood flow characteristics at a precise location in the blood vessel. It combines the advantages of both techniques used independently. The ultrasonic image of the arteries is an anatomical guide by which one is informed of the exact location and course of the vessels of interest. Moreover, in many cases the B-mode image provides useful information about atheromatous deposits within and along diseased arteries. 
The image can be stored, and one of the transducers is used as a gated pulsed Doppler transducer to sample blood velocity at a particular point in the blood vessel. The position of the Doppler beam is indicated as a white line on the display screen and the position of the sample volume is shown as a white spot on this line. The direction of the Doppler ultrasound beam and the position of the sample volume are adjusted using an arm which is connected by means of a servomechanism to the transducer. In addition to audible interpretation, real-time spectral analysis of the Doppler signals is used to evaluate the degree of stensosis. Video tape recordings can be made of both the B-scan display and the Doppler signals as well as a hard copy on lightsensitive paper to display the frequencies, the directional characteristics and the amplitude in shades of grey of the Doppler signal.

Ultrasonic duplex scanning with spectral analysis of the Doppler signal is a very reliable technique for the detection of atherosclerosis of the internal and external carotid arteries (Fell et al., 1981, Breslau, 1982, Ackerstaff et al., 1982). To date, no reports are available about the accuracy of this technique for the investigation of the innominate, subclavian, and wertebral arteries. 

PART 2. OWN INVESTIGATIONS

In ultrasonic imaging there are still some things we have yet to imagine. 



\section{EQUIPMENT, INSTRUMENTS, AND DATA ANALYSIS OF THE DOPPLER SIGNALS}

\subsection{THE INSTRUMENTATION}

A block diagram of our system is shown in figure 7.1/1. The basic elements of the ATL Mark V duplex scanner include: the scan head assembly, a $600 \mathrm{~B}$ pulsed echo system, a $400 \mathrm{~B}$ pulsed Doppler system, and a $851 \mathrm{~B}$ real-time digital scan controller. The associated display and recording instrumentation consists of a frequency multiplexer, a Nicolet spectrum analyzer (UA-500A), a Tektronix $5130 \mathrm{~N}$ oscilloscope, a Honeywell Visicorder oscillograph, and a Sony Umatic video cassette recorder (VO-2630).

The duplex scanner employs three 5- $\mathrm{MHz}$ ultrasonic transducers mounted on a rotating drum encased within a water-filled silastic boot. These transducers generate a two-dimensional image of the vessels of interest with 16 levels of grey. During the examination this image is displayed on an oscilloscope screen. The image rate is 30 frames per second and the sector angle of the Bmode image can be chosen at 45 or 90 degrees. We have routinely used a 90 degrees sector angle. The information is digitized and the B-mode image can be 'frozen'. In this mode one of the crystals mounted on the rotating drum is used as a single gate $5-\mathrm{MHz}$ pulsed Doppler with a quadrature output. The

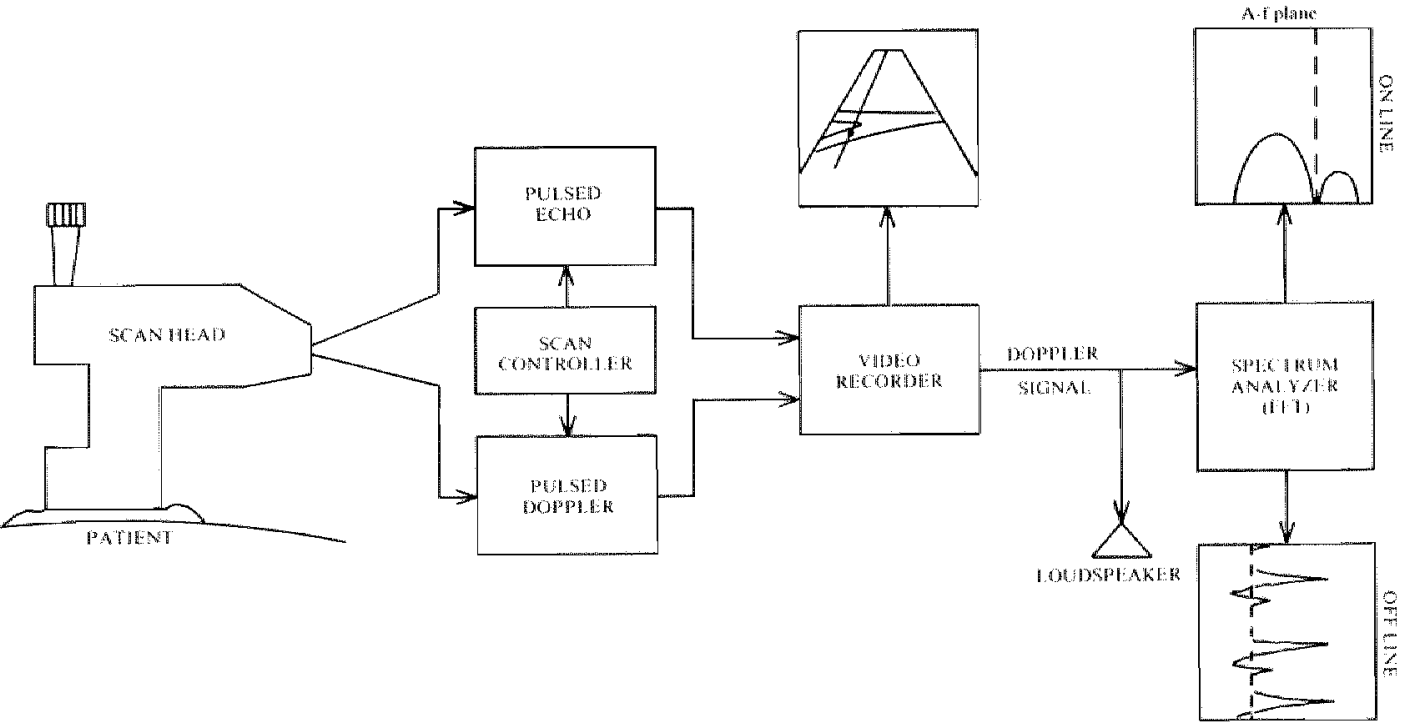

Figure $7.1 / 1$

Block diagram of the equipment set-up for recording and displaying the B-mode images and Doppler signals. 


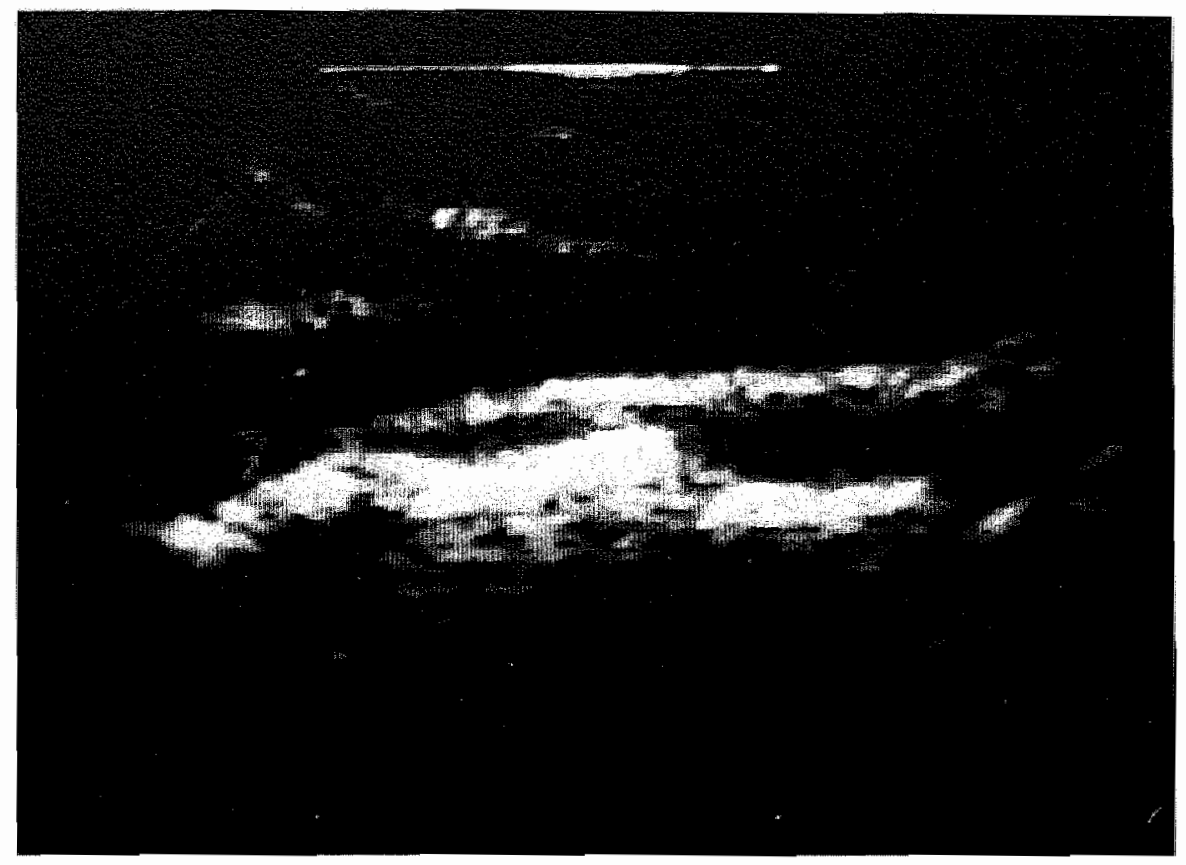

Figure $7.1 / 2$

A B-mode image of a normal carotid bifurcation. The common carotid artery is on the right side. The internal and external carotid arteries are on the left side. The sample volume is represented by the white spot and placed in the centre stream of the internal carotid artery.

beam axis of the Doppler is represented on the image by an enhanced white line; the point from which flow is sampled is represented by a cursor (figure 7.1/2). At a depth of 3 centimeters the system permits the use of a pulse repetition frequency of $19.2 \mathrm{kHz}$, which allows a Doppler frequency response of up to $9.6 \mathrm{kHz}$ before frequency aliasing occurs. At a depth of 5 centimeters these numbers are $12.8 \mathrm{kHz}$ and $6.4 \mathrm{kHz}$ respectively. Both the $\mathrm{B}$-mode image and the directional Doppler signal are recorded on tape for review and processing of the velocity signal.

Two different types of scan heads are used, the medium focus scan head $(721 \mathrm{~B})$ and the short focus scan head $(720 \mathrm{~A})$. The medium focus scan head has a $9.5 \mathrm{~mm}$ crystal diameter and a $40 \mathrm{~mm}$ focal point, whereas the short focus scan head has a $6 \mathrm{~mm}$ crystal diameter and a $20 \mathrm{~mm}$ focal point. Since the width of the ultrasound beam varies as a function of range, the sample volume is likewise range dependent. In the lateral direction, perpendicular to the ultrasound beam axis, the size of the sample volume is given by the beam pattern varying as function of the distance to the transducer surface due to 
focussing. In the axial direction, along the ultrasound beam, the size of the sample volume depends on the duration of the emitted ultrasound bursts, and the frequency characteristics of the transducer and signal processing circuitry. The size of the sample volume, relative to the diameter of the vessel being studied, has a considerable effect on the quality of the Doppler signals and, consequently, the interpretation of the spectra of the velocity wave form (Knox et al., 1982, wan Merode et al., 1983). It is therefore essential to obtain specific information about the size of the sample volume as function of the distance to the transducer, especially if the results obtained with different scan heads are to be compared. To evaluate the spatial resolution of our pulsed Doppler systems we employed the method described by Hoeks and coworkers (1984). These authors developed a method which especially is suited to evaluate the dimensions of the sample volume of pulsed Doppler systems in both axial and lateral direction. The results of these measurements are given in table $7.1 / 1$. The sample volumes, are defined as the volume at a given range from where $90 \%$ of the backscattered energy originates.

Table $7.1 / 1$

The size of the sample wolume at increasing distance from the transducer of the short focus and medium focus scan heads, respectively.

depth

sample volume $\left(\mathrm{mm}^{3}\right)$

$(\mathrm{mm})$

short focus

medium focus

\section{0}

25

30

40

50

60

70
3.3

3.4

4.0

7.4

8.8

13.5
22.6

19.7

14.2

7.5

6.5

10.5

The beam width of our medium focus scan head is narrowest from 40 to 50 $\mathrm{mm}$, whereas our short focus scan head has a focal range from 20 to $30 \mathrm{~mm}$. The sample volumes for the short and medium focus scan heads, in their focal ranges, are calculated to be 3 and $7 \mathrm{~mm}^{3}$ respectively. Therefore, the short focus scan head is more appropriate for the investigation of the carotid bifurcation which is usually located at a distance less than $30 \mathrm{~mm}$ from the transducer. At a distance of 40 to $60 \mathrm{~mm}$ from the transducer surface the spatial resolulation of our two scan heads do not differ much from each other. 
Therefore, both scan heads are appropriate for the investigation of the innominate, subclavian and vertebral arteries, when usually positioned further than $30 \mathrm{~mm}$ from the transducer.

\subsection{DATA PROCESSING}

The quadrature outputs of the pulsed Doppler signal are processed by a single channel digital fast Fourier transform spectrum analyzer (Nicolet UA$500 \mathrm{~A}$ ). The maximum range of the spectrum analyzer is $50 \mathrm{kHz}$ constructed from 500 frequency bins spaced at $100 \mathrm{~Hz}$. With a total range of $20 \mathrm{kHz}$ it uses 325 frequency bins spaced at $40 \mathrm{~Hz}$. Ordinarily we truncate the range at $13 \mathrm{kHz}$.

To enable simultaneous analysis of the forward and reverse Doppler spectra, both spectra are mixed to one single channel by means of frequencydomain multiplexing. The frequency multiplexer used for this study has been described by Ruissen (1980) and was built by the Department of Medical Electronics of the St. Antonius Hospital, Nieuwegein, The Netherlands. In this frequency multiplexer both Doppler spectra are modulated with a reference signal of a stable frequency (Fref) in a balanced modulator-demodulator. The reference frequency itself is suppressed in the modulator. The output of each modulator-demodulator is a frequency spectrum containing the original spectrum which has been shifted to a higher frequency with Fref (upper side band), plus the mirror image of the same spectrum below the Fref (lower side band). In the case of the forward frequency spectrum the upper side band is eliminated by passing the signal through a low pass filter. The reverse frequency spectrum is passed through a high pass filter, which eliminates the lower side band. Both modulated Doppler spectra are summated with each other. The resulting spectrum contains all frequencies of both original spectra, now projected in an opposite direction, with the selected reference frequency as an artificial zero scale.

Figure $7.2 / 1$ is a diagram representation of the display system for the directional Doppler spectra. It shows how the forward and reverse audio signals are channelled from the tape, on which they were recorded, through the frequency multiplexer to the spectrum analyzer.

During duplex scanning the results of this signal processing, relative power versus frequency, is continuously displayed on a Tektronix $5130 \mathrm{~N}$ oscilloscope. Off-line a hard copy is made by means of an ultraviolet recorder (Honeywell Visicorder) on Kodak Linagraph direct print paper. In this display, Doppler-shifted frequencies are shown on the vertical axis ( $\mathrm{Y}$-axis), time on the horizontal axis ( $\mathrm{X}$-axis), and the relative power per frequency as variations in the intensity or grey scale ( $Z$-axis). Basically, the grey scale coded amplitude display indicates the relative number of erythrocytes travelling at a 

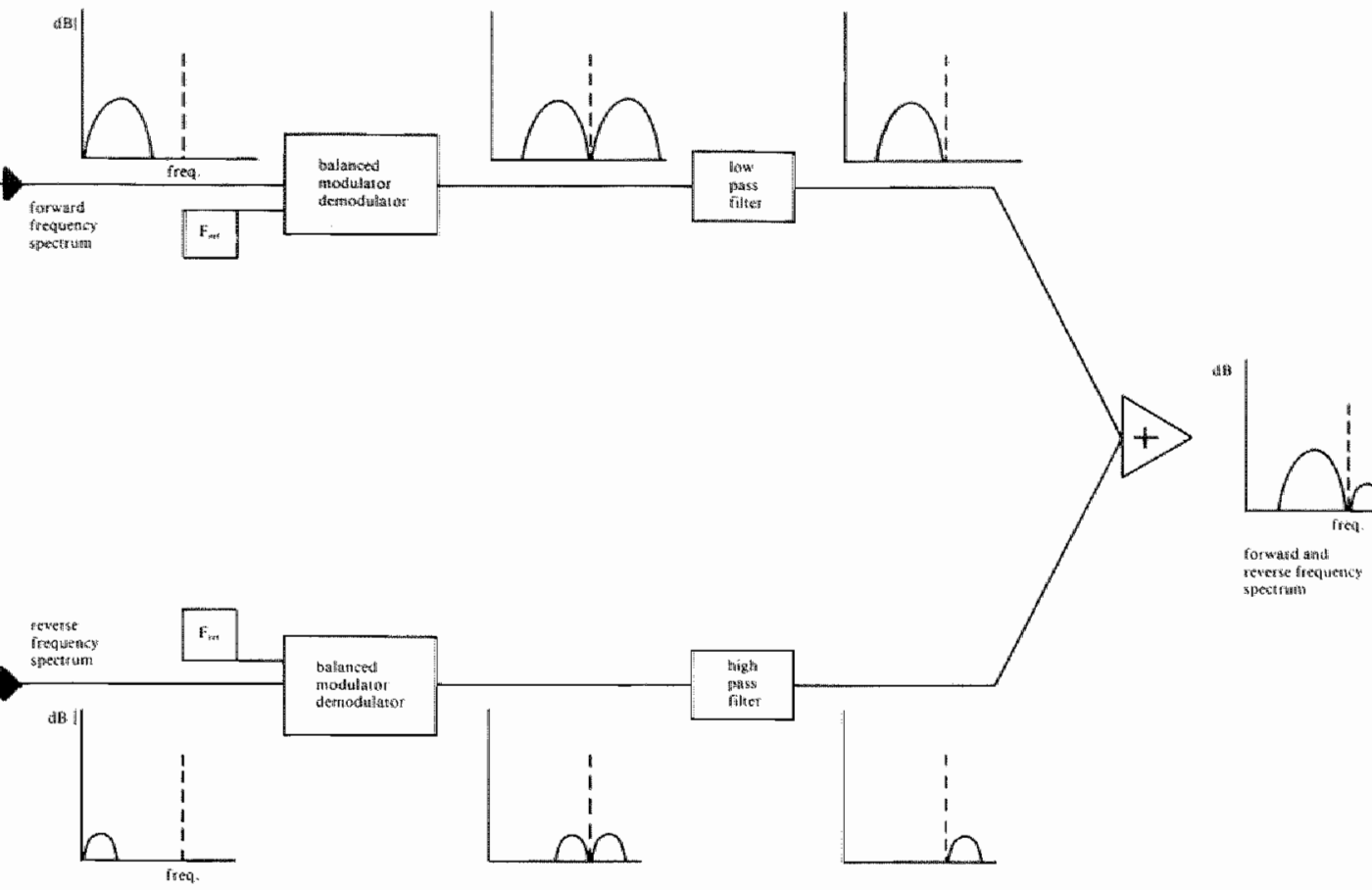

Figure $7.2 / 1$

Principle of frequency multiplexing.

specific velocity. For example, if a large proportion of red blood cells within the ultrasonic beam are moving at a certain velocity, the display will be black at the frequency level corresponding to that velocity. If a small number of red blood cells are moving at a certain velocity, the display will be light grey. The resulting display often is referred to as a sonagram or spectrogram. Routinely our recording device operates off-line and provides a full scale frequency display of $13 \mathrm{kHz}, 10 \mathrm{kHz}$ forward and $3 \mathrm{kHz}$ reverse. The forward and reverse channels can be exchanged by means of a switch.

73 


\section{ULTRASONIC DUPLEX SCANNING OF THE ARCH VESSELS AND VERTEBRAL ARTERIES}

\subsection{PATIENT EXAMINATION METHOD}

All investigations were performed during a single uninterrupted session. Before the start of the investigation the presence of bruits in the supraclavicular fossa and carotid region was searched for with a stethoscope and the result of this examination was recorded as 'absent' or 'present'. During the investigation the patient lay in a relaxed supine position with his head on a small pillow.

In the first part of the study (May 1981-December 1982) most investigations were performed by one single technician. Afterwards we trained two other technicians and during the second part of the study (December 1982October 1984) the investigations were performed at random by one of the three technicians.
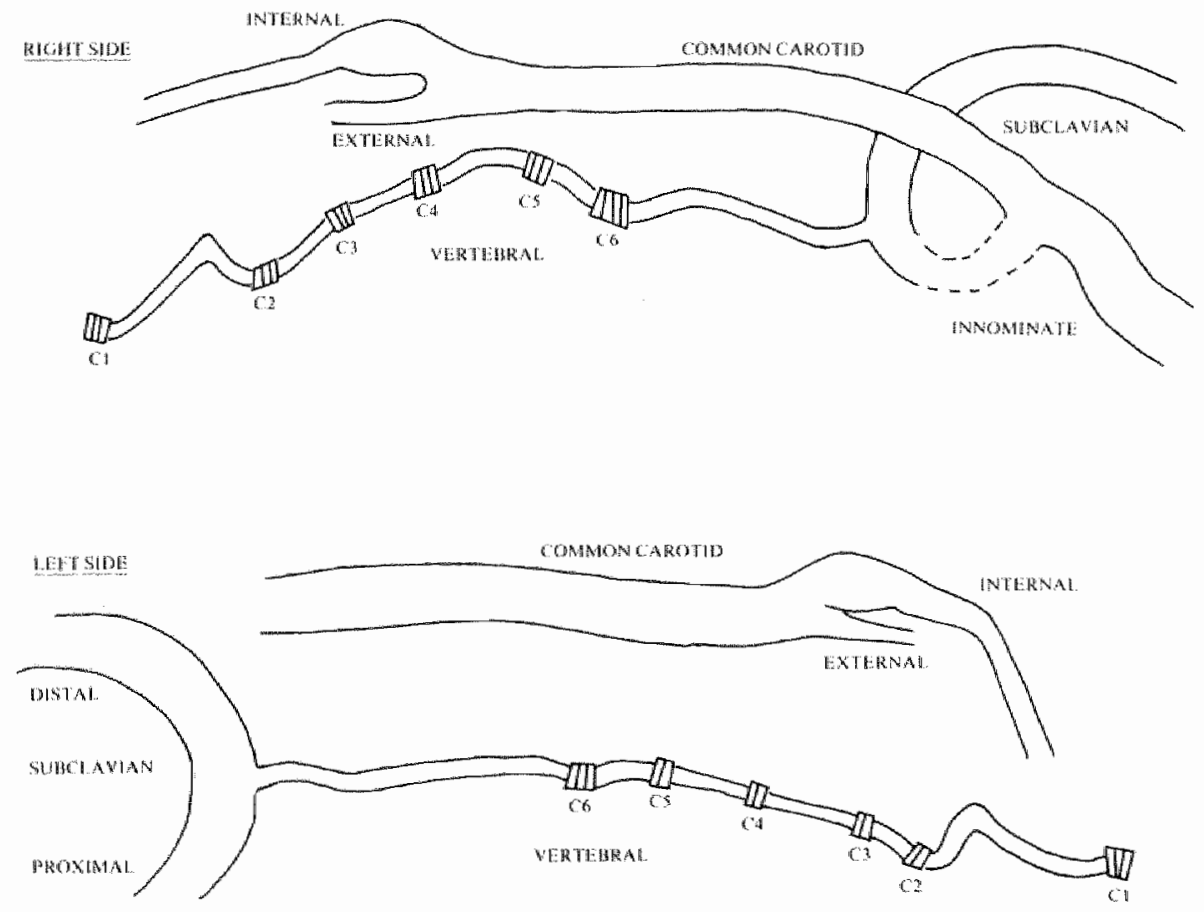

Figure 8.1/1

An example of the drawings of the extracranial cerebral arteries made by the investigator during the non-invasive study. In this case all vessels were normal. The dotted lines of the right subclavian artery stand for a well discerned proximal part of this vessel, but a Doppler measurement at the site of its origin was impracticable. 
During the investigation the technician gave an interpretation of the audio Doppler signals which was noted on a list. At the same time the technician made a drawing of the relevant cerebral vessels, composed as a photomosaic picture from the B-mode images (fig. 8.1/1 to 8.1/3). Afterwards the technician and the author himself used the videotape recordings and the sonagrams for the definitive description and conclusion.

Initially we only scanned the carotid bifurcation, but because of our interest in atherosclerotic disease of the posterior circulation we soon attempted to
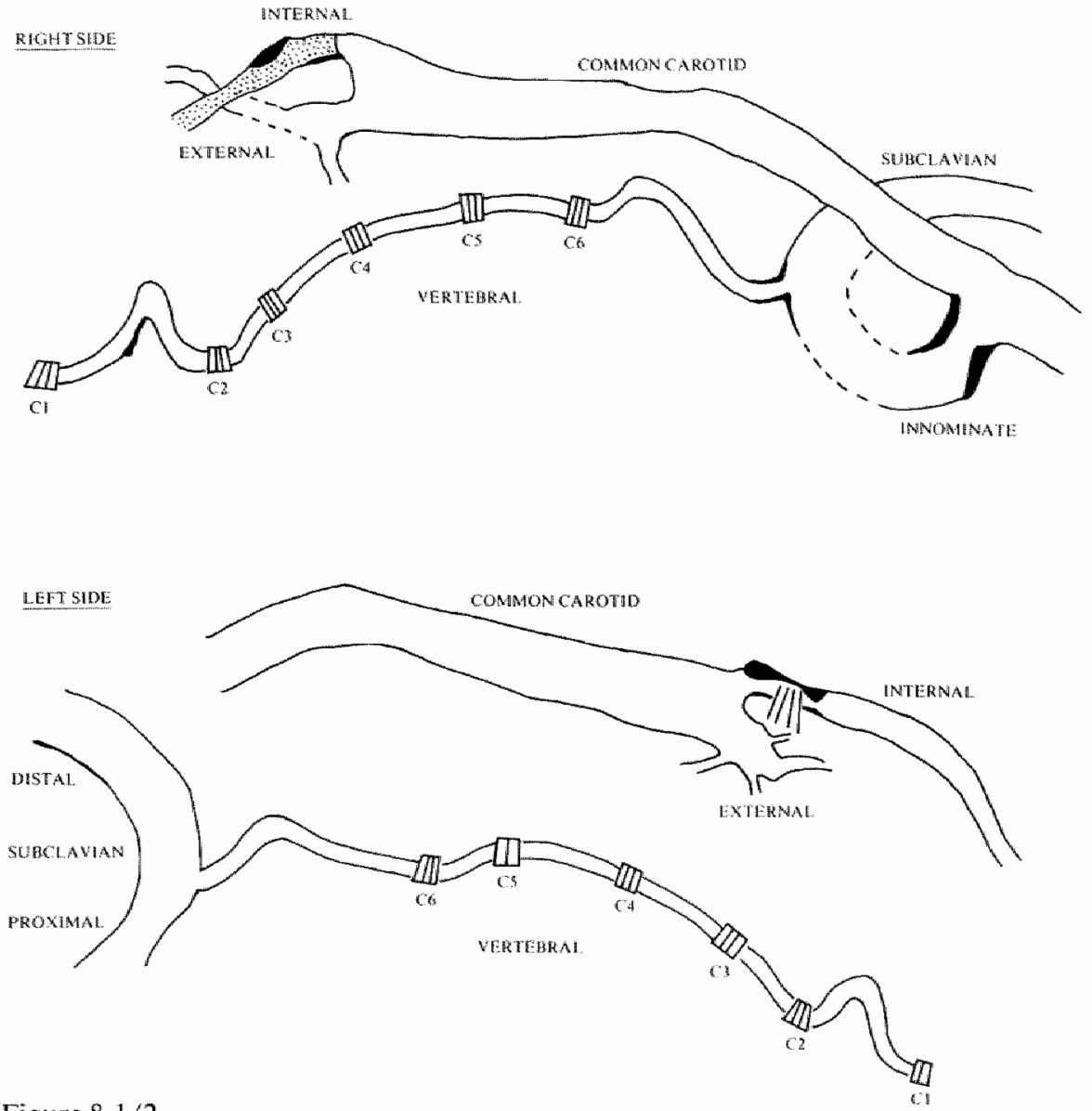

Figure 8.1/2

An example of the drawings of the extracranial cerebral arteries made by the investigator during the non-invasive study of a patient with multi-level disease. The right internal carotid artery was occluded, a pressure reducing stenosis with calcifications was located proximally in the left internal carotid artery and atherosclerotic plaques were seen at the level of the origins of the right subclavian and right vertebral arteries as well as in the most distal part of the cervical segment of the right vertebral artery. 
routinely investigate the subclavian arteries and the proximal, prevertebral segment of the vertebral artery as well. During the second part of the study we also routinely investigated the innominate artery and, in the event of a history of vertebrobasilar insufficiency, the second, cervical segment of the vertebral artery in its bony canal up to the part where it loops around the atlas.

At present the examination of the innominate, subclavian, and vertebral arteries is part of an extensive ultrasonic duplex scanning of the extracranial cerebral vessels. Routinely ten or eleven Doppler measurements are performed on each side, that is in the

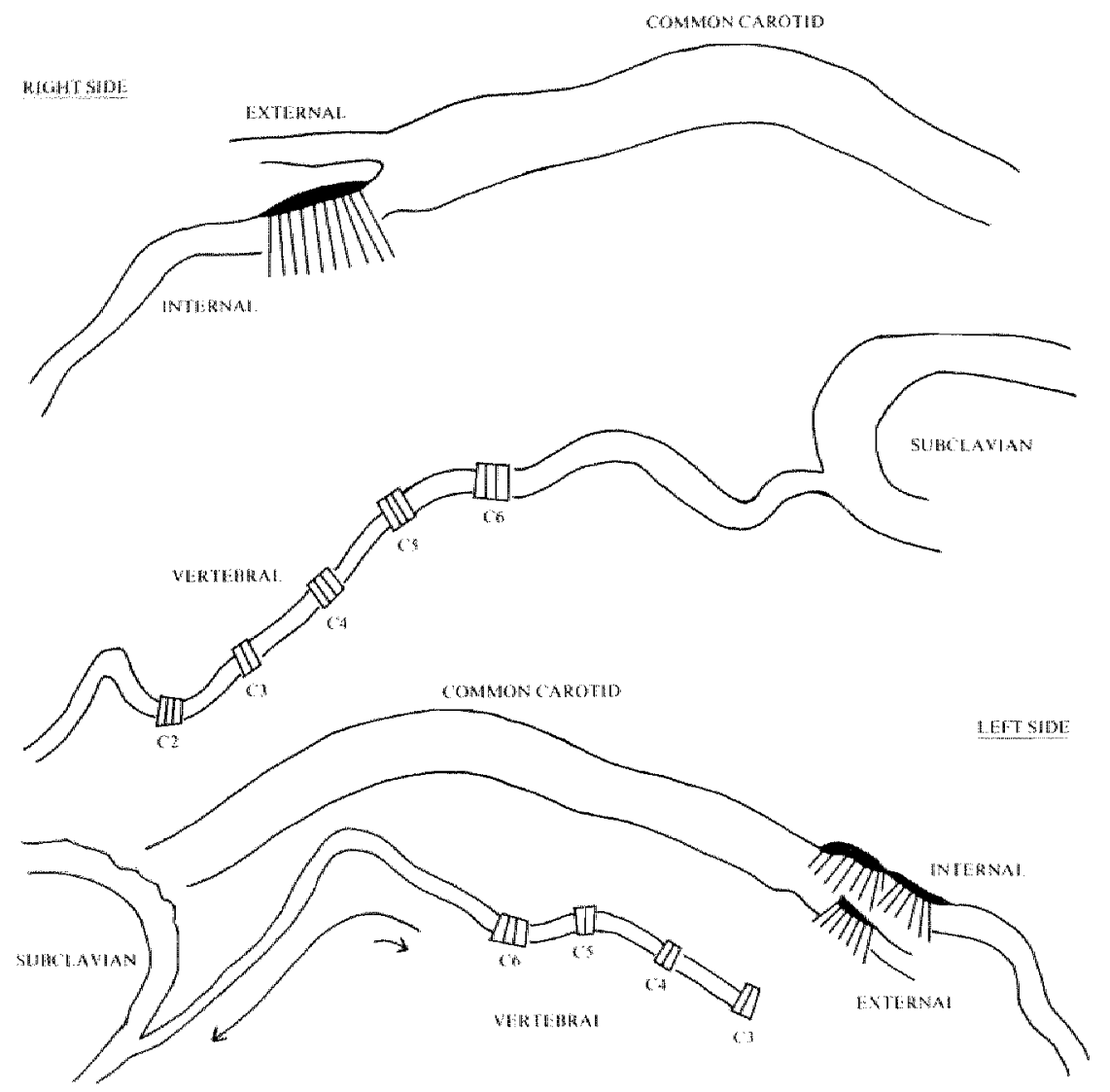

Figure $8,1 / 3$

Another example of the extracranial part of the cerebrovascular tree of a patient with multimlevel disease. In this case it was not possible to image the innominate bifurcation. On both sides atherosclerotic lesions with extensive calcifications were seen in the carotid bifureations. On the right side there was a normal subclavian artery and a relative robust vertebral artery. On the other hand, the left subclavian artery showed severe atherosclerotic changes and there was a transient subclavian steal syndrome on the left side. 
- distal part of the innominate artery

- proximal and more distal parts of the subclavian artery

- proximal and distal parts of the common carotid artery

- proximal part of the external carotid artery

- proximal, mid, and distal parts of the internal carotid artery

- proximal and distal parts (just proximal to 66 ) of the vertebral artery.

If the examination includes the investigation of the cervical segment of the vertebral artery, Doppler measurements are performed on both sides between all transwerse processes of the cervical vertebrae up to the atlas.

\subsubsection{THE CAROTID SYSTEM}

We routinely begin the examination on the right side with scanning of the right common carotid artery. By moving the probe distally and scanning in various planes the carotid bifurcation can be recognized with usually the external carotid artery in an anteromedial and the internal carotid artery in a posterolateral position. The differentiation between the external and internal carotid artery is made by the anatomical location of these vessels, the bulb of the internal carotid artery, and the characteristic Doppler signals which can be recorded from these arteries. Flow in the internal carotid artery is normally quasi-steady and above zero during the entire cardiac cycle due to the low resistance of the cerebrovascular bed. Flow in the external carotid artery, supplying a vascular bed with a higher peripheral resistance, is relatively high in systole and low in diastole. It is especially this difference in the characteristics of the Doppler spectra which makes it possible to accurately differentiate between the internal and external carotid arteries.

There is no essential difference between ultrasonic duplex scanning of the carotid arteries on the right and the left side, with the exception that the investigation of the ostium and most proximal part of the left common carotid artery is thardly ever possible.

\subsubsection{THE INNOMINATE ARTERY}

By scanning the right common carotid artery longitudinally this vessell is followed in a proximal direction as far as possible. Moving the probe towards the supraclavicular fossa the common carotid artery turns medially and posteriorly out of the plane of scanning and is suddenly seen in a transverse direction. At this moment the right subclavian artery is often situated transversely just posterolateral to the common carotid artery. By moving the probe slightly more proximally both vessels unite and a transverse image of the distal segment of the innominate artery is seen. Often it is necessary to move 


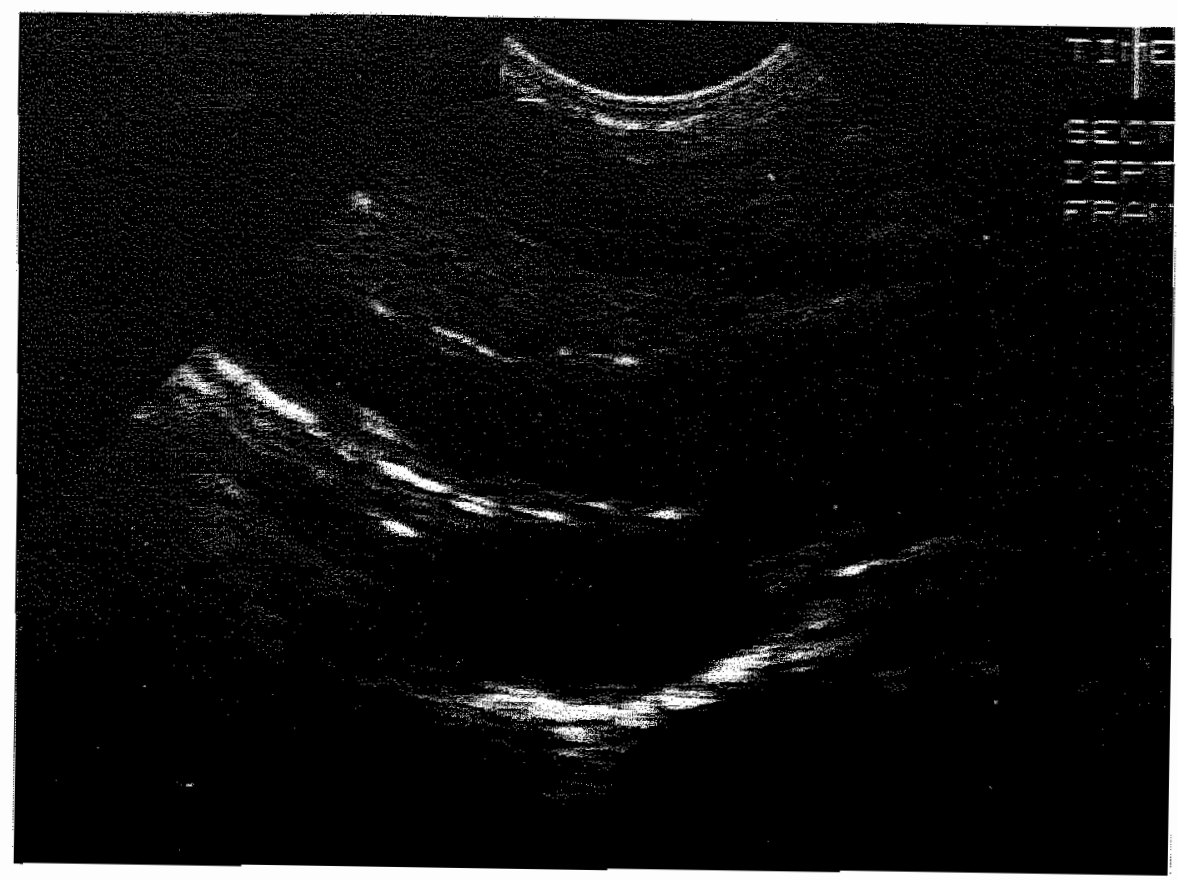

Fingure 8.1.2/1

The innominate bifurcation, the most proximal part of the right common carotid artery is located on the left side, passes to the right and enlarges into the distal part of the innominate artery. The most proximal part of the right subclavian artery is also imaged and shows an atherosclerotic lesion this origin.

the probe to the medial side of the sternocleidomastoid muscle towards the suprasternal notch.

Usually it is also possible to obtain longitudinal images of the segments of the innominate bifurcation by following the longitudinal axis of the right common carotid artery with the plane of scanning (fig. 8.1.2/1). By changing the position of the probe step by step the proximal segment of the right common carotid artery suddenly enlarges to pass into the distal segment of the innominate artery. With the probe in this position it is even possible to visualize the most proximal part of the right subclavian artery as a small band on the posterolateral wall of the innominate artery (fig. $8,1.2 / 2$ ). The visualization of the innominate bifurcation depends on local anatomical properties such as the exact location of the arch of the aorta and the length of the innominate artery. However, in most patients it is possible to visualize the distal segment of this artery and to perform a Doppler measurement on it. 


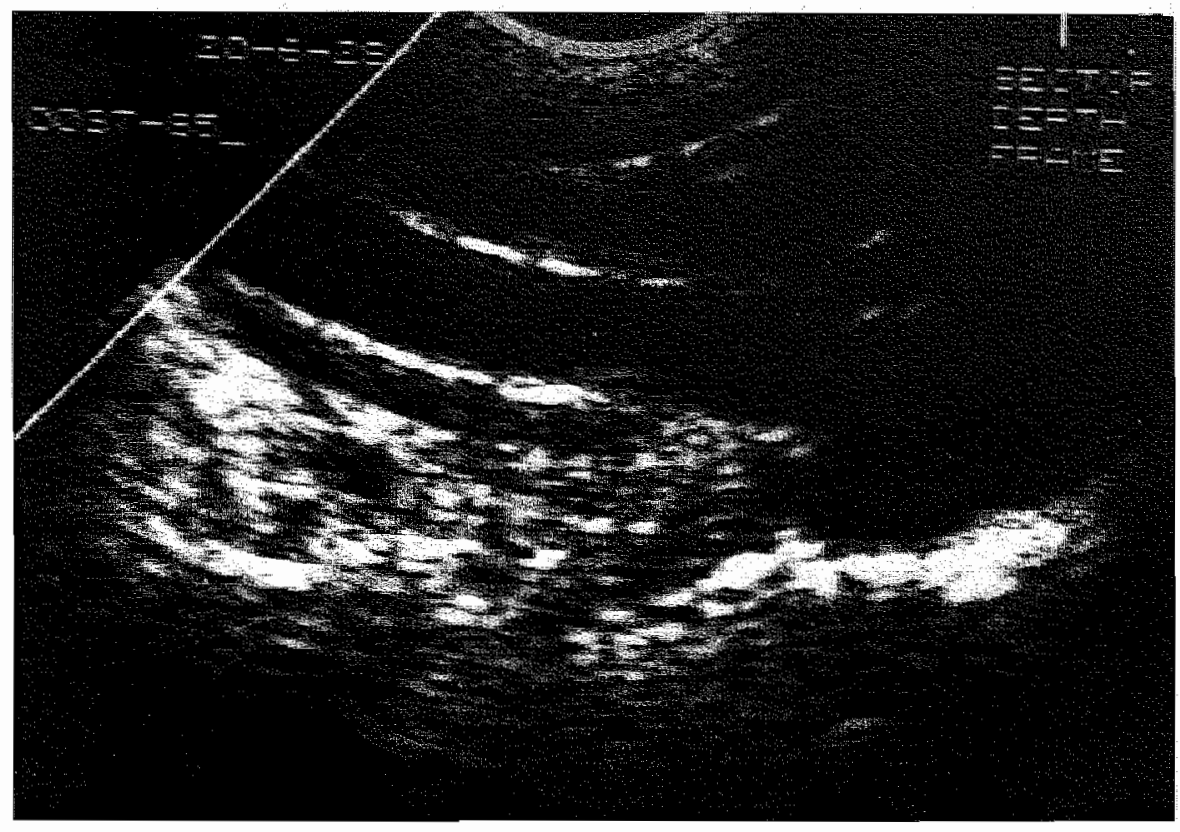

Figure $8.1 .2 / 2$

Another example of the innominate bifurcation. In this case only a very small part of the right subclavian artery is imaged. The right vertebral artery originates proximally from the subclavian artery and runs distally parallel to the right common carotid artery.

\subsubsection{THE SUBCLAVIAN ARTERIES}

With the probe in the aforementioned position it is possible in many cases to longitudinally visualize the origin and proximal segment of the right subclavian artery. In patients with atherosclerosis of this part of the extracranial cerebral vascular tree, wall irregularities and atherosclerotic plaques are often well discerned. However, this part of the right subclavian artery makes a posterolateral curve and, in spite of many efforts, the axis of the wessel of ten remains perpendicular to the Doppler beam. A Doppler measurement at the side of the ostium or in the very first proximal part of the right subclavian artery is then impracticable. The investigation of the more distal part of the first segment of the right subclavian artery is usually not a problem. It is relatively simple to visualize this part of the artery along its longitudinal axis and a Doppler measurement can easily be performed. 
As a result from the fact that it originates from the aortic arch the ostium and the most proximal part the left subclavian artery is more posteriorly and more caudally located than on the right side. According to our experience ultrasonic duplex scanining of the first centimeters of the left subclavian artery is impracticable in most patients. On the other hand, the investigation of the segment adjacent to the ostium of the left vertebral artery is usually not a problem.

It must be emphasized here that atherosclerotic lesions un the subclavian artery are often localized in its most proximal part. Disturbances of the shape - of sonagrams of Dopplar spectra recorded distally to a stenosis disappear further downstram. In general, the less severe the stenosis the more rapid the transition from disturbed to normal. It is therefore important to perform Doppler measurements in this artery as proximally as possible and at discrete intervals more downstream.

\subsubsection{THE VERTEBRAL ARTERIES}

In the first part of our study we started the investigation of the vertebral artery by visualization of the subclavian artery. This vessel was then followed in a distal direction until the vertebral artery was found. By using this approach it is important to realize that, in contrast to the thyrocervical trunk, the vertebral artery often has a typical flat curve just distal to its origin. Although it was possible to find the ostium and follow the course of the vertebral artery in many cases, sometimes the images and Doppler recordings where inadequate and occasionally signals were mistakenly recorded from other arteries.

Therefore, we have changed the approach of the vertebral artery by visualization of the artery itself before it enters its bony canal just caudal to the sixth cervical vertebra. The acoustic shadow of the transwerse process of the sixth cervical vertebra is easily found by placing the probe on the lateral side of the neck a few centimeters above its base. With the plane of scanning along the longitudinal axis of the neck it is possible to obtain an image of the transverse process of the sixth cervical vertebra as a black shadow in the cranial part of the B-mode image (fig. $8.1,4 / 1$ ). When the probe is turned slightly laterally or medially, the vertebral artery can be visualized just caudal to the point where it enters the bony canal. By changing the position step by step, one can follow the vertebral artery along its longitudinal axis in a proximal direction down to its origin from the subclavian artery. Usually the subclavian artery is then visualized in an oblique or transverse plane. Figure 8.1.4/2 shows the typical course of the prevertebral segment of the vertebral artery just distal to its origin. In many cases it is possible to follow the course of the artery and precisely locate the ostium. Usually the axis of the vessel 


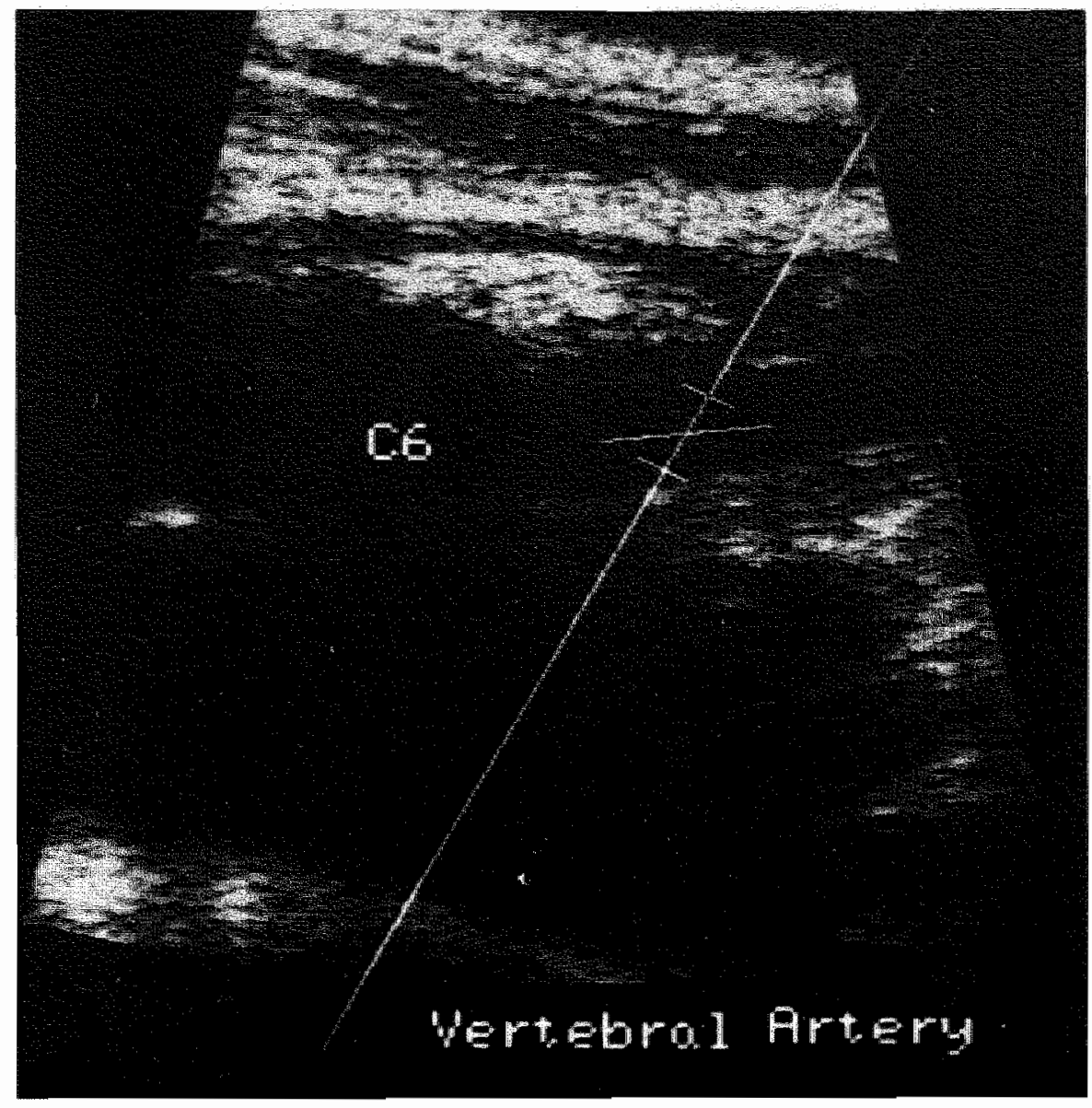

Figure 8.1.4/1

The vertebral artery passes from right to left and runs through the transverse process of the sixth cervical vertebra.

relative to the Doppler beam is less than 90 degrees and a reliable Doppler measurement is possible. Sometimes in spite of countless manipulations with the probe, the axis of the vessel remains perpendicular to the Doppler beam and a Doppler measurement is impracticable. As a result of local anatomical variations the investigation of the ostium of the left vertebral artery is generally more difficult and unsuccessful than on the right side. The distal part of the prevertebral segment of the vertebral artery is well discerned and the direction of flow can be detected, but the ostium itself lies too deeply. Thus, it may be out of the range of the pulsed Doppler device and it is therefore not possible to measure the velocity of flow near the ostium in these cases. 


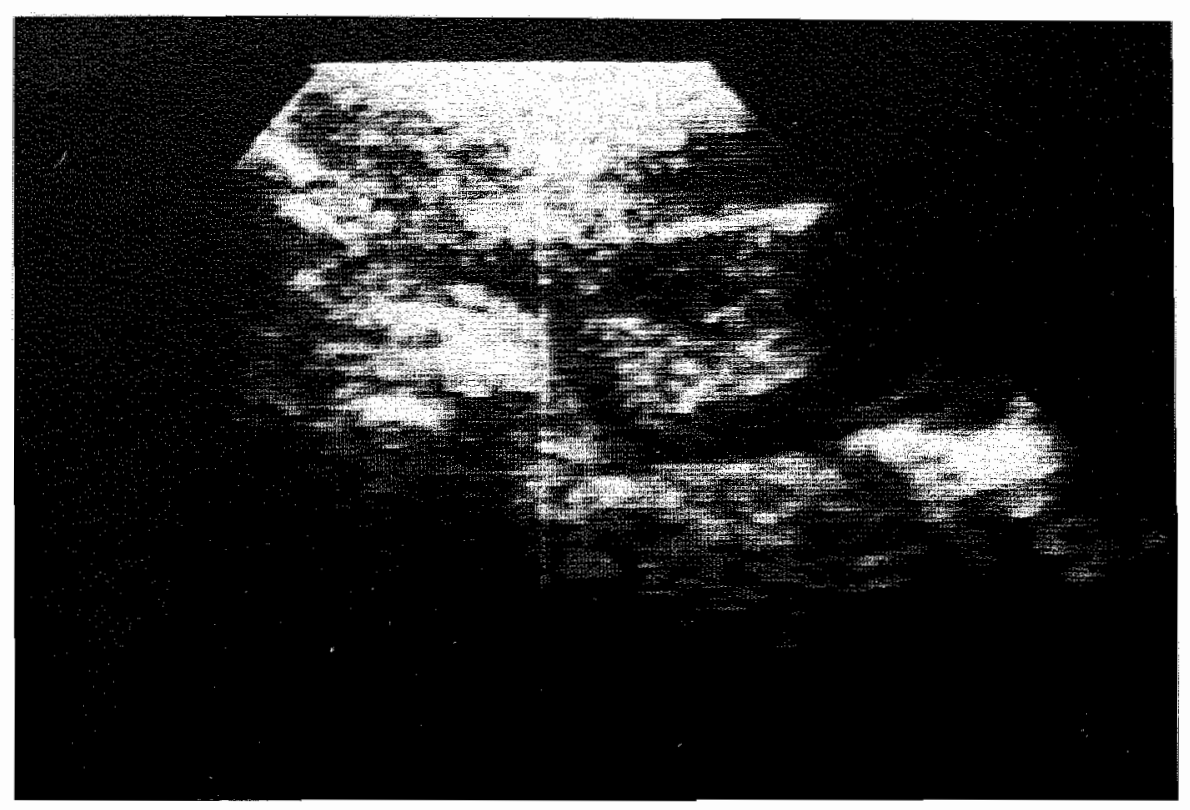

Figure 8.1.4/2

A B-mode image of the subclavian and the most proximal part of the vertebral artery. The subclavian artery is on the right side and partially seen in a transverse plane. The wertebral artery is visualized along its longitudinal axis. The sample volume is represented by the white spot and is placed in the second curve of the vertebral artery.

In experienced hands, it is even possible in many patients to investigate the cervical segment of the vertebral artery in its bony canal until it loops around the atlas. By moving the probe cranially one can visualize the transverse processes of the cervical vertebrae one by one and in between them the short segments of the vertebral artery (fig. 8.1.4/3). Normally investigation of this segment of the vertebral artery is not a problem as far as the transverse process the third cervical vertebra. However, the part between the third and second cervical vertebrae is located a little more deeply and therefore visualization of this segment is more difficult. In many cases the segment between the axis and atlas can be visualized very well and sometimes disturbances of the Doppler signal in this segment can be heard by turning the head to the contralateral side. 


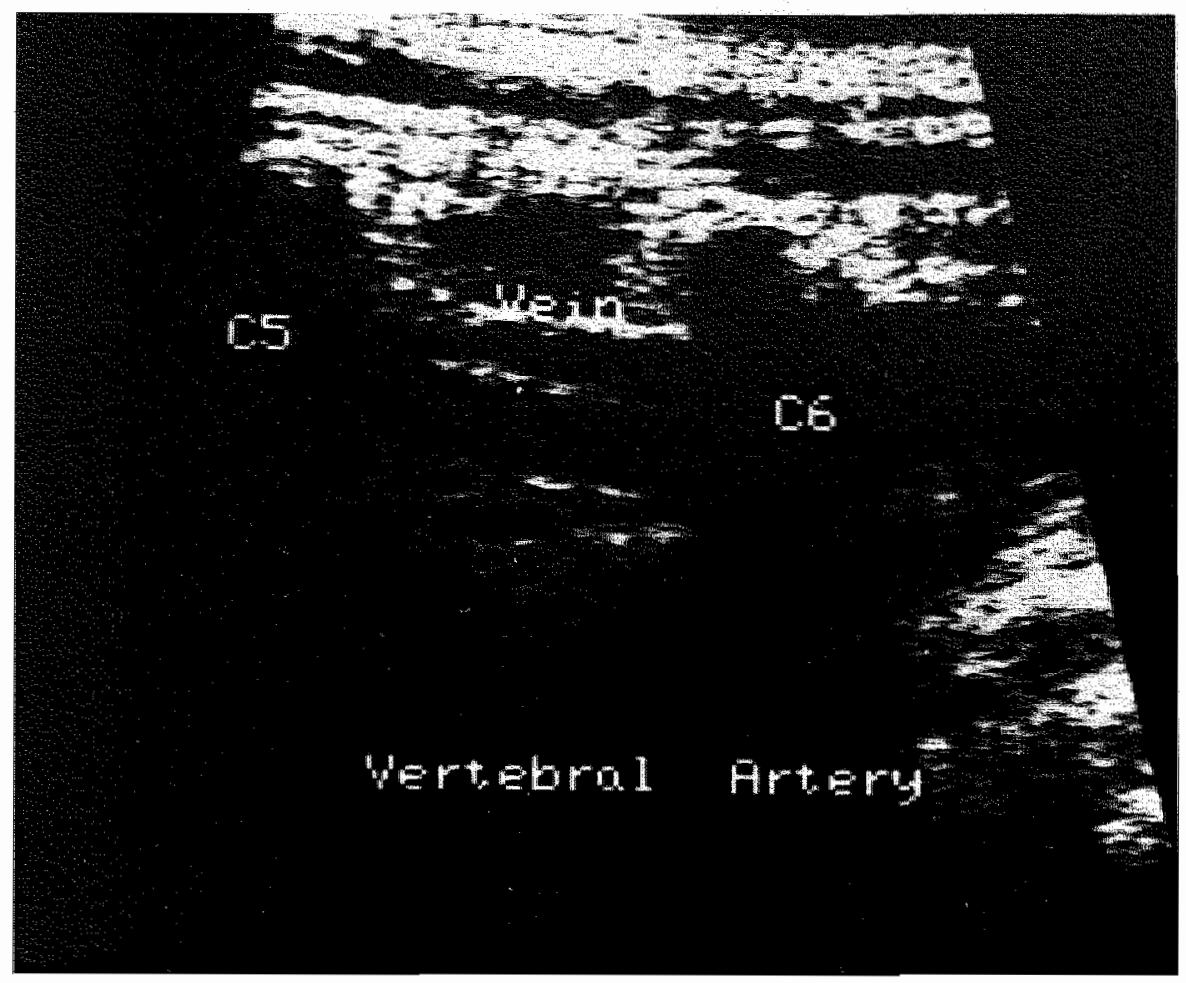

Figure 8.1.4/3

The vertebral artery and vein are imaged between the transverse processes of the sixth and fifth cervical vertebrae.

\subsection{CRITERIA AND CATEGORIES USED FOR DIAGNOSIS}

The possibilities and limitations of ultrasonic duplex scanning of the internal and external carotid arteries are well described (Fell et al., 1981, Breslau, 1982, Knox et al., 1982-b, Ackerstaff et al., 1982).

The ability to define the walls of vessels within a two-dimensional ultrasonic image and to employ a pulsed Doppler system to evaluate the blood flow velocities at any site within the visualized artery makes this method accurate. The B-mode image of the vessels of interest is an anatomical guide by which one is informed about the course and location of the arteries. Moreover, in many cases the ultrasonic image provides useful information about atherosclerotic plaques within and along the vascular tree. However, the assessment of arterial disease by this method depends upon the interpretation of spectral changes in the sonagrams recorded from specific sites along the arteries. The 


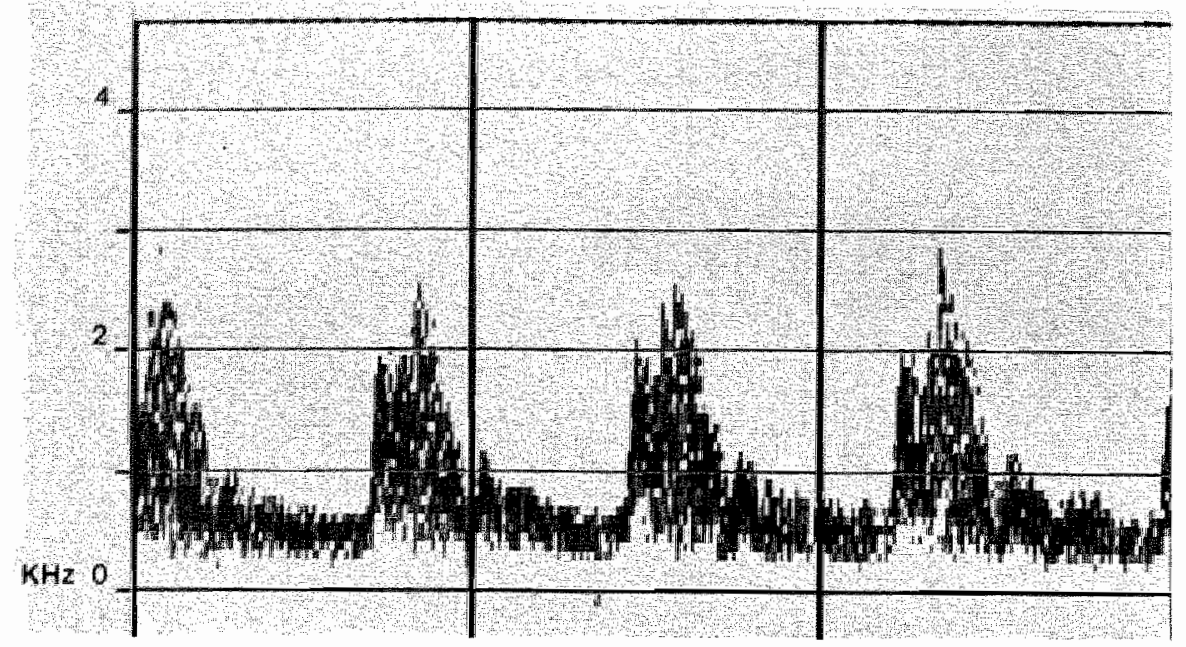

Figure $8.2 / 1$

The Fourier transform of the Doppler signals of a normal vertebral artery. As the sample volume is large relative to the vessel diameter, there is usually spectral broadening during systole.

operator measures the angle of the Doppler beam relative to the axis of the vessel of interest. An angle of approximately 60 degrees provides a favourable return signal for further processing.

To avoid detection of velocities and eddies close to the vessel wall, in large vessels such as the subclavian or innominate arteries, the sample volume is placed near the centre stream. Under normal conditions with a laminar flow during systole the red blood cells are moved forward with more of less the same velocity in the centre of the stream. Therefore, the sonagram shows a narrow band of frequencies with a clean window below the systolic peak (fig. $8.2 / 3$ and $8.2 / 4$ ). With regard to the much smaller vertebral arteries one always obtains reflections from the slowly moving red blood cells near the vessel wall. This results in an increase of the spectral width and the window below the peak is fillid in (fig. 8.2/1). This phenomenon is called spectral broadening and in ultrasonic duplex scanning of the carotid arteries it is supposed to be a sign of minor wall abnormalities. However, in our experience it is a normal finding in vertebral arteries.

The spectral changes evaluated include peak systolic frequency, the amount of spectral broadening during systole, the overall shape of the sonagrams, and flow direction (forward, zero, or reverse flow). 


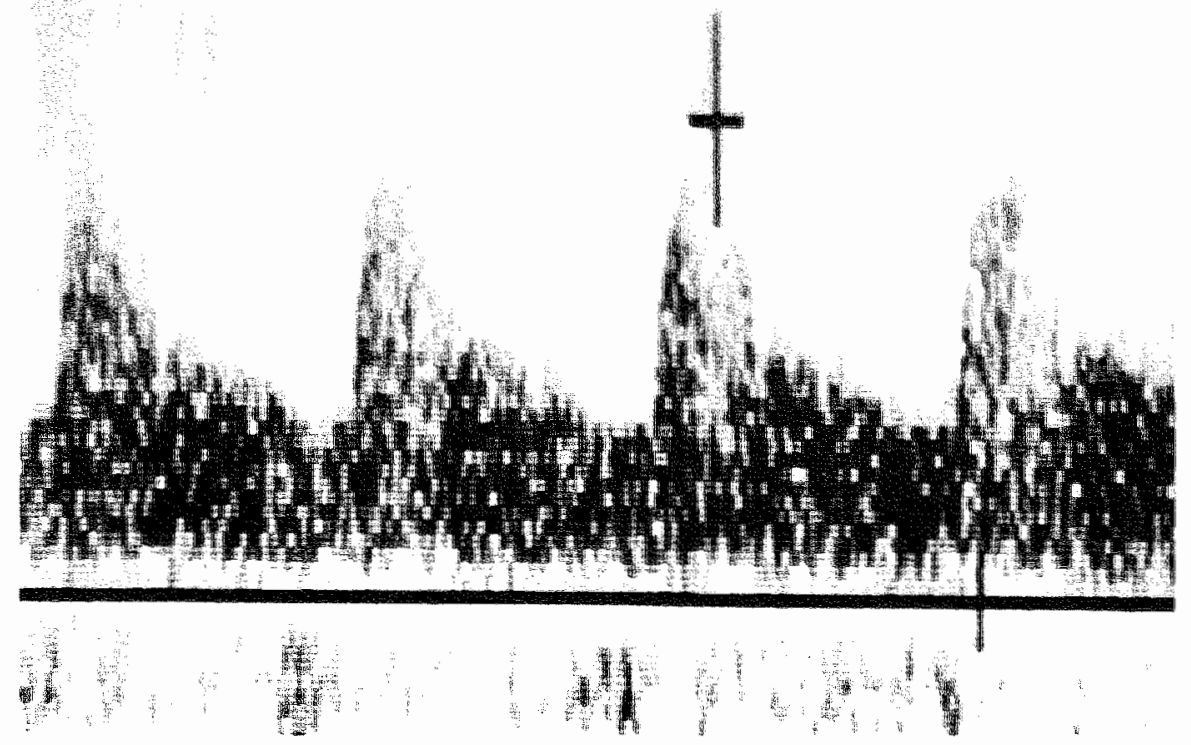

Figure $8.2 / 2$

The Fourier transform of the Doppler signals of a vertebral artery with a significant stenosis at the site of the ostium. Note the increased peak frequencies (cross $5.3 \mathrm{kHz}$ ) and spectral broadening.

Due to the low peripheral resistance to flow in the vertebral artery which supplies the brain with blood, there is a forward flow during the whole cardiac cycle. Reversed flow in the vertebral artery is a sign of severe pathology. The cause of flow reversal is a haemodynamically significant stenosis of the innominate artery or of the subclavian artery proximal to the ostium of the vertebral artery. In vertebral arteries flow reversal is sometimes cardiac cycle dependent (von Reutern et al., 1978).

According to the characteristic spectral changes of the sonagrams recorded in the vertebral artery, three categories can be defined:

(a) NORMAL. Physiological antegrade direction of flow, peak frequencies less than $4 \mathrm{kHz}$ and moderate spectral broadening during systole without striking turbulence (fig. $8.2 / 1$ ).

(b) ABNORMAL. Physiological antegrade direction of flow, peak frequencies more than $4 \mathrm{kHz}$, an increased spectral broadening and striking turbulence during systole (fig. $8.2 / 2$.). 


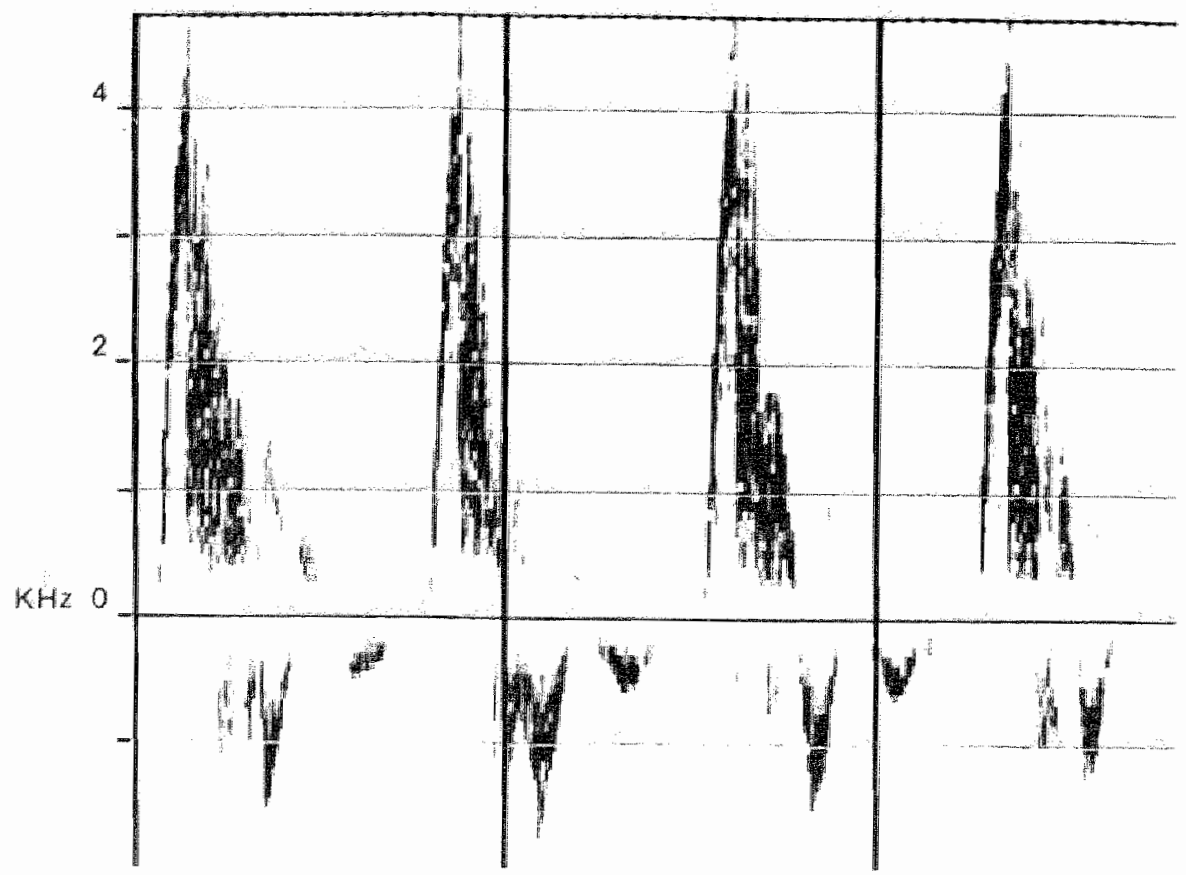

Figure 8.2/3

The Fourier transform of the Doppler signals of the subclavian artery.

(c) TOTAL OCCLUSION. Usually no image of the vertebral artery is acquired and a Doppler measurement is impracticable.

The parameter of $4 \mathrm{kHz}$ only applies with the use of a transducer with a nominal frequency of $5-\mathrm{MHz}$ and an angle of insonation of 60 degrees. It is important to emphasize here that in this study a normal Doppler signal means a normal vessel or a vessel with a stenosis with a diameter reduction of less than $50 \%$. An abnormal Doppler signal means a vessel with a stenosis with a diameter reduction of 50-99\%.

For the innominate and subclavian arteries the criteria are somewhat different. The flow patterns in these arteries show a steep systolic wave and after the onset of diastole a brief period of flow reversal. During diastole there is no significant flow present in the subclavian artery (fig. $8.2 / 3$ ). In the innominate artery, however, forward flow is usually observed during diastole (fig. $8.2 / 4$ ). Under normal conditions the peak frequencies obtained in the arch vessels are often higher than $4 \mathrm{kHz}$. If the sample volume is placed near the centre stream the recorded Doppler signals are clean. With increasing stenosis 


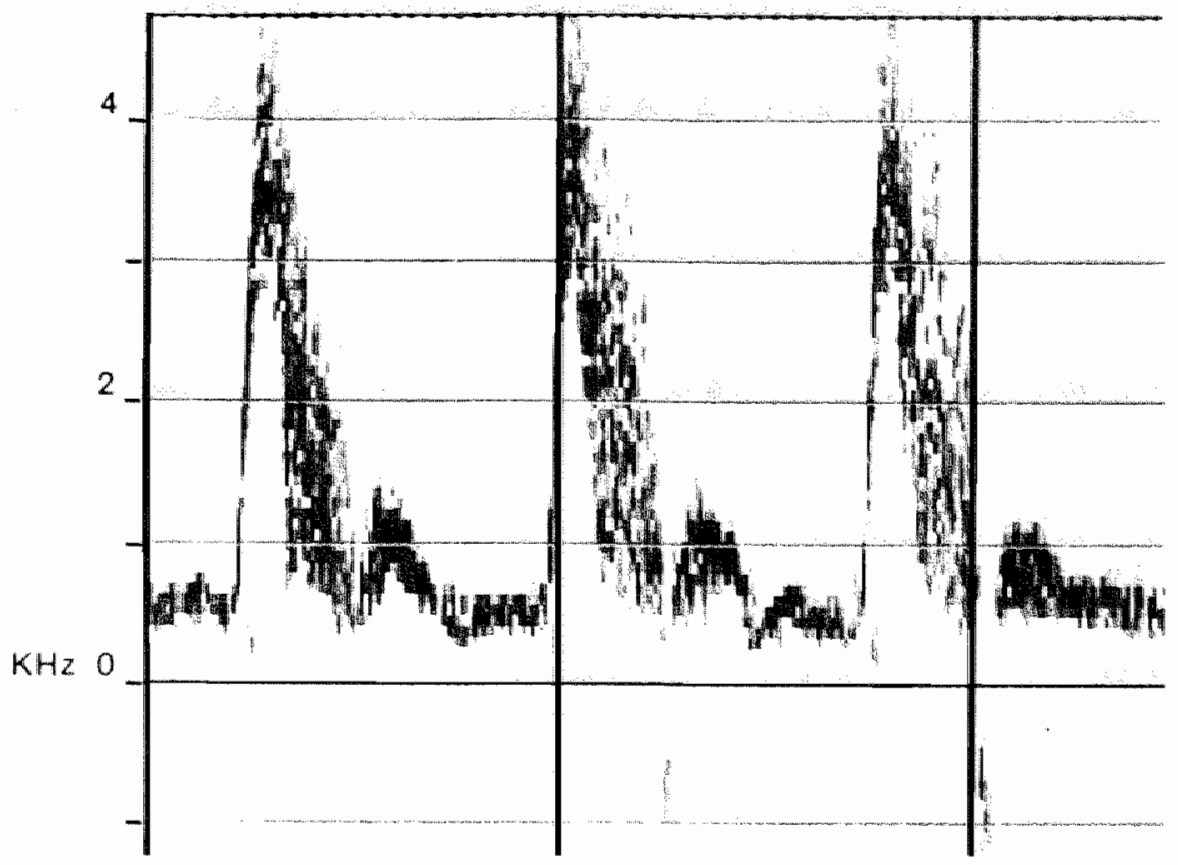

Figure $8.2 / 4$

The Fourier transform of the Doppler signals of the innominate artery. Note the forward flow during diastole.

there is more and more turbulence. In this case the window below the systolic peak of the sonagram is filled in and sometimes reversed flow during systole is seen. If an obstructive lesion is located proximally in the subclavian or innominate artery, it is not always possible to place the sample volume near the stenotic area. The outline of the sonagram is irregular a few centimeters downstream to a significant stenosis and it often reveals loss of high frequencies (fig. 8.2/5). The sonagram returns to normal further downstream and in general the less severe the stenosis the more rapid is this normalization.

In the event of a total occlusion of the subclavian artery with a subclavian steal syndrome, usually one finds a low systolic tlow velocity in the poststenotic segment of the subclavian artery without flow in any direction during diastole. A remarkable asymmetry of the sonagrams is very important for the judgement of abormalities of this kind in the subclavian arteries. 


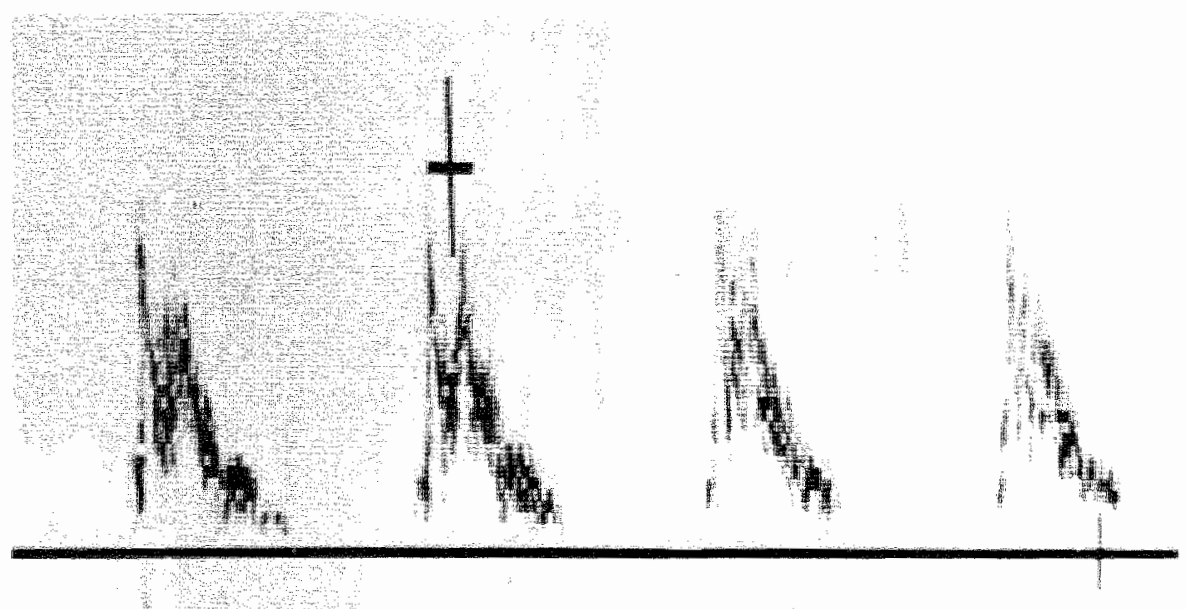

Figure $8.2 / 5$

The Fourier transform of the Doppler signals of a subclavian artery, a few centimeters downstream to a significant stenosis. Note the irregular outline and loss of high frequencies (cross $3.1 \mathrm{kHz}$ ). 


\section{THE ACCURACY OF ULTRASONIC DUPLEX SCANNING OF THE INNOMINATE, SUBCLAVIAN, AND VERTEBRAL ARTERIES, AS COMPARED WITH CONTRAST ARTERIOGRAPHY}

\subsection{INTRODUCTION}

The accuracy of ultrasonic duplex scanning of the innominate, subclavian, and vertebral arteries as compared with contrast arteriography will be presented and discussed in this chapter. More detailed information about the angiographic examinations used in this study, their numerical description as well as the statistical analysis employed will be given in section 9.2.

Two studies form the basis of the evaluation of the data. During the first study*, from May 1981 to December 1982, we only investigated the proximal segment of the subclavian and vertebral arteries. In this part of the study we compare the arteriographic data of the ostium of both the subclavian and vertebral arteries with the data of ultrasonic duplex scanning. The results of this comparison will be presented in section 9.3 .

During the second study, from December 1982 to October 1984, we also investigated the innominate artery and the cervical segment of the vertebral artery. In the sections 9.4.1 to 9.4.3 a survey is given of the data of ultrasonic duplex scanning of the innominate and subclavian arteries and of the ostium and remaining part of the prevertebral segment of the vertebral artery compared with the data of contrast arteriography. In addition the results of the investigation of the cervical segment of the vertebral artery will be presented in section 9.4.4. In this part of the study we paid particular attention to the relationship between cervical spondylosis and atherosclerotic obstructive lesions of the cervical segment of the vertebral artery. In section 9.4.5 the influence of rotational obstruction of the vertebral artery at the atlanto-axial articulation will be briefly discussed. The significance of ultrasonic duplex scanning in the event of severe atherosclerosis of the intradural segment of the vertebral artery will be delineated in section 9.4.6. Finally, the subclavian and innominate steal syndromes found during the second study will be mentioned in section 9.4.7.

\subsection{ARTERIOGRAPHIC EXAMINATIONS, THEIR NUMERICAL DESCRIPTION, AND STATISTICAL ANALYSIS USED}

The arteriographic examination of the patients was normally performed as a four-vessel arteriography using the Seldinger technique. Arch aortography, usually in two planes, was performed prior to selective catheterization of the arch vessels. Apart from biplane carotid arteriography, in many patients 
there was a semiselective study of the vertebrobasilar system, sometimes with cineroentgenography of the proximal segment of the vertebral artery. In most patients both extra- and intracranial vessels were visualized. For an estimate one has to realize that in the event of a normal ostium of the left vertebral artery a selective study of the right ostium was not routinely performed. In our hospital, we used intra-arterial digital subtraction angiography. Selective studies of the ostium of a vertebral artery in different oblique views could be performed relatively easily. If the arteriographic examination was performed in another hospital and the pictures were of satisfactory quality the results were used in our study. We only decided upon the status of the origin of an artery if the arteriographic study showed at least one picture without superposition of the most proximal part of this artery by other structures. This is an especially important point to be considered for the vertebral artery. It was the most important reason for the relative high number of arteriograms which were useless for an estimate of the percentage of stenosis at the site of the origin of the vertebral arteries.

The method of assessment of the percentage of stenosis which is used will invariably influence the test results. We used the grading protocol as indicated by Blaisdell and co-workers (1969). The degree of arterial stenosis was measured by selecting a normal appearing segment of an artery just proximal or distal to the filling defect seen on the arteriogram. As shown in figure $9.2 / 1$ the narrowest diameter within the stenosis was compared to the normal diameter and this was converted into an estimate of the percentage of stenosis. Both unsubtracted and subtracted arteriograms were used. If the stenosis diameter differed considerably in different views the most severe stenosis was taken for the final grading. For the validation studies four categories of diameter reduction were used (fig. 9.2/1).

The radiologists estimating the degree of diameter reduction were unaware of the results of ultrasonic duplex scanning. The technicians performing the non-invasive studies and the interpreter were unaware of the results of contrast arteriography.

For the present study, four categories of diameter reduction were used on arteriography (fig. 9.2/1). The data of ultrasonic duplex scanning was classified as normal or abnormal (section 8.2). The results of the classification procedures were expressed as percentages correct classifications. In the case of a simplification of the classification procedure to a two-class problem it is possible to calculate not only the proportion of correct classifications (overall accuracy), but also the diagnostic sensitivity and specificity and the positive and negative predictive values of the ultrasonic study as compared with contrast arteriography. These parameters were calculated according to the use of the decision matrix as shown in figure $9.2 / 2$. 


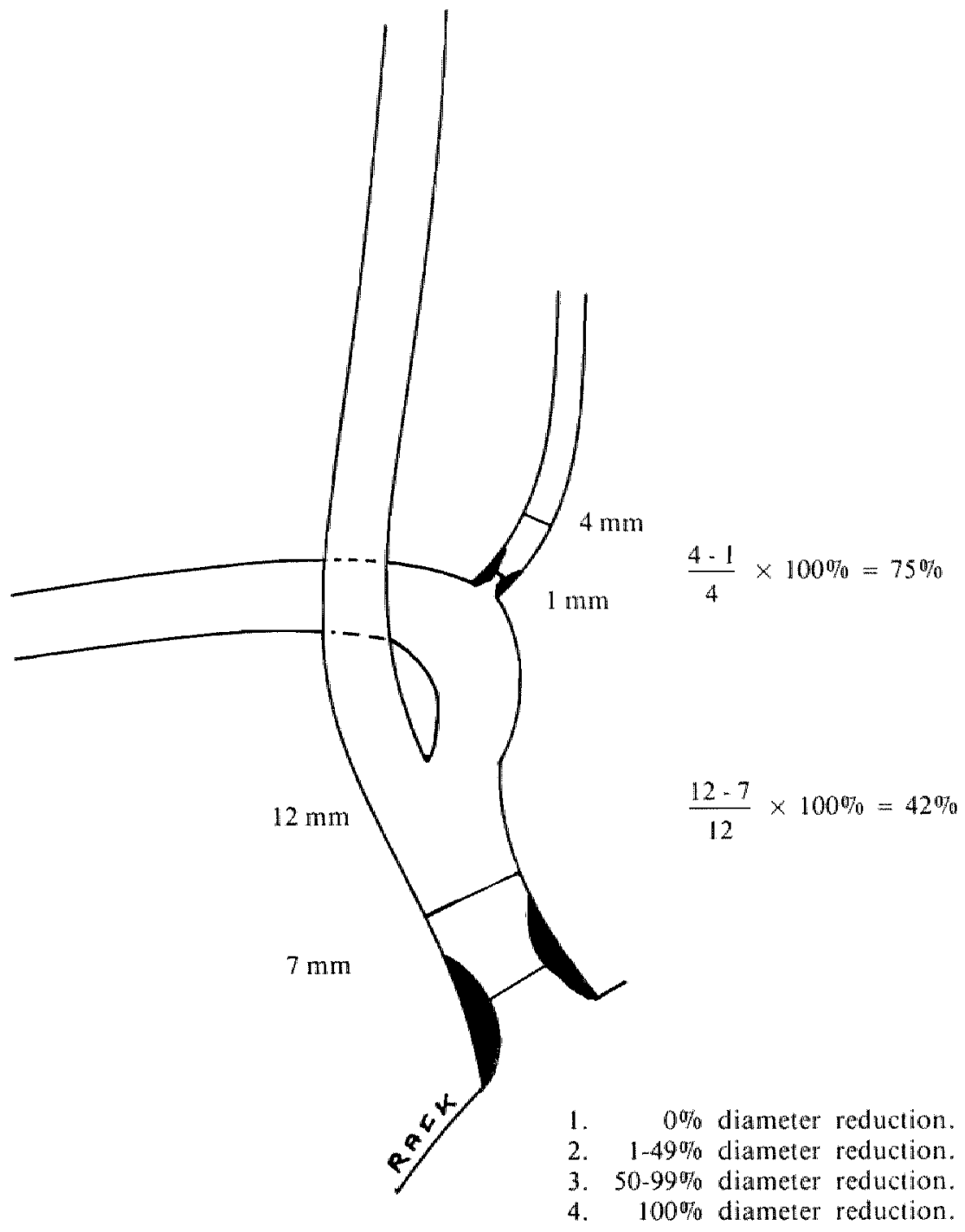

Figure 9.2/1

Assessment of degree of stenosis and arteriographic classification. 


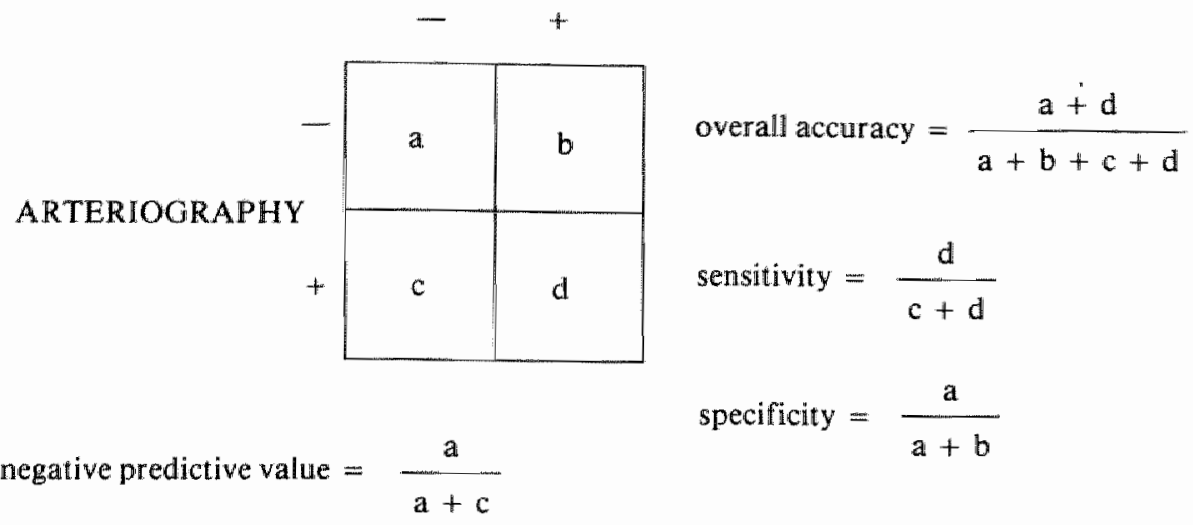

positive predictive value $=\frac{d}{b+d}$

Figure $9.2 / 2$

Decision matrix.

The sensitivity expresses the ability of a test to select the patients with the disease. It measures the fraction of patients with disease that will be selected by the test. The specificity is the ability to exclude the presence of the disease, as it measures the fraction of patients that will be correctly identified as having no significant disease. Since the sensitivity and the specificity are independent of the prevalence of the disease, in contrast to the overall diagnostic accuracy and the predictive values, the former are the better criteria to describe the accuracy of ultrasonic duplex scanning .

\subsection{THE FIRST STUDY (MAY 1981-DECEMBER 1982)}

During this period 900 ultrasonic duplex studies were performed on 850 patients. The most common reason for the investigation was the existence of symptoms of cerebrovascular insufficiency with or without carotid or subclavian bruits. Another important indication was the asymptomatic carotid bruit, especially in patients who were at risk for extracranial cerebrovascular disease. Some patients had undergone carotid endarterectomy before the duplex scanner was available and were sent to our laboratory for a non-invasive control study. 
Of these 850 patients, 65 men (mean age 63 years) and 17 women (mean age 58 years) underwent four-vessel arteriography. In one patient the arteriographic study was performed only on the left side. Seventy-nine patients were referred for the evaluation of suspected atherosclerotic disease of the extra- or intracranial cerebral vessels. In one patient the diagnosis was fibromuscular dysplasia, in one patient moyamoya disease, and in one patient Takayasu's disease.

\subsubsection{THE SUBCLAVIAN ARTERY}

Of the 163 subclavian arteries investigated in this study the results of contrast arteriography were of insufficient quality in eight cases and those of ultrasonic duplex scanning in another seven cases, giving 148 subclavian arteries available for comparison.

Table 9.3.1/1

Comparison of the results as obtained with duplex scanning and those obtained with contrast arteriography for the subclavian artery.
ARTERI-
DUPLEX
OGRAPHY

PERCENTAGE

OF STENOSIS

normal abnormal occlusion total

\begin{tabular}{lrccc}
\hline $0 \% 0$ & 112 & $3+3^{*}$ & & 118 \\
$1-49 \%$ & 9 & 6 & & 15 \\
$50-99 \%$ & 4 & 6 & 1 & 10 \\
$100 \%$ & & $4^{* *}$ & 1 & 5 \\
\hline total & 125 & 22 & 148 \\
\hline
\end{tabular}

* In three patients turbulent flow in the subclavian artery was probably the result of a stenosis of the innominate artery.

* This concerned four patients with an occlusion of the subclavian artery and a steal from the vertebral artery or thyrocervical trunk.

Table 9.3.1/1 shows the data of ultrasonic duplex scanning of the subclavian artery compared with contrast arteriography. Arteriographically there was a normal artery in 118 cases and 112 of these arteries were correctly identified with the duplex scanner. In six arteriographically normal arteries the duplex study suggested abnormalities. In three of these six cases the arte- 
riographic study showed a significant stenosis of the innominate artery. Therefore, the turbulent flow in the subclavian artery was probably the result of the stenosis of the innominate artery. In nine of the 15 arteries with a mild stenosis ( $1-49 \%$ diameter reduction) and in four of the ten arteries with a marked stenosis (50-99\% diameter reduction) the results of the duplex study were estimated as normal. In this study there were five occluded subclavian arteries. In three of these five arteries there was a subclavian steal from the ipsilateral vertebral artery. In another case there was a steal from the ipsilateral thyrocervical trunk. These four cases with a subclawian steal were correctly identified. The last occluded subclavian artery was also identified, but we failed to discern the ipsilateral vertebral artery.

\subsubsection{THE VERTEBRAL, ARTERY}

Of the 163 vertebral arteries included in this study a reliable radiological estimate of the origin was not possible in 46 cases. In 31 of the remaining 117 sides we could not identify the vertebral artery with the ultrasonic duplex scanner. In 14 instances we found a vertebral artery, but the results of duplex scanning did not permit a decision concerning the state of the ostium. Therefore, we could compare the data of contrast arteriography of the ostium of 72 vertebral arteries with the data of ultrasonic duplex scanning. The results of this comparison are shown in table 9.3.2/1.

Table 9.3.2/1

Comparison of the results as obtained with duplex scanning and those obtained with contrast arteriography for the vertebral artery (ostium).

ARTERI-

DUPLEX

OGRAPHY

PERCENTAGE

OF STENOSIS

normal abnormal

total

\begin{tabular}{lrrr}
\hline $0 \%$ & 45 & 4 & 49 \\
$1-49 \%$ & 3 & 8 & 11 \\
$50-99 \%$ & 2 & 10 & 12 \\
\hline total & 50 & 22 & 72 \\
\hline
\end{tabular}

Of the 49 vertebral arteries which showed no abnormalities at their origin, 45 were correctly identified. In four cases the duplex study suggested a stenosis. of the 23 arteries with an arteriographically determined obstructive lesion, 
the results of duplex scanning were in agreement in 13 cases. In eight cases there was a false positive and in only two cases a false negative result.

In this study we could not identify the vertrebral artery with the duplex scanner in $3 \rrbracket$ instances. The results of the contrast arteriographic examination of these 31 arteries are shown in table 9.3.2/2.

\section{Table 9.3.2/2}

Results of the contrast arteriographic study of the ostium of 31 vertebral arteries which were not found by ultrasonic duplex scanning.

\begin{tabular}{|c|c|c|c|c|c|c|}
\hline ANGIO & $\begin{array}{c}\text { marked } \\
\text { hypoplasia }\end{array}$ & agenesis & normal & $1-49 \%$ & $50-990 \%$ & occlusion \\
\hline & 3 & 1 & $10+1 *$ & 5 & 3 & $7+1 * *$ \\
\hline
\end{tabular}

* This concerned one patient with an abnormal origin of the left vertebral artery from the aortic arch.

This concerned one patient with an occluded left subclavian and left vertebral artery. There was a steal from the left thyrocervical trunk. This was interpreted as a subclavian steal from the left vertebral artery.

In one case the artery remained invisible during the whole radiological study and the conclusion of the radiologists was a congenital absence. Three times there was a marked hypoplasia. Of the remaining 27 arteries the arteriogram was completely normal in 11 cases. In five arteries there was a mild stenosis ( $1-49 \%$ diameter reduction) and in three arteries a marked stenosis $(50-99 \%$ diameter reduction) at the site of the ostium. In eight cases the radiological study showed an occlusion of the vertebral artery. In seven of these eight patients we were unable to find the vertebral artery with the duplex scanner as well. In the last patient we found an occluded left subclavian artery and distal to this occlusion a vessel with reversed fllow. This was interpreted as a subclavian steal from the left vertebral artery. The arteriographic study, however, showed an occlusion of the left subclavian and left vertebral arteries with a steal from the thyrocervical trunk.

\subsubsection{DISCUSSION OF THE RESULTS OF THE FIRST STUDY}

\section{subclavian artery}

The results of the first study show that ultrasonic duplex scanning of the subclavian artery is a reliable test (tables $9.3 .1 / 1$ and $9.3 .3 / 1$ ). For the detection of an obstructive lesion with a diameter reduction of $50 \%$ or more, or 
occlusions a sensitivity of $0.73(11 / 15)$ and a specificity of $0.91(121 / 133)$ was found. There was a false positive result in only six of the 118 angiographically normal subclavian arteries and in three of these six arteries the turbulent flow in the subclavian artery was probably the result of a marked stenosis of the innominate artery. The predictive value of a normal test was remarkably high $0.97(121 / 125)$, whereas the predictive value of a positive test was very low $(0.48,11 / 23)$. The former was of course biased by the relatively high number of subclavian arteries without significant disease (133). Nevertheless, in four arteries with a stenosis of $50 \%$ or more no disturbances were found in the sonagrams with the duplex scanner. The reason for this was the fact that in this study we could not perform a Doppler measurement in the most proximal part of the subclavian artery, even on the right side. This was an extra motivation to image the innominate bifurcation in the next study.

However, the major problem was the relative high number of false positive results. In vessels with minor wall irregularities it was difficult to distinguish the flow disturbances as induced by these lesions from the disturbances occurring in normal vessels because of an excentrically placed sample volume.

Table $9.3 .3 / 1$

The sensitivity, specificity, positive and negative predictive values, and the overall accuracy for identifying a stenosis of $50 \%$ or more in the subclavian and vertebral (ostium) arteries.

\begin{tabular}{lccccc} 
VESSEL & sensitivity & specificity & $\begin{array}{c}\text { positive } \\
\text { predictive } \\
\text { value }\end{array}$ & $\begin{array}{c}\text { negative } \\
\text { predictive } \\
\text { value }\end{array}$ & $\begin{array}{c}\text { overall } \\
\text { accuracy }\end{array}$ \\
\hline $\begin{array}{l}\text { subclavian } \\
\text { artery }\end{array}$ & 0.73 & 0.91 & 0.48 & 0.97 & 0.89 \\
$\begin{array}{l}\text { vertebral } \\
\text { artery }\end{array}$ & 0.83 & 0.80 & 0.45 & 0.96 & 0.81 \\
\hline
\end{tabular}

\section{vertebral artery}

In this study we investigated the vertebral artery by visualization of the subclavian artery. This vessel was then followed in a distal direction until the vertebral artery was found. One of the major problems when using this technique was to ensure that the signals recorded indeed originated from the vertebral artery. 
We failed to find a vertebral artery in 31 instances and only eight of these 31 arteries were occluded. In one of these eight patients we mistakenly recorded signals from the thyrocervical trunk which might also have happened in other cases. In contrast to ultrasonic duplex scanning of the carotid bifurcation, it is usually not possible to image a vertebral artery in the event of an occlusion. An important reason for this may be the limited capabilities of the available scan heads.

In 14 cases it was impossible to make a decision about the patency of the ostium of the vertebral artery with the duplex scanner. In these cases the artery could easily be found and scanning of a more distal part of the vessel presented no problem, but the ostium was located too deeply.

Similar to the subclavian artery, classification of the degree of stenosis of the vertebral artery posed a problem. Relative to the diameter of the vertebral artery the sample volume of the scan head was too large. The detection of minor obstructive lesions by use of spectral broadening was not possible. However, ultrasonic duplex scanning appeared to be a reliable test for the detection of an obstructive lesion at the site of the ostium of the vertebral artery with a diameter reduction of $50 \%$ or more (tables $9.3 .2 / 1$ and $9.3 .3 / 1$ ). The sensitivity and the specificity were respectively $0.83(10 / 12)$ and 0.80 $(48 / 60)$. In only two arteries with a haemodynamically significant lesion the duplex scanning gave a false negative outcome, resulting in a negative predictive value of $0.96(48 / 50)$. The major problem was the low positive predictive value of the test $(0.45,10 / 22)$. A reasonable explanation for this relative high number of false positive results was not known during this part of the study.

\subsection{THE SECOND STUDY (DECEMBER \982-OCTOBER 1984)}

During this period ultrasonic duplex scanning of the extracranial cerebral vessels became of increasing interest to neurologists, neurosurgeons, and vascular surgeons. Physicians collaborating with the St. Antonius Hospital, Nieuwegein, The Netherlands, were informed about the facilities and sent their patients to our laboratory.

Between December 1982 and October 1984, 1870 duplex studies were performed on 1122 patients. Most patients were referred to our department by wascular surgeons, neurologists, neurosurgeons, or cardiologists. As in the first study, the most important reason for ultrasonic duplex scanning was the existence of symptoms of cerebrovascular insufficiency of the anterior or posterior circulation, or an asymptomatic carotid bruit in patients at risk for extracranial cerebrovascular disease. During the time of the second study, about $40 \%$ of the patients were included in prospective follow-up studies for a non-haemodynamically significant carotid lesion, an asymptomatic carotid bruit, carotid or vertebral endarterectomy or extra-intracranial bypass surgery. 
Of these 1122 patients 82 men (mean age 58 years) and 41 women (mean age 59 years) underwent multiplanar contrast arteriography. In one patient the arteriographic study was only performed on the right side. One hundred and nineteen patients were referred for the evaluation of suspected atherosclerosis of the extra- or intracranial cerebral vessels. In three patients the diagnosis was fibromuscular dysplasia and in one patient Takayasu's disease.

\subsubsection{THE INNOMINATE ARTERY}

In 17 of the 123 patients investigated in the second study the innominate artery was located too deeply and an estimate of the patency of the artery with the duplex scanner was not possible. In 11 patients the results of contrast arteriography were of insufficient quality and in another ten patients arch aortography was not performed. Two patients showed an abnormal origin of the right subclavian artery directly from the aorta distal to the origin of the left subclavian artery. Therefore, we could compare the data of ultrasonic duplex scanning of the innominate artery with the data of contrast arteriography in 83 patients.

The results of this comparison are shown in table 9.4.1/1.

Table $9.4 .1 / 1$

Comparison of the results as obtained with duplex scanning and those obtained with contrast arteriography for the innominate artery.

ARTERI-

OGRAPHY

DUPLEX

PERCENTAGE

OF STENOSIS

normal abnormal occlusion

total

\begin{tabular}{lllrr}
\hline $0 \%$ & 66 & 1 & & 67 \\
$1-49 \%$ & 13 & 2 & 1 & 2 \\
$50-99 \%$ & & & 1 \\
$100 \%$ & 79 & 3 & 1 & 83 \\
\hline total & 79 & & 1 \\
\hline
\end{tabular}

In 66 of the 67 patients with a normal innominate artery and in all 13 patients with a mild stenosis (1-49\% diameter reduction) the data of ultrasonic duplex scanning was in agreement with the data of contrast arteriography. There was a false positive result in only one of these 80 patients. Both arteries with a 
marked stenosis (50-99\% diameter reduction) were also correctly identified. In this study there was one patient with an occlusion of the innominate artery. In this patient no velocity of flow was found in the distal part of the innominate artery and the low and abnormal damped Doppler signals in the right common carotid and subclavian arteries with a complete steal from the right vertebrall artery were interpreted as signs of a severe lesion of the innominate artery.

\subsubsection{THE SUBCLAVIAN ARTERY}

As the anatomy of the first segment of the right subclavian artery differs considerably from the left, we decided to separate the results of ultrasonic duplex scanning of these two vessels.

On the right side the results of the radiological study were of insufficient quality in 19 patients and in another seven patients arch aortography was not performed. In one patient the results of the duplex study were unreliable because of a significant stenosis of the innominate artery. Thus 96 right subclavian arteries were available for comparison and the results are presented in table $9.4 .2 / 1$.

Table $9.4 .2 / 1$

Comparison of the results as obtained with duplex scanning and those obtained with contrast arteriography for the right subclavian artery.

ARTERI-

OGRAPHY

DUPLEX

PERCENTAGE

OF STENOSIS

normal

abnormal

occlusion

total

$\begin{array}{lrrr}0 \% & 81 & & 81 \\ 1-49 \% & 7 & 1 & 8 \\ 50-99 \% & 4 & 3 & 7 \\ 100 \% & & & \end{array}$

total

92

4

96

All 81 angiographically normal right subclavian arteries and seven of the eight cases with a mild stenosis (1-49\% diameter reduction) were correctly identified. In only one of these 89 patients the duplex study suggested a 
significant stenosis. However, of the seven right subclavian arteries with a marked stenosis (50-99\% diameter reduction) on arteriography only three cases were correctly identified. In four cases the results of the duplex study were estimated as normal. In this part of the study there was no occlusion of a right subclavian artery.

For the left subclavian artery the results of the contrast arteriographic study were of insufficient quality in 18 patients and arch aortography was not performed in another 11 patients, giving 94 left subclavian arteries available for comparison.

Table $9.4 .2 / 2$

Comparison of the results as obtained with duplex scanning and those obtained with contrast arteriography for the left subclavian artery.

\section{ARTERI-}

OGRAPHY

\section{DUPLEX}

\section{PERCENTAGE}

\begin{tabular}{lcccc} 
OF STENOSIS & normal & abnormal & occlusion & total \\
\hline $0 \%$ \% & 75 & 2 & & 75 \\
$1-49 \%$ & 9 & 5 & 2 & 11 \\
$50-99 \%$ & 1 & & 2 & 2 \\
$100 \%$ & & 7 & 2 & 94 \\
\hline total & 85 & & 2 & \\
\hline
\end{tabular}

Table 9.4.2/2 shows the data of ultrasconic duplex scanning of the left subclavian artery compared with contrast arteriography. All 75 arteriographically normal left subclavian arteries and nine of the 11 cases with a mild stenosis (1-49\% diameter reduction) were correctly identified. In only two cases the degree of stenosis was overestimated with the duplex scanner. In six patients the radiological study showed a marked stenosis $(50-99 \%$ diameter reduction) and five of these six lesions were correctly identified. In only one artery was there a false negative result. In this part of the study there were two occluded left subclavian arteries. In one of these patients the contrast arteriographic study showed a steal from the left vertebral artery and in the remaining patient a steal from the left vertebral artery and thyrocervical trunk as well. These two cases with a subclavian steal syndrome were correctly identified with the duplex scanner. 


\subsubsection{THE PREVERTEBRAL SEGMENT OF THE VERTEBRAL ARTERY}

As described in section 8.1.4, we routinely investigated the prevertebral segment of the vertebral artery from a distal to a proximal position during the period of the second study. The vertebral artery was first visualized at the Chassaignac tubercle, just before it enters its bony canal. Thereafter it was followed in a proximal direction as far as its origin from the subclavian artery. In this part of the study a decision about the state of the prevertebral segment of the vertebral artery was only made when this segment could continuously be followed by the technician along its whole course. The data of ultrasonic duplex scanning of the prevertebral segment of the vertebral artery was divided into two groups: 1. the data concerning the state of the ostium, and 2 . the data concerning the remaining part of the prevertebral segment.

Of the 245 vertebral arteries investigated between December 1982 and $\mathrm{OC}-$ tober 1984 a reliable radiological estimate of the origin was not possible in 36 cases. In 43 instances we found a vertebral artery with the duplex scanner, but the results of the non-invasive study did not permit a decision concerning the state of the ostium. In 22 cases the results were discarded because of the insufficient quality of both investigations. In 24 of the remaining 144 sides we could not identify the vertebral artery with the duplex scanner.

Therefore, the data as obtained with contrast arteriography could be compared with the data as obtained with ultrasonic duplex scanning in 120 vertebral arteries. The results of this comparison are presented in table 9.4.3/1.

Table $9.4 .3 / 1$

Comparison of the results as obtained with duplex scanning and those obtained with contrast arteriography for the vertebral artery (ostium).

ARTERI-

OGRAPHY

DUPLEX

PERCENTAGE OF STENOSIS

normal

abnormal

total

\begin{tabular}{lrrr}
\hline $0 \%$ & 71 & 2 & 73 \\
$1-49 \%$ & 11 & 9 & 20 \\
$50-99 \%$ & 5 & 22 & 27 \\
\hline total & 87 & 33 & 120 \\
\hline
\end{tabular}


Of the 73 vertebral arteries, which showed no abnormalities at their origin, 71 were correctly identified with the duplex scanner. In only two cases the duplex study suggested a stenosis. In 11 of the 20 arteries with a mild stenosis ( $1-49 \%$ diameter reduction) and in 22 of the 27 arteries with a marked stenosis (50-99\% diameter reduction) the data of ultrasonic duplex scanning was in agreement with the data of contrast arteriography. In nine cases there was a false positive and in five cases a false negative result.

In this study we could not identify the vertebral artery with the duplex scanner in 24 instances. The results of contrast arteriography of these 24 arteries are given in table 9.4.3/2.

Table $9.4 .3 / 2$

Results of the contrast arteriographic study of the ostium of 24 vertebral arteries which were not found by ultrasonic duplex scanning.

\begin{tabular}{|c|c|c|c|c|c|c|}
\hline ANGIIO & $\begin{array}{c}\text { marked } \\
\text { hypoplasia }\end{array}$ & agenesis & $\begin{array}{c}\text { normal } \\
2^{*}\end{array}$ & $1-490 \%$ & $\begin{array}{c}50-99 \% \\
2^{* * *}\end{array}$ & $\begin{array}{c}\text { occlusion } \\
20\end{array}$ \\
\hline
\end{tabular}

This concerned one right vertebral artery of a relatively small caliber in a patient with obesity

* This concerned one patient with a tight stenosis of the ostium of the left vertebral artery with very low flow during the arteriographic examination.

In two cases the arteriogram was completely normal and in two other cases there was a stenosis with a diameter reduction of $50 \%$ or more at the site of the ostium. However, in 20 cases contrast arteriography showed an occlusion of the vertebral artery.

According to our experience, significant atherosclerotic lesions in the remaining part of the prevertebral segment of the vertebral artery were very rare. In only two patients ultrasonic duplex scanning showed a stenosis with a diameter reduction of $50 \%$ or more in the prevertebral segment of the vertebral artery $1-2 \mathrm{~cm}$ distal to the origin. In one of these patients arteriography showed a stenosis with a diameter reduction of $50 \%$ at that location. In the remaining patient the quality of the arteriographic study did not permit a decision about the status of the prevertebral segment of the vertebral artery. In a third patient arteriography of the right vertebral artery showed a stenosis with a diameter reduction of $30-40 \% 02-3 \mathrm{~cm}$ distal to the ostium. In this patient the non-invasive investigation of the prevertebral segment of the right vertebral artery was well performed along its whole length. However, no abnormalities were found in this segment of the vertebral artery with the 
duplex scanner. In two other patients arteriography revealed a stenosis with a diameter reduction of about $30 \%$ in the prevertebral segment of the right vertebral artery just proximal to the transverse process of the sixth cervical vertebra. In one of these patients the sonagrams recorded at this location were normal. In the remaining patient they were disturbed and pointed to a significant stenosis.

\subsubsection{THE CERVICAL SEGMENT OF THE VERTEBRAL ARTERY}

As mentioned in section 9.1, we also investigated the cervical segment of the vertebral artery during the period of the second study. However, for time saving reasons, the investigation of the cervical segment was only performed if the patient had a history suspicious of vertebrobasilar arterial insufficiency or if the investigation of this segment of the vertebral artery was emphatically requested by the referring physician.

In the first instance we attempted to use the data of intravenous digital subtraction angiography for comparison with the data as obtained with ultrasonic duplex scanning. Usually, displacement of the vertebral arteries by osteo-arthritic spurs of the cervical vertebrae was well demonstrated by this technique. However, pictures of the masks were not routinely made by the department of roentgenology of our hospital and, therefore, an exact location of the displacement of the vertebral artery was impracticable in most patients. Moreover, in our opinion intravenous digital subtraction angiography is inadequate when making an estimation of the percentage of stenosis of the vertebral arteries. Consequently, a satisfactory differentiation between displacement of the vertebral artery with or without localized stenosis and atherosclerotic obstructive disease in the cervical segment of the vertebral artery without displacement of the artery by osteo-arthritic spurs could not be expected by using this technique. Therefore, for the purpose of this study we only used the data obtained with conventional arteriography or intra-arterial digital substraction angiography if the unsubtracted arteriograms or the masks were available for comparison.

During the second study a complete ultrasonic investigation of the cervical segment of the vertebral artery was performed in $70 \%(790 / 1122)$ of the patients. In 97 of the 123 patients ( $79 \%$ ) included in the second study both the arteriographic examination and ultrasonic duplex scanning permitted a decision about the state of the cervical segment of at least one vertebrall artery. In 87 of these 97 patients $(90 \%)$ no abnormalities were found in the cervical segment of both vertebral arteries either with the duplex scanner or by arteriography. In 10 patients $(10 \%)$ abnormalities such as displacement of the vertebral artery by osteo-arthritic spurs or abnormaly high and turbulent Doppler signals pointing to a significant stenosis were found in the cervical 
segment of at least one vertebral artery with the duplex scanner. The data of the arteriographic and duplex examinations of these ten patients is described in table $9.4 .4 / 1$.

\section{Table $9.4 .4 / 1$}

The 10 patients in whom ultrasonic duplex scanning of the vertebral artery showed abnormalities in the cervical segment as compared with contrast arteriography.

\section{CASE SEX AGE RIGHT VERTEBRAL LEFT VERTEBRAL}

1. 54 duplex: normal ostium, abormal Doppler signals at C6-C5 without coiling.

angio: normal ostium, a stenosis with a diameter reduction of $50-75 \%$ without coiling at C6-CS. duplex: high and turbulent Doppler signals at the site of the ostium, otherwise a normal vertebral artery.

angio: a stenosis with a diameter reduction of $80 \%$ at the site of the ostium, otherwise a normal vertebral artery.

2. M 52 duplex: normal ostium, coiling at $\mathrm{C} 5 \mathrm{C} 4$, no signs of a stenosis at this location.

duplex: no decision about the state of the ostium, otherwise a normal vertebral artery.

angio: a stenosis with a diameter reduction of $33 \%$ at the ostium, coiling at C5. angio: a normal vertebral artery.

C4, no signs of a stenosis at this location.

3. $\mathrm{F} 63$ duplex: normal ostium, coiling at C5-C4, C4-C3, and $\mathrm{C} 3-\mathrm{C} 2$ without signs of a stenosis at these locations.

angio: normal ostium, coiling at $\mathrm{C} 5-\mathrm{C} 4, \mathrm{C} 4-\mathrm{C} 3$, and $\mathrm{C} 3-\mathrm{C} 2$, no signs of a stenosis at these locations. duplex: no decision about the state of the ostium, coiling at $\mathrm{C} 6 \times \mathrm{C} 5, \mathrm{C5}-\mathrm{C} 4$, C4-C3 without signs of a stenosis at these locations.

angio: normal ostium, coiling at $\mathrm{C} 6=\mathrm{C} 5, \mathrm{C} 5-\mathrm{C} 4$, and $\mathrm{C} 4-\mathrm{C} 3$, no signs of a stenosis at these locations.

The results of ultrasonic duplex scanning and arteriography of this patient are illustrated in the figures $9.4 .4 / 1$ and $9.4 .4 / 2$. 
4. M 65 duplex: high and turbulent duplex: normal ostium, Doppler signals at the site of coiling at C6-C5 without the ostium, coiling at $\mathrm{C4}-\mathrm{C} 3$ signs of an stenosis at this without signs of a stenosis at location. this location.

angio: a stenosis with a diameter reduction of $75 \%$ at the site of the ostium, coiling at $\mathrm{C} 4-\mathrm{C} 3$ without a sitenosis at this location.

angio: no decision about the state of the ostium, coiling at $\mathrm{C6}-\mathrm{C} 5$ without a stenosis at this location.

5. $\mathbb{F}$

63 duplex: normal ostium, high and turbulent Doppler signals just proximal to $\mathrm{C} 6$ and at $\mathrm{C} 4-\mathrm{C} 3$ without coiling.

angio: three obstructive lesions with a diameter reduction of:

1. $33 \%$ at the site of ostium, 2. $30 \%$ just proximal to $\mathrm{C} 6$ and, $3.60 \%$ at $\mathrm{C} 4$. angio: a proximal occlusion with distal collateral filling via the thyrocervical trunk.
6.

F

54

duplex: no decision about the state of the ostium, coiling at $\mathrm{C} 5-\mathrm{C} 4$ without signs of a stenosis at this location.

angio: normal ostium, coiling at $\mathrm{C} 5-\mathrm{C} 4$ without stenosis at this location. duplex: no decision about the state of the ostium, coiling at $\mathrm{C} 5-\mathrm{C} 4$ without signs of a stenosis at this location.

angio: normal ostium, coiling at $\mathrm{C} 5 \mathrm{C} 4$ without stenosis at this location. 
7. M 45 duplex: normal ostium, coiling at C6-C5 without duplex: normal vertebral signs of a stenosis at this artery. location.

angio: normal ostium, coiling at C6-C5 without angio: no decision about the stenosis at this location. state of the ostium, otherwise a normal vertebral artery.

8. M 64 duplex: a high-flow state duplex: no decision about and extra increased and the state of the ostium, turbulent Doppler signals at the site of the ostium and at otherwise a hypoplastic C6-C5 without coiling. vertebral artery.

angio: a stenosis with a diameter reduction of $20 \%$ at the site of the ostium and angio: no arteriography of the left vertebral artery of $33 \%$ at $\mathrm{C} 6$, no coiling. available.

The same patient as case 4 of table $9.4 .8 / 2$

9.

M

duplex: a bigh-flow state, duplex: a proximal therefore no decision about the state of the ostum, extra occlusion, low Doppler increased Doppler signals at C6-C5 without coiling. signals more distally.

angio: normal ostium, a angio: a proximal occlusion stenosis with a diameter with distal collateral filling reduction of $33 \%$ at $\mathrm{C} 6-\mathrm{CS}$ via the thyrocervical trunk. without coiling. 
10. M 63

duplex: normal ostium, coiling at $\mathrm{C} 5-\mathrm{C} 4$ without signs of a stenosis at this location.

angio: no decision about the state of the ostium and a stenosis with a diameter reduction of 30-40\% without coiling at $\mathrm{C5}-\mathrm{C} 4$. duplex: no decision about the state of the ostium, otherwise a normal vertebral artery.

angio: no decision about the state of the ostium, otherwise a normal vertebral artery.

A displacement of the vertebral artery in its cervical segment without signs of a stenosis in the displaced segments was found with the duplex scanner in five patients (case 2,3,4,6 and 7). In two of these patients the displacement of the vertebral artery only appeared on the right side and in three patients bilaterally. In one patient (case 3) the displacement appeared on both sides at three levels. In the remaining four patients the displacement was found at only one level. In all five patients a remarkable agreement existed between the data of arteriography and those obtained with ultrasonic duplex scanning.

In four patients (case 1,5,8 and 9) abnormally high and turbulent Doppler signals pointing to a significant stenosis were found in the cervical segment of the vertebral artery with the duplex scanner. However, the duplex examination did not reveal any displacement of the vertebral artery. In these four cases the conclusion of the duplex examination of the cervical segment of the vertebral artery was: an obstructive lesion, probably with a diameter reduction of $50 \%$ or more. All four lesions were located on the right side. In two of these four patients (case 8 and 9) the duplex examination showed a high-flow state in the right vertebral artery. In these two patients arteriography revealed a stenosis with a diameter reduction of $33 \%$. In the remaining i wo patien is (case 1 and 5) there was a stenosis on arteriography with a diameter reduction of $50-75$ and $60 \%$, respectively.

In the last puirent (case 10) there is some disagreement between the data of ultrasonic duplex scanning and that obtained by contrast arteriography. The former showed minor coilling of the vertebral artery at C5-C4 with normal Doppler signals at this level, while the latter revealed a stenosis with a diameter reduction of $30-40 \%$ at $\mathrm{C} 5-\mathrm{C} 4$ without coiling of the artery, 


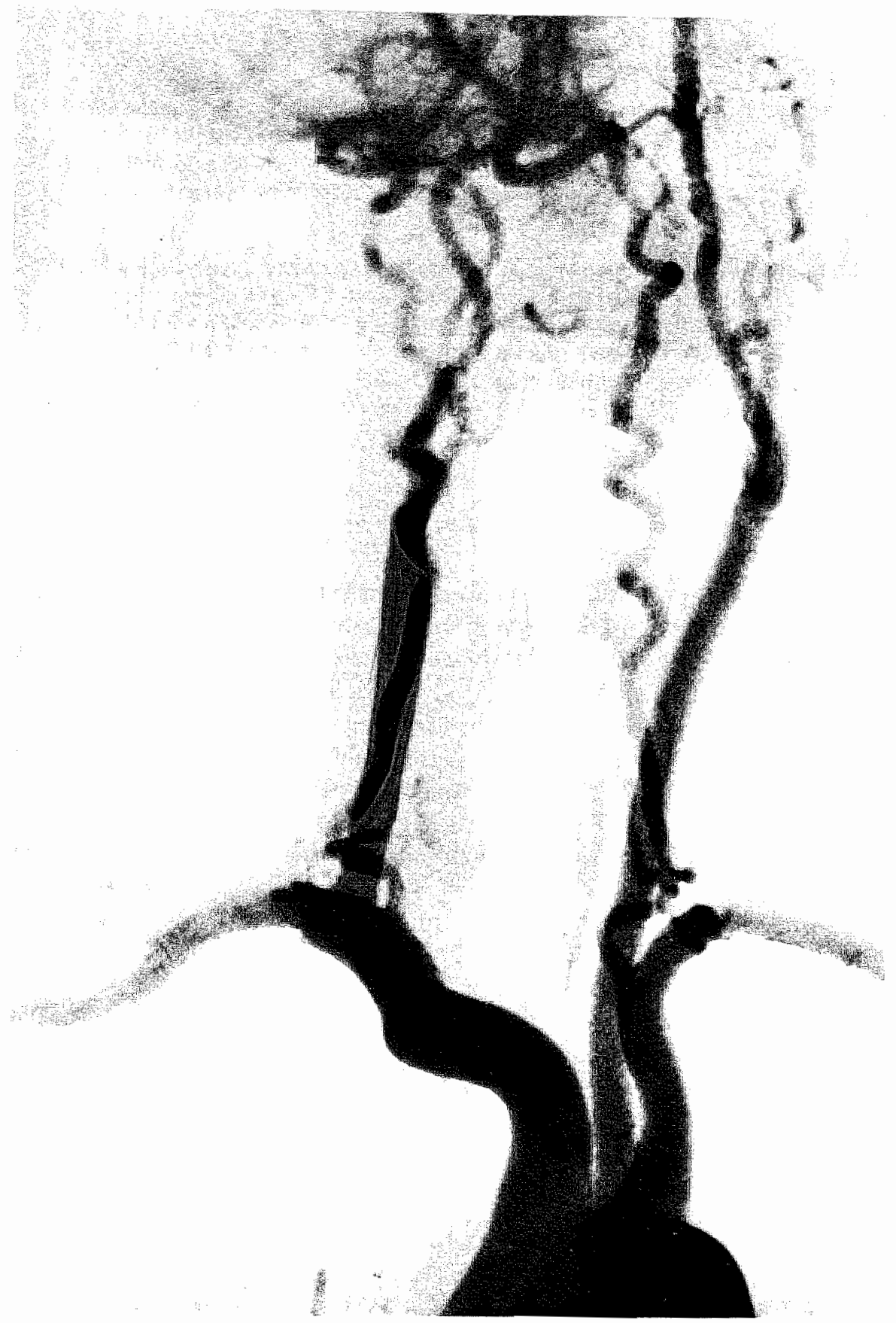

Figure 9.4.4/1

The aortogram of case 3 of table 9.4.4/1. Both vertebral arteries were pushed aside at three levels by osteo-arthritic spurs. No localized stenoses were demonstrated in the cervical segment of the vertebral arteries. 

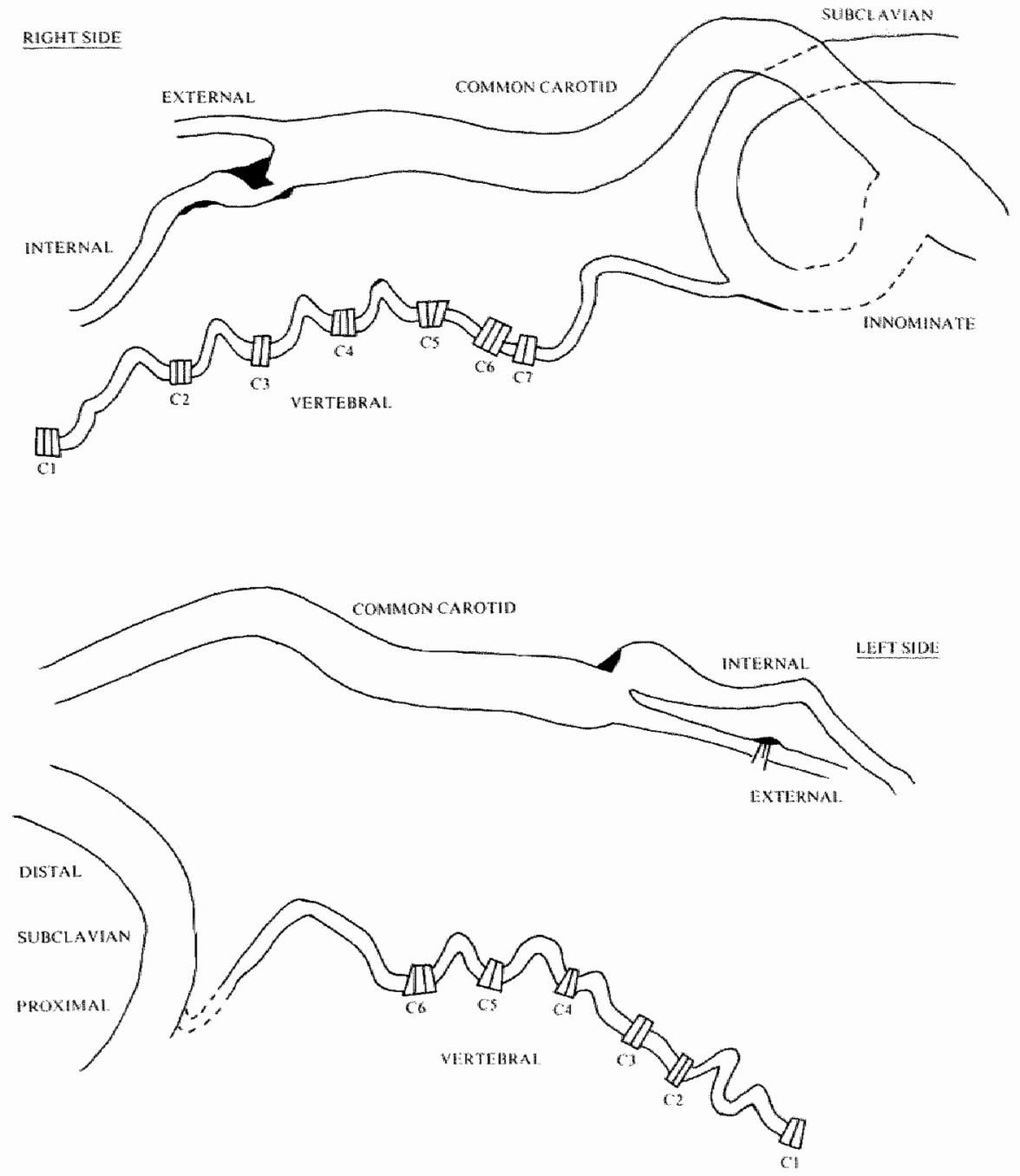

Figure 9.4.4/2

The same patient as in fig. 9.4.4/1. Ultrasonic duplex scanning demonstrated displacement of the vertebral arteries at the same level as contrast arteriography. No stenosis was found in the cervical segment of both vertebral arteries. Apart from this, the right vertebral artery enters its bony canal at the transverse process of the seventh cervical vertebra. 
An investigation of the atlantic segment of the vertebral artery was not possible with the duplex scanner. In order to evaluate rotational compression of the vertebral artery at the level of the atlanto-axial articulation, the most distal part of the cervical segment of the vertebral artery between the transverse processes of the second and first cervical vertebrae was investigated during the period of the second study in nearly every patient in whom the cervical segment was studied. In spite of the fact that during ultrasonic duplex scanning of the distal part of the cervical segment of the vertebral artery the head of the patient was turned to the contralateral side for about 30 to 45 degrees, the Doppler signals recorded in this segment of the vertebrall artery were normal in most of the patients. In some patients with normal Doppler signals more proximal in the cervical segment of the vertebral artery, high and turbulent velocities were found at the level of the atlanto-axial articulation. However, the angle of insonation was relatively acute in most of these cases. Therefore, we did not make a definitive decision upon the status of this segment of the vertebral artery in these cases. In one patient with bilaterally occluded common carotid arteries and significant stenoses at the site of the ostium of both vertebral arteries, high and turbulent Doppler signals were also found in both vertebral arteries at the level of the atlanto-axial articulation. After carotid and vertebral endarterectomy on the right side, the sonagrams recorded in the most distal part of the cervical segment of the vertebral arteries were normalized.

No lesions were demonstrated in the most distal part of the cervical segment and atlantic segment of the vertebral artery in any of the patients who underwent contrast arteriography.

\subsubsection{THE SIGNIFICANCE OF ULTRASONIC DUPLEX SCANNING IN CASES OF SEVERE, OBSTRUCTIVE DISEASE OF THE INTRADURAL SEGMENT OF THE VERTEBRAL ARTERY}

During the second study, contrast arteriography revealed an intracranially located stenosis with a diameter reduction of $50 \%$ or more or an intracranially located occlusion of the vertebral artery in seven of 123 patients $(5.7 \%)$. The results of both contrast arteriography and ultrasonic duplex scanning of the vertebral arteries of these seven patients are shown in table 9.4.6/1. 


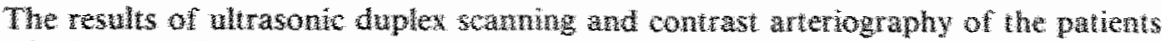

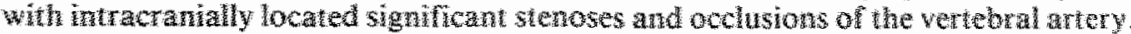

\section{CASE SEX AGE RICHT VERTERRAL LETTVERTEBRAL}

1. F 59 duptex: nomal wertebral
artery, no bunted sigmals.

duplex: butured signals pointing to a significant tesion in the intradural segnent.

angio: normal ostima stemosis with a diameter reduction of 80 me in the

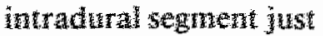
proximal wo the origin of the PMCA.

ang: 10 : stenosis wh diameter reduction of $70 \%$ at the sute of the ostum and an occlusion just proximat to the orign of the PICA.

The same paturnt as case of the $9.4 .8 / 3$

\begin{tabular}{|c|c|c|c|}
\hline$F$ & 66 & $\begin{array}{l}\text { duplex: normal vertebral } \\
\text { artery. }\end{array}$ & $\begin{array}{l}\text { duplex. normal vertebral } \\
\text { antery. }\end{array}$ \\
\hline & & $\begin{array}{l}\text { angio: a stenosis wha a } \\
\text { dymetar reduction of } 50 \text { - } \\
\text { 6oto just prowimal to the } \\
\text { ancton. }\end{array}$ & $\begin{array}{l}\text { dngio: a stenosis with } \\
\text { diameter reduction of } 80 \\
90 \% \text { just proximal to the } \\
\text { junction. }\end{array}$ \\
\hline
\end{tabular}

3. $\mathrm{M} 67$ duplex: bunted signals pointing to a significan leston the thradural duplex: normal vertebrat arery. segment.

angio: an ocelusion the intradural segment.

4. M 54 duplex: normal vertebral artery with low velocities during the entire cardiac cycle.

angio: an occlusion just distal to the origin of the PICA. angino: nomal vertebral artery.

duplex: biunted signals pointing to a significant lesion in the intradural segment.

angio: occlusion just proximal to the origin of the PICA. 
5. F 72 duplex: normal vertebral
artery.

angio: normal vertebral artery. duplex: normal vertebral artery with low velocities during the entire cardiac cycle.

angio: an occlusion just distal to the origin of the PICA.

\section{M 67 duplex. normal vertebral artery.}

angio: normal vertebral artery. duplex: vertebral artery with a small caliber with low wellocities during the entire cardiac cycle.

angio: vertebral artery with a small caliber which only perfused the PICA (congenital anomaly).
7. $\mathrm{F}$

52 duplex: normal vertebral
artery.

angio: normal vertebral artery. duplex: blunted signals pointing to a significant lesion in the intradural segment.

angio: an occlusion just distal to the origin of the PICA.

In four patients (case 3,5,6 and 7) the lesion was located unilaterally and in three patients (case 1,2 and 4) bilaterally. In three vertebral arteries (case 1: right vertebral artery and case 2 ; both vertebral arteries) with a stenosis with a diameter reduction of $50 \%$ or more in the intradural segment, the Doppler signals recorded more proximally in these vertebral arteries were quite normal. Case 6 was a patient with a very exceptional congenital anomaly of the cerebral arteries. On the left side there was a congenital connection between the external carotid and basilar arteries. The left vertebral artery arose from the left subclavian artery as normal, was of a small caliber, and only intracranially perfused the left posterior inferior cerebellar artery. In this relatively small vertebral artery low flow velocities with forward flow during the entire cardiac cycle were recorded. Two other vertebral arteries (case 4 and 5 : left vertebral artery) showed the same kind of sonagrams as recorded in the left 
vertebral artery of case 6 . In these two vertebral arteries with a normal caliber the occlusion was located just distal to the origin of the left posterior inferior cerebellar artery. In the remaining four vertebral arteries (case 3 : right vertebral artery and case 1, 4, and 7: left vertebral arteries) ultrasonic duplex scanning showed blunted Doppler signals pointing to a significant lesion more distally located in these arteries. In two of these four cases (case 1 and 4) the occlusion of the vertebral artery was located proximal and in one case (case 7) just distal to the origin of the posterior inferior cerebellar artery. In the remaining patient (case 3) contrast arteriography did not allow an exact location of the occlusion.

\subsubsection{THE SUBCLAVIAN AND INNOMINATE STEAL SYNDROMES}

Between December 1982 and October 1984 the extracranial cerebral arteries were non-invasively investigated by ultrasonic duplex scanning in 1122 patients. In 123 of these patients a comparion was possible between the data of ultrasonic duplex scanning of the innominate-subclavian-vertebral arterial system and those of contrast arteriography. In eight of these 123 patients ultrasonic duplex scanning found a subclavian or innominate steal syndrome. More detailed information about these steal syndromes is shown in table 9.4.7/1.

Table 9.4.7/1

The eight subclavian and innominate steal syndromes found in the patients who were included in the second study.

RIGHT SIDE

LEFT SIDE

subclavian steal syndrome

latent : : 0

transient : :0

permanent $: 1$

total : 1 subclavian steal syndrome

latent $\quad: 0$

transient $: 2$

permanent : 3

total : 5

innominate steal syndrome

latent : : 0

transient : 0

permanent : 2

total : 2

Of these 123 patients a permanent subclavian steal syndrome on the right side was found with the duplex scanner in one patient. In this patient contrast arteriography showed a stenosis with a diameter reduction of $75 \%$ in the right 
subclavian artery. In two patients there was a transient subclavian steal syndrome on the left side with an arteriographically confirmed occlusion of the left subclavian artery. In another three patients ultrasonic duplex scanning found a permanent subclavian steall syndrome on the left side. In one of these three patients contrast arteriography showed an occlusion of the left subclavian artery and in the remaining two patients a stenosis with a diameter reduction of respectively 70 and $80 \%$ in the proximal part of the left subclavian artery. Finally, ultrasonic duplex scanning found a permanent reversed direction of flow in the right vertebral artery in two patients with an arteriographically normal right subclavian artery. In one of these patients contrast arteriography showed an occlusion and in the other a stenosis with a diameter reduction of $70 \%$ of the innominate artery.

To summarize, in $6.5 \%(8 / 123)$ of the patients included in the second study, ultrasonic duplex scanning showed a transient or permanent reversed direction of flow in one or the other vertebral artery. In six of these eight patients the reversal of flow could be confirmed by contrast arteriography. In the remaining two patients arteriography did not permit a decision about the direction of flow in the relevant vertebral artery. However, in both patients there was a significant stenosis of the ipsilateral subclavian artery.

In the 999 patients investigated between December 1982 and October 1984 who were not included in the second study ultrasonic duplex scanning showed a latent, transient or permanent reversed direction of flow in one or the other vertebral artery in 47 patients $(4.7 \%$ ). More detailed information about these subclavian and innominate steal syndromes is shown in table 9.4.7/2.

Table $9.4 .7 / 2$

The 47 subclavian and innominate steal syndromes found in the patients investigated between December 1982 and October 1984, but who were not included in the second study.

RIGHT SIDE

LEFT SIDE

subclavian steal syndrome

latent : 0

transient : 3

permanent : 8

tolal : 11 subclavian steal syndrome

$\begin{array}{ll}\text { latent } & : 2 \\ \text { transient } & : \mathbb{1 2} \\ \text { permantent } & : \mathbb{1 7} \\ \text { total } & : 31\end{array}$

In one patient there was an occlusion of the innominate artery plus an occlusion of the left subclavian artery with a permanent reversal of the direction of flow in both vertebral arteries. 
In about $70 \%$ of these patients the steal syndrome was confirmed by intravenous digital subtraction angiography. In the remaining $30 \%$, intravenous digital subtraction angiography revealed a renarkably slower flow or no perfusion at all of the relevant vertebral artery. In only one patient with an arteriographically proven subclavian steal syndrome on the left side a normal direction of flow was found by ultrasonic duplex scanning.

\subsubsection{DISCUSSION OF THE RESULTS OF THE SECOND STUDY}

\section{innominate artery}

During the second study the investigation of the innominate artery was routinely carried out. In two patients arteriography showed an abnormal origin of the right subclavian artery, i.e. directly from the aortic arch distal to the left subclavian artery. In these two patients, with a congenital absence of the innominate artery, we could not investigate the proximal part of the right subclavian artery with the duplex scanner and failed to find the innominate artery.

In 17 of the remaining 121 patients (14\%) the innominate artery was located too deeply and a non-invasive study of this vessel was impossible. However, in most patients it was possible to obtain a longitudinal image of the different parts of the innominate bifurcation and to perform a Doppler measurement in the distal part of the innominate artery and the proximal part of the right common carotid artery. As shown in figure $8.1 / 1$ the most proximal part of the right subclavian artery was usually discerned as well, but in spite of extensive manipulations with the scan head its curve remained perpendicular to the Doppler beam axis. Therefore, in about $30 \%$ of the patients it was not possible to obtain a sonagram at the site of the origin or the most proximal part of the right subclavian artery.

The tables 9.4.1/1 and 9.4.8/1 show that ultrasonic duplex scanning of the innominate artery is a very reliable test. For the detection of an obstructive lesion of $50 \%$ or more and occlusions it shows a sensitivity of $1(3 / 3)$ and a specificity of $0.99(79 / 80)$. In only one of the 80 innominate arteries with no obstructive lesions on arteriography or with a stenosis of less than $50 \%$ diameter reduction there was a false positive result. The negative predictive value and positive predictive value were respectively $\mathbb{1}(97 / 97)$ and $0.75(3 / 4)$. The former was of course biased by the high number of innominate arteries without significant disease.

In patients with a tight stenosis or occlusion of the innominate artery there is usually a steal from the right vertebral artery and sometimes from the right common carotid artery (innominate steal). Although it is not always possible to image the innominate artery and to measure the velocity of flow in this 
Table $9.4 .8 / 1$

Sensitivity, specificity, positive and negative predictive values, and the overall accuracy for identifying a stenosis of respectively $50 \%$ or more in the innominate, subclavian, and vertebral (ostium) arteries.

\begin{tabular}{|c|c|c|c|c|c|}
\hline VESSEL & sensitivity & specificity & $\begin{array}{c}\text { positive } \\
\text { predictive } \\
\text { value }\end{array}$ & $\begin{array}{c}\text { negative } \\
\text { predictive } \\
\text { value }\end{array}$ & $\begin{array}{c}\text { owerall } \\
\text { accuracy }\end{array}$ \\
\hline \multirow{5}{*}{$\begin{array}{l}\text { innominate } \\
\text { artery } \\
\text { right sub- } \\
\text { clavian artery } \\
\text { left sub- } \\
\text { clavian artery } \\
\text { vertebral } \\
\text { artery }\end{array}$} & 100 & 000 & 075 & 100 & 0.09 \\
\hline & 1.00 & 0.99 & 0.13 & 1.00 & 0.99 \\
\hline & 0.43 & 0.99 & 0.75 & 0.96 & 0.95 \\
\hline & 0.88 & 0.98 & 0.78 & 0.99 & 0.97 \\
\hline & 0.81 & 0.88 & 0.67 & 0.94 & 0.87 \\
\hline
\end{tabular}

vessel the combination of low and abnormal damped sonagrams in both the right subclavian and the right common carotid arteries is so typical of a significant stenosis of the innominate artery that it will seldom be missed.

In this study there was one patient with an occluded innominate artery. This occlusion was correctly identified with the duplex scanner by the combination of abnormal Doppler signals in the right subclavian and common carotid arteries and a complete steal from the right vertebral artery.

\section{subclavian artery}

In only one of the 123 patients investigated between December 1982 and October 1984 the results of the non-invasive study did not permit a decision upon the state of the origin of the right subclavian artery. In this patient it was noted that the sonagrams recorded in the right common carotid and subclavian arteries showed a post-stenotic warble and a significant stenosis of the innominate artery was suggested. In this case the arteriographic study showed a stenosis with a diameter reduction of $70 \%$ in the proximal part of the innominate artery.

Nevertheless, ultrasonic duplex scanning of the right subclavian artery 
appeared to be the most difficult part of the investigation of the vessels of the arch of the aorta. For the detection of an obstructive lesion of $50 \%$ or more it has a specificity, a positive predictive value and a negative predictive value of respectively $0.99(88 / 89), 0.75(3 / 4)$ and $0.96(88 / 92)$. There was a false positive result (table 9.4.2/1) in only one patient. At arteriography this artery showed an atherosclerotic plaque with a diameter reduction of $40 \%$.

However, the major problem was the relative high number of false negative results. Using the contrast arteriographic data as a standard, seven right subclavian arteries showed an obstructive lesion with a diameter reduction of $50 \%$ or more. Only three of these seven cases were correctly identified with the duplex scanner, resulting in a sensitivity of $0.43(3 / 7)$. In the remaining four patients the arteriographic study showed a stenosis with a diameter reduction of respectively $50,55,56$ and $60 \%$. In all these cases the most proximal part of the right subclavian artery was positioned perpendicular to the Doppler beam axis and a Doppler measurement could only be performed a few centimeters distal to its origin. Disturbances of the shape of the sonagrams recorded distal to a stenosis, return to normal further downstream and, in general, the less severe the stenosis the more rapid this transition takes place. In the case of an obstructive lesion of 50 to $60 \%$ diameter reduction, the disturbed sonagrams will return to normal approximately three centimeters downstream to the stenosis (Reneman et al., 1979). Therefore, these four moderate stenoses were underestimated with the duplex scanner during the second study. Nowadays, we only offer an opinion about the state of the proximal part of the right subclavian artery if it is possible to perform a Doppler measurement at the site of its origin from the innominate artery. In some patients it is even possible to obtain an image of the atherosclerotic plaques in the wall of the proximal segment of the right subclavian artery (fig. $8.1 .2 / 1)$. In this part of the study there was no patient with an occlusion of the right subclavian artery.

According to our experience it was seldom possible to image the aortic arch and the proximal parts of the left common carotid and subclavian arteries. During both studies it was rarely possible to measure the velocity of flow at the site of the origin of these arteries. Usually it was difficult to ascertain how proximally the most proximal Doppler measurement was performed.

In the case of the left subclavian artery the ostium of the vertebral artery can serve as an identification mark. As the site of the ostium of the left vertebral artery can vary considerably its localisation is only of limited help. Nevertheless, it is important to first investigate the left vertebral artery in order to find its origin from the subclavian artery and then if possible, to measure the velocity of flow in the subclavian artery proximal to the ostium of the vertebral artery. 
Whilst recognizing these difficulties it was suspected that ultrasonic duplex scanning of the left subclavian artery would be more inaccurate than that of the right side. However, the tables $9.4 .2 / 2$ and $9.4 .8 / 1$ show that it is a reliable test. For the detection of an obstructive lesion with a diameter reduction of $50 \%$ or more and occlusions the specificity and sensitivity were respectively $0.98(84 / 86)$ and $0.88(7 / 8)$. Both the negative predictive value $(0.99$, $84 / 85)$ and the positive predictive value $(0.78,7 / 9)$ were remarkably high. In 11 left subclavian arteries the arteriographic study showed a mild stenosis (1$49 \%$ diameter reduction) and in only two of these 11 cases the degree of stenosis was overestimated with the duplex scanner. In these two cases the degree of stenosis was arteriographically estimated to be respectively 20 and $40 \%$ diameter reduction. Using the contrast arteriographic data as a standard, six left subclavian arteries showed a marked stenosis (50-99\% diameter reduction). In only one of these six arteries the data of the non-invasive study was normal and the stenosis was missed. In this patient the arteriogram showed a stenosis with a diameter reduction of $60 \%$ at the site of the origin of the left subclavian artery from the aortic arch. Probably the sonagrams recorded in this artery were obtained too far downstream to this stenosis.

In this part of the study there were two patients with an occlusion of the left subclavian artery. In one of these patients there was a complete steal from the left vertebral artery and in the remaining patient the flow reversal in the left vertebral artery was cardiac cycle dependent (transient steal). In both cases abnormally low signals were found with the duplex scanner in the proximal part of the subclavian arteries, while the occlusions were correctly identified by the combination of these abnormal signals and flow reversal in the ipsilateral vertebral arteries.

\section{vertebral artery (prevertebral segment)}

During the period of the second study we compared the data of the arteriographic study of the ostium of the vertebral artery with the data of ultrasonic duplex scanning in 120 vessels. The tables $9.4 .3 / 1$ and $9.4 .8 / 1$ show that ultrasonic duplex scanning of the ostium of the vertebral artery is a reliable test. For the detection of an obstructive lesion with a diameter reduction of $50 \%$ or more at the site of the ostium, a sensitivity of $0.81(22 / 27)$ and a specificity of $0.88(82 / 93)$ was found. The predictive value of a normal test was high $0.94(82 / 87)$, whereas the predictive value of a positive test was 0.67 (22/33). Considering the contrast arteriographic data as a standard, 73 vertebral arteries showed a normal ostium and in 20 arteries there was a mild stenosis ( $1-49 \%$ diameter reduction) at the site of the ostium. In 11 of these 93 cases $(12 \%)$ the degree of stenosis was overestimated with the duplex scanner. In only two of these 11 cases the ostium was quite normal on arteriography. 
In the remaining nine arteries arteriography indicated a stenosis with a diameter reduction of respectively $10 \%$ (once), $20 \%$ (twice), $30 \%$ (once), $40 \%$ (three times), and $45 \%$ (twice).

In 27 cases the arteriographic study showed an obstructive lesion of $50 \%$ or more and 22 of these 27 marked stenoses were correctly identified with ultrasonic duplex scanning. In five of these 27 arteries $(19 \%)$ the degree of stenosis was underestimated. In these five false negative results arteriography showed a stenosis with a diameter reduction of respectively $50,55,66,70$, and $83 \%$.

As in the first study, one of the major problems of the investigation of the ostium of the vertebral artery was the relatively high number of false positive results. In this part of the study it was realized that a vertebral artery may serve as a major collateral channel that bypasses a significant obstruction of the contralateral vertebral artery or, depending on the state of the circle of Willis, a significant stenosis of one or both internal carotid arteries. Generally, an increase in velocity occurs in the major collateral channels that immediately bypass a haemodynamically significant obstruction. This increase in velocity is related to the pressure drop in the collateral channel. This pressure drop may be divided into five principle components: gravity, flow acceleration, viscous loss of Poiseuille, convective loss of Bernoulli, and boundary layer loss (Spencer et al., 1984). At the site of the ostium of a vertebral artery which serves as a major collateral channel, the situation will be complicated but in general it may be assumed that the pressure drop is proportional to the square of the velocity of blood in the vertebral artery. Figure 9.4.8/1 shows that the velocity profile in a vertebral artery which serves as a collateral channel of the cerebral circulation is flattened.

If a vertebral artery serves as a collateral channel of some magnitude it may become dilated after several months. The ostium of the vertebral artery, however, is a fibrotic ring in the wall of the subclavian artery that can not dilate. Therefore, if a vertebral artery serves as a collateral chanmel and dilates, for example, from two to four millimeters, this fibrotic ring will act as a functional stenosis with a diameter reduction of $50 \%$. Therefore, when interpreting disturbances of flow in a vertebral artery in patients with multi-level obstructive disease, one should bear in mind that disturbed flow may occur in a normal vertebral artery, especially at the site of the ostium, with a compensatory high-flow state.

The first patient in whom we noticed a compensatory function of a vertebral artery was a 61 year old male with a history of frequent attacks of amaurosis fugax of both eyes for several months. There were no other signs and symptoms of cerebrovascular insufficiency. Ultrasonic duplex scanning and contrast arteriography showed an occlusion of the right vertebral, right common carotid, and right internal carotid arteries. Moreover, there was a 


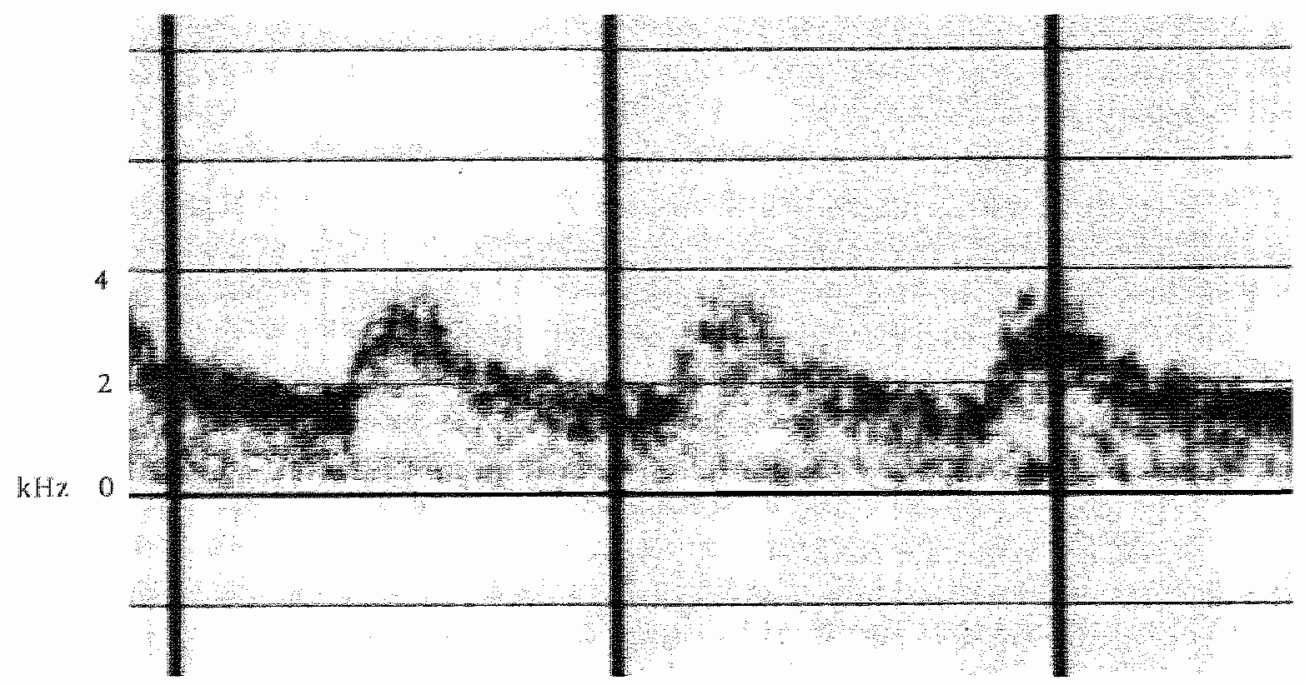

Figure $9.4 .8 / 1$

The Fourier transform of the Doppler signals recorded in a vertebral artery with a compensatory high-flow state.

subtotal stenosis of the left internal and external carotid arteries, and a stenosis of the innominate, right subclavian, and left subclavian arteries with a diameter reduction of respectively 33,40 , and $15 \%$. The right external carotid artery was perfused by collateral channels from the right thyrocervical trunk via the right ascending cervical and occipital arteries. Additionally, on both sides there was a reversal of the direction of flow in the peri-orbital arteries. Arteriographic examination of the intracranial vessels indicated a complete circle of Willis. If selective arteriography of the left vertebral artery was performed with manual compression of the left common carotid artery the former supplied all intracranial vessels with blood. This dilated vertebral artery had approximately the same caliber as the left common carotid artery. On arteriography it showed a smooth tapering stenosis with a diameter reduction of about $50 \%$ at the site of its origin from the left subclavian artery without significant signs of atherosclerosis (figure $9.4 .8 / 2$ ). Probably this stenosis was mainly caused by compensatory dilatation of the vertebral artery while the fibrotic ring of its origin in the wall of the subclavian artery maintained its original size. The sonagrams recorded in this vertebral artery are shown in figure 9.4.8/3. The relative high velocities during the entire cardiac cycle indicate a high-flow state in this vessel. After endarterectomy of the left carotid bifurcation the sonagrams recorded in the cervical segment of the left vertebral artery were more or less normalized. However, at the site of the ostium they still showed high and turbulent velocities indicative of a marked stenosis at this location. 


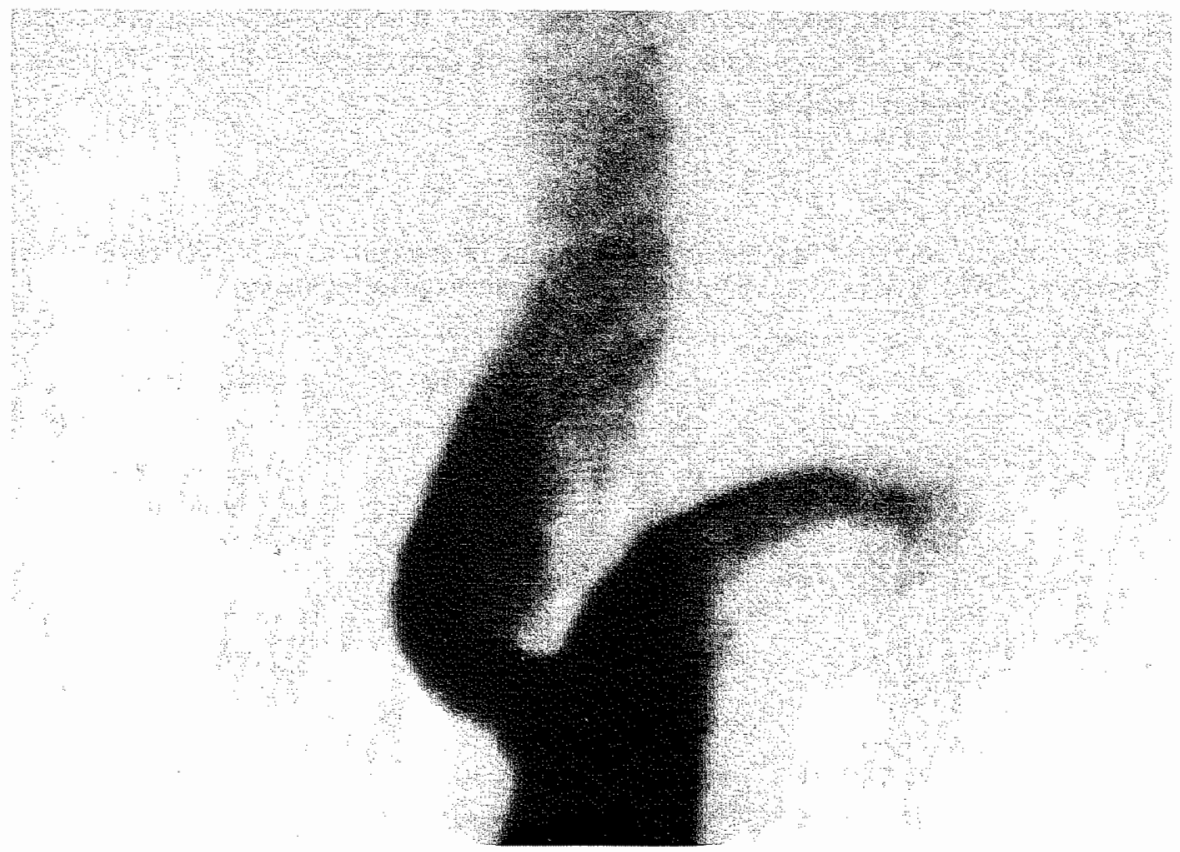

Figure 9.4.8/2

The dilated left vertebral artery with a compensatory function in a patient with severe multi-level disease. This vessel perfused almost the entire brain with blood and showed a relative stenosis without significant atherosclerosis at its origin.

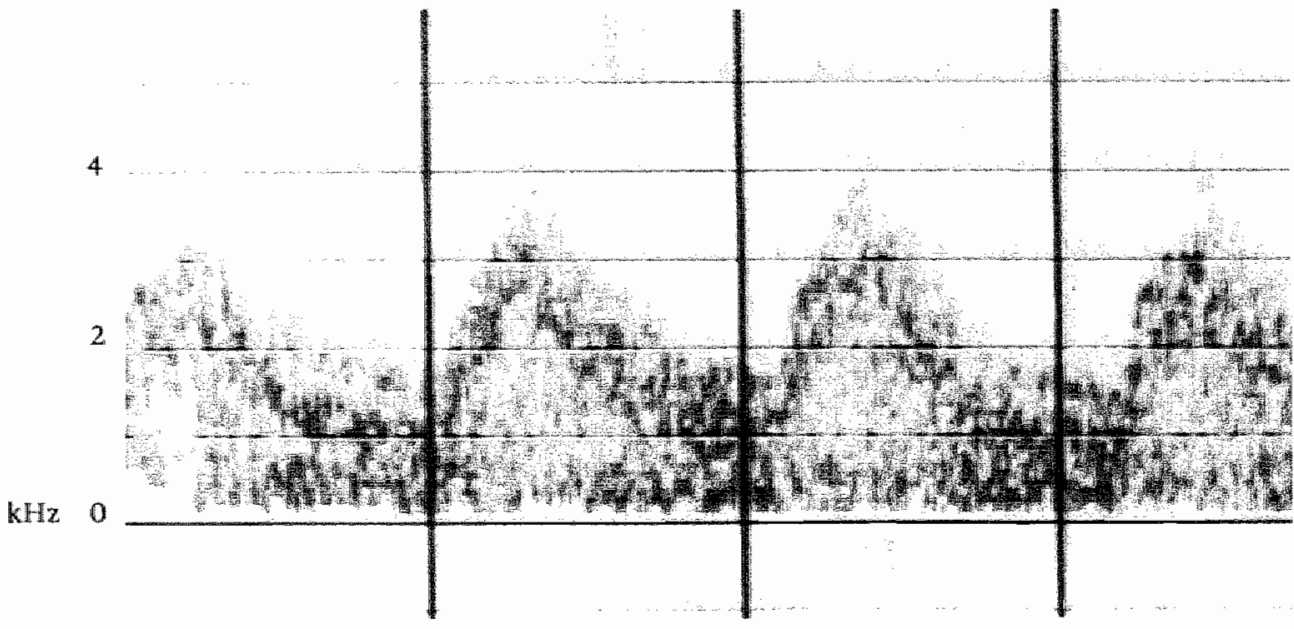

Figure $9.4 .8 / 3$

The Fourier transform of the Doppler signals recorded in the left vertebral attery of fig. 9.4.8/2. Note the relatively high velocities during the entire cardiac cycle. 
A second example was a 52 year old male with a history of amaurosis fugax. of the right eye and a reversible, intermittent neurological deficit of the left cerebral hemisphere (table $9.4 .8 / 2$, case 6 ).

Table 9.4.8/2.

The 11 cases in whom ultrasonic duplex scanning of the ostium of the vertebral artery gave a false positive result as compared with contrast arteriography.

CASE

ARTERY

STENOSIS

COMMENTS

1.

R. VERT.

L. vert.

R. comm. car.

L. comm. car.
0

99

50

70
Takayasu's disease, no follow-up studies.

2.

R. VERT.

L. vert.

R. int. car.

L. comm. car.

0
$>$
90
90

No follow-up studies.

3.

$\begin{array}{lr}\text { R. VERT. } & \mathbf{1 0} \\ \text { L. vert. } & 100 \\ \text { R. int. car. } & 50 \\ \text { L. int. car. } & 20\end{array}$

4.

R. VERT.

L. vert.

R. int. car.

L. int. car.
10

100

20

\section{0}

No follow-up studies.
20 hypoplastic 100 50-65

5.

R. vert.

L. VERT.

$R$. int. car.

L. int. car.
0

20

90-99

90-99
The next duplex study showed a high-flow state in the right vertebral artery and again high and turbulent Doppler signals at the site of its ostium.
Treatment: endarterectomy of both internal carotid arteries. After surgery the duplex study again showed high and turbulent Doppler signals at the site of the ostium of the left vertebral artery. 
6.
R. vert.
80
L. VERT.
R. int. car.
L. int. car.
90-99

Treatment: endarterectomy of the left internal carotid artery. After surgery the duplex study again showed high and turbulent Doppler signals at the site of the ostium of the left vertebral artery.

7.
R. vert.
L. VERT.
R. int. car.
L. int. car.

100

40

50-65

100

Treatment: extraintracranial bypass surgery on the left side. After surgery the results of the duplex examination at the site of the ostium of the left vertebral artery did not change.

8.

R. VERT.

L. vert.

R. int. car.

L. int. car.

9.

\section{R. VERT.}

L. vert.

$R$. int. car.

L. int. car.
40

100

100

$50-65$
No follow-up studies.
40

100

20

$90-99$
Treatment: endarterectomy of the left internal carotid artery.

After surgery the Doppler signals recorded in the right vertebral artery were normalized.

10.

R. vert.

L. VERT.

$R$. int. car.

L. int . car.
No follow-up

studies. 
11.
R. VERT .
L. vert.
$R$. int. car.
L. int. car.

Treatment: end-

arterectomy of the

right internal

carotid artery.

After surgery the results of the duplex examination at the site of the ostium of the right vertebral artery did not change.

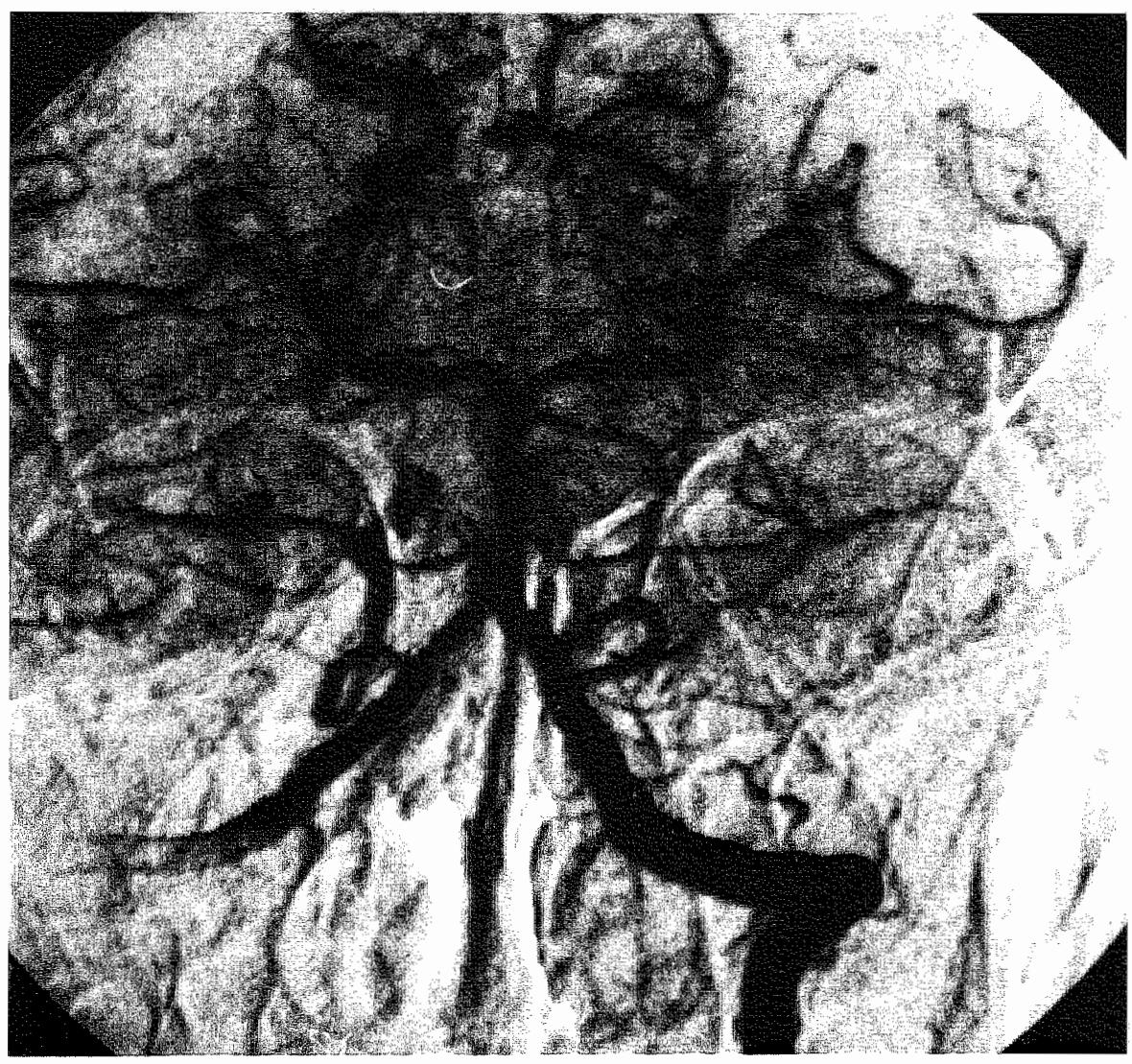

Figure 9.4.8/4

A semiselective arteriogram of the left vertebral artery of case 6 of table $9.4 .8 / 2$. Intracranially this artery perfused all vessels of the posterior fossa and both posterior cerebral arteries. The right vertebral artery of this patient showed a pressure reducing stenosis with a diameter reduction of $80 \%$ at its origin. 
This patient was referred to our hospital for a carotid endarterectomy. Biplane carotid arteriography was performed in another hospital and showed a subtotal stenosis of the left internal carotid artery and a stenosis of the right internal carotid artery with a diameter reduction of $50 \%$. Apart from these stenoses ultrasonic duplex scanning of the vertebral arteries showed very high and turbulent Doppler signals at the site of the ostia of both vertebral arteries. For this reason, supplementary semiselective contrast arteriography of both vertebral arteries was performed in our hospital. This arteriographic study showed a complete separation of the anterior and posterior cerebral circulations and a stenosis with a diameter reduction of $80 \%$ at the site of the ostium of the right vertebral artery. However, on the left side in spite of many oblique views the maximum degree of stenosis amounted to a diameter reduction of nearly $30 \%$. This dominant left vertebral artery (RV/LV $=2 / 3$ ) intracranially perfused all vessels of the posterior fossa as well as both posterior cerebral arteries (fig. 9.4.8/4). The relatively large territory of this left vertebral artery was presumably the cause of the high and turbulent Doppler signals recorded at the site of its ostium. After a successful endarterectomy of the left internal carotid artery this patient was investigated several times with the duplex scanner. The Doppler spectra recorded at the site of the ostia of both vertebral arteries did not change as compared with the preoperative spectra.

After these two cases we realized that the effect of a compensatory highflow state of a vertebral artery on the Doppler signals recorded at the site of the ostium of this artery might be the explanation of the false positive results in at least some patients with multi-level obstructive disease. Table $9.4 .8 / 2$ presents more detailed information about the 11 patients in whom the duplex study overestimated the degree of stenosis at the site of the ostium of a vertebral artery. In all these patients at least three and often all four extracranial cerebral vessels were involved in the atherosclerotic process. Moreover, it is striking that in five of the six patients with at least one follow-up examination the results of ultrasonic duplex study of the ostium of the vertebral arteries did not change. It is unlikely that in these patients the false positive results of the non-invasive study were the consequence of a lack of carefulness of the investigators. In our opinion the explanation may be the extent to which the cerebral territory is supplied with blood by the relevant vertebral artery. Consequently, a complete intracranial arteriographic study is necessary in these patients. Therefore, it is a pity that in seven patients (case 1,2, $3,5,7,10$, and 11) the quality of the arteriographic study did not permit a decision upon the intracranial territory of the relevant vertebral artery. In the cases 4 and 8 the intracranial wascular anatomy was more or less the same as in the aforementioned case 6 . The arteriographic study showed a complete 
separation of the anterior and posterior circulations. In both patients one vertebral artery was occluded or hypoplastic, while the other vertebral artery supplied all vessels of the posterior fossa and both posterior cerebral arteries with blood. Moreover, in case 4 the sonagrams recorded in the cervical segment of the right vertebral artery showed high flow velocities. The most interesting patient was case 9 . This patient had an occlusion of the left vertebral artery and a subtotal stenosis of the left internal carotid artery. Selective arteriography of the right vertebral artery showed that this artery supplied all vessels of the posterior fossa, both posterior cerebral arteries, and partly the left middle cerebral artery with blood. The sonagrams recorded in the cervical segment of the right vertebral artery showed a high-flow state. After endarterectomy of the left internal carotid artery the sonagrams recorded at the site of the ostium or more distally in the right vertebral artery were normalized. Obviously the intracranial territory of the right vertebral artery was now partly supplied by the left internal carotid artery.

The next important problem was the fact that in about $20 \%$ of the cases in whom arteriography showed a stenosis of $50 \%$ or more at the site of the ostium of a vertebral artery, this obstruction was not detected with the duplex scanner. Table $9.4 .8 / 3$ gives more detailed information about the five patients in whom the duplex examination did not diagnose a stenosis with a diameter reduction of $50 \%$ or more at the site of the ostium of a vertebral artery. It is striking that all these five patients have multi-level obstructive disease.

Table $9.4 .8 / 3$

The five cases in whom ultrasonic duplex scanning of the ostium of the vertebral artery gave a false negative result as compared with contrast arteriography.

CASE ARTERY STENOSIS COMMENTS *

1.

\section{R. VERT.}

L. vert.

$R$. int. car

L. comm. car.
50

$65-90$

100
Several follow-up duplex studies again showed mormal Doppler signals

at the site of the ostium of the right vertebral artery 
2.

R. VERT.

L. vert.

$\mathbb{R}$. int. car.

L. int. car.
55

57

$>65$

20
55

66

$90-99$

$65-90$
R. vert.

L. VERT.

R. int. car.

L. int. car.
0

70

90-99

50-65 (a)
$\mathbb{R}$, vert.

L. VERT.

$R$. int. car.

L. int. car.

$90-99$
$50-65$

Treatment: endarterectomy of the right internal carotid artery. After surgery the duplex study again showed normal Doppler signals at the site of the ostium of the right vertebral artery.
No surgical treatment. A follow-up duplex examination showed high and turbulent Doppler signals at the site of the ostium of the left vertebral artery.

4.

Arteriography showed an occlusion of the left vertebral artery proximal to the PICA. The duplex examination showed blunted signals in this vertebral artery, without forward velocities during diastole. No noninvasive follow-up studies.

5.

$\begin{array}{lc}\text { R. VERT. } & \mathbf{8 3} \\ \text { L. vert. } & - \\ \text { R. int. car. } & 20 \\ \text { L. int. car. } & 65\end{array}$

83

20

65
RV $/ \mathrm{LV}=3 / 5 \mathrm{Right}$ vertebral artery was a hypoplastic vessel with low velocities during the entire cardiac cyclie. A follow-up study again showed low Doppler signals at the site of the ostium.


In two patients (cases 1 and 2) the arteriographic study showed a stenosis with a maximum diameter reduction of respectively 50 and $55 \%$. In these two cases the disagreement between arteriography and ultrasonic duplex scanning was very small and could be explained by the limitations of measuring the degree of stenosis on the angiograms or by the inaccuracy of measuring the angle of insonation of the Doppler beam relative to the vessel axis, or both. In case 3 a follow-up examination study did show high and turbulent Doppler signals at the site of the ostium of the left vertebral artery. In this case the most proximal part of the left wertebral artery was probably not well discerned during the first study and the Doppler measurements were performed, therefore, too distally to its origin. Case 4 was a patient with complicated multi-level disease. The arteriographic study showed a stenosis with a diameter reduction of $70 \%$ at the site of the ostium of the left wertebral artery. This vertebral artery was occluded intracranially just proximal to the origin of the left posterior inferior cerebellar artery. In the right vertebral artery, which was normal at its origin, there was a stenosis with a diameter reduction of 70$80 \%$ in the intradural segment. The Doppler signals recorded at the ostium and in the cervical segment of the right vertebral artery were normal. However, in the left vertebral artery, which was of a relatively small caliber, the Doppler signals were blunted with very low systolic velocities and without forward velocities during diastole (fig. 9.4.8/5). This kind of Doppler signal is typical of an artery, supplying a vascular bed with a relatively high peripheral resistance. At the moment we know that a normal vertebral artery, even in the case of a relatively small caliber, always has forward velocities during the entire cardiac cycle. Although this phenomenon was recognized during the duplex examination of this patient, we did not realize that it was impossible to come to a decision about a more proximally located obstruction in an artery with a very low-flow state. In case 5 this problem was again anticipated. In this patient the right vertebral artery, which on arteriography showed a marked stenosis at its origin, was a hypoplastic vessel with low velocities during the entire cardiac cycle. In retrospect, it was incorrect to give an interpretation about the state of the ostium of this vertebral artery.

It is important to report that all five false negative results, as discussed here in more detail, occurred during the first seven months of the second study. Later on, with more experienced technicians and being aware of the possible causes of false negative results, they not longer occurred during the course of the second study.

Between December 1982 and October 1984 we failed to find a vertebral artery with the duplex scanner in 24 instances. Compared with the results of the first study the matter is now more complicated and therefore needs more 


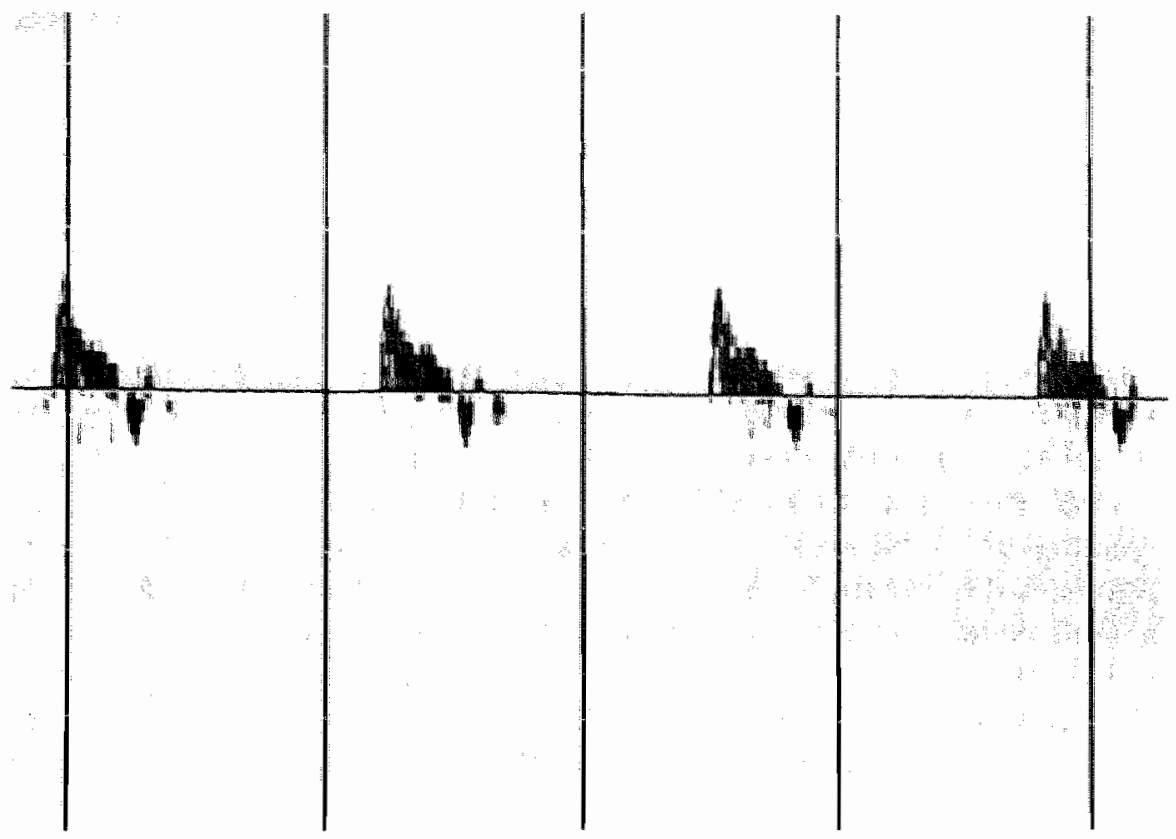

Figure 9.4.8/5

The Fourier transform of the blunted Doppler signals recorded in an intracranially occluded vertebral artery.

detailed discussion. As shown in table $9.4 .3 / 2$ in 20 of these 24 instances $(83 \%)$ the arteriographic study showed an occlusion of the vertebral artery at its origin from the subclavian artery. In three of these 20 occlusions we could not image the vertebral artery either in its prevertebral or in its cervical seqmint. In these three cases the situation was the same as in the first study. However, in seven cases we could image a vertebral artery, mostly in its cervical segment, but a Doppler measurement was impracticable. In these seven cases the report of the duplex study was: probably an occlusion of the vertebral artery. In the remaining ten cases we could not image the vertebral artery in its prevertebral segment. However, the cervical segment of the veritebrat artery was well discerned, usually with an antegrade direction of flow. In some of these cases the velocities as measured in the cervical segment signifycantle increased from the proximal to the distal part. In these ten cases the conclusion of the duplex study was: a proximally occluded vertebral artery which is probably perfused by collateral channels more distally. In nine of these ten cases the arteriographic study confirmed these collateral channels, usually from the ipsilateral thyrocervical trunk via the ascending cervical artery. In the remaining case contrast arteriography did not permit a decision about such collateral channels. It is interesting to point out here that in this

129 
wertebral artery the duplex examination showed a cardiac cycle dependent velocity direction.

As shown in table $9.4 .3 / 2$ the arteriographic study showed a normal vertebral artery in two cases. In both cases this vertebral artery was significantly smaller than its counterpart and in one of these two cases the results of the duplex examination were influenced in a negative way by the obesity of the patient. In the remaining two cases the arteriographic study showed a stenosis with a diameter reduction of $50 \%$ or more at the site of the ostium. In both instances the vertebral artery was a hypoplastic vessel and in one of these two vessels it showed a subtotal stenosis at its origin and a very low flow during the arteriographic examination.

According to these results, in the event of an occlusion, ultrasonic duplex scanning of the vertebral arteries is much more accurate, compared with the results of the first study, and in many cases it provides detailed information about collateral filling of the vertebral artery in its cervical segment.

As mentioned in section 9.4.3, during the second period of the study, the results of ultrasonic duplex scanning did not permit a decision concerning the state of the ostium of the vertebral artery in 65 cases of a total of 245 arteries $(27 \%)$. In 11 of these 65 cases the vertebral artery was well discerned along its whole length and a Doppler measurement at the site of the ostium was possible, but an abnormal high or low-flow state of the vertebral artery did not permit a decision upon the state of its origin. However, in the remaining 54 arteries the ostium was too deeply located, the most proximal part of the prevertebral segment was usually not well discerned and a Doppler measurement at the site of the ostium was impracticable. This occurred on the right side in 12 instances ( $22 \%$ ) and on the left side in 42 instances $(78 \%)$.

In conclusion, only depending on local anatomical properties, ultrasonic duplex scanning of the vertebral artery failed to make a decision about the state of its origin from the subclavian artery in $220 \%(54 / 245)$ of the patients. Sometimes the tortuous course of the prevertebral segment of an atherosclerotic changed vertebral artery misled the technician. The vertebral artery was well discerned just proximal to the transverse process of the sixth cervical vertebra, but it was impossible to follow the artery in a proximal direction as far as its ostium. However, in most of these 54 patients the ostium was too deeply located; the most proximal part of the vertebral artery was not well imaged and a Doppler measurement was impracticable. As a result of differences in the anatomical location of the ostia, this happened in more than three fourths of the patients on the left side. A better adjustment of the range of the scan head is very important especially in the case of this usually deeper located ostium. 
As described in section 9.4 .3 the number of patients with an atherosclerotic lesion in the remaining part of the prevertebral segment of the vertebral artery is very small. Therefore, at the moment a discussion of the data of ultrasonic duplex scanning of this segment of the vertebral artery, as compared with contrast arteriography is not indicated.

\section{vertebral artery (cervical segment)}

In $79 \%$ (97/123) of the patients included in the second study a comparison of the results as obtained with ultrasonic duplex scanning and those obtained with contrast arteriography for the cervical segment of the vertebral artery was possible. There was a striking agreement between the data of both investigations. In five patients $(5 \%$, case $2,3,4,6$, and 7$)$ we could demonstrate a displacement of the vertebral artery in its cervical segment by osteo-arthritic spurs with both the duplex scanner and contrast arteriography. In two of these five patients the displacement of the vertebral artery only appeared on the right side and in three patients bilaterally. In none of these five patients there was any sign of an atherosclerotic lesion in the cervical segment of the vertebral artery.

In four other patients (case 1, 5, 8, and 9) high and turbulent Doppler signals pointed to a stenosis with a diameter reduction of $50 \%$ or more in the cervical segment of the vertebral artery without displacement of the artery by osteo-arthritic spurs of the cervical vertebrae. Using contrast arteriography as a standard, in two of these four patients (case 8 and 9) the degree of stenosis was overestimated with the duplex scanner. In both patients the sonagrams recorded in these vertebral arteries showed high flow velocities. As explained in section 9.4.3, these high flow velocities are probably an important reason for the overestimation of a mild to moderate stenosis (diameter reduction less than $50 \%$ ) in the vertebral artery. In the remaining two patients arteriography revealed a stenosis with a diameter reduction of 50-70\% at C6-C5 (case 1) and of $60 \%$ at $\mathrm{C} 4$ (case 5), respectively. In these two patients there was a complete agreement between the data of ultrasonic duplex scanning and contrast arteriography.

In the last patient (case 10) ultrasonic duplex scanning showed a displacement of the vertebral artery in its cervical segment with normal Doppler signals at this location. Contrast arteriography of this vertebral artery was only available in one direction and showed a stenosis with a diameter reduction of $30-40 \%$. Considering our criteria as discussed in section 8.2 , the sonagrams recorded at the side of a moderate stenosis should be normal in a vertebral artery without high flow velocities. This is the only patient in our material with a combination of a displacement of the vertebral artery by osteo-arthritic spurs and an atherosclerotic obstructive lesion at the same 
location. Stein and co-workers (1962) also described no increased atherosclerosis of the vertebral artery in patients with cervical spondylosis.

During ultrasonic duplex scanning the exact location of a significant atherosclerotic lesion or displacement of the vertebral artery in its cervical segment by osteo-arthritic spurs is usually not a problem. While making a careful investigation of the vertebral artery in a cranio-caudal direction it is easy to count the number of transwerse processes of the cervical vertebrae in order to recognize the transverse process of the axis and to localize any abnormality in the cervical segment of the vertebral artery.

To summarize, six patients $(6 \%)$ showed displacement of at least one vertebral artery in its cervical segment by osteo-arthritic spurs of the cervical vertebrae. In fiwe of these six patients there were no indications for an atherosclerotic obstructive lesion in the cervical segment of the relevant vertebral arteries. In only one patient contrast arteriography showed a moderate stenosis of the right vertebral artery at the same level where the non-invasive examination revealed a displacement of the vertebral artery by cervical spondylosis. In the remaining four patients $(4 \%)$ an atherosclerotic lesion was located in the cervical segment of the vertebral artery without osteo-arthritic degenerative change of the cervical column. For completeness sake it must be emphasized here that in most patients contrast arteriography of the vertebral arteries was only performed with the head in the mid position. As discussed in section 5.3 compression of the vertebral artery by osteo-arthritic spurs is more clearly demonstrated on arteriography during rotation of the head. Ultrasonic duplex scanning of the vertebral artery can only be executed with the head turned to the contralateral side.

\section{vertebral artery (atlantic segment)}

The most distal part of the cervical segment and the atlantic segment of the vertebral artery are closely related to the occipito-atlanto-axial complex of the cervical column. This complex is one of the most complicated series of articulations of the human body. It serves as a transition zone between the standard vertebral joint structures and the radically different skull. The joint complex functions to support the head and it protects the spinal cord and adjacent vital structures while allowing the necessary ranges of motion. During movement of the head the occipito-atlanto-axial articulations are involved in rotation, flexion, extension, lateral bending and translatory movements. Although a recent review of the literature (Jofe et al., 1983) still indicates a considerable controversy concerning some aspects of the basic biomechanical and kinematic characteristics of the occipito-atlanto-axial complex, most investigators conclude that significant rotation only occurs at the atlanto-axial joint. The anatomical design of the occipito-atlanto joint prevents axial rota- 
tion. The joint's surfaces of the axis are cup shaped in the saggital plane with the arcuated occipital articular knobs fitting into it. On the other hand, there is extensive axial rotation at the atlanto-axial joint. This fact can also be explained by the geometry of the articular surfaces. They are convex with a horizontal orientation, a design that allows maximum mobility. About $50 \%$ of the axial rotation of the head takes place at the atlanto-axial joint. After 30 degrees of rotation of the head there is kinking of the contralateral vertebral artery. This kinking, which is also accompanied by stretching of the artery, occurs as the vertebral artery runs through the transverse foramen of the first and second cervical vertebrae. It becomes more marked as the angle of rotation is increased. At 45 degrees of rotation the ipsilateral artery begins similarly to kink (Fielding, 1957, Selecki, 1969). Occlusion of one vertebral artery does not itself necessarily reduce blood flow to the posterior fossa. However, if flow in both vertebral arteries is compromised symptoms related to vertebrobasilar ischaemia may be elicited. Rotational compression of the vertebral artery at the level of the atlanto-axial joint is described by many investigators in postmortem material (de Kleyn et al., 1933, Tatlow et al., 1957, Brown et al., 1963) as well as in vivo by contrast arteriography (Bauer et al., 1961, Ouchi et al., 1973, Barton et al., 1975). Bauer and colleagues (1961) noted rotational compression of the vertebral artery in so many patients that they considered this phenomenon as a normal variant.

Although ultrasonic duplex scanning of the most distal part of the cervical segment of the vertebral artery between the transwerse processes of the axis and atlas was routinely performed by us in every patient in whom the cervical segment was investigated, we made no definitive conclusion about the status of this part of the vertebral artery. In most patients the Doppler signals recorded in this segment of the vertebral artery were normal. In some patients, however, the sonagrams recorded in the kink between the axis and atlas were abnormal, but the acute angle of insonation made a reliable decision impossible. In other patients with severe multi-level atherosclerotic disease of the cerebral arteries, abnormal sonagrams recorded in the most distal part of the cervical segment of the vertebral arteries returned to normal after surgical treatment. Therefore, according to our experience the finding of abnormal high and turbulent Doppler signals in the vertebral artery at the level of the atlanto-axial articulation is a complicated matter that needs further investigation.

Atherosclerotic lesions in the atlantic segment of the vertebral artery are rare (section 4.2). In a thorough study with continuous wave Doppler sonagraphy on 4,468 patients Ringelstein (1984) demonstrated a significant stenosis in this segment of the vertebral artery in only seven patients. In six of these seven patients the obstructive lesion was confirmed by contrast arteriography. In the remaining patient the results of the Doppler study were considered as false positive. There were no false negative results. 
Contrast arteriography did not reveal a stenosis in the atlantic segment of the vertebral arteries in any of the patients included in this study.

\section{vertebral artery (intradural segment)}

During ultrasonic duplex scanning of the carotid arteries an occlusion of the internal carotid artery often can be confirmed by the blunted signals recorded in the ipsilateral common carotid artery. Flow in the common carotid artery than moves to zero during late systole or diastole and even to reverse in some instances. This phenomenon was described by Bodily and coworkers (1981) and Breslau and co-workers (1982). We also have used it successfully for a more reliable differentiation between a high grade stenosis and a total occlusion of the internal carotid artery. The same phenomenon can be proximally demonstrated in an internal carotid artery which is occluded more distally for example, at the level of the syphon. Ringelstein and associates (1983) used this 'Widerstandsprofil' as an important phenomenon in the diagnosis of intracranially located occlusions of the vertebral artery. However, it must be stressed that whenever flow to zero proximally in an artery is used to characterize a lesion more distally in that artery, the angle between the ultrasonic beam and the axis of the vessel should be optimal. Moreover, flow to zero in diastole with reversal of flow during late systole can be observed in the extracranial cerebral arteries of patients with aortic valve disease. In these cases, it is expected to be found bilaterally.

The results of the second study show that the phenomenon of blunted Doppler signals recorded proximally in a vertebral artery has a high positive predictive value in the assessment of a more distally located occlusion. Usually, these occlusions are located in the intradural segment of the vertebral artery. We never found blunted Doppler signals in a vertebral artery with a tight stenosis at the site of its origin. In these cases the Doppler signals were damped, with low flow velocities during the entire cardiac cycle. On the other hand, in four cases with an occlusion demonstrated at arteriography in the intradural segment of the vertebral artery, low flow velocities were recorded in these arteries, but the sonagrams did not confirm the distally located occlusion. The same kind of sonagrams can also be recorded in a relatively small vertebral artery with probably a small cerebral territory perfused by this artery (Table $9.4 .6 / 1$, case 6 ). In our opinion, the morphology of the sonagrams recorded in an intracranially occlucled vertebral artery will depend on the cerebral territory supplied with blood by this artery. For example, if the occlusion is located proximally to the origin of the posterior inferior cerebellar artery, this artery supplies a vascular bed with high resistance and flow will move to zero during diastole. However, if the occlusion is located distal to the origin of a posterior inferior cerebellar artery which perfuses a rela- 
tively large territory the peripheral resistance will be lower. Flow is then quasi-steady and just above zero during the entire cardiac cycle. "Thus, the negative predictive value of blunted Doppler signals in the assessment of an intracranially located occlusion of the vertebral artery is rather low. Moreover, the evaluation of atherosclerotic lesions in the intradural segment of a vertebral artery by ultrasonic duplex scanning is impossible. In all cases with an intracranially located stenosis with a diameter reduction of $50 \%$ or more the Doppler signals recorded more proximally in these arteries were normal.

In conclusion, ultrasonic duplex scanning of the vertebral artery has only a limited profit in the assessment of intracranially located atherosclerotic obstructive lesions. The morphology of the sonagrams recorded proximally in a vertebral artery will principally depend on the cerebral territory perfused by that artery. This can vary enormously from one case to another. In ultrasonic duplex scanning the caliber of the vertebral artery can provide useful information for a more reliable interpretation of the morphology of the sonagrams. However, even a relatively small and intracranially occluded vertebral artery may perfuse a posterior inferior cerebellar artery with a relatively large territory. Flow in this vertebral artery will then be low but just above zero during the entire cardiac cycle.

\section{subclavian and innominate steal syndromes}

In $6.5 \%$ of the patients included in the second study a subclavian or innominate steal syndrome was found. The percentage was slightly lower (4.7) for the group of patients also investigated between December 1982 and October 1984, but not included in the second study. An average number of about $5 \%$ of subclavian and innominate steal syndromes is twice as high as a percentage of 2.6 found by Fields and co-workers (1972) using arteriographic criteria. However, we also included patients with a latent or transient reversed direction of flow. These cases were usually more difficult to confirm by routine arteriography. In these cases the net direction of flow was forward but the perfusion of the relevant vertebral artery was much decreased and filling of the artery by contrast material was significantly delayed. In some patients there was a to and fro direction of flow in the vertebral artery with net no flow in any direction. In these cases the steal syndrome could only be demonstrated arteriographically by a careful investigation.

In patients with a pressure reducing stenosis of the subclavian or innominate artery the direction of net flow will depend on the demand for blood of both the limbs and cerebral vascular beds. As the balance between these demands for blood may vary from one moment to another, the net direction of blood flow in the vertebral artery may change from one investigation to another. In five of our patients with a subclavian steal syndrome the stage of 
subclavian steal changed from a transient to a permanent steal and vice versa during several follow-up studies. In one of these patients the direction of flow in the vertebral artery was sometimes normal. These findings showed that a subclavian or innominate steal syndrome was a dynamic pathophysiologic condition and the volume of reversed flow could very significantly. 


\subsection{INTRODUCTION}

Non-invasive techniques used in the evaluation of atherosclerotic disease have two main goals: (1) to detect and assess atherosclerosis in symptomatic patients and (2) to evaluate the progress of disease. Most of the techniques currently used in clinical medicine are included in a battery of tests. They are normally used as a screening test to select those patients who should undergo selective contrast arteriography, who should go to corrective surgery, or who could safely forego both contrast arteriography and surgery. For this purpose, in many instances a simple positive or negative test result is all that is required. Ideally, however, such a non-invasive technique should be able to identify both normal vessels and those with the entire spectrum of disease involvement with an acceptable accuracy. Since atherosclerosis is a disease which has a long latent period during which asymptomatic lesions grow slowly, such an ideal technique may give more insight in the natural history and may improve treatment. Therefore, a major future role for some noninvasive procedures may be the application in the detection and assesment of atherosclerosis in its presymptomatic phase by measuring rates of growth or change. When such a technique is used for this purpose the demands for precision and accuracy will increase for a procedure used to track lesion size over time must be able to detect the spontaneous growth rate of lesions and that has been shown to be up to $4 \%$ per year in the femoral arteries (Blankenhorn et al., 1978).

Nowadays two imaging procedures which offer such potential for preventive treatment of atherosclerosis are ultrasound and intravenous digital subtraction angiography. Both seem destined to have a major impact on the future practice of neurologists, neurosurgeons, vascular surgeons, and cardiologists. These new diagnostic procedures both for central and peripheral circulation, are leading to a collection of new physiological and anatonical data, more precise quantitative clinical observation, population screening, and of particular interest to this thesis, new extrapolation of vascular phenomena towards the understanding of disorders in the cerebral vasculature.

The aforementioned apply to many vascular beds of the human body, i.e. the aorta, the renal arteries, the limb arteries, the coronary arteries, and the extra- and intracranial cerebral arteries. Here we will restrict the discussion to the extracranial cerebral arteries, and especially to ultrasonic duplex scanning of the carotid and innominate-subclavian-vertebral arterial systems. 


\subsection{RECENT DEVELOPMENTS IN THE DIAGNOSIS OF ATHEROSCLEROTIC DISEASE IN THE CEREBROVASCULAR TREE}

In order to evaluate atherosclerotic disease of the cerebrovascular tree, three techniques have been introduced and tested clinically in the St. Antonius Hospital, Nieuwegein, The Netherlands during the last ten years: ocular pneumoplethysmography, intravenous digital subtraction angiography and ultrasonic duplex scanning. These three diagnostic procedures are now used in our hospital as screening tests in patients suspected of extra- and intracranial atherosclerotic disease. For a better understanding of the interaction of these tests and the possibilities and limitations they will each be discussed here briefly. As a matter of routine by means of these diagnostic tests we attempt to be informed about the status of the carotid and innominate-subclavianvertebral arterial systems extracranially and intracranially in all patients.

The assessment of atherosclerosis of the carotid system and its potential collateral circulation by ocular preumoplethysmography (OPG Gee) has been described by Eikelboom (1981). Ocular pneumoplethysmography is a simple and accurate test to trace a pressure reducing stenosis in the carotid arterial system from the level of the aorta to the intracranial internal carotid artery. The transition zone from non-pressure reducing to pressure reducing or haemodynamically significant stenosis appeared to be from $60 \%$ to $70 \%$ transverse diameter reduction. For the sake of convencience, stenosis with a diameter reduction of $65 \%$ or more and occlusions are considered as haemodynamically significant. However, it is not possible to determine at which level the obstructive lesion is located nor is it possible to predict the severity of the lesion by ocular pneumoplethysmography. The most important application when determining a patient's collateral circulation will be its possible use in recognizing the stroke prone patient. Routinely, the collateral circulation is tested by manual compression of the carotid arteries during ocular pneumoplethysmography as well as electroencephalography. The clinical significance of these diagnostic tests can only be evaluated in a prospective study, because the collateral circulation in stroke patients with occluded carotid arteries may be unreliable due to possible improvement of the collateral circulation after the occlusion (Liebman et al., 1981). However, in a prospective study Moll (1984) showed that the collateral ophthalmic artery pressure did not change significantly when a pressure reducing stenosis developed on the same side in the carotid artery.

The technique capable of imaging the small contrast levels produced after an intravenous injection of contrast material was first described by Mistretta and co-workers in 1973. Later the introduction of a digital video-image processor made this subtraction technique suitable for clinical use and in partic- 
ular for intravenous angiography of the cerebral arteries (Strother et al., 1980, Chilcote et al., 1981). Since then, the use of this technique has been met with great enthusiasm as an invasive technique with little hazard and morbidity for the delineation of atherosclerotic disease in many vascular areas. The accuracy and limitations of this technique in the evaluation of atherosclerotic cerebral vascular disease had been described by many investigators (e.g. Chilcote et al., 1981, Wood et al., 1983, Earnest et al., 1983, Folcarelli et al., 1983, Hoffman et al., 1984). Avoidance of arterial catheterization and performance on an outpatient basis accounted for the widespread acceptance of intravenous digital subtraction angiography for the assessment of atherosclerotic disease of the carotid bifurcations. Some reports (Lusby et al., 1982, Folcarelli et al., 1983) seem to indicate that clinical decisions regarding operative or non-operative treatment in many patients with carotid artery disease can be made solely on the basis of the data of this technique. On the other hand it has some inherent limitations. The most important are: poor quality of the images by swallow artifacts or overall artifacts, incompleteness of information by overlying of the external carotid or vertebral arteries, and poor arterial contrast density secondary to poor cardiac function. Moreover, it is very important to visualize the vessel of interest adequately in more than on projection. Hoffman and colleagues (1984) reviewed the examination of a routine cerebrovascular study and showed that in $42 \%$ of their cases the images of the carotid bifurcation were diagnostically of insufficient quality, These difficulties may require multiple anteroposterior and oblique views in many patients. This can result in the administration of large volumes of contrast material which may compromise renal function especially in the elderly patient. Another important limitation of intravenous digital subtraction angiography is the lower spatial resolution compared with conventional arteriography. Although intracranial vascular examinations, such as a search for deviations or occlusions of the carotid syphon or the basilar artery, are readily applicable, the examinations are often of insufficient quality to detect intracranial vascular disease (Earnest et al., 1983).

Intravenous digital subtraction angiography was introduced in our hospital in October 1980. De Vries (1984) investigated the accuracy of this technique and of conventional contrast arteriography in the evaluation of atherosclerotic disease of the carotid bifurcation by means of intra- and interobserver agreement, expressed in Kappa values.

The concept of combining a pulsed-echo system and a pulsed Doppler system into a so-called duplex scanner was first described by Barber and coworkers (1974). Since its initial development the system has undergone continuous modifications to make it usuable in clinical practice. This sequence and its first results were described by Phillips and associates (1980), soon 
followed by the results of many other investigators (i.e. Blackshear et al., 1979, 1980, Fell et al., 1981, Breslau, 1982, Zbornikova et al., 1982, Langlois et al.. 1983).

We introduced this technique in our hospital in May 1981. In combination with real time spectral analysis of the Doppler signals, ultrasonic duplex scanning appeared to be a reliable diagnostic procedure for the detection of atherosclerosis of the internal and external carotid arteries (Ackerstaff et al., 1982). Its most striking failure was its poor results in classifying normal vessels and those with minor lesions. The duplex scanner overestimated the degree of disease in such cases. This problem can partly be solved by the use of transducers with a more appropriate focal arrangement and smaller sample volumes (Knox et al.. 1982-a, van Merode et al., 1983) or by the combination of a multigated pulsed Doppler and a high resolution B-scanner (Hennerici et al., 1984). High resolution real time ultrasonic imaging $(7.5$ or $10 \mathrm{MHz}$ transducer) of the carotid bifurcation can especially provide valuable anatomical information about plaque morphology and sometimes ulcerations can be detected (Cooperberg et al., 1979, Ringelstein et al., 1982, James et al., 1982, Katz et al., 1983, Reilly et al., 1984). Since the increased axial resolution obtained by using transducers with relative high nominal frequencies results in increased absorbtion of the sound waves and limited depth penetration, the investigation of deeper located vessels, such as the prevertebral segment of the vertebral artery and the branches of the aortic arch, is usually impracticable with these transducers. Moreover, because reflections from the vessel wall are poor if the angle of the ultrasound beam is not perpendicular, the examination of the often tortuous, smaller and more deeply located prevertebral segment of the vertebral artery can only be performed satisfactorily with a relative small transducer with a rounded surface. Such a transducer is more handsome and easier to manipulate. By using the standard mechanical sector scanners of our system it was relatively easy to find the vertebral artery just proximal to the transverse process of the sixth cervical vertebra, to follow the artery in its prevertebral segment from a distal to a proximal position and to find its origin from the subclavian artery. The investigation of the cervical segment of the vertebral artery, the most proximal part of the right common carotid artery, and the subclavian and innominate arteries was also possible with the same transducer in most of the patients. Our experiences with the recently developed $7.5 \mathrm{MHz}$ and the multifrequency $(3,5$, and $7.5 \mathrm{MHz}$ ) tranducers are encouraging. The investigation of the ostium and the most proximal part of the vertebral is especially practicable with these transducers. 

PATIENTS

It will be clear that the aforementioned techniques used by us in the evaluation of atherosclerotic disease of the cerebrovascular tree all have their individual possibilities and limitations. In our opinion, only the consistent use of them as a battery of screening tests makes it possible to be adequately and reliably informed about the status of the extra- and intracranial cerebral arteries in most of the patients without the use of intra-arterial contrast angiography. This invasive diagnostic procedure will only be performed by us in symptomatic patients if there is significant disagreement between the data of ocular pneumoplethysmography, carotid compression tests, intravenous digital subtraction angiography and ultrasonic duplex scanning. This is also the case if the clinical findings make more detailed information necessary about a part of the cerebrovascular tree with special interest, such as the ostia of the vertebral arteries or the intracranial arteries. This policy resulted in a reduction of the number of conventional contrast arteriograms performed on patients suspected of cerebrovascular atherosclerosis of about $80 \%$ during the first year and about $90 \%$ during the second year (de Vries, 1984). Previously, however, we compared the data of intravenous digital subtraction angiography and ultrasonic duplex scanning with those of conventional contrast arteriography with regard to their accuracy in quantifying internal carotid artery disease (Eikelboom et al., 1983). This study showed that with a combination of intravenous digital subtraction angiography and ultrasonic duplex scanning it was possible to identify both normal vessels and those with different degrees of disease involvement with acceptable accuracy as compared with contrast arteriography. Moreover, for the last two years we used the same parameter as described by Knox and co-workers (1982-b) to grade ob. structive lesions in the internal carotid artery with a diameter reduction of $50 \%$ or more in three subgroups, namely: $50-64 \%, 65-89 \%$ and $90-99 \%$ diameter reduction. Although our results seem to provide a useful method in clinical practice, further evaluation is necessary to compare these results with those of ocular pneumoplethysmography and conventional contrast arteriography. Since we started this study in 1983 results are now becoming available and will be soon published.

Cerebral atherosclerosis is a diffuse process which involves both the carotid and the innominate-subclavian-vertebral arterial systems in many symptomatic and asymptomatic patients. Usually, however, the physicians are not well informed of the distribution of atherosclerotic lesions over both systems. At the same time, it is well known that in patients with diffuse and severe 
atherosclerosis of the cerebral vessels the interdependence of the anterior and posterior circulations may be very important for the final outcome of the patient. In the first place, this interdependence will be defined by the circle of Willis. Secondly, in the event of a functionally well developed posterior communicating artery on one or both sides, this interdependence will depend on the degree of atherosclerosis of both the carotid and innominate-subclavianvertebral arterial systems intracranially and extracranially. In such cases with severe obstructive disease of the carotid arteries a vertebral artery may serve as a major collateral channel of the cerebral circulation. Moreover, in patients with a complete separation of the anterior and posterior circulations, especially pressure reducing stenoses of the innominate, subclavian or vertebral artery may cause transient or permanent neurological deficit in the vertebrobasilar territory. The diffuse distribution of cerebral atherosclerosis and the complexity of its pathology ensured our search for a safe and accurate diagnostic procedure which could play a potentially powerful role in the evaluation of atherosclerosis of the innominate, subclavian and vertebral arteries. We tried to evaluate atherosclerosis of these vessels by ultrasonic duplex scanning.

We started with ultrasonic duplex scanning of the carotid arteries in May 1981. After a learning phase of four months we also tried to investigate the subclavian arteries and the most proximal part of the vertebral arteries on both sides. The results of ultrasonic duplex scanning of these arteries as compared with contrast arteriography were so encouraging (Ackerstaff et al., 1984) that we decided to investigate on both sides the extracranial part of the cerebrovascular tree as completely as possible. During the period of the second study, from December 1982 to October 1984 , we routinely investigated the innominate, subclavian, internal and external carotid arteries and the prevertebral segment of the vertebral arteries. The investigation of the cervical segment of the vertebral artery was only performed if the referring physician requested it or if the patient had a history of vertebrobasilar insufficiency. The interaction between atherosclerotic lesions and displacement or obstruction of the vertebtal artery by cervical spondylosis was especially evaluated for this segment. The cervical segment of the vertebral artery was also routinely investigated between the transverse processes of the second and first cervical vertebrae. However, an obvious relation between abnormal sonagrams recorded in this part of the vertebral artery and rotation of the head was not found. In some patients with diffuse and severe atherosclerotic disease of the cerebral arteries abnormally high and turbulent Doppler signals were found in the vertebral artery at the level of the atlanto-axial articulation. Moreover, we evaluated the significance of ultrasonic duplex scanning of the vertebral artery in the event of a severe obstructive lesion in the intradural segment of this artery. Finally, for the sake of completeness we also noted the number of patients with a subclavian or innominate steal syndrome. 


\subsection{ULTRASONIC DUPLEX SCANNING OF THE INNOMINATE, SUBCLAVIAN, AND VERTEBRAL ARTERIES}

It was not possible to image the origin of the innominate artery from the aortic arch in any of the 1122 patients investigated during the second study. However, investigation of the distal part of this artery was usually no problem. In only $14 \%$ of the patients the investigation of the innominate artery was not possible at all. In most of these patients the proximal part of this artery was located too deeply. Only a few patients $(0.25 \%$, Daseler, 1959) will have an abnormal origin of the right subclavian artery directly from the aortic arch distal to the origin of the left subclavian artery. As the investigation of the innominate artery was only practicable in its most distal part, in general the Doppler signals were recorded 2-4 centimeters distal to its origin from the aortic arch. Usually, however, atherosclerotic lesions are located in the most proximal part of the innominate artery, very close to its origin. Disturbances of the shape of the sonagrams recorded distal to a stenosis return to normal further downstream. In general, the less severe the stenosis the more rapid this transition takes place. In the case of a stenosis with a diameter reduction of 50 to $60 \%$ at the site of the origin of the innominate artery the disturbed sonagrams will return to normal approximately three centimeters downstream (Reneman et al., 1979), in other words just in the distal part of the innominate artery. Therefore, atherosclerotic lesions of the innominate artery with a diameter reduction of less than $50 \%$ will usually not give disturbed signals in the distal part of this artery and will not be detected with ultrasonic duplex scanning.

Investigation of the subclavian arteries was practicable in almost all patients. In the first study, in which the data of the right and the left side were not separated, the results of ultrasonic duplex scanning were of insufficient quality in 7 of 163 subclavian arteries $(4 \%)$. During the second study the investigation was impracticable in only one patient $(0.9 \%)$ on the right side and in none of the patients on the left side. In this patient the results were unreliable because of a significant stenosis of the innominate artery. In such cases it may be difficult to distinguish the flow disturbances as induced by the lesion of the innominate artery from the disturbances induced by a possible second lesion at the site of the origin of the right subclavian artery. In spite of these few failures, ultrasonic duplex scanning of the right subclavian artery was the most difficult part of the investigation of the vessels of the arch of the aorta. The most proximal part of the right subclavian artery makes a posterolateral curve and, in spite of many efforts, the axis of the Doppler beam remained perpendicular to the axis of the vessel and a Doppler measurement in the most proximal part of this artery was impracticable in about $30 \%$ of the patients. However, in most of these patients the proximal part of the right 
subclavian artery was well imaged and in some patients an atherosclerotic lesion at the site of its origin was very well discerned.

In only a few patients was it possible to image the origin of the left subclavian artery and in almost all patients the Doppler signals were recorded roughly $1-4$ centimeters downstream to the origin of this artery from the aortic arch. Therefore, it is striking that the investigation of the left subclavian artery was more accurate than on the right side. According to our experience atherosclerotic lesions of the left subclavian artery were more diffuselly located in the proximal part of this artery than in the right subclavian artery. In many patients the lesions expanded towards the site of the ostium of the left vertebral artery. This was probably the reason for the relatively low number of false negative results in the investigation of the left subclavian artery.

The investigation of the vertebral artery was more complicated and as the majority of atherosclerotic lesions of the extracranial part of the posterior circulation was located at the site of its origin, this part of ultrasonic duplex scanning of the cerebral arteries needs extra attention. During the period of the first study there was no experience with ultrasonic duplex scanning of the vertebral arteries. The management of the patient and the performance of contrast arteriography was not biased by the results of ultrasonic duplex scanning. However, during the last year of the second study there was an increasing interest and confidence in ultrasonic duplex scanning and especially vertebral contrast arteriography was biased by the results of this noninvasive technique. This is reflected by the increasing percentage of the vertebral arteries with a stenosis of $50 \%$ or more at the site of the ostium (from $16.7 \%$ to $22.5 \%$ ) and the increased number of occluded vertebral arteries (from 8 to 20). An important point to emphasize here, is that only during the second study an investigation was made of the prevertebral segment of the vertebral artery along its whole length to ensure that the Doppler signals were not mistakenly recorded from another branch of the subclavian artery, such as the thyrocervical trunk. Depending on local anatomical properties the investigation of the prevertebral segment of the vertebral artery was more complicated on the left than on the right side. In general, ultrasonic duplex scanning of the ostium of the vertebral artery was impracticable in $22 \%$ of the cases. This happened in more than three quarters of the cases on the left side. In another $5 \%$ of the cases ultrasonic duplex scanning of the ostium of the vertebral artery was no problem, but the artery showed high flow or very low flow velocities. The results of the second study showed that it is inadvisable to decide upon the state of the ostium in such cases. The accuracy in detecting an occluded vertebral artery increased significantly during the four years of the study. In the first period between May 1981 and December 1982 we usually could not image an occluded vertebral artery. We were confronted with the 
same problem with hypoplastic wertebral arteries. Of the 31 vertebral arteries not found during this period only $8(26 \%)$ were occluded. During the second period between December 1982 and October 1984 this number increased to $83 \%(20 / 24)$. In this period it was not possible to image the vertebral artery, either in its prevertebral, or in its cervical segment in only $15 \%$ of the occlusions. In $35 \%$ of the cases the occluded vertebral artery was well imaged, but a Doppler measurement was impracticable. In the remaining $50 \%$ the cervical segment of the vertebral artery was imaged as well and distal collateral filling of this segment was correctly demonstrated.

Ultrasonic duplex scanning of the cervical segment of the vertebral artery was possible in almost every patient in whom we tried to perform this part of the investigation. Only in patients with marked obesity or in young children, in whom the transverse processes of the cervical vertebrae lay too close to each other, the investigation of this segment of the vertebral artery was impracticable. The assessment of coiling and kinking of the vertebral artery by osteo-arthritic spurs as well as the evaluation of atherosclerotic lesions in its cervical segment was possible in most of the patients. The influence of rotational obstruction of the vertebral artery in its atlantic and cervical segments is not yet clarified. The results of our study are too few in number to justify a conclusion about this part of the study. Contrast arteriography did not reveal an atherosclerotic obstructive lesion in the atlantic segment of the vertebral artery in any of the patients included in the second study.

In addition, the significance of ultrasonic duplex scanning of the vertebral arteries in the event of severe atherosclerotic obstructiwe disease in its intradural segment was evaluated. The interpretation of Doppler signals recorded in an intracranially occluded vertebral artery seemed to be more complicated than reported by some investigators (Ringelstein et al., 1983).

Finally, the detection of reversed flow in the vertebral artery with ultrasonic duplex scanning was no problem. There was no need for the use of special tests such as the hyperaemia test to determine the direction of flow in the vertebral arteries.

\subsection{FINAL CONCLUSIONS AND RECOMMENDATIONS}

Ultrasonic duplex scanning of the extracranial cerebral arteries, and especially the investigation of the innominate, subclavian, and vertebral arteries must not be judged as an isolated diagnostic procedure. Rather, it must be considered as a screening test which is used in combination with other diagnostic procedures, such as intravenous digital subtraction angiography to evaluate cerebral atherosclerosis. According to our experience it is possible to be adequately and reliably informed of the anatomical and physiological state of the cerebrovascular tree by a combination of diagnostic techniques invol- 
ving little hazard and morbidity and which can be performed on an outpatient basis. For the carotid arterial system it is even possible to identify a spectrum of disease involvement of the proximal internal carotid artery with acceptable accuracy. Moreover, for this part of the extracranial cerebral arteries it is also possible to differentiate between extracranially or intracranially located pressure reducing stenoses. Over the last two years intra-arterial contrast arteriography had seldom been used by us to complete diagnostic information in patients with atherosclerosis of the carotid arteries. On the other hand, conventional contrast arteriograpy was still necessary for reliable diagnostic information about the innominate, subclavian, and vertebral arteries. In our opinion, intravenous digital subraction angiography onlly gives an overall impression about this part of the cerebral arteries. More accurate information for example, about the ostium or the cervical segment of the vertebral arteries is not possible with this technique in most of the patients. Investigators like Ringelstein (1984) demonstrated that continuous wave Doppler sonography can be very helpful for a reliable non-invasive evaluation of atherosclerotic disease of the posterior circulation. Because of the limited data available, an extensive comparison between the use of continuous wave Doppler and ultrasonic duplex scanning is not possible at the present state of the art. In our opinion, anatomical information about the vessels of interest is essential in a complicated vascular bed such as the innominate-subclavian-vertebral arterial system. Therefore, we prefer the combination of ultrasonic imaging and pulsed Doppler sonography with real time spectral analysis as a routine diagnostic technique for the investigation of the innominate, subclavian, and vertebral arteries. At present continuous wave Doppler sonography is only performed in patients with a stenosis with a diameter reduction of $50 \%$ or more in the internal carotid artery, in order to be informed of the direction of flow in the peri-orbital arteries.

This study shows that in patients in whom the investigation is possible, ultrasonic duplex scanning is a usable and reasonably reliable diagnostic tool in the assessment of atherosclerotic lesions of the innominate-subclavian-vertebral arterial system. It has a high sensitivity, specificity, negative predictive value, and overall accuracy for identifying a stenosis with a diameter reduction of $50 \%$ or more in the severel segments of this part of the cerebrovascular tree. However, it has a low sensitivity and positive predictive value for the detection of a stenosis with a diameter reduction of $50 \%$ or more at the site of the origin of the right subclavian artery. Moreover, at the moment there are still too many false positive results for the detection of a stenosis with a diameter reduction of $50 \%$ or more at the site of the ostium of the vertebral artery. In most of these cases it was difficult to distinguish disturbances of the sonagrams as induced by a significant lesion of the vertebral artery from disturbances induced by high flow velocities in a vertebral artery which serves 
as a major collateral bypass of the cerebral circulation in patients with multilevel disease. In these cases non-invasive measurement of the volume of blood flow in the vertebral artery may probably give important supplementary information. However, at the moment there is no data available about the accuracy of non-invasive blood flow measurements in vertebral arteries with a high-flow state. Another problem of ultrasonic duplex scanning of the vertebral arteries is the relatively high number of patients in whom an investigation of the most proximal part and the ostium of this artery, especially on the left side, is impracticable. More appropriate transducers and signal processing techniques can be helpful in solving this problem. The use of a mechanical sector scanner with a nominal frequency of $7.5 \mathrm{MHz}$ appears to give better results for the ultrasonic imaging of the most proximal part of the vertebral arteries. However, further study is necessary to evaluate the possibilities of more recently developed duplex scanners. Moreover, the use of a transducer with a higher axial resolution has the potential power to differentiate more accurately between a hypoplastic, aplastic or occluded vertebral artery. In our second study it was not possible to image the vertebral artery, either in is prevertebral, or in its cervical segment, in only $15 \%$ of the cases with an occluded vertebral artery. The exact number of hypoplastic vertebral arteries which could not be imaged by us is not exactly known. For a more precise evaluation of the clinical significance of atherosclerotic lesions of the innominate, subclavian and vertebral arteries, ultrasonic duplex scanning of these vessels should be able, like the investigation of the carotid bifurcation, to identify a greater spectrum of disease involvement. In the near future this will be one of the challenges of ultrasonic investigation of the cerebrovascular tree. 


\section{SUMMARIES}

\subsection{ENGLISH}

A clinical study on the use of ultrasonic duplex scanning in the diagnosis of atherosclerotic obstructive disease of the innominate-subclavian-vertebral arterial system is described. It was performed on patients who also underwent contrast arteriography, the standard to which the data of ultrasonic duplex scanning was compared.

\section{Chapter 1}

This chapter is an introduction which outlines the complexity of atherosclerotic cerebrovascular disease and especially underlines the interdependence of the carotid and vertebrobasilar arterial system with relation to the vulnerability of both the supratentorial and infratentorial territories to cerebral ischaemia. The aim of the study is to determine the accuracy of ultrasonic duplex scanning of the innominate, subclavian, and vertebral arteries in patients with cerebral atherosclerosis. For this purpose the results of ultrasonic duplex scanning were compared with those of contrast arteriography. Between May 1981 and October 19842770 duplex examinations were performed in 1972 patients at the department of clinical neurophysiology of the St. Antonius Hospital, Nieuwegein, The Netherlands.

\section{Chapter 2}

In this chapter a review of the anatomy of the aortic arch, arch vessels, vertebral and basilar arteries is given. Besides the normal anatomy, anatomical anomalies are briefly described. In addition, the territories supplied with blood by these arteries and the potential collateral pathways of the vertebral arteries are discussed.

\section{Chapter 3}

The principles of cerebral autoregulation are discussed. The most important determinants of cerebral blood flow, their interaction with cerebral metabolism and involvement in the regulation of cerebral circulation are outlined. Finally the mechanisms which may play a role in the pathogenesis of transient or permanent neurological deficit are explained. 


\section{Chapter 4}

This chapter deals with atherosclerotic disease of the cerebral arteries and especially of the innominate, subclavian, and vertebral arteries. A review is given of the principle sites of atheroscleotic obstructive changes in these arteries. The clinical implication of the differences in morphology and structure of atherosclerotic lesions in the carotid and innominate-subclavian-vertebral arterial systems is briefly outlined. It is noticed that vertebrobasilar insufficiency is not a homogenous entity. It includes a variety of vascular pathologies at various locations, with diverse pathogenetic mechanisms, and with different clinical prognosis. For this reason it is not surprising that the opinions on the therapeutic results of carotid endarterectomy in this group of patients greatly vary. Finally, the clinical significance of atherosclerotic disease of the innominate, subclavian, and vertebral arteries and its interaction with obstructive disease of the carotid arteries is discussed.

\section{Chapter 5}

The clinical manifestations associated with transient ischaemia of the vertebrobasilar arterial territory usually produce a more complex profile than that found in ischaemia in the territory of the carotid arteries. Therefore, a comprehensive review of the symptomatology of transient ischaemic attacks in the vertebrobasilar territory is given in the first section of this chapter. The cervical segments of the vertebral arteries are the only parts of the cerebral vascular tree which are functionally related to degenerative osteo-arthritic change of the spinal column. Therefore, the anatomical considerations and pathogenesis of spondylosis of the cervical column and its significance in vertebrobasilar arterial insufficiency are described. The last section of this chapter deals with the pathogenesis and clinical manifestations of the subclavian steal syndrome.

\section{Chapter 6}

A short historical review is given of cerebral contrast arteriography and in particular of wertebral arteriography. In addition to this the complications of carotid and selective vertebral arteriography and the accuracy of conventional contrast arteriography and intravenous digital subtraction angiography are summarized. The next section of this chapter deals with non-invasive diagnostic techniques used in the diagnosis of atherosclerotic disease of the innominate-subclavian-vertebral arterial system. The non-imaging and imaging Doppler techniques as well as real-time ultrasonic scanning of this part of the cerebrovascular tree are discussed. Finally, the fundamental principles of ultrasonic duplex scanning are outlined. 
This chapter reviews the basic elements of the duplex scanner and associated display and recording instrumentation. During the study two types of scan heads are used, the medium focus scan head and the short focus scan head. The spatial resolution of both scan heads is evaluated and the influence of the size of the sample volumes on the quality of the Doppler signals and, consequently, the interpretation of the spectral wave forms is discussed. Finally, the technique of signal modulation-demodulation and processing is summarized.

\section{Chapter 8}

Utrasonic duplex scanning of both the carotid and the innominate-subclavian-vertebral arterial system is described. The possibilities and limitations of the investigation of each of these segments of the cerebral vascular tree is discussed. This chapter also describes the Doppler parameters used for a quantitative assessment of atherosclerotic obstructive lesions in the innominate, subclavian, and vertebral arteries.

\section{Chapter 9}

This chapter describes our results of ultrasonic duplex scanning of the innominate, subclavian, and vertebral arteries. For each of these vessels the results of the non-invasive technique are compared with the results of contrast arteriography and they are reviewed separately. First, however, the method of assessment of the percentage of stenosis used in this study and the statistical analysis of the data are described.

Two studies form the basis of the evaluation of the data of ultrasonic duplex scanning. During the first study we only investigated the proximal segment of the subclavian and vertebral arteries. During the second study we also investigated the innominate artery and the cervical segment of the vertebral artery. In 14\% of the patients a non-invasive investigation of the innominate artery was impracticable and in $22 \%$ of the cases it was not possible to decide upon the state of the origin of the vertebral artery. Mostly a deep location of the relevant part of the cerebral vasculature made the non-invasive investigation impracticable. Therefore, the hereby mentioned accuracy only counts in those cases in whom the examination was succesful. The accuracy of ultrasonic duplex scanning of the innominate and subclavian arteries as well as the ostium of the vertebral arteries is expressed in sensitivity, specificity, positive predictive value, negative predictive value, and overall accuracy. Most of these parameters show that ultrasonic duplex scanning of these 
segments of the cerebrovascular tree can be used as a reliable screening test in patients with cerebral atherosclerosis. However, the sensitivity for identifying a stenosis with a diameter reduction of $50 \%$ or more at the site of the origin of the right subclavian artery is too low $(0.43)$. Moreover, the positive predictive value of ultrasonic duplex scanning of the ostium of the vertebral artery $(0.65)$ needs improvement. A possible explanation is given for the false negative and false positive test results of ultrasonic duplex scanning for each of the separate arteries. In particular a high-flow or low-flow state of the vertebral artery makes a reliable diagnosis of an obstructive lesion in this artery very difficult. In addition, the results of the second study show that in experienced hands the differentation between hypoplasia and occlusion of the vertebral artery is very well possible. The investigation of the cervical segment of the vertebral artery is only performed if the patient had a history of vertebrobasilar insufficiency or if it was requested by the referring physician. Especially for this part of the vertebral artery the relation between osteo-arthritic spurs caused by cervical spondylosis and displacement or obstruction of the vertebral artery is evaluated. Although the number of patients with abnormalities in the cervical segment of the vertebral artery is small, ultrasonic duplex scanning of the cervical segment appeared to be a reliable test. The significance of ultrasonic duplex scanning of the vertebral artery in the event of a severe, obstructive lesion in the intradural segment of this artery is also evaluated. The interpretation of the morphology of the sonagrams recorded in an intracranially occluded vertebral artery appear to be more complicated than in the carotid system. Probably this is the result of a greater variation in the territory supplied with blood by an intracranially occluded vertebral artery. Finally, the results of ultrasonic duplex scanning of the vertebral arteries in patients with a subclavian or innominate steal syndrome are reviewed. For this part of the study we also use the data of intravenous digital subtraction angiography.

\section{Chapter 10}

This chapter outlines the potential power of intravenous digital subtraction angiography and ultrasonic imaging in the assessment of atherosclerotic disease and in particular of atherosclerosis of the cerebral arteries. In addition to this, the impact of ocular pneumoplethysmography, intravenous digital subtraction angiography and ultrasonic duplex scanning on the management of patients with atherosclerosis of the cerebral arteries in our hospital is discussed. "The consistent use of these three diagnostic procedures as a battery of screening tests gives adequate and reliable information about the state of the cerebral arteries extracranially and intracranially in most of the patients. The need for conventional contrast arteriography in patients with atherosclerosis 
of the cerebral arteries has significantly decreased over the last two years. In addition this chapter gives a review of the possibilities and limitations of ultrasonic duplex scanning of the innominate, subclavian and vertebral arteries. Finally, some recommendations and future expectations are given for a more accurate and complete non-invasive investigation of the innominatesubclavian-vertebral arterial system.

\subsection{DUTCH}

De klinische bruikbaarheid van het duplexonderzoek ten behoeve van patiënten met atherosclerose van de aa. anonyma, subclavia en vertebralis wordt beschreven. De in deze studie opgenomen patiënten hebben tevens contrastarteriografie ondergaan. De resultaten van het duplexonderzoek worden vergeleken met de uitkomsten van arteriografie.

\section{Hoofdstuk 1}

In dit hoofdstuk wordt het complexe karakter van atherosclerose van de hersenvaten uiteengezet. Hierbij wordt de nadruk gelegd op de onderlinge afhankelijkheid van het carotis- en vertebralissysteem met betrekking tot ischaemie van zowel supratentoriële als infratentoriële hersenstructuren. Het doel van de studie is het bepalen van de nauwkeurigheid van het duplexonderzoek van de aa. anonyma, subclavia en vertebralis bij patiènten met atherosclerose. Hiertoe worden de resultaten van het duplexonderzoek vergeleken met de uitkomsten van contrastarteriografie. In de periode tussen mei 1981 en oktober 1984 zijn op de afdeling Klinische Neurofysiologie van het St. Antonius Ziekenhuis te Nieuwegein, Nederland bij 1972 patiënten in totaal 2770 duplexonderzoeken uitgevoerd.

\section{Hoofdstuk 2}

Dit hoofdstuk geeft een overzicht van de anatomie van de aortaboog, boogvaten, a. vertebralis en a. basilaris. Naast de normale anatomie worden tevens een aantal aangeboren anatomische afwijkingen in het kort behandeld. Bovendien worden de verzorgingsgebieden van deze bloedvaten en de potentiele collaterale circulatie van de aa. vertebrales beschreven.

\section{Hoofdstuk 3}

De grondbeginselen van de cerebrale autoregulatie worden besproken. De belangrijkste factoren die de doorbloeding van de hersenen regelen en hun interactie met de hersenstofwisseling alsmede hun invloed op de regulatie van 
de hersencirculatie worden uiteengezet. Tenslotte worden de verschillende mechanismen die een rol kunnen spelen bij het ontstaan van voorbijgaande of blijvende neurologische uitvalsverschijnselen behandeld.

\section{Hoofdstuk 4}

Dit hoofdstuk behandelt de atherosclerose van de extracraniële cerebrale arteriën en in het bijzonder van de aa. anonyma, subclavia en vertebralis. Er wordt een overzicht gegeven van de meest belangrijke plaatsen van atherosclerotische vaatwandweranderingen in de laatst genoemde bloedvaten. De klinische betekenis van het verschil in morfologie en structuur van atherosclerotische letsels van het carotis- en het anonyma-subclavia-vertebralis-systeem wordt besproken. Het syndroom van de vertebrobasilaire insuffiëntie is geen klinische eenheid. Het bestaat uit een verzameling van op meerdere plaatsen gelocaliseerde vaatwandafwijkingen met verschillende pathogenetische mechanismen en veelal uiteenlopende prognose. Het is dan ook begrijpelijk, dat de opvattingen over het nuttig effect van carotisendarteriëctomie bij deze groep patiënten nogal variëren. Tenslotte wordt in dit hoofdstuk de klinische betekenis van atherosclerose van de aa. anonyma, subclavia en vertebralis en in het bijzonder de interactie met obstruerende afwijkingen van de a. carotis besproken.

\section{Hoofdstuk 5}

De klinische verschijnselen die kunnen optreden bij een tijdelijke doorbloedingsstoornis van het vertebrobasilaire systeem zijn in het algemeen veel complexer van aard dan de verschijnelen die worden waargenomen bij een ischaemie in het carotisgebied. In het eerste deel van dit hoofdstuk wordt daarom een uitgebreid overzicht gegeven van de symptomatologie van voorbijgaande doorbloedingsstoornissen van het vertebrobasilaire gebied. Het cervicale segment van de a. vertebralis is het enige deel van de cerebrale vaatboom dat in functionele relatie staat tot de halswervelkolom. De anatomie en de pathogenese van de cervicale spondylose en hun betekenis voor de vertebrobasilaire insufficièntie worden besproken. Het laatste deel van dit hoofdstuk behandelt de pathogenese en de klinische verschijnselen van het "subclavian steal" syndroom.

Hoofdstuk 6

Er wordt een kort, historisch overzicht gegeven van de cerebrale contrastarteriografie in het bijzonder van de vertebralisarteriografie. Tevens worden de complicaties van selectieve arteriografie van de a. carotis en a. vertebralis 
besproken. De nauwkeurigheid van zowel de conventionele contrastarteriografie als van de intraveneuze digitale substractie-angiografie worden samengevat. Daarna worden de verschillende niet-invasieve methoden van ultrageluidsdiagnostiek van het anonyma-subclavia-vertebralis-systeem besproken. Zowel de conventionele als de beeldvormende Doppler-technieken en de "real-time" tweedimensionale echografie worden behandeld. Tenslotte worden de grondbeginselen van het duplexonderzoek uiteengezet.

\section{Hoofdstuk 7}

Dit hoofdstuk geeft een overzicht van de verschillende onderdelen van de meetapparatuur en de daarbij behorende registratie-apparatuur. Tijdens deze studie zijn twee typen transducers gebruikt: de "mid-focus" en de "short focus" transducer. De ruimtelijke eigenschappen van beide transducers zijn bepaald en de invloed van de grootte van het "sample volume" op de kwaliteit van de Doppler-signalen en derhalve op de interpretatie van de sonogrammen wordt besproken. Tevens wordt de techniek van modulatie-demodulatie en signaalverwerking in het kort behandeld.

\section{Hoofdstuk 8}

Zowel het duplexonderzoek van het carotissysteem als van het anonymasubclavia-vertebralis-systeem wordt beschreven. Voor ieder onderdeel van het extracraniële deel van de cerebrale vaatboom worden de beperkingen van het duplexonderzoek genoemd. In dit hoofdstuk worden tevens de parameters gedefinieerd, die gebruikt worden bij de kwantitatieve bepaling van de mate wan stenose van respectievelijk de a. anonyma, a. subclavia en a. vertebralis.

\section{Hoofdstuk 9}

Dit hoofdstuk beschrijft onze resultaten van het duplexonderzoek van de aa. anonyma, subclavia en vertebralis. Voor ieder van deze drie arteriên worden de uitkomsten van het duplexonderzoek vergeleken met de resultaten van de contrastarteriografie. Eerst echter bespreken we de wijze waarop de mate van diameterreductie op het arteriogram wordt bepaald en de statistische analyse van de gegevens.

Twee studies wormen de basis voor het bepalen van de bruikbaarheid en nauwkeurigheid van het duplexonderzoek. Gedurende de eerste studie is door ons alleen het proximale deel van de a. subclavia en de a. vertebralis onderzocht. Gedurende de tweede studie zijn ook de a. anonyma en het cervicale segment van de a. vertebralis bij het onderzoek betrokken. De hier gepresenteerde betrouwbaarheid van het ondlerzoek geldt alleen indien de desbetref- 
fende arterie onderzocht kan worden. In 14\% van de patiënten kan geen uitspraak gedaan worden over de a. anonyma en in $22 \%$ van de onderzochte vaten kan de a. vertebralis ter plaatse van het ostium niet worden beoordeeld. Meestal maakt een te diepe ligging van het bloedvat het onderzoek dan onmogelijk. De nauwkeurigheid van het duplexonderzoek wordt uitgedrukt in respectievelijk de sensitivteit, specificiteit, positief voorspellende waarde, negatief voorspellende waarde en de algehele nauwkeurigheid. De meeste van de hier genoemde parameters tonen aan, dat het duplexonderzoek van de verschillende onderdelen van het anonyma-subclavia-vertebralis-systeem een betrouwbare test is. De sensitiviteit voor het aantonen van een stenose van meer dan $50 \%$ diameterreductie ter plaatse van het ostium van de rechter a. subclavia is echter nog te laag $(0,43)$. Bovendien behoeft de positief voorspellende waarde van het duplexonderzoek van het ostium van de a. vertebralis $(0,65)$ verbetering. Voor ieder onderdeel van het anonyma-subclavia-vertebralis-systeem wordt een mogelijke verklaring gegeven voor zowel de fout negatieve als de fout positieve resultaten van het onderzoek. Zo blijkt, dat zowel zeer hoge als zeer lage stroomsnelheden in een a. vertebralis een nauwkeurige beoordeling van de toestand van dit vat ter plaatse van het ostium ernstig bemoeilijken. Tevens blijkt dat tijdens de tweede studie, bij toenemende ervaring van de onderzoekers, het onderscheid tussen een hypoplasie en een occlusie van de a. vertebralis duidelijk is verbeterd. Het onderzoek van het cervicale segment van de a. vertebralis is alleen bij die patiënten uitgevoerd bij wie de anamnese zou kunnen wijzen op een vertebrobasilaire insufficiëntie of indien dit deel van het onderzoek nadrukkelijk werd aangevraagd. Voor dit segment van de a. vertebralis is de relatie tussen cervicale spondylose en verdringing, respectievelijk obstructie van de a. vertebralis nader onderzocht. Hoewel het aantal patiënten met afwijkingen in het cervicale segment klein is, blijkt het duplexonderzoek van dit deel van de a. vertebralis betrouwbaar te zijn. De betekenis van het duplexonderzoek van de a. vertebralis in het geval van een ernstige, obstruerende afwijking in het intradurale segment van deze arterie is eveneens bepaald. Waarschijnlijk als gevolg van de relatief grote variatie in het verzorgingsgebied van een intracranieel afgesloten a. vertebralis blijkt de interpretatie van de meer proximaal geregistreerde sonogrammen tot fout negatieve resultaten aanleiding te kunnen geven. Tenslotte worden de resultaten van het duplexonderzoek van de a. vertebralis bij patiënten met een "subclavian steal" syndroom samengevat. Voor dit deel van de studie zijn ook de gegevens van de intraveneuze digitale subtractie-angiografie gebruikt.

Hoofdstuk 10

Dit hoofdstuk schetst de mogelijkheden van de intraveneuze digitale subtractie-angiografie en de ultrasonische beeldvorming, wooral ten behoeve van 
de diagnostiek van atherosclerose van de hersenvaten. Daarna wordt de betekenis van de oculopneumoplethysmografie, de intraveneuze digitale subtractie-angiografie en het duplexonderzock voor het beleid met betrekking tot patiënten met cerebrale atherosclerose in ons ziekenhuis besproken. $\mathrm{Bij}$ consequent gebruik van deze drie methoden van onderzoek blijkt men voldoende en betrouwbare informatie te kunnen verkrijgen over de toestand van de cerebrale vatboom, zowel intracranieel als extracranieel. Hierdoor is de laatste twee jaar de behoefte aan conventionele contrastarteriografie bij patiënten met cerebrale atherosclerose beduidend afgenomen. Tevens worden de mogelijkheden en de beperkingen van het duplexonderzoek van de aa. anonyma, subclavia en wertebralis nog eens samengevat. Tot slot worden er een aantal suggesties gegeven, die in de nabije toekomst het niet-invasieve onderzoek van het anonyma-subclavia-vertebralis-systeem zouden kunnen verbeteren.

\subsection{FRENCH}

Le travail clinique présenté décrit l'emploi de l'echotomographie couplée à un Doppler pulsé dans le diagnostique des lésions athéromateuses obstructives du tronc brachiocéphalique et des artères sous-clavières et vertébrales. L'étude concerne des malades ayant subi une angiographie artérielle considerée comme standard de comparaison pour le duplexscanning (echotomographie couplée au Doppler pulsé).

\section{Chapitre I}

Dans ce chapitre la complexité de l'athéromatose cérébrovasculaire est décrite et en particulier l'interdépence des systèmes artériels carotidiens et vertébrobasilaires dans la vulnérabilité tant des territoires supratentoriels que des territoires infratentoriels. L'étude se pose comme but de définir la fiabilité du duplexscanning dans la définition des localisations athéromateuses du tronc brachiocéphalique et des artères sous-clavières et vertébrales. A cette fin on a comparé les résultats du duplexscanning avec les résultats des examens angiographiques. De mai 1981 à octobre 19842770 examens au duplexscanming ont été réalisés chez 1972 malades dans le département de neurophysiologie clinique de l"hôpital St. Antoine à Nieuwegein, Hollande.

\section{Chapitre 2}

Ce chapitre donne un résumé de l'anatomie de la crosse aortique, de la gerbe aortique et des artères vertébrales et basilaire. Les anomalies anatomiques sont décrites brièvement. On décrit également les territoires irrigués par ces artères et les voies collatérales vers le système vertébrobasilaire. 
Dans ce chapitre on résume les principes de l'autorégulation cérébrale. On revoit les principaux déterminants du débit cérébral et leur interaction tant avec le métabolisme cérébral qu'avec l'autorégulation de la perfusion cérébrale. On explique les méchanismes qui peuvent jouer un rôle dans la pathogénèse des déficits neurologiques temporaires ou permanents.

\section{Chapitre 4}

Dans ce chapitre la maladie athéromateuse des artères cérébrales est revue et en particulier celle du tronc brachiocéphalique et des artères sous-clavières et vertébrales. Les localisations prédominantes des lésions athéromateuses sont revues. Les implications cliniques des différences de la morphologie et de la structure pathologiques entre les lésions carotidiennes et celles du système artériel tronc brachiocéphalique, et des artères sous-clavières et vertébrales sont resumées. L'insuffísance vertébrobasillaire ne se présente pas comme une entité homogène; plusieurs localisations pathologiques et différents méchanismes pathologéniques peuvent intévenir et être associés à des pronostic très différents. Il n'est donc pas étonnant que les résultats de l'endartérectomie carotidienne en présence de symptomes d'insulfisance vertébrobasilaire soient très variables. La signification clinique des atteintes du trone brachiocéphalique et des artères sous-clavières et vertébrales est discutée dans sa relation avec les lésions obstructives des carotides.

\section{Chapitre 5}

Les manifestations cliniques de l'ischémie passagère dans le territoire vertébrobasilaire se présentent d'une façon plus complexe que celles du territoire carotidien. Elle sont présentées d'une façon détaillée dans la première partie de ce chapitre. Parmi les artères cérébropétales seulement les segments cervicaux des artères vertébrales sont en contact avec la colonne cervicale; leur fonction peut donc être influencée par des lésion d'ostéo-arthrose. Des considérations anatomiques et pathogéniques concernant la spondylose cervicale sont présentées afin d'éclaircir leur rôle dans l'insuffisance vertébrobasilaire. La fin de ce chapitre résume la pathogénèse et les manifestations cliniques du syndrome du vol sous-clavier.

\section{Chapitre 6}

Dans ce chapitre on revoit l'historique de l'angiographie cérébrale et de l'angiographie vertébrobasilaire en particulier. On revoit les complications de 
l'artériographie sélective carotidienne et vertébrale ainsi que le problème de la fiabilité de l'angiographie conventionelle et de l'angiographie intraveineuse numérisée. La seconde partie de ce chapitre est consacrée aux techniques de diagnostic non invasif dans l'athéromatose du système brachiocéphalique, sous-clavier et vertêbral. On discute les méthodes Dopplers à information hémodynamique et les Dopplers à reconstitution morphologique ainsi que les echotomographie en temps réel, dans le contexte des lésions des artères afférentes du système vertébrobasilaire. La dernière partie concerne les principes fondamentaux de l'echotomographie en temps réel associée à l'ultrasonographie Doppler (duplexscanning).

\section{Chapitre 7}

Dans ce chapitre les différentes composantes du duplexscanner sont décrites ainsi que l'appareillage associé pour la visualisation et l'enregistrement. Durant cette étude deux capteurs echotomographique différents ont été employés, respectivement un capteur à foyer moyen et un capteur à foyer court. La résolution spatiale des deux capteurs a été évaluée ainsi que l'influence du volume analysé par le Doppler pulsé sur la qualité des signaux Dopplers et par conséquent sur l'interprétation de l'analyse spectrale du signal Doppler. La technique de modulation et de démodulation dans l'élaboration du signal est résumée.

\section{Chapitre 8}

Dans ce chapitre on décrit le duplexscanning des artères carotidiennes et de l'axe brachiocéphalique, sous-clavier et vertébral. Tant les possibilités que les limites de l'application dans chaque domaine sont discutées. On présente également les paramètres Dopplers employés pour l'analyse quantitative des lésions athéromateuses sténosantes de l'axe brachiocéphalique, sous-clavier et vertébral.

\section{Chapitre 9}

Dans ce chapitre nous décrivons nos résultats du duplexscanning de l'artère brachiocéphalique et des artères sous-clavières et vertébrales. Pour chaque artère nous comparons les résultats de cette technique non-invasive avec celle de l'angiographie. Avant tout, on précise la méthode d'appréciation du pourcentage de sténose, ainsi que la méthode d'analyse statistique employée. L'évaluation du duplexscanning s'est faite en deux phases: dans un premier temps uniquement les artères sous-clavières et la partie initiale des artères vertébrales furent étudiées; dans la suite l'artère brachiocéphalique et les 
segments cervicaux de l'artère vertébrale furent également analysées. La fiabilité du duplexscanning dans la détection des lésions de l"artère brachiocé. phalique, ainsi que des artères sous-clavières et des lésions ostiales de l'artère vertébrale est exprimée en sensitivité et sensibilité, en valeur prédictive positive et négative ainsi qu'en fiabilité générale. La plupart de ces paramètres démontrent que le duplexscanning est suffisamment fiable pour constituer une exploration de triage dans les malades avec des lésions cérébrovasculaires athéromateuses. Toutefois, la sensitivité pour identifier les lésions de $50 \%$ ou plus à l'origine de la sous-clavière droite est trop basse (0.43); également, la valeur prédictive positive pour les lésions ostéales vertébrales laisse à désirer (0.65). On examine les explications possible pour les tests faux-positifs ou faux-négatifs dans le duplexscanning des différentes artères. En particulier, c'est dans les artères vertébrales à très gros ou à très bas débit que le diagnostic des lésions sténosantes des artères vertébrales devient très difficile. Dans la seconde phase de l'étude on a pu démontrer que des opérateurs entraînés peuvent très bien distinguer une hypoplasie d'une occlusion de l'artère vertébrale. Cette étude du segment cervical de l'artère vertébrale est executé uniquement en cas de symptomatologie vertébrobasilaire ou sur demande explicite du médecin traîtant. C'est précisement dans ce segment cervical que la relation avec des lésions d'ostéo-arthrose cervicale pouvant influencer la fonction vertébrale par déplacement ou compression a été étudiée. Malgré le nombre limité des malades présentant des anomalies du segment cervical, le duplexscanning s'est avéré une méthode fiable.

On a également étudié la valeur du duplexscanning dans les lésions sévères du segment intradural des artères vertébrales. L'interprétation du signal Doppler en cas d'occlusion intracranienne de l'artère vertébrale s'avère plus difficile que pour l'artère carotidienne. Cela peut s'expliquer par une plus grande variabilité dans le territoire irrigué par l'artère vertébrale obstruée. En fïn de chapitre les résultats du duplexscanning des artères vertébrales en cas de vol brachiocéphalique ou sous-clavier, sont présentés. Pour cette partie les résultats de l'angiographie intraveineuse numérisées ont également été acceptés.

\section{Chapitre 10}

Dans ce chapitre le rôle possible de l'angiographie intraveineuse numérisées, et du duplexscanning dans le diagnostic des lésions athéromateuses des artères cérébropétales en particulier est décrit. Le rôle joué dans notre hôpital par l'oculopneumopléthysmographie selon Gee, par l'angiographie intraveineuse numérisées et par le duplexscanning, dans le traitement des malades présentant des lésions des artères cérébrales est discuté. L'emploi systếmatique de ces trois méthodes diagnostiques en combiné, donne des information fiables sur les lésions extra- et intracraniennes présentes dans la plupart de nos 
malades. Le recours à l'angiographie intra-artérielle a très fortement díminué les deux dernières années. Ce chapitre résume les possibilités et les limitations du duplexscanning de l'artère brachiocéphalique et des artères sous-clavières et vertébrales. En fin de chapitre quelques recommandations sont formulées qui pourraient améliorer dans un avenir immédiat le diagnostic non-invasif de l'axe brachiocéphalique, sous-clavier et wertébral.

\subsection{GERMAN}

Die klinische Brauchbarkeit der Duplex-Sonographie zum Besten der Patienten mit Atherosklerose der A. anonyma, A. subclavia und A. vertebralis wird beschrieben. Die in dieser Arbeit aufgenommen Patienten haben auch Kontrast-Arteriographie erfahren. Die Resultate der Duplex-Sonographie werden verglichen mit die Ergebnisse der Arteriographie.

\section{Kapitel 1}

Dieses Kapitel befasst sich mit die komplexe Eigenart von Atherosklerose der hirnzuführende Gefässe. Die gegenseitige Abhängigkeit der Karotis- und Vertebralis-Systeme in Beziehung zu Ischämia sowohl der supratentorielle als der infratentorielle Gehirnstrukture wird betont. Der Ziel dieser Arbeit ist die Erfassung der Genauigkeit der Duplex-Sonographie der A. anonyma, A. subclavia und A. vertebralis bei Patienten mit Atherosklerose. Zu diesem Ziel werden die Resultate der Duplex-Sonographie verglichen mit die Ergebnisse won Kontrast-Arteriographie. In die Abteilung für klinische Neurophysiologie des St. Antonius Hospital in Nieuwegein, Niederlände, sind in die Zeitspanne zwischen Mai 1981 und Oktober 1984 bei 1972 Patienten insgesamt 2770 Duplex-Sonographien durchgeführt worden.

\section{Kapitel 2}

Dieses Kapitel gibt eine Übersicht der Anatomie von den Aorta-Bogen, die Bogengefässe, der $\mathrm{A}$. vertebralis und der $\mathrm{A}$. basilaris. Neben die normale Anatomie werden einige angeborene Abweichungen kurz behandeit. Ausserdem werden die Versorgunsgebiete dieser Gefässe und die potentielle kollaterale Zirkulation der $\mathrm{Aa}$. vertebrales beschrieben.

\section{Kapitel 3}

Die Prinzipien der zerebrale Autoregulation wird besprochen. Die wichtigste Faktore die die Durchblutung des Gehirnes regulieren, deren Interaktion 
mit dem Stoffwechsel des Gehirnes und deren Einfluss auf die Regulation der Gehirn-Zirkulation werden dargelegt. Schliesslich werden die unterschiedliche Mechanismen die eine Rolle spielen können beï der Entstehung von vorübergehende oder bleibende neurologische Ausfall-Symptome behandelt.

\section{Kapitel 4}

Dieses Kapitel behandelt den Atherosklerose der extrakranielle zerebrale Arterien und besonders der A. anonyma, A. subclavia und A. vertebralis. Eine Übersicht wird gegeben von der wichtigsten Stellen von atherosklerotischen Gefässwand-Änderungen in der letzterwähnte Gefässe. Die klinische Bedeutung des Unterschiedes in Morphologie und Struktur von atherosklerotischen Schäden des Karotis- und des Anonyma-Subclavia-Vertebralis-Systems wird besprochen. Das Syndrom der vertebrobasiläre Insuffizienz ist keine klinische Einheit. Es besteht aus einer Sammlung von an mehreren Stellen lokalisierter Abweichungen der Gefässwände mit unterschiedliche pathogenetische Mechanismen und oft sehr verschiedene Prognosen. Es ist dann auch leicht zu verstehen dass die Auffassungen über die nützliche Wirkung von Karotisendarteriectomie bei dieser Gruppe von Patienten ziemlich variieren. Schliesslich wird in diesem Kapitel die klinische Bedeutung von Atherosklerose der $\mathbf{A}$. anonyma, A. subclavia und $\mathbf{A}$. vertebralis und besonders die Interaktion mit obstruierende Abweichungen der A. carotis besprochen.

Kapitel 5

Die klinische Symptome die auftreten können bei eine zeitweilige Durchblutingsstörung des vertebrobasiläre Systems sind im Allgemeinen viel komplexer geartet als die Symptome die beobachtet werden bei eine Ischämia des Karotis-Gebietes. Im ersten Teil dieses Kapitels wird deshalb eine ausführliche Ubersicht gegeben der Symptomatologie von vorübergehenden Durchblutungsstörungen des vertebrobasiläre Gebietes. Das zervikale Segment der A. vertebralis ist das einzige Teil des zerebralen Systems dass in funktioneller Beztehung steht zum Halswirbelsäule. Die Anatomie und die Pathogenese der zervikalen Spondylose und deren Bedeutung für der vertebrobasiläre Insuffizienz werden besprochen. Das letzte Teil dieses Kapitels behandelt die Pathogenese und die klinische Symptome des "subclavian steal" Syndroms.

\section{Kapitel 6}

Es wird eine kurze geschichtliche Übersicht gegeben von der zerebrale Kontrast-Arteriographie, ins besonders der Vertebralis-Arteriopgraphie. Zugleich werden die Komplikationen von selektive Arteriographie der A. carotis und 
der A. vertebralis besprochen. Die Genauigkeit von sowohl der konventionellen Kontrast-Arteriographie als von der intravenöse digitale Subtraktions-Arteriographie werden zusammengefasst. Darauf werden die unterschiedliche nicht-invasive Methoden der Ultra-Sonodiagnostik des Anynoma-SubclaviaVertebralis-Systems besprochen. Sowohl die konventionelle als die Bildformende Dopplertechnike und die "real-time"' zweidimensionelle Echographie werden behandelt. Schliesslich werden die Prinzipen der Duplex-Sonographie auseinandergesetzt.

\section{Kapitel 7}

Dieses Kapitel gibt eine Übersicht der untderschiedliche Elemente der Messapparatur und der angehörige Registrationsapparatur. Während diese Arbeit sind zwei Typen von Transducern verwendet worden: der "'mid-focus" und der "short focus" Transducer. Die räumliche Eigenschafte von beide Transducern sind bestimmt worden und der Einfluss der Grösse von das "sample volume" auf die Qualität der Doppler-Signale und deshalb auf die Interpretation der Sonogramme wird besprochen. Zugleich wird die Technik der Modulation-Demodulation und Signalbearbeitung kurz behandelt.

Kapitel 8

Die Duplex-Sonographie des Karotissystems sowie die des Anonyma-Subclavia-Vertebralis-Systems werden beschrieden. Für jeden Einzelteil des extrakraniellen Teils des zerebralen Systems werden die Beschränkungen der Duplex-Sonographie genennt. Zugleich werden in diesem Kapitel die Parameter definiert, die verwendet werden bei der quantitativen Bestimmung der Grad der Stenose von beziehungsweise der A. anonyma, A. subclavia und A. vertebralis.

Kapitel 9

Dieses Kapitel beschreibt unsere Ergebnisse der Duplex-Sonographie der A. anonyma, A. subclavia und A. vertebralis. Für jede dieser drei Arterien werden die Ergebnisse der Duplex-Sonographie verglichen mit der Resultate der Kontrast-Arteriographie. Zuerst besprechen wir die Art und Weise wie der Grad der Diameterreduktion auf das Arteriogramm bestimmt wird und die statistische Auswertung der Daten.

Zwei Studien formen die Grundlegung zur Bestimmung der Brauchbarkeit und Genauigkeit der Duplex-Sonographie. Während des ersten Studiums haben wir nur das proximale Teil der A. subclavia und der A. vertebralis untersucht. Während des zweiten Studiums sind auch die A. anonyma und das 
zervikale Segment der A. vertebralis in der Untersuchungen bezogen. Die hier präsentierte Genauigkeit der Untersuchung gilt nur wann die diesbezügliche Arterie untersucht werden kann. Bei 14\% der Patienten kann nicht entschieden werden über die A. anonyma und in $22 \%$ der untersuchten Gefässe kann die A. vertebralis zur Stelle des Ostiums nicht beurteilt werden. Meistens macht eine zu tiefe Lage des Gefässes die Untersuchung dann unmöglich. Die Genauigkeit der Duplex-Sonographie wird ausgedrückt in beziehungsweise die Sensitivität, die Spezifität, der positiv vorhersagende Wert, der negativ vorhersagende Wert und die allgemeine Genauigkeit. Die meiste der hier genennte Parameter zeigen, dass die Duplex-Sonographie der unterschiedliche Einzelteile des Anonyma-Subclavia-Vertebralis-Systems ein zuverlässiger Test ist. Die Sensitivität für dass Zeigen einer Stenose von mehr als $50 \%$ Diameterreduktion zur Stelle des Ostiums der rechten A. subclavia ist aber noch zu niedrig (0.43). Ausserdem bedürft der positiv vorhersagende Wert der Duplex-Sonographie des Ostiums der A. vertebralis $(0.65)$ noch einige Verbesserung. Für jedes Einzelteil des Anonyma-Subclavia-Vertebralis-Systems wird eine mögliche Erklärung gegeben für sowohl die falsch-negative als die falsch-positive Ergebnisse der Untersuchung. So ergebt sich zum Beispiel dass sowohl sehr hohe als sehr niedrige Fliessgeschwindigkeiten in einer A. vertebralis eine genaue Beurteiling des Zustand dieses Gefässes zur Stelle des Ostiums stark erschweren. Zugleich ergibt sich dass während des zweiten Studiums, mit zunehmender Erfahrung der Untersucher, das Unterschied zwischen eine Hypoplasie und eine Okklusion der A. vertebralis deutlich verbessert ist. Die Untersuchung des zervikalen Segments der A. vertebralis ist nur bei solche Patienten durchgeführt bei welche die Anamnese deuten könnte auf eine vertebrobasiläre Insuffizienz oder wann nachdrücklich gebeten würde om diesen Teil der Untersuchung. Für dieses Segment der A. wertebralis ist die Beziehung zwischen zervikale Spondylose und Verdrängung, beziehungsweise Obstruktion der $\mathrm{A}$. vertebralis näher untersucht worden. Obwohl die Anzahl der Patienten mit Abweichungen in dem zervikalen Segment klein ist, ergebt sich die Duplex-Sonographie dieses Teiles der A. vertebralis als zuverlässig. Die Bedeutung der Duplex-Sonographie der A. vertebralis im Fall einer ernsthafter obstruierender Abweichung in das intradurale Segment dieser Arterie ist ebenfalls bestimmt worden. Wahrscheinlich als Folge der relativ grosse Variation in dem Versorgungsgebiet einer intrakraniell abgeschlossener $\mathbf{A}$. vertebralis ergebt sich, dass die Interpretation von mehr proximal registrierten Sonogramme falsch-negative Ergebnisse veranlassen könmen. Schliesslich werden die Ergebnisse der Duplex-Sonographie $\operatorname{der}$ A. vertebralis bei Patienten mit einem "subclavian steal" Syndron zusammengefasst. Für dieses Teil der Arbeit sind auch Daten der intravenösen digitalen Subtraktions-Angiographie benutzt worden. 
Diese Kapitel skizziert die Mögligkeiten der intravenöse digitale Subtraktions-Angiographie und die Ultrasone Bildgestaltung, speziell behufs der Diagnostik von Atherosklerose der Gehirnfässe. Nachdem wird die Bedeutung der Okulopneumoplethysmographie, der intravenöse digitale Subtraktions-Angiographie und der Duplex-Sonographie für die Umsicht in Beziehung der Patienten mit zerebralen Atherosklerose in unserum Hospital besprochen. Bei consequenter Benutzung dieser drei Untersuchungsmethoden ergebt sich dass man genügende und zuverlässige Information bekommt über den Zustand des zerebralen Systems, sowohl intrakraniell als extrakraniell. Hierdurch ist die letzte zwei Jahre der Bedarf an konventionelle KontrastArteriographie bei Patienten mit zerebralen Atherosklerose bedeutend verringert. Zugleich werden die Möglichkeiten und die Beschränkungen der Duplex-Sonographie der A. anonyma, A. subclavia und A. vertebralis noch einmal zusammengefasst. Zum Schluss werden enige Suggestionen gegeben, die in naher Zukunft die nicht-invasive Untersuchung des Anonyma-Subclavia-Vertebralis-Systems verbessersn könnten.

\subsection{INDONESIAN}

Pada penelitan klinis telah dilakukan perbandingan penggunaan skan ultrasonik dupleks dengan arteriografi kontras dalam mendiagnosa penyakit atherosklerosis yang menyebabkan sumbatan dari sistim arteri innominatasubklavia-vertebralis.

\section{Bab ॥}

Bab pendahuluan yang menggambarkan ke-aneka ragaman penyakit atherosklerosis pembuluh darah otak, khususnya mengutamakan sifat saling mempengaruhi dari sistim carotis dengan arteri vertebro-basilaris di daerah pertemuan supra-infra tentorial dalam iskemi otak. Tujuan penelitan untuk menentukan ketepatan skan ultrasonik dupleks dari arteri innominata, subklavia dan vertebralis yang mengalami atherosklerosis. Untuk tujuan ini, hasil skan ultrasonik dupleks telah dibandingkan dengan arteriografi kontras. Dari bulan Mei 1981 sampai bulan oktober 1984, telah dilakukan 2770 pemeriksaan dupleks terhadap 1972 penderita di departemen neurofisiologi klinis, R.S. St. Antonius, Nieuwegein, Negeri Belanda. 
Pembahasan anatomi arkus aorta berserta pembuluhnya, arteri vertebralis dan basilaris. Selain yang normal, anomali anatomis dilukiskan secara singkat. Sebagaj tambahan telah dibahas daerah yang diperdarahi pembuluh darah tsb. diatas, serta aliran kolateral yang penting dari arteri vertebralis.

\section{Bab 3}

Telah dibahas prinsip otoregulasi otak besar. Terutama ditekankan pada pentingnya aliran darah ke otak besar, hubungannya dengan metabolisme otak serta keterlibatannya dalam pengaturan peredaran darah otak besar. Akhirnya telah dijelaskan mekanisme yang mungkin berperan sebagai patogenesa defisit neurologis yang bersifat sementara ataupun menetap.

\section{Bab 4}

Bab ini berhubungan dengan penyakit atherosklerosis arteri serebralis, khususnya arteri innominata, subklavia dan vertebralis. Telah dibahas prinsip terjadinya atherosklerosis yang menyumbat pembuluh darah tsb. diatas. Secara garis besar dan singkat telah diterangkan perbedaan bentuk dan struktur dari lesi atherosklerosis pada sistim carotis dengan sistim innominata, subklavia, vertebralis dalam pemakaian klinik. Telah diketahui bahwa insufisiensi vertebrobasilaris tidak homogen dan terdiri dari berbagai kelainan pembuluh darah pada berbagai tempat dengan patogenesa serta prognosa klinis yang berbeda-beda. Oleh sebab itu, tidak mengherankan kalau dijumpai berbagai macam pendapat mengenai hasil pengobatan "end arterectomy" carotis pada kelompok penderita ini. Akhirnya, telah dibicarakan arti klinis penyakit atherosklerosis arteri innominata, subklavia dan vertebralis, serta hubungannya dengan sumbatan arteri carotis.

\section{Bab 5}

Manifestasi klinis dari daereh arteri vertebro-basilaris yang mengalami iskemi sementara umumnya lebih rumit daripada iskemi di daerah arteri carotis. Karena itu, pada bagian pertama bab ini, secara luas diterangkan simptomatologi serangan iskemi sementara ("Transient Ischemic Attacks") didaerah vertebrobasilaris. Arteri vertebralis segmen servikalis adalah satu-satu nya bagian pembuluh darah otak yang secara fungsionil berhubungan dengan perubahan degenerasi osteoartkritik kolumna servikalis. Karena itu, tangapan anatomis dengan patogenesa spondilosis kolumna servikalis serta maknanya dalam insufisiensi arteri vertebrobasilaris telah dibicarakan. Bagian 
terakhir bab ini berhubungan dengan patogenesa serta gambaran klinis dari sindroma "subclavian steal" .

\section{Bab 6}

Pembahasan singkat sejarah arteriografi kontras, khususnya arteriografi vertebralis. Kemudian dibahas komplikasi arteriografi sistim carotis maupun vertebralis secara terpilih, ketepatan arteriografi kontras cara konvensionil, serta angiografi substraksi "digital" intra vena. Bagian berikutnya dari bab ini mengenai tehnik diagnostik yang tidak invasif untuk mendiagnosa penyakit atherosklerotik sistem arteri innominata-subklavia-vertebralis. Telah dibahas tehnik "non imaging" serta "imaging Doppler" dan juga skan ultrasonik "real time"' bagian pembuluh darah tsb. Akhirnya telah dijelaskan prinsip dasar skan ultrasonik dupleks.

\section{Bab 7}

Bab ini membahas elemen dasar skan dupleks yang dihubungkan dengan peralatan peragaan dan perekaman. Selama penelitian telah digunakan 2 jenis "scan heads" yaitu jenis fokus menengah dan fokus pendek. Resolusi ruang kedua jenis skan telah dinilai dan telah dibahas pengaruh banyaknya volume tempat pengambilan contoh terhadap mutu sinyal Doppler dengan penafsiran bentuk gelombang spektral. Akhimya telah diikthisarkan tehnik modulasi-demolasi sinyal serta cara palaksanaannya.

\section{Bab 8}

Telaj diuraikan skan ultrasonik dupleks sistim carotis dan sistim arteri innominata-subklavia-vertebralis. Kemungkinan dan keterbatasan penyelidikan pada setiap bagian pembuluh darah otak telah dibahas. Bab inj juga menguraikan parameter Doppler secara kwantitatif yang digunakan pada keadaan tersumbatnya arteri innominata, subklavia dan vertebralis.

\section{Bab 9}

Bab ini menguraikan hasil penelitian kami mengenai skan ultrasonik dupleks arteri innominata-subklavia dan vertebralis sebagai tehnik yang tidak invasif dan hasilnya telah dibandingkan dengan arteriografi kontras. Pembahasannya dilakukan secara terpisah. Walaupun demikian sebelumnya telah diuraikan metoda yang dipakai dalam menentukan derajat penyempitan serta pengolahan data secara statistik. Dilakukan dua tahap penelitian skan ultrasonik dupleks sebagai dasar evaluasi data; tahap pertama berupa penelitian 
hanya pada arteri subklavia segmen proksimal berserta arteri vertebralis. Delam tahap kedua kami juga mengikutsertakan arteri innominata dan arteri vertebralis segmen servikalis. Ketepatan skan ultrasonik dupleks pada arteri innominata, subklavia dan ostium arteri vertebralis dinyatakan dalam nilai kepekaan dan ketepatan secara menyeluruh. Kebanyakan parameter menunjukkan bahwa skan ultrasonik dupleks pada segmen - segmen pembuluh darah otak tsb. dapat dipercayai dan dapat digunakan sebagai "screening test" penderita atherosklerosis otak. Hanya nilai kepekaan yang didapat dalam penentuan derajat penyempitan di daerah pangkal arteri subklavia dekstra terlalu rendah $(0.43)$ pada keadaan penyempitan diameter $\geq 50 \%$.

Juga diperlukan perbaikan dalam nilai ramalan positif dari skan ultrasonik dupleks ostium arteri vertebralis. Sebab yang mungkin didapatinya suatu hasil positif palsu ataupun yang negatif palsu telah diterangkan untuk setiap arteri secara terpisah. Umumnya keadaan aliran arteri vertebralis yang tinggi maupun rendah sangat mempersulit diagnosa andanya penyumbatan dari arteri ini. Ditambahkan pula, pada penelitian tahap kedua menunjukkan kemungkinan dapat dibedakannya keadaan hipoplasi dari sumbatan arteri vertebralis, apabila dilakukan oleh pemeriksa yang berpengalaman.

Penelitian dari ateri vertebralis segmen servikalis hanya dilakukan pada penderita dengan riwayat insufisiensi vertebrobasilaris atau apabila diminta oleh dokter. Khususnya pada bagian arteri vertebralis ini, telah dinilai hubungan antara taji osteo-artritik akibat spondilosis servikalis dengan pergeseran atau penyumbatan dari arteri vertebralis. Walaupun jumlah penderita dengan kelainan pada segmen servikalis sangat sedikit, hasil yang cukup terpercaya diperoleh dengan skan ultrasonik dupleks didaerah ini. Juga telah dinilai peranan skan ultrasonik dupleks pada keadaan penyumbatan hebat daerah segmen intradural dari arteri vertebralis. Interpretasi rekaman dari bentuk-bentuk sonagram pada sumbatan daerah intrakranial dari arteri vertebralis tampaknya lebih sulit dibandingkan dengan sumbatan daerah carotis. Hal ini mungkin karena lebih banyaknya variasi yang didapati pada daerah yang harus diperdarahi arteri vertebralis dengan penyempitan intrakranial. Akhirnya telah dibahas hasil dari skan ultrasonik dupleks arteri vertebralis pada penderita dengan sindroma "subclavian" atau "innominate steal". Utuk ini, jaga digunakan data angiografi substraksi "digital" intra vena.

\section{Bab 10}

Bab ini menguraikan pentingnya peranan angiografi substraksi "digital" intra vena dan gambaran ultrasonik dalam penyakit atherosclerosis, khususnya atherosclerosis arteri serebralis. Selanjutnya dibahas benturan - benturan yang didapat antar "ocular pneumoplethysmography", angiografi substraksi 
"digital" intra vena dan skan ultrasonik dupleks pada tata-laksana perawatan atherosklerosis otak dalam rumah sakit kami. Dengan dimanfaatkannya keiga cara diagnostik tsb. diatas sebagai inti "screening test" pada semua penderita, akan didapati hasil yang cukup terpercaya mengenai keadaan ekstra-kranial maupun intra-kranial dari arteri serebralis. Kebutuhan akan arteriografi kontras cara konvensionil pada atherosklerosis otak jelas menurum dalam dua tahun terakhir ini. Ditambahkan pembahasan mengenai kemungkinan serta keterbatasan pemakaian skan ultrasonik dupleks pada arteri innominata, subklavia dan vertebralis. Akhirnya diberikan beberapa saran dan harapan dimasa depan untuk pemeriksaan tidak invasif yang lengkap dan tepat untuk sistim arteri innominata-subklavia dan vertebralis.

\subsection{JAPANESE}

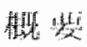

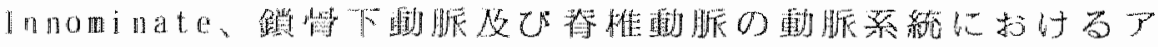

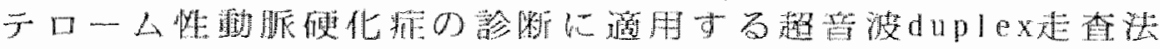

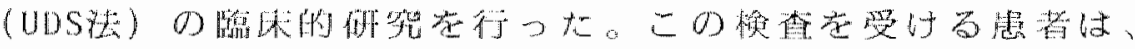

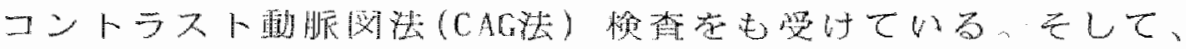

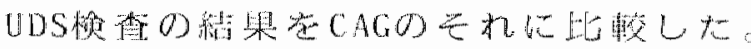

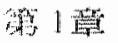

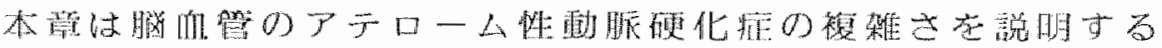

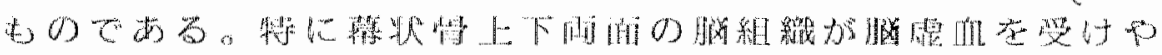

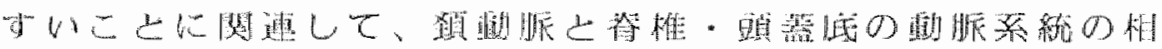

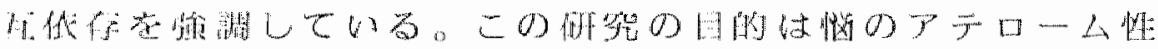

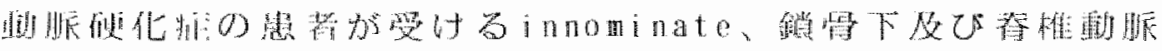

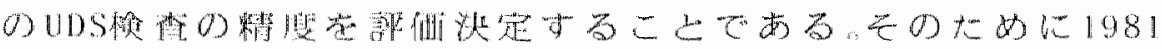

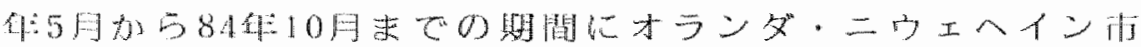

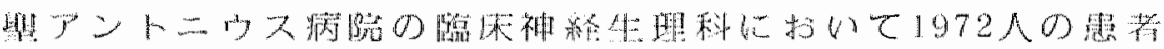

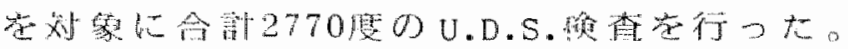


第2㱏

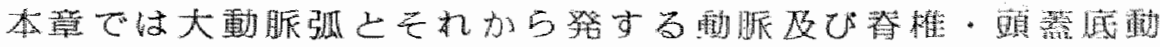

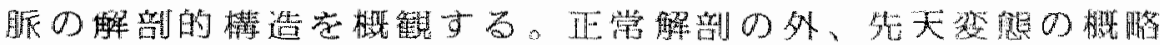

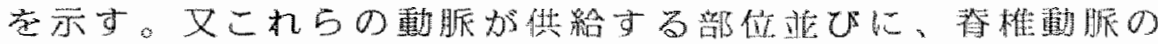

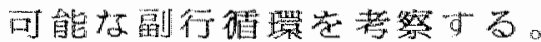

第3 3 章

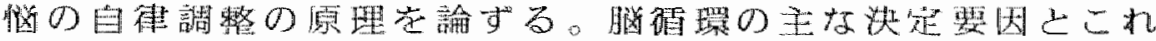

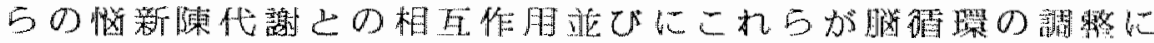

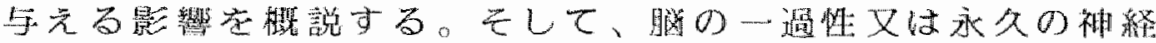

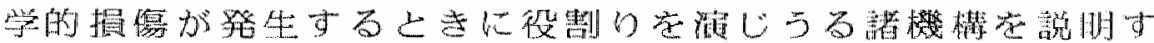
万。

第 4 笡

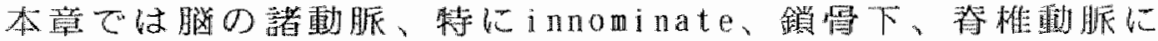

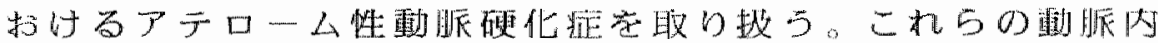

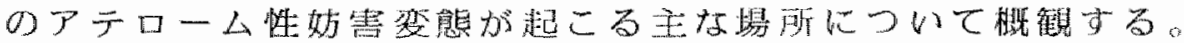

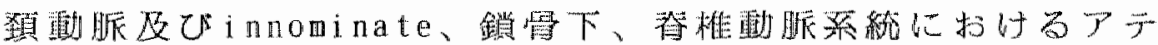

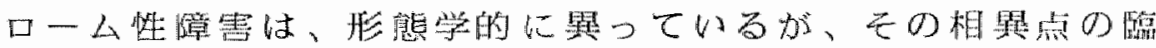

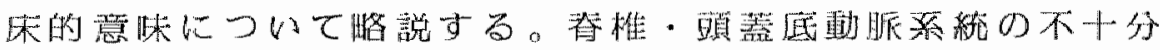

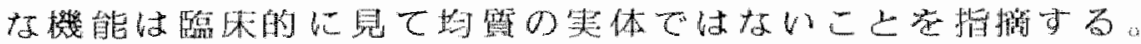

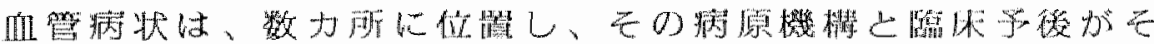

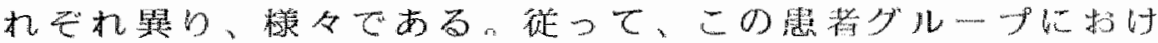

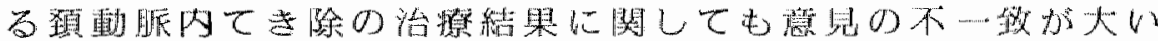
にあることは整くには当たらない。るして、innominate、隺

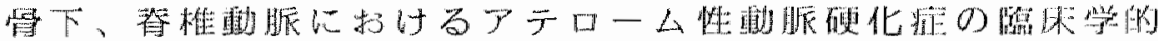

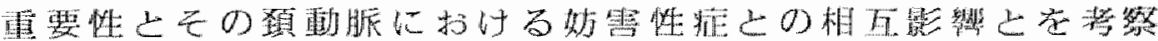
する。 
$5 x^{x}$

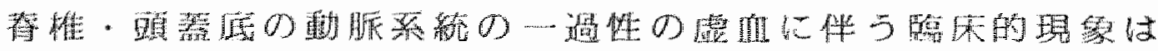

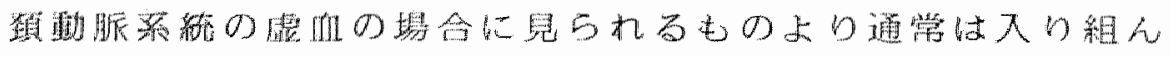

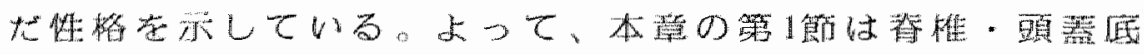

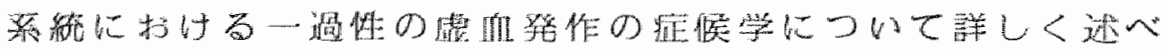

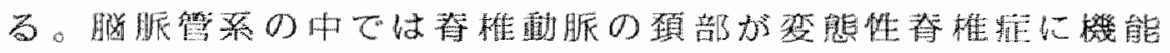

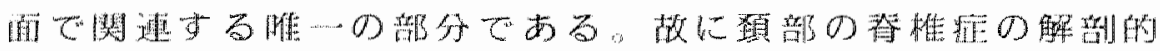

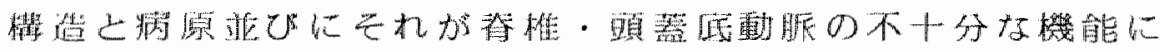

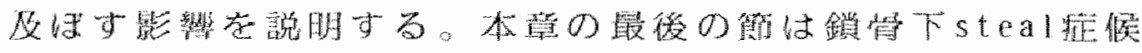

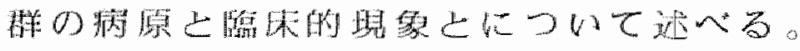

arit

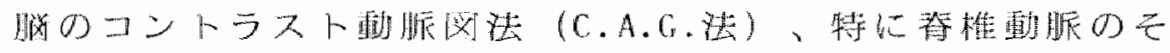

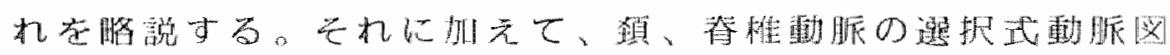

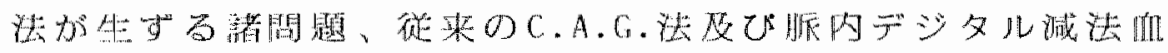

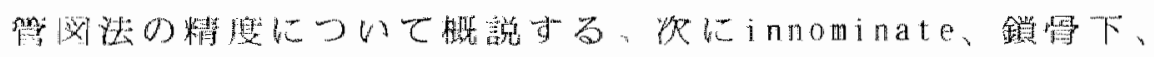

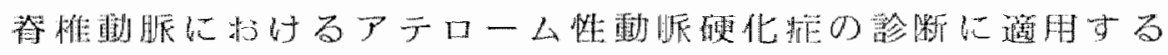

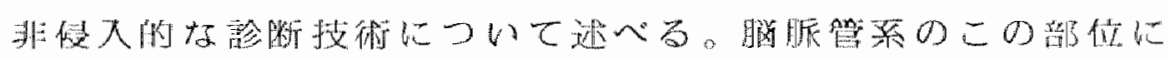

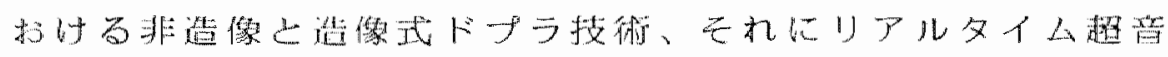

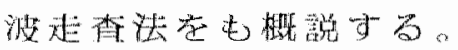

管 7 等

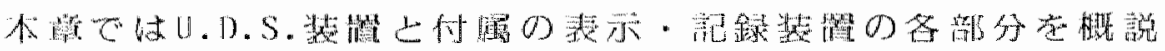

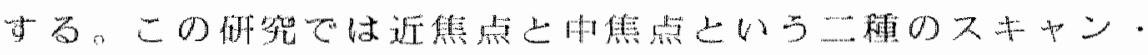

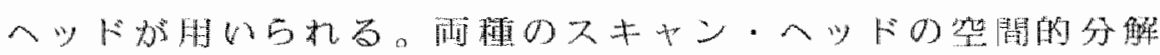

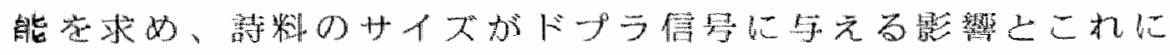

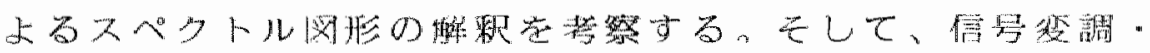

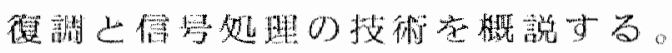




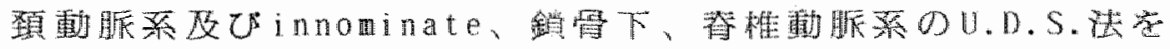

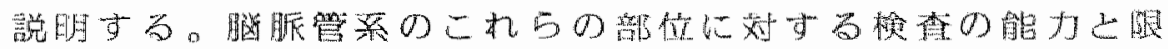

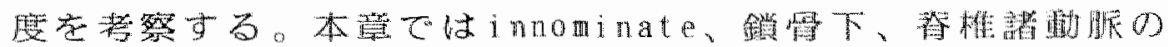

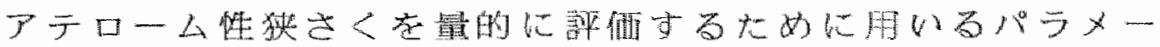
父一定定す。

\section{第 9 营}

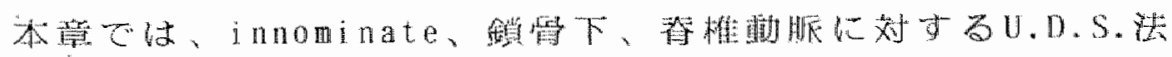

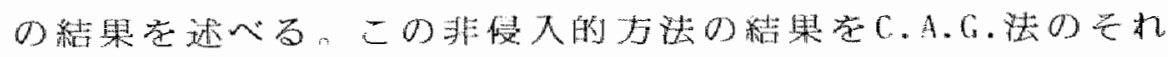

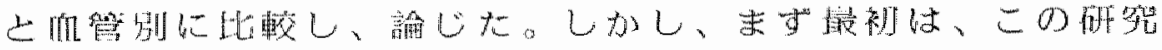

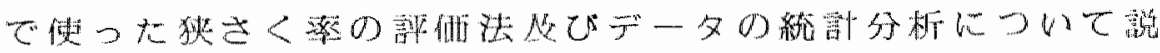
明している。

二つの研究がU.D.S.法のデータ詐相の基碳察なしている。は じかの研究では、销骨下、春椎闻動脈の基部に近い衫分だけ

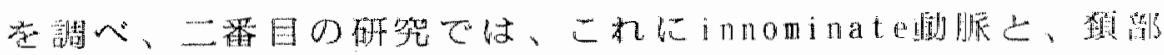
の篹椎動脈字加えて㒛べた。

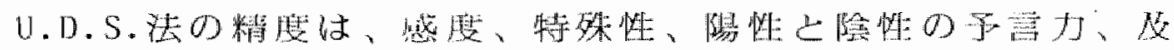

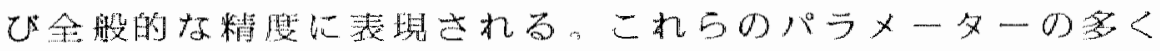

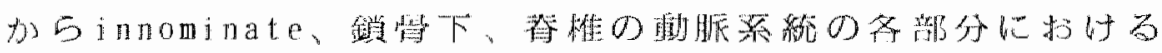

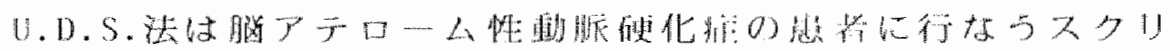

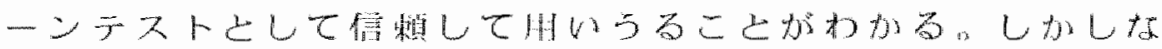

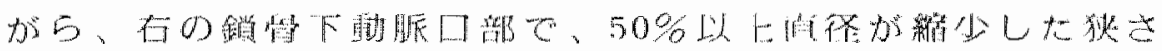

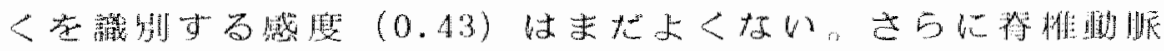

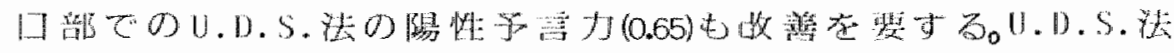

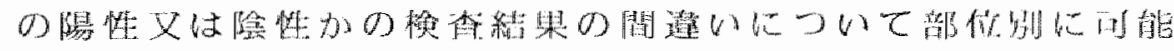

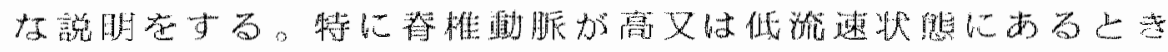

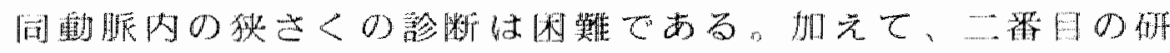

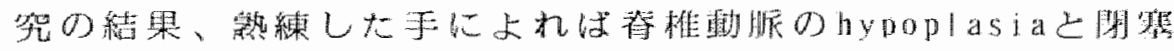




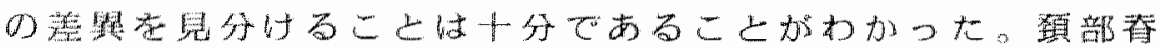

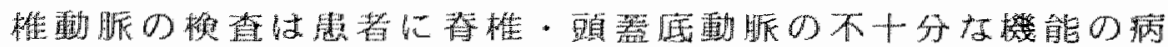

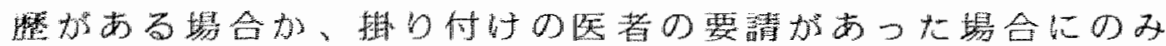
行垫っている。とりわけ替椎動脈のこの部分において、頚部

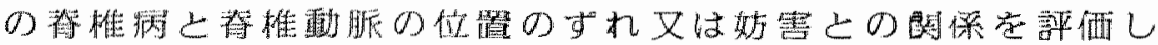

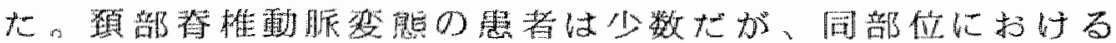
U.D.S.法が信頼しうる㛟榃なのは明白になった。春椎動脈の

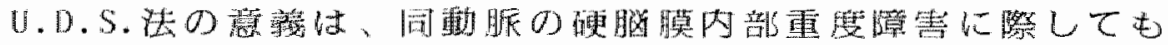

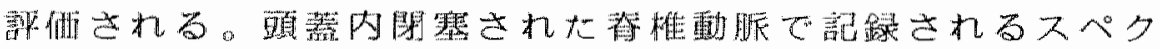

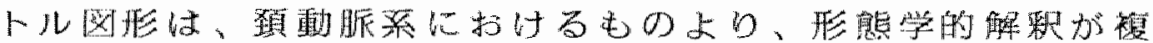

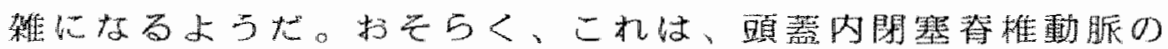

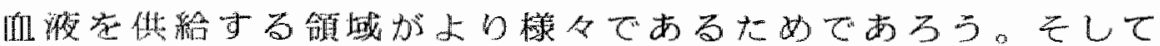

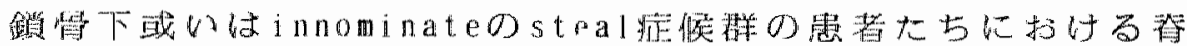

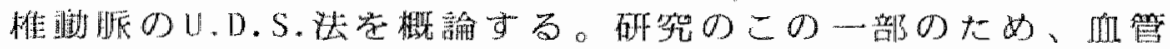
内デジタル瑊法血管図法のデータも使朋した。

第 10 福

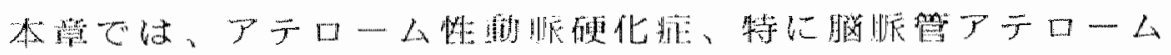

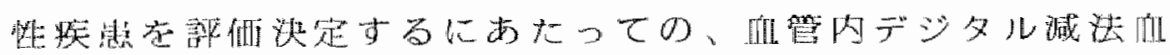

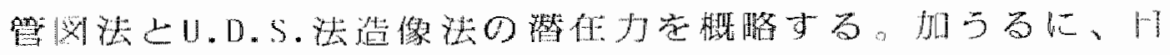

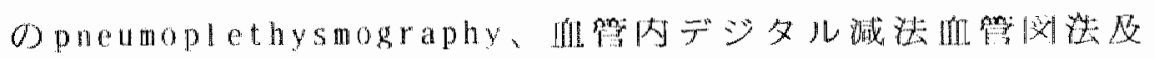

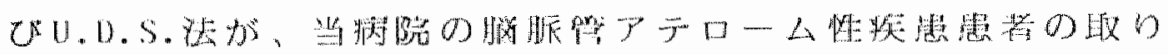

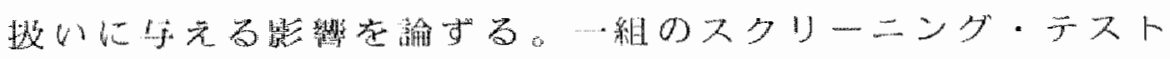

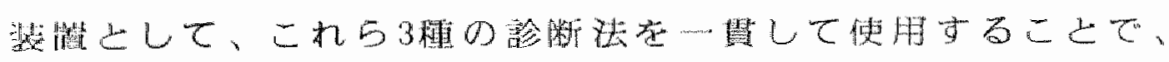

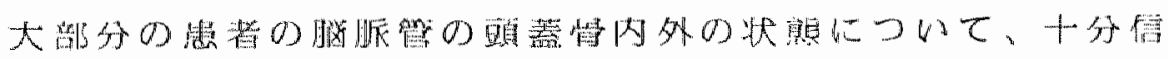

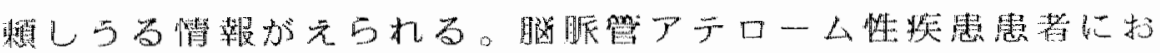
ける、愢例的存C.A.G.法の必要度は、過去2年にわたって、

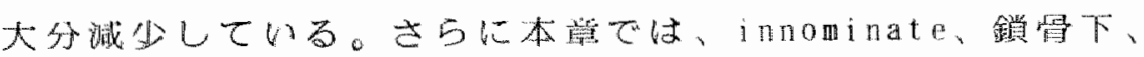
等椎動脈に対するU.D.S.法の可能性上险界を諭ずる。そして 


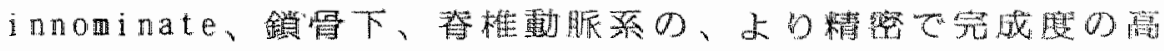

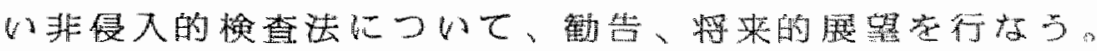




\section{REFERENCES}

ACKERSTAFF, R.G.A., EIKELBOOM, B.C., HOENEVELD, H., MOLL, F.L., VRIES, A.R. de.

The accuracy of ultrasonic duplex scanning in carotid artery disease.

Clinical Neurology and Neurosurgery, 84-4 (1982), 211-220.

ACKERSTAFF, R.G.A., HOENEVELD, H. ,SLOWIKOWSKI, J.M., MOLL, F.L., EIKELBOOM, B.C., LUDWIG, J.W.

Ultrasonic duplex scanning in atherosclerotic disease of the innominate, subclavian, and vertebral arteries. A comparative study with angiography.

Ultrasound in Medicine and Biology, 10 (1984), 409-418.

ALPERS, B.J., BERRY, R.G.

Circle of Willis in cerebral vascular disorders. The anatomical structure.

Archives ot Neurology, $8(1963), 398-402$.

BARBER, F.E.,BAKER, D.W. NATION, A.W.C., STRANDNESS, D.E. JR., REID, J.M.

Ultrasonic duplex echo-Doppler scanner.

IEEE Transactions on Biomedical Engineering, 2』 (1974), 109-113.

BARNES, R.W., WILSON, M.R.

Doppler ultrasound evaluation of cerebrovascular disease.

University of Iowa Press, Iowa, (1975).

BARNETT, H.J.M., JONES, M.W., BOUGHNER, D.R., KOSIUK, W.J.

Cerebral ischaemic events associated with prolapsing mitral valve.

Archives of Neurology, $33(1976), 777-782$.

BARNETT, H.J.M., BOLGHNER, D.R., TAYLOR, D.W., COOPER, P.E., KOSTUK, W. J., NICHOL, P.M.

Further evidence relating mitral-valve prolapse to cerebral ischemic events.

New England Iournal of Medicine, 302 (1980), 139-144.

BARNETT, H.J.M.

Embolism in mitral valve prolapse.

Annual Reviews of Medicine, 33 (1982), 489-507.

BARTON, J.W., MARGOLIS, M.T.

Rotational obstruction of the vertebral artery at the atlanto-axial joint.

Neuroradiology, $9(1975), 117 \times 120$.

BAUER, R., SHEEHAN, S., MEYER, J.S.

Arteriographic study of cerebrovascular disease.

2. Cerebral symptoms due to kinking, tortuosity, and compression of carotid and vertebral arteries in the neck.

Archives of Neurology, 4 (1961), 119-131.

BAYLISS, W.M.

On the local reactions of the arterial wall to changes of internal pressure.

Journal of Physiology, 28 (1902), 220. 
BERG, D. van den., DRIFT, J.H.A. van der.

Vertebro-basilaire angiografie via catheterisatie van de arteria brachialis.

Nederlands Tijdschrift woor Geneeskunde, 107 (1963), 1743-1748.

BERGUER, R., HIGGINS, R., NELSON, R.

Noninvasive diagnosis of reversal of vertebral-artery flow,

New England Journal of Medicine, 302 (1980), 1349-1351.

BERRY, R.G.

Discussion of "Collateral circulation of the brain" .

Neurology, 11 (1961), 20-22.

BHARADVAJ, B.K., MABON, R.F., GIDDINS, D.P.

a. Steady flow in a model of the human carotid bifurcation. Part I - flow visualization. Journal of Biomechanics, 15 (1982), 349-362.

b. Steady flow in a model of the human carotid bifurcation. Part II - Laser-Doppler anemometer measurements.

Journal of Biomechanics, 15 (1982), 363-378.

BLACKSHEAR, W.M., PHILLIPS, D.J., THIELE, B.L., HIRSCH, J.H., CHIKOS, P.M., MARINELLI, M.R., WARD, K.J., STRANDNESS, D.E. JR.

Detection of carotid occlusive disease by ultrasonic imaging and pulsed Doppler spec. trum analysis.

Surgery, $86(1979), 698-706$.

BLACKSHEAR, W.M. JR., PHILLIPS, D.J., CHIKOS, P.M., HARLEY, J.D., THIELE, B.L., STRANDNESS, D.E. JR.

Carotid artery velocity patterns in normal and stenotic vessels.

Stroke, $11(1980), 67-71$.

BLACKWOOD, W., HALLPIKE, J.F., KOCEN, R.S., MAIR, W.G.P.

Atheromatous disease of the carotid arterial system and embolism from the heart in cerebral infarction: A morbid anatomical sudy.

Brain, 92 (1969), 897-910.

BLAISDELL, W.F., CLAUSS, R.H., GALBRAITH, J.G., IMPARATO, A.M., WYLIE, E.J.

Joint study of extracranial arterial occlusion. IV. A review of surgical considerations. Journal of the American Medical Association, 209 (1969), 1889-1895.

BLANKENHORN, D.H., BROOKS, S.H., SELZER, R.H., BARNDT, R. JR. The rate of atherosclerosis change during treatment of hyperipoproteinemia.

Circulation, 57 (1978), 355-361.

BODILY, K.C., PHILLIPS, D.J., THIELE, B.L., STRANDNESS, D.E. JR. Noninvasive detection of internal carotid artery occlusion.

Angiology, 32 (1981), 517-521.

BOGOUSSLAVSKY, J, REGLI, F.

Delayed TIAs distal to bilateral occlusion of carotid arteries - Evidence for embolic and hemodynamic mechanisms.

Stroke, 14 (1983), 58-61. 
BOGOUSSLAVSKY, I., USKE, A., REGLI, F.

Carotid artery occlusion.

Delayed embolic ischemia for vertebrobasilar atheromatosis.

Archives of Neurology, $4 \mathbb{1}(1984), 334-335$.

BOGOUSSLAVSKY, $\mathbb{J}$, REGLI, F.

Vertebrobasilar transient ischemic attacks in intemal carotid artery occlusion or tight stenosis.

Archives of Neurology, $42(1985), 64-68$.

BOSNIAK, M.A.

An analysis of some anatomic-roentgenologic aspects of the brachiocephalic vessels.

American Journal of Roentgenology, $91(1964, a), 1222-11231$.

BOSNIAK, M.A.

Cervical arterial pathways associated with brachiocephalic occlusive disease.

American Journal of Roentgenology, 91 (1964,b), 1232-1244.

BRADSHAW, P., McQUIAD, P.

The syndrome of vertebro-basilar insufficiency.

Quarterly Journal of Medicine, 32, (1963), 279-296.

BRAIN, D.M.

Some unsolved problems of cervical spondylosis.

British Medical Journal, 23 (1963), $771-777$.

BRESLAU, P.J.

Ultrasonic duplex scanning in the evaluation of carotid artery disease.

Thesis, Schrijen-Lippertz B.V. Voerendaal, Heerlen, (1982).

BRESLAU, P.J., FELL, G., PHILLIPS, D.E., THIELE, B.L., STRANDNESS, D.E. $\mathbb{J R}$.

The role of common carotid artery velocity patterns in the evaluation of carotid bifurcation disease.

Archives of Surgery, 117 (1982), 58-60.

BROWN, B.ST.J., TATLOW, W.F.T.

Radiographic studies of the vertebral arteries in cadavers.

Effects of position and traction of the head.

Radiology, 81 (1963), 80-88.

BROWN, B.G., BOLSON, E., FRIMER, M., DODGE, H.T.

Quantitative coronary arteriography: estimation of dimensions, hemodynamic resistance, and atheroma mass of coronary artery lesions using the arteriogram and digital computation.

Circulation, 55 (1977), 329-337.

BRUINS SLOT, H., STR YBOSCH, L., GREEP, J.M.

Interobserver variability in single-plane aortography.

Surgery, $90(1981), 497-503$. 
CALA, L.A., ARMSTRONG, B.K.

A "Triple-steal syndrome" resulting from innominate and left subclavian arterial occlusion.

Australian and New Zealand Journal of Medicine, 3 (1972), 275-277.

CAPLAN, L.R. ROSENBAUM, A.E.

Role of cerebral angiography in vertebrobasilar occlusive disease.

Journal of Neurology, Neurosurgery and Psychiatry, 38 (1975), 601-612.

CAPLAN, L.R.

"Top of the basilar" syndrome.

Neurology, 30 (1980), $72-79$.

CAPLAN, L.R.

Vertebrobasilar disease. Time for a new strategy.

Stroke, 12 (1981), 111-114.

CAPLAN, L.R.

Bilateral distal vertebral artery occlusion.

Neurology, 33 (1983), 552-558.

CARTLIDGE, N.E.F., WHISANT, J.P., ELVEBACK, L.R.

Carotid and vertebral-basilar transient cerebral ischemic attacks. A community study, Rochester, Minnesota.

Mayo Clinic Proceedings, 52 (1977), 117-120.

CASTAIGNE, P., LHERMITTE, F., GAUTIER, J.C., ESCOUROLLE, R., DE ROUSNE, C., DER AGOPIAN, P., POPA, C.

Arterial occlusions in the vertebro-basilar system. A study of 44 patients with postmortem data.

Brain, 96 (1973), 133-154.

CHIKOS, P.M., FISHER, L.D., HIRSCH, J.H., HARLEY, J.D., THIELE, B.L., STRANDNESS, D.E. JR.

Observer variability in evaluating extracranial carotid artery stenosis.

Stroke, 14 (1983), 885-892.

CHILCOTE, W.A., MODIC, M.T., PAVLICEK, W.A., LITTLE, J.R., FURLAN, A.J., DUCHESNEAU, P.M., WEINSTEIN, M.A.

Digital subtraction angiography of the carotid arteries: a comprehensive study in 100 patients.

Radiology, $\mathbb{1} 39(1981), 287-295$.

CONSTANTIN, P., LUCRETIA, C.

Relations between the cervical spine and the vertebral arteries.

Acta Radiologica, $\mathbb{1}(1971), 91-96$.

CONTORNI, L.

Il circolo collaterale vertebro-vertebrale nella obliterazione dell'arteria succlavia alla sua origine.

Minerva Chirurgica, $15(1960), 268-271$. 
COOPERBERG, P.L., ROBERTSON, W.D.,FRY, P., SWEENEY, V.

High resolution real time ultrasound of the carotid bifurcation.

Journal of Clinical Ulirasound, 7 (1979), 13-17.

CORSON, J.D. MENZOIAN, J.O., LOGERFO, F.W.

Reversal of vertebral artery blood now demonstrated by Doppler ultrasound.

Archives of Surgery, $112(1977), 715-719$.

D"ALECY, L.G.

The cerebral circulation.

In: Physiology and biophysics, II Circulation, Respiration and fluid balance. ed.

Ruch, Th. C., Patton, H.D., W.B. Saunders Company, Philladelphia, (1974).

DASELER, E.H., ANSON, B.J.

Surgical anatomy of the subclavian artery and its branches.

Surgery, Gynaecology and Obstetrics, 108 (1959), 149-174.

DE BONO, D.P., WARLOW, C.P.

Potential sources of emboli in patients with presumed transient cerebral or retinal ischaemia.

Lancet, I (1981), 343-346.

DE BRAY, I-M., DAUZAT, M., TEISSEIRE-GIROD, F., DAVINROY, M., EMILE, J.

L'effet Doppler appliqué à l'étude des artères vertébrales. Resultats et corrélations artériographiques dans 25 cas.

La Nouvelle Presse Médicale, 7 (1978), 39-42.

DENNY-BROWN, D, MEYER, J.S.

The cerebrall collateral circulation.

2. Production of cerebral infarction by ischemic anoxia and its reversibility in early stages.

Neurology, $7(1957), 567-579$.

DRAKE, W.E., DRAKE, M.A.L.

Clinical and angiographic correlates of cerebrovascular insufficiency.

American Journal of Medicine, 45 (1968), 253-270.

EARNEST,F "HOUSER, O.W., FORBES, G.S., KISPERT, D.B., FOLGER, N., SUNDT, TH.M. JR.

The accuracy and limitations of intravenous digital subtraction angiography in the evalution of atherosclerotic cerebrovascular disease: angiographic and surgical corre* lation.

Mayo Clinic Proceedings, 58 (1983), 735-746.

EARNEST, F., FORBES, G., SANDOK, B.A., PIEPGRAS, D.G., FAUST, R.J., ILSTRUP, D.M., ARNDT, L.J.

Complications of cerebral angiography: prospective assessment of risk.

American Journal of Roentgenology, 142 (1984), 247-253. 
EASTCOTT, H.H.G., PICKERING, G.W. ROB, C.

Reconstruction of internal carotid artery in a patient with intermittent attacks of hemiplegia.

Lancet, III (1954), 994-996.

EDWARDS, J.H., KRICHEFF, I.I., RILES, TH., IMPARATO, A.M.

Angiographically undetected ulceration of the carotid bilurcation as a cause of embolic stroke.

Radiology, 132 (1979), 369-373.

EIKELBOOM, B.C.

Evaluation of carotid artery disease and potential collateral circulation by ocular pneumoplethysmography.

Thesis, Uitgeversmaatschappij Huisartsenpers B. V., Utrecht, (1981).

EIKELBOOM, B.C., ACKERSTAFF, R.G.A., LUDWIG, J.W., MOLL, F.L., VRIES, A.R. de, VERMEULEN, F.E.E.

Digital video subtraction angiography and duplex scanning in assessment of carotid artery disease. Comparison with conventional angiography.

Surgery, $94(1983), 821-825$.

EIKELBOOM, B.C., RILES, TH.R., MINTZER, R., BAUMANN, F.G., DEFILL.IP, G. , LIN, J., IMPARATO, A.M.

Inaccuracy of angiography in the diagnosis of carotid ulceration.

Stroke, $14(1983), 882-885$.

FARIS, A.A., POSER, C.M., WILMORE, D.W. , AGNEW, C.H.

Radiologic visualization of neck vessels in therlthy men.

Neurology, 13 (1963), 386-396.

FAUGHT, E., TRADER, S.D., HANNA, G.R.

Cerebral complications of angiography for transient ischemia and stroke: prediction of risk.

Neurology, 29(1979), 4-15.

FELL, G., PHILLIPS, D.J., CHIKOS, P.M., HARLEY, J.D., THIELE, B.L., STRANDNESS, DE. JR.

Ultrasonic duplex scanning for disease of the carotid artery.

Circulation, 64 (1981), 1191 1195.

FIELDING, J.W.

Cineroentgenogr aphy of the normal cervical spine.

Journal of Bone and Joint Surgery, 39.A (1957), 1280-1288.

FUELDS, W.S.

Reflections of "The subclavian steal".

Stroke, 1. (1970), 320-324.

FIELDS, W.S., MASLENIKOV, C., MEYER, I.S.

Joint study of extracranial arterial occlusion: V. Progress report of prognosis following surgical or nonsurgical treatment for transient cerebral ischaemic attacks and cervical carotid artery lesions.

Journal of the American Medical Association, 211 (1970), 1933-2003. 
FIELDS, W.S. LEMAK, N.A.

Joint study of extracranial arterial occlusion.

VII. Subclavian steal - A review of 168 cases.

Journal of the American Medical Association, 222 (1972), 1139.1143.

FISHER, C.M.

Occlusion of the internal carotid artery.

Archives of Neurology and Psychiatry, 65 (1951), 346-377.

FISHER, C.M.

Observations of the fundus oculi in transient monocular blindmess.

Neurology, 9 (1959), 333-347.

FISHER, C.M.

A new vascular syndrome - 'The subclavian steal".

New England Journal of Medicine, 265 (1961), 912-913.

FISHER, C.M., KARNES, W.E., KUBIK, C.S.

Lateral medullary infarction: the pattern of valscular occlusion.

Journal of Neuropathology and Experimental Neurology, 20 (1961), 323-379.

FISHER, C.M., GORE, I. OKABE, N., WHITE, P.D.

Atherosclerosis of the carotid and vertebral arteries - extracranial and intracranial.

Journal of Neuropathology and Experimental Neurology „ 24 (1965), 455-476.

FISHER, C.M.

Lacunes: small deep cerebral infarcts.

Neurology, $15(1965), 774-784$.

FISHER, C.M.

A lacunar stroke. The dysarthria - clumsy hand syndrome.

Neurology, 17 (1967), 614-617.

FISHER, C.M.

Occlusion of the vertebral arteries causing transient basilar symptoms.

Archives of Neurology, 22 (1970), 13-19.

FISHER, C.M., CAPLAN, L..R.

Basilar artery branch occlusion: a cause of pontine infarction.

Neurology, 21 (1971), 900-905.

FOLCARELLI, P., EIKELBOOM, B.C., RILES, TH.S., IMPARATO, A.M.

Pitfalls of carotid artery surgery based on digital subtraction angiography.

Bruit, $7(1983), 13-17$.

FORD, J.J.JR., BAKER, W.H., EHRENHAFT, J.L.

Carotid endarterectomy for nonhemisferic transient ischemic attacks.

Archives of Surgery, $110(1975), 1314-1317$.

FRANCESCHI, C., VADROT, M., LUIZY, F., JARDIN, M., RIBADEAU-DUMAS, C. FRANCO, G., BOUZAC, $\mathrm{H}$.

Echotomografic anatomy of the supra aortic arterial trunks.

Ulirasons, 2 (1981), 57.61. 
GATZWEILER, W.

Die Spondylchondrose der Halswirbelsäule.

Zeitschrift für Rheumaforschung, 14 (1955), 368-371.

GAUTIER, J.C., MORELOT, D.

Infarctus cérëbraux.

La nouvelle presse médicale, 4 (1975), 2575-2580.

GILLIAN, L.A.

The correlation of the blood supply to the human brain stem with clinical brain stem lesions.

Journal of Neuropathology and Experimental Neurology, 23 (1964), 78-108.

GIROUX, J.C.

Vertebral artery compression by osteophytes.

Advances in Oto-Rhino-Laryngology, 28 (1982), 111-117.

GUNNING, A.J., PICKERING, G.W., ROBB-SMITH, A.H.T., ROSS RUSSEL, R.

Mural thrombosis of the internal carotid artery and subsequent embolism.

Quarterly Journal of Medicine, 33 (1964), 155-195.

HAFERKAMP, G., REGLI, F.

Das Krankheitsbild des doppelseitigen Carotisverschlusses.

Zeitschrift für Neurologie, 206 (1974), 103-115.

HANSON, M.R., CONOMY, J.P., HODGMAN, I.R.

Brain events associated with mitral valve prolapse.

Stroke, 11 (1980), 499-506.

HARDIN, C.A., WILLIAMSON, W.P., STEEGMAN, A.TH.

Vertebral artery insufficiency produced by cervical osteoarthritic spurs.

Neurology, $10(1960), 855-858$.

HARDIN, C.A.

Cerebral ischaemia due to extracranial vertebral artery occlusion.

Surgery, 52 (1962), 627-631.

HARRISON, M.J.G., MARSHALL, J.

The finding of thrombus at carotid endarterectomy and its relationship to the timing of surgery.

British Journal of Surgery, 64 (1977), $511-512$.

HASS, W.K., FIELDS, W.S., NORTH, R.R., KRICHEFF, I.I., CHASE, N.E., BAUER, R.B.

Joint study of extracranial arterial occlusion. II. Arteriography, techniques, sites, and complications.

Journal of the American Medical Associaton, 203 (1968), 961-968.

HAUGE, T.

Catheter vertebral angiography.

Acta Radiologica, suppl. 109, (1954). 
HEDA, K.,TOOLE, I.F., MCHENRY, L.C.

Carotid and vertebrobastlar iransient ischaemic attacks: clinical and angiographic correlation.

Neurology, 29(1979), 1094-1101.

HENNERICI, M., AULICH, A., SANDMANN, W.

Der Wert der Dopplersonographie für Prognose und Therapie schlaganfallgefärdeter Patienten.

Angio, $3(1980), 151-160$.

HENNERICI, M., AULJCH, A., SANDMANN, W. FREUND, H-J.

Incidence of asymptomatic extracranial arterial disease.

Stroke, $12(1981), 750-758$.

HENNERICI, M., RAUTENBERG, W., MOHR, S.

Stroke risk from symptomless extracranial arterial disease.

Lancet, II (1982), $1180=1183$.

HENNERICl, M., FREUND, H-J.

Efficacy of CW-Doppler and duplex system examinations for the evaluation of extracranial carotid disease.

Journal of Clinical Ultrasound, 12 (1984), 155-161.

HESSEL, S.J.,ADAMS, D.F., ABRAMS, H.L.

Complications of angiography.

Radiology, 138 (1981), 273-281.

HESSELINK, J.R, TERESI, L.M., DAVIS, K.R., TAVERAS, J.M.

Intravenous digital subtraction angiography of arteriosclerotic vertebrobasilar disease.

American Journal of Roentgenology, 142 (1984), 255-260.

HEYMAN, A., WILKINSON, W.E., HURWITZ, B.J., HAYNES, C.S., UTLEY, C.M.

Clinical and epidemiologic aspects of vertebrobasilar and nonfocal cerebral ischemia.

In: Vertebrobasilar arterial occlusive disease. ed. Berguer, R., Bauer, R.B., Raven Press, New Yo:k, (1.984), 27-36.

HOEKS, A.P.G., RUISSEN, C.J., HICK, P., RENEMAN, R.S.

Methods to evaluate the sample volume of pulsed Doppler systems.

Ultrasound in Medicine and Biology, 10 (1984), 427-434.

HOFFMAN, M.G., GOMES, A.S., PAIS, S.O.

Limitations in the interpretation of intravenous carotid digital subtraction angiography.

American Journal of Roentgenology, 142(1984), 261-264.

HOWIESON, J. MEGISON, L.C. JR.

Complications of vertebrall artery catheterization.

Radiology, 91 (1968), 1109-1111.

HOWLAND, W.J., CURRY, J.L.

Transient cerebral blindness - A hazard of vertebral artery catheterization.

Radiology, 83 (1964), 428-432. 
HOYT, W.F.

Transient bilateral blurring of vision. Considerations of a episodic ischemic symptom of vertebral-basilar insufficiency.

Archives of Ophthalmology, $70(1963), 746-751$.

HÜLSE, M.

Differentialdiagnose der Schwindelbeschwerden bei funktionellen Kopfgelengksstörungen und bei vertebrobasilärer Insuffizienz.

H.N.O, $30(1982), 440-446$.

HULT, L.

Cervical, dorsal and lumbar spinal syndromes.

Acta Orthopaedica Scandinavica, supplement 17 (1954), 1-102.

HUMPHRIES, A.W., YONG, J.R., BEVEN, E.G., LE FEVRE, F.A., WOLFE, V.G. de.

Relief of vertebrobasilar symptoms by carotidendarterectomy.

Surgery, 57 (1965), 48-52.

HUTCHINSON, E.C., YATES, P.O.

The cervical portion of the vertebral artery. A clinico-patholigical study.

Brain, 79 (1956), 319-331.

HUTCHINSON, E.C., YATES, P.O.

Carotico-vertebral stenosis.

Lancet, I (1957), 2-8.

IMPARATO, A.M., RILES, TH. S., GORSTEIN, F.

The carotid bifurcation plaque: Pathologic findings associated with cerebral ischemia. Stroke, 10 (1979), 238-245.

IMPARATO, A.M., RILES. TH.S., KIM, G-E.

Cervical vertebral angioplasty for brain stem ischemia.

Surgery, $90(1981), 842-852$.

JAMES, E.M., EARNEST, F., FORBES, G.S., REESE, D.F., HOUSER, O.W., FOLGER, W.N.

High-resolution dynamic ultrasound imaging of the carotid bifurcation: a prospective evaluation.

Radiology, 144 (1982), 853-858.

JOFE, M.H., WHITE, A.A., PANJABI, M.M.

Physiology and Biomechanics.

In: The cervical spine, ed. Bailey, R.W., J.B. Lippencott company, Philadelphia, $(1983), 24-35$.

JONES, H.R., MILLIKAN, C.H., SANDOK, B.A.

Temporal profile (clinical course) of acute vertebrobasilar system cerebral infarction.

Stroke, 11(1980), 173-177. 
JORGENSEN, L., TOR VIK A.

Ischaemic cerebrovascular disease in an autopsy series. Part. 1. Prevalence, location and predisposing factors in verified thrombo-embolic acclusions, and their significance in the pathogenesis of cerebral infarction.

Journal of the Neurological Sciences, 3 (1966), 490-509.

KANEDA. H., IRINO, T., MINAMI, T., TANEDA, M.

Diagnostic reliability of the percutaneous ultrasonic Doppler techniques for vertebral arterial occlusive disease.

Stroke, 8(1977), 572-559

KATZ, M.L., JOHNSON, M., POMAJZL, M.J., COMEROTA, A.J., AHRENSFIELD, D., MANDEL, L., HAYDEN, W., FOGARTY, TH.

The senstitivity of real-time B-mode carotid imaging in the detection of ulcerated plaques.

Bruit, 8 (1983), 13-17

KELLER, H.M., MEIER, W.E., KUMPE, D.A.

Noninvasive angiography for the diagnosis of vertebral artery disease using Doppler ultrasound (Vertebral artery Doppler).

Stroke, 7 (1976), 364-369.

KENDELL, R.E., MARSHALL, J.

Role of hypotension in the genesis of transient focal cerebral ischaemic attacks.

British Medical Journal, II (1963), 344-348.

KISHORE, P.R.S., CHASE, N.E., KRICHEFF, I.I.

Carotid stenosis and intracranial emboli.

Neuroradiology, 100 (1971), 351-356.

KLEYN, A. de, VERSTEEGH, C.

Über verschiedene Formen von Ménières Syndrom.

Deutsche Zeitschrift für Nervenheilkunde, 132 (1933), 157-189.

KNOX, R.A., PHILLIPS, D.J., BRESLAU, P.J., LAWRENCE, R., PRIMOZICH, J.F., STRANDNESS, D.E. JR.

Emperical findings relating sample volume size to diagnostic accuracy in pulsed Doppler cerebrovascular studies.

Journal of Clinical Ultrasound, 10 (1982-a), 227-232.

KNOX, R.A., BRESLAU, P.J. STRANDNESS, D.E. JR.

A simple parameter for accurate detection of severe carotid disease.

British Journal of Surgery, 69 (1982-b), 230-233.

KRAYENBÜHL, H., YASARGIL, M.G.

Cerebral angiography.

George Thieme Verlag, Stuttgart, 1982.

KROGDAHL, T., TORGERSEN, $O$.

Die Unco-Vertebralgelenke und die Arthrosis deformans Unco-Vertebralis.

Eine pathologisch-anatomische und röntgenologische Studie.

Acta Radiologica, 21 (1940), 231-262. 
KRUEGER, B.R., OKAZAKI, H.

Vertebral-basilar distribution infarction following chiropractic cervical manipula. tions.

Mayo Clinic Proceedings, 55 (1980), 322-332.

KUBALA, M.J., MILLIKAN, C.H.

Diagnosis, pathogenesis, and treatment of 'drop attacks'.

Archives of Neurology, 11 (1964), 107-113.

KUBIK, C.S., ADAML, R.D.

Occlusion of the basilar artery - A clinical and pathological study.

Brain, 69 (1946), 6-121.

LANGLOIS, Y., ROEDERER, G.O., CHAN, A., PHILLIPS, D.J., BEACH, K.W., MARTIN, D., CHIKOS, P.M., STRANDNESS, D.E. JR..

Evaluating carotid artery disease.

The concordance between pulsed Doppler/spectrum analysis and angliography.

Ultrasound in Medicine and Biology, 9 (1983), 51-63.

LASSEN, N.A., INGVAR, D.H., SKINHOJ, E.

Brain function and blood flow.

Scientific American, 239 (1978), 50-59.

\section{LAZORTHES, G.}

Pathology, classification and clinical aspects of vascular diseases of the spinal cord.

In: Handbook of Clinical Neurology, Vinken, P.J. Bruyn, G.W. Ed., vol. 12, NorthHolland Publishing Company, Amsterdam (1972), 492-506.

LIEBMAN, P.R., BARNES, R.W.

Internal carotid occlusion, hemispheric collateral perfusion pressure and stroke.

Stroke, $12(1981), 126$.

LOGERFO, F.W., CRAWSHAW, H.M., NOVAK, M., SERRALLACH, E., QUIST, W.C., VALERI, R.

Effect of flow split on separation and stagnation in a model vascular bifurcation. Stroke, $\rrbracket 2(1981), 660-665$.

LUSBY, R.J., EHRENFELD, W.K.

Carotid artery surgery based on digital subraction angiography.

American Journal of Surgery, 144 (1982), 211-214.

LUDWIG, J.W. GLAUDEMANS, P.W., ENGELS, P.H.C., VERMEULEN, F.E.E., EIKELBOOM, B.C.

Cinematographic examinations of lesions of the ostiae and first parts of the vertebral arteries.

In: Angiology, Balas, P. Ed., Plenum Press, New York, (1984), 49 \-495.

MANI, R.L., EISENBERG, R.I. MCDONALD, E.J. JR., POLLOCK, J.A., MANI, J.R.

Complications of catheter cerebral arteriography: Analysis of 5,000 procedures. I. Criteria and incidence.

American Journal of Roentgenology, 131 (1978,a), 861-865. 
MANIR.L., EISENBERG, R.L.

Complications of catheter cerebral arteriography: Analysis of 5,000 procedures. IIL. Assessment of arteries injected contrast medium used, duration of procedure, and age of patient.

American Journal of Roentgenology, $13 \Perp(1978, b), 871-874$.

MARSHALL, J.

The natural history of transient ischaemic cerebrovascular attacks.

Quarterly Journal of Medicine, 33 (1964), 309-324.

MCHENRY, L, C. $\mathbb{M R}$.

Cerebral blood flow and metabolism.

In: Cercbral vascular disease. ed. Harrison, M.J.G., Dyken, M.L., Butterworths, London, (1983).

MCNAMARA, J.O., HEYMAN, A., SILVER, D., MANDEL, M.E.

The value of carotid endarterectomy in treating transient cerebral ischaemia of the posterior circulation.

Neurology, 27 (1977), 682-684.

MERODE, T. van, HICK, P., HOEKS, A.P.G., RENEMAN, R.S.

Limitations of Doppler spectral broadening in the early detection of carotid artery disease due to the size of the sample volume.

Ultrasound in Medicine and Biology, 9(1983), 581-586.

MEYER, IJ.S., SHEEHAN, S. BAUER, R.B.

An arteriographic study of cerebrovascular disease in man.

1. Stenosis and occlusion of the vertebral-basilar arterial system.

Archives of Neurolgy, 2 (1960), 27-45.

MILLIKAN, C.H., SIEKERT, R.G.

Studies in cerebrovascular disease. I. The syndrome of intermittent insufficiency of the basilar arterial system.

Proceedings of the staff meetings of the Mayo Clinic, 30 (1955), 61-68.

MISTRETTA, C.A., ORT, M.G., CAMERON, I.R., CRUMMY, A.B., MORAN, P.R.

A multiple image subraction technique for enhancing low contrast, periodic objects.

Investigative Radiology, $8(1973), 43-49$.

MOL, J.M.F.A.

Doppler-laematotachografisch onderzoek bij cerebrale circulatiestoornissen.

Thesis, Pecassemeurozet, Maastricht, (1973).

MOL, J.M.F.A.

The clinical use of Doppler hematographic investigation in cerebral circulation disturbances.

In: Doppler ultrasound in the diagnosis of cerebrovascular disease, ed. Reneman, R.S., Hoeks, A.P.G., Research Studies Press, John Wiley, Chichester, (1982), 129 $\mathbb{1 5 6 .}$ 
MOLL, F.L.

Carotid bruits in asymptomatic patients - a prospective follow-up study.

Thesis, Elinkwijk B.V., Utrecht (1984).

MONIZ, E.

L'encéphalographie artérielle, son importance dans la localisation des tumeurs cérébrales.

Revue Neurologique, $34(1927), 72-89$.

MONIZ, E. ALVES, A.

L'importance diagnostique de l"artériographie de la fosse postérieure.

Revue Neurologique, (1933), 91-96.

MOOSSY, J.

Morphology, sites and epidemiology of cerebral atherosclerosis.

In: Cerebro vascular disease, vol. 51 : Proceedings of the Association for Research in nerwous and Mental Diseases, Williams and Wilkens, Baltimore, Maryland, 1-22 (1966).

MOUFARRY, N.A., LITTLE, J.R., FURLAN, A.J., WILLIAMS, G., MARZEWSKI, D.J.

Vertebral artery stenosis: Long-term follow-up.

Stroke, 15 (1984), 260-263.

NARITOMI, H., SAKAI, F., MEYER, J.S.

Pathogenesis of transient ischemic attacks within the vertebrobasilar arterial system. Archives of Neurology, 36 (1979), 121-128.

NEWTON, TH. H., POTTS, D.G.

Radiology of the skull and brain. Angiography, C.V. Mosby Company, Saint Louis, 1974.

\section{OLESON, $\mathrm{J}$.}

Contrallateral focal increase of cerebral blood-flow in man during arm work.

Brain, 94 (1971), 635-646.

OLIVECRONA, H.

Complications of cerebral angiography.

Neuroradiology, 14 (1977), 175-181.

OLSSON, O.

Vertebral angiography.

Acta Radiologica, 40 (1953), 103-107.

OUCHI, H., OHARA, I.

Extracranial abnormalities of the vertebral artery detected by selective arteriography. Journal of Cardiovascular Surgery, 18 (1973), 250-261.

OWENS, M.L., ATKINSON, J.B., WILSON, S.E.

Recurrent transient ischemic attacks after carotid endarterectomy.

Archives of Surgery, $115(1980), 482-486$. 
PAAL $G$.

Die intermithierende vertebrobasilare Insuffizienz.

Internist, 22 (1981), 327-339.

PARROTT, J.C.

The subclavian steal syndrome.

Archives of Surgery, 88 (1964), 661-665.

PATRICK, B.K., RAMIREZ-LASSEPAS, M., SNYDER, B.D.

Temporal profile of vertebrobasilar territory infarction.

Prognostic implications.

Stroke, $11(1980), 643-648$.

PATTERSON, H. JR.

Clinical diagnosis of arterial insufficiency in the vertebrobasilar system.

In: Clinical Neurosurgery, vol. 29, Williams and Wilkens, Baltimore, (1982), 482-488.

PAYNE, E.E., SPILLANE, J.D.

The cerwical spine. An anatomico-pathological study of 70 specimens (using a special technique) with particular reference to the problem of cerivical spondylosis.

Brain, 80 (1957), 571-596.

PEETERS, F.L.M.

Het vertebralisangiogram bij intracraniële tumoren.

Thesis, Centrale Drukkerij N.V., Nijmegen, (1969).

PFEIFFER, R.B. JR., GREGORY, R.T., SYNDER, S.O., JR., GAYLE, R.G., WHEEUER, J.R.

Non-invasive diagnosis of vertebral artery occlusive disease.

Bruit, 8 (1984), 164-167.

PHILLIPS, D.J., POWERS, J.E., EYER, M.K., BLACKSHEAR, W.M. JR., BODILY, K.C., STRANDNESS, D.E. JR., BAKER, D.W.

Detection of peripheral vascular disease using the duplex scanner III.

Ultrasound in Medicine and Biology, 6 (1980), 205-218.

POURCELOT, L.

Indications de l'ultrasonographie Doppler dans l'étude des vaisseaux périphériques.

La Revue du Praticien, 25, (1975), 4671-4680.

POURCELOT, I.. "RIBADEAU-DUMAS, J.L., FAGRET, D , PLANIOL, TH.

Apport' de l'examen Doppler dans le diagnostic du vol sous-clavier.

Revue Neurologique, 133 (1975), 309-323.

POURCELOT, L.

Contintous wave Doppler techniques in cerebral vascular disturbances.

In: Doppler ultrasound in the djagnosis of cerebrovascular disease, ed. Reneman,

R.S., Hoeks, A.P.G., Research Studies Press, John Wiley, Chichester, (1982)., 1.03128.

RADNER, $S$.

Vertebral angiography by cathetrization. A new method employed in 221 cases.

Acta Radiologica, suppl. 87. (1951). 
RAMIREZ-BOETTNER, C.M., SOSA, O.D., BALANZA, J., CALCENA, R.M.

'Subclavian steal syndrome' with reversal of blood flow in right carotid artery.

Annals of Internal Medicine, $64,(1966), 142-144$.

REED, R.L., SIEKERT, R.G., MERIDETH, J.

Rarity of transient focal cerebral ischaemia in cardiac dysrythmia.

Journal of the American Medical Association, 223 (1973), 893-895.

REILLY, L.M., LUSBY, R.J., HUGHES, L., FERRELL, L.D., STONEY, R.J., EHRENFELD, W.K.

Carotid plaque histology using real-time ultrasonography.

Clinical and therapeutic implications.

American Journal of Surgery, 146 (1983), 188-193.

REIVICH, M., HOLLING, H.E., ROBERTS, B., TOOLE, J.F.

Reversal of blood flow through the vertebral artery and its effect on cranial circulation.

New England Journal of Medicine, 265 (1961), 878-885.

RENEMAN, R.S., SPENCER, M.P.

Local Doppler audio spectra in normal and stenosed carotid arteries in man.

Ultrasound in Medicine and Biology, 5 (1979), 1-11.

REUTERN, G-M. von, BÜDINGEN, H.J., FREUND, H-J.

Dopplersonographische Diagnostik von Stenosen und Verschlüssen der Vertebralarterien und des Subclavian-Steal-Syndromes.

Archives für Psychiatrie und Nervenkrankheiten, 222 (1976), 209-222.

REUTERN, G-M. von, POURCELOT , $L$.

Cardiac cycle-dependent alternating flow in vertebral arteries with subclavian artery stenoses.

Stroke, $9(1978), 229-236$.

RILEY, L., FRIEDMAN, J.M.

Stroke, orthostatic hypotension, and focal seizures.

Journal of the American Medical Association, 245 (1981), 1243-1244.

RINGELSTEIN, E.B.

Neue Anwendugsmöglichkeiten der Dopplersonographie am hinteren Hirnkreislaul bei degenerativer Gefässkrankheit, angiotherapeutischen Eingriffen und epidemiologischen Untersuchungen.

Habilitationsschrift, Aachen, (1984).

RINGELSTEIN, E.B., KOLMANN, H.L., ZEUMER, H.

Carotis B-scan: Konkurrenz oder Ergånzung der Ultraschall-Dopplersonographie?

Deutsche Medizinische Wochenschrift, 107 (1982), 928-933.

RINGELSTENN, E.B., ZEUMER, H., HÜNDGEN, R., MEYA, U.

Angiologische und prognostische Beurteilung von Hirnstaminsulten.

Klinische, dopplersonographische und neuroradiologische Biefunde.

Deutsche Medizinische Wochenschrift, 108 (1983), 1625-1631. 
ROBERTSON, J.T.

Neck manipulation as a cause of stroke.

Stroke, $12(1981), 1$.

ROSENTHAL, D., COSSMANN, D. , LEDIG, B., CALLOW, A.D.

Results of carotid endarterectomy for vertebrobasilar insufficiency.

An evaluation of ten years.

Archives of Surgery, 113 (1978), 1361-1364.

ROSS RUSSELL, R.W.

Observations on the retinal blood vessels in monocular blindness.

Lancet, II (1961), 1.422-1.428.

ROSS RUSSELL, R.W.

Transient cerebral ischaemia.

In: Vascular disease of the central nervous system, ed.

Ross Russell, R.W., Churchitl Livingstone, Edingburgh (1983).

ROY, C.S., SHERRINGTON, C.S.

On the regulation of the blood-supply of the brain.

Journal of Physiology, II (1980), 85.

RUFF, R.L., TALMAN, W.T., PETITO, F.

Transient ischemic attacks associated with hypotension in hypertensive patients with carotid artery stenosis.

Stroke, $12(1981), 353-355$.

RUISSEN, C. J .

Frequentie-multiplexing bij medisch onderzoek.

Polytechnisch Tijdschrift, 35 (1980), 372-373.

SANTSCHI, D.R., FRAHM, C.J., PASCALE, L.R., DUMANIAN, A.V.

The subclavian steal syndrome. Clinical and angiographic considerations in 74 cases in adults.

Journal of Thoracic and Cardiovascular Surgery, 51 (1966), 103-112.

SCATLIFF, I.H., MISHKIN, M.M. HYDE, I.

Vertebral arteriography: An evaluation of methods.

Radiology, 85 (1965), 14-21.

SCHECHTER, M.M. ,GUTIERREZ-MAHONEY, C.G.

The evolution of vertebral angiography.

Neuroradiology, 5 (1973), 157-164.

SCHWARTZ, C.J., MITCHELL, J.R.A.

Atheroma of carotid and vertebral arterial systems.

British Medical Journal, II (1961), 1057-1063.

SELECKI, B.R.

The effects of rotation of the atlas on the axis: experimental work.

Medical Journal of Australia, I (1969), 1012-1015. 
SHANBROM, E., LEVY, L.

The role of systemic blood pressure in cerebral circulation in carotid and basilar artery thromboses. Clinical observations and therapeutic implications of vasopressor agents. American Journal of Medicine, 23 (1957), 197-204.

SHEEHAN, S., BAUER, R.B., MEYER, J.S.

Vertebral artery compression in cervical spondylosis. Arteriographic demonstration during life of vertebral artery insufficiency due to rotation and extension of the neck. Neurology, $10(1960)$, 968-986.

SHERMAN, D.G., HART, R.G., EASTON, J.D.

Abrupt change in head position and cerebral infarction.

Stroke, $12(1981), 2-6$.

SINDERMANN, F.

Krankheidsbild und Kollateralkreislauf bei einseitigem und doppelseitigem Carotisverschluss.

Journal of the Neurological Sciences, 5 (1967), 9-25.

SMITH, D.R., VANDEARK, G.D., KEMPE, L.G.

Cervical spondylosis causing vertebrobasilar insufficiency: a surgical treatment.

Journal of Neurology, Neurosurgery and Psychiatry, 34 (1971), 388-392.

SPENCER, M.P.

An overview of non-invasive cerebrovascular evaluation using Doppler ultrasound.

In: Cerebrovascular evaluation with Doppler ultrasound. ed. Spencer, M.P., Reid, J.M., Martinus Nijhoff, The Hague, (1981), 1-22.

SPENCER, M.P., REID, J.M., BROCKENBROUGH, E.C., THOMAS. G.I.

Non-invasive cerebrovascular evaluation.

Institute of Applied Physiology and Medicine, Seattle, (1977).

SPENCER, M.P., ARTS, TH.

Hemodynamic principles for cardiac Doppler diagnosis.

In: Cardiac Doppler diagnosis, Martinus Nijhoff Publishers, Dordrecht, (1984), 131141.

STEHBENS, W.E.

Turbulence of blood flow.

Quarterly Journal of experimental Physiology, 44 (1959), 110-116.

STEIN, B.M., MCCORMICK, W.F., RODRIGUEZ, J.M., TAVERAS, J.M.

Postmortem angiography of cerebral vascular system.

Archives of Neurology, 7 (1962), 545-559.

STROTHER, C.M., SACKETT, J.F., CRUMMY, A.B., LILLEAS, F.G., ZWIEBEL, W.J., TURNISPEED, W.D., JAVID, M., MISTRETTA, C.A., KRUGER, R.A., ERGUN, D.L., SHAW, C.G.

Clinical applications of computerized fluroscopy. The extracranial carotid arteries.

Radiology, $136(1980), 781-783$. 
SUSSEE, H.J.

Zur Prophylaxe der Zervikalchondrose.

Medizinische Welt, (1955), 673-675.

TAKAHASHI, M., WILSON, G., HANAFEE, W.

Catheter vertebral angiography: a rewiew of 300 examinations.

Journal of Neurosurgery, 30 (1969), 722-731.

TATLOW, W.F.T., BAMMER, H.G.

Syndrome of vertebrall artery compression.

Neurology, 7 (1957), 331-340.

TAYLOR, A.R.

Mechanism and treatment of spinal-cord disorders associated with cervical spondylosils.

Lancet, 264 (1953), 717-720.

TEPE, H.J.

Die Häufigkeit osteochondrotischer Röntgenbefunde der Halswirbelsäule bei 40 symptomfreien Erwachsenen.

Fortschritte auf dem Gebiete der Röntgenstrahlen und der Nuklearmedizin, 85 (1956), 659-663.

THEVENET, A.

Chirurgie de l"artère, vertébrale.

In: Encyclopédie Médico-Chirurgịcale (Paris). S.G.I.M., Les Martres-de-Veyre, No. $76800,43175,3.21 .02,(1979)$.

THIELE, B.L., STRANDNESS, D.E.JR.

Accuracy of angiographic quantification of peripheral atherosclerosis.

Progress in Cardiovascular Diseases, 26 (1983), 223-236.

THOMPSON, J.R., SIMMONS, C.R., HASSO, A.N., HINSHAW, D.B.

Occlusion of the intradural vertebrobasilar artery.

Neuroradiology, 14 (1978), 219-229.

TOOLE, J F.

Cerebrovascular disorders, Raven Press, New York, (1984).

TROOST, B. T.

Dizziness and vertigo in vertebrobasilar disease.

Part 1: Peripheral and systemic causes of dizziness, Stroke, 11 (1980), 301-303.

Part 2: Central canses and vertebrobasilar disease, Stroke, 11 (1980), 413-415.

UEDA, K., TOOLE, J.F., McHENRY, L.C.

Carotid and vertebrobasilar transient ischemic attacks: clinical and angiographic correlation.

Neurology, 29(1979), 1094-1101.

UEDA, K., HOWARD, G., TOOLE, I.F.

Transient ischemic attacks (TIAS) and cerebral infarction (CI): A comparison of predisposing factors.

Journal of Chronic Diseases, 33 (1980), 13-19. 
VIRTAMO, P., KIVALO, E.

Impressions of the vertebral artery by deformations of the unco-vertebral joints.

Acta Radiologica, 48 (1957), 410-412.

VOLLMAR, J. von, EL BAYAR, M., KOLMAR, D., PFLEIDERER, TH., DIE. ZEL, P.B.

Zerebrale Durchblutungsinsuffizienz bei Verschlussprozessen der Arteria subclavia ('subclavian steal effect").

Deutsche Medizinische Wochenschrift, 90 (1965), 8-14.

VRIES, A.R. de

Digitale subtractie angiografie van de carotisbifurcatie.

Thesis, Elinkwijk, B.V., Utrecht, (1984).

WALKER, D.W., ACKER, J.D., COLE, C.A.

Subclavian steal syndrome detected with duplex pulsed Doppler sonography.

American Journal of Neuroradiology, 3 (1982), 615-618.

WHISHART, D.L.

Complications in vertebral angiography as compared to non-vertebral cerebral angiography in 441 studies.

American Journal of Roentgenology, $113(1971), 527-537$.

WHITE, D.N., CURRY G.R., STEVENSON, R.J.

Recording vertebral artery blood flow.

In: Ultrasound in Medicine 4, ed. White, D.N. "Lyons, E.A., Plenum Press, New York, (1978).

WHITE, D.N., KETELAARS, E.J., CLEDGET'T, P.R.

Non-invasive techniques for the recording of vertebral artery flow and their limitations.

Ultrasound in Medicine and Biology, 6 (1980), 315-327.

WILLIAMS, D. , WILSON, T.G.

The diagnosis of the major and minor syndromes of basilar insufficiency.

Brain, 85 (1962), 741-774.

WOOD, C.P.L., MEIRE, H.B.

A technique for imaging the vertebral artery using pulsed Doppler ultrasound.

Ultrasound in Medicine and Biology, 6 (1980), 329-339.

WOOD, C.P.L., MEIRE, H.B.

A new technique for imaging the vertebral artery using pulsed. Doppler ultrasound, In: Blood flow, theory and practice, ed. Taylor, D.E.M., Stevens, A.L., Academic Press, London, (1983), 207-234.

WOOD, G.W., LUKIN, R.R., TOMSICK, TH.A., CHAMBERS, A.A.

Digital subtraction angiography with intravenous injection. Assessment of $\downarrow, 000 \mathrm{ca-}$ rotid bifurcations.

American Journal of Roentgenology, $140(1983), 855-859$. 
WRIGHT, N.L.

Dissection study and measuration of the human aortic arch.

Journal of Anatomy, 104 (1969), 377-385.

WYLIE, E.J, EHRENFELD, W.K.

Extracranial occlusive cerebrovascular disease. Diagnosis and management.

W.B. Sanders Company, Philadelphia, (1970).

YATES, P. O. HUTCHINSON, E.C.

Cerebral infarction: The role of the stenosis of the extra-cranial cerebral arteries.

Medical Research Council special report series, no. 300, Her Majesty's Stationery Office, Londen, (1961).

ZARINS, C.K., GIDDENS, D.P., BHARADVAJ, B.K., SOTTIURAI, V.S., MABON, R.F., GLAGOV, S.

Carotid bifurcation atherosclerosis. Quantitative correlation of plaque localization with fllow velocity profilles and wall sheer stress.

Circulation Research, 53 (1983), 502-514.

ZBORNIKOVA, V., AKESSON, J.A. LASSVIK, C.

Diagnosis of carotid artery disease - comparison between directional Doppler, duplex scanner and angiography.

Acta Neurologica Scandinavia, 65 (1982), 335-346.

ZIEGLER, D.K, HASSANEIN, R.S.

Prognosis in patients with transient ischemic attacks.

Stroke, 4 (1973), 666-673.

ZIR, L.M., MILLER, S.W., DINSMORE, R.E., GILBERT, J.P., HARTHORNE, J.W.

Interobserver variability in coronary angiography.

Circulation, 53 (1976), 627-632. 


\section{ACKNOWLEDGEMENTS}

This study would never have been finished without the support of many people.

In the first place I would like to express my gratitude to prof. J.M.F.A. Mol and prof. R.S. Reneman, for their stimulating ideas and critical review necessary for completing the work compilled in this book.

I would also like to acknowledge the special support of prof. D.E. Strandness of the Department of Surgery of the University of Washington, Seattle, USA, who was so kind to send two of his technicians to our laboratory to teach us the ropes of ultrasonic duplex scanning. Initially, our rapid improvement was to a large extent due to the experience and knowledge of these American sonographers, Jean Primozich and Ramona Lawrence.

Special thanks must also be reserved for the technicians Hannie SchipperSlowikowski, Marian van de Windt, Jaap van Doorn and in particular Hans Hoeneveld. Their excellent work in the duplex laboratory has been the basis for the results described in this study.

I owe thanks to Arnold Hoeks and Paul Hick for their readiness to explore the sampling function of our pulsed Doppler transducers.

I would like to thank Dirk Schipper for his high quality photographs and Saskia Schuurman for the artistic cover design and my mother for her advices in preparing the lay out.

I feel much obliged to Judy Hammacher-Pitt for her scrupulous corrections of the manuscripts and especially to Hanneke de Wal-te Riele who did all the secreterial work and typewriting.

The last chapter was translated into French by F.E.E. Vermeulen, in German by H.J. During, in Japanese by J. Bongenaar and in Indonesian by C. Tirtaman.

Last but not least I would like to thank my family. Neeltje it was your support that helped me much more in my moments of stress than you have realized. Mascha, Merlijn and Jochum one of the most difficult problems was to explain to you the motives of writing this thesis. I really hope that in the future you will be able to understand and to forgive any lapse I might have had in fulfilling my duty as a father. 


\section{CURRICULUM VITAE}

Robert G.A. Ackerstaff was born on the 2nd of April 1942 in Surabaja, Indonesia. After the Second World War his parents moved to The Netherlands were he completed grammar school in Eindhoven. From 1962 he studied at the Medical Faculty of the University of Utrecht, The Netherlands, and graduated in 1969. In 1970 he started his neurological education at the Departments of Neurology, Neurosurgery, Psychiatry and Clinical Neurophysiology of the University Hospital (Heads at the time: Prof. Dr. A. Kemp, Prof. Dr. H. Verbiest, Prof. Dr. J.H. Plokker and Prof. Dr. W. Storm van Leeuwen, respectively) and at the department of Clinical Neurophysiology of the St. Antonius Hospital (Head at the time: Dr. A.J.R. Simons) in Utrecht. In April 1975 he graduated as a neurologist and started working as full-time clinical neurophysiologist in the St. Antonius Hospital. He has been chairman of the Department of Clinical Neurophysiology of the St. Antonius Hospital, Nieuwegein, The Netherlands from January 1984. 